\section{NASA \\ Reference \\ Publication \\ 1102}

\section{NBSIR \\ 82-1672}

April 1983

\section{Transport \\ Properties \\ of Oxygen}

H. M. Roder
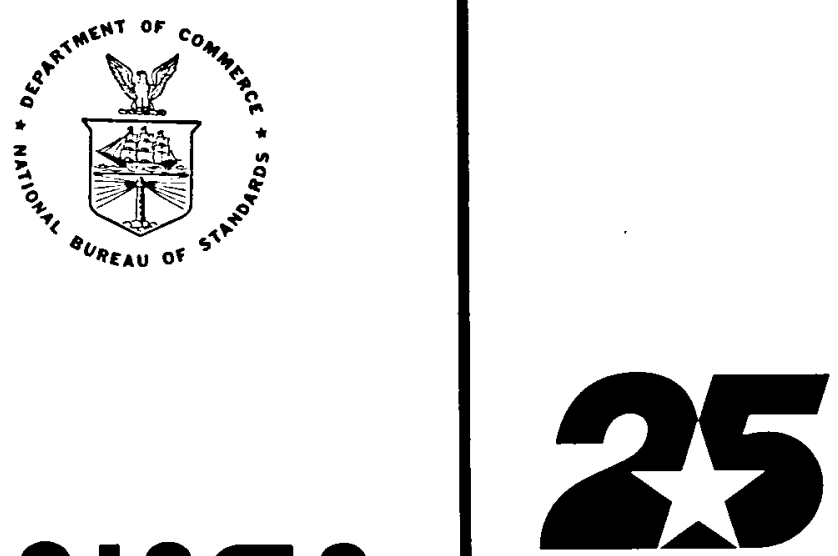

NASA

Reference

Publication

1102

NBSIR

82-1672

1983

\section{Transport \\ Properties \\ of Oxygen}

H. M. Roder

Thermophysical Properties Division

National Engineering Laboratory

National Bureau of Standards

Boulder, Colorado

Prepared for NASA Lewis Research Center 

Contents

Page

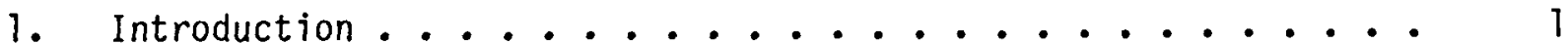

2. Representation of the Viscosity .......... 3

3. Representation of the Thermal Conductivity ........ 4

3.1 Calculations for Region 1 ............ 7

3.2 Calculations for Region 2 ................. 8

3.3 Boundaries for Regions 1 and $2 \ldots \ldots 9$

4. Representation of the Thermal Diffusivity ........ 9

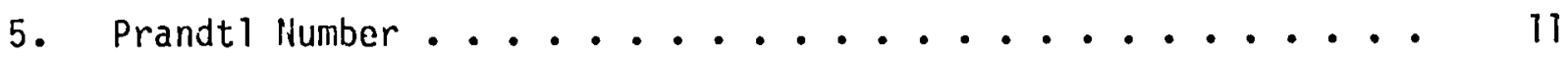

6. Description of the Data Tables ............... 13

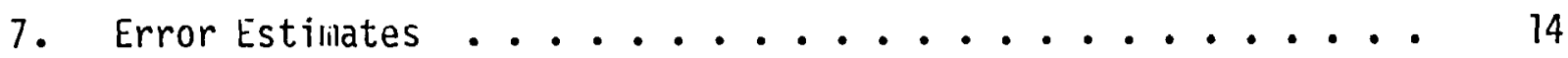

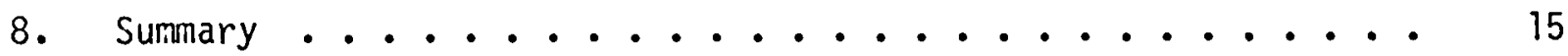

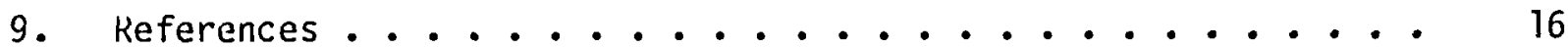

Appendix A. Program Listings for Viscosity ......... 80

Appendix B. Program Listings for Thermal Conductivity . . . . . 81

Appendix C. Conversion Factors, 0xygen .......... 83

\section{List of Figures}

Figure 1. Region of the PVT surface covered by the new thermal conductivity measurements $[8] \ldots \ldots$

List of Tables

Table 1. Overview of Previous Publications .......... 2

Table 2. Thermal Conductivities near $162.9805 \mathrm{~K} \ldots \ldots$

Table 3. Thermal Conductivities from Lightscattering, Weber [9] • . 12

Table 4. Transport Properties of Oxygen for saturated Liquid and Vapor, SI Units ................. 17

Table 5. Transport Properties of 0xygen, Isobars, SI Units . . . 18

Table 6. Transport properties of Oxygen for saturated Liquid and Vapor, Engr. Units ............ 50

Table 7. Transport Properties of Oxygen, Isobars, Engr. Units ... 52 



\title{
TRANSPORT PROPERTIES OF OXYGEN
}

\author{
H. M. Roder \\ Thermophysical Properties Division \\ National Engineering Laboratory \\ National Bureau of Standards \\ Boulder, Colorado 80303
}

\section{Introduction}

An accurate thermophysical properties base for fluid oxygen has been developed through a series of measurements and analyses. Table 1 presents a chronology of prior publications which contain equations of state, correlations of the transport properties, data tables for the transport properties, or a combination of these items. Table 1 shows that the massive set of tables for oxygen in NASA SP 3071 [2] has gradually been extended and superseded by new measurements and new correlations. Specifically there have been new experimental measurements of viscosity [4], a new correlation of viscosity and thermal conductivity [3], an extension of the PVT measurements from $34 \mathrm{MPa}(5000 \mathrm{psia})$ to $80 \mathrm{MPa}$ (12000 psia) [5], a new equation of state which includes the extension in pressure [6], the development of an interactive package for the equation of state [7], new experimental thermal conductivity measurements [8], and new experimental thermal diffusivity measurements near the critical point [9]. The present tables were computed because previous tables of the transport properties of oxygen extend to only $34 \mathrm{MPa}$ (5000 psia), and because for thermal conductivity the new measurements $[8,9]$ show the previously best correlation $[3]$ to be in error by up to 35 percent for nearly all temperatures at the highest pressures. 
Table 1. Overview of Previous Publications

\begin{tabular}{|c|c|c|c|c|c|c|}
\hline Reference & Year & \multicolumn{2}{|c|}{ PVT } & Transport & Properties & Notes \\
\hline & & Equation & Data Tables & Equations & Data Tables & \\
\hline [1] NBS Tech. Note 384 & 1971 & polynomial & isobars to 5000 psia & yes & yes & temperature range triple point to $340 \mathrm{~K}$ \\
\hline 12] NASA SP 3071 & 1972 & polynomial & $\begin{array}{l}\text { isobars to } 340 \mathrm{~atm} \\
\text { and } 5000 \mathrm{psia}\end{array}$ & ref. 1 & yes & \\
\hline $\begin{array}{l}\text { [3] J. Phys. Chem. Ref. } \\
\text { Data }\end{array}$ & 1974 & mod. BWR & no & new & yes & $\begin{array}{l}\text { new correlation of transport properties } \\
\text { uses the data of }|4|\end{array}$ \\
\hline [4] Physica & 1977 & ref. 3 & no & ref. 3 & no & new viscosity measurements \\
\hline [5] NASA RP 1011 & 1977 & polynomial & $\begin{array}{l}\text { Isobars to } 1000 \text { bar } \\
\text { and } 15000 \text { psia }\end{array}$ & no & no & incorporates new PVT data to 800 bar \\
\hline [6] NBS IR 78-882 & 1978 & mod. BWR & no & ref. 3 & no & $\begin{array}{l}\text { Incorporates revised transport } \\
\text { property correlations. }\end{array}$ \\
\hline (7) NBS Tech. Note 1025 & 1980 & mod. BWR & no & ref. 3 & no & Incorporates BWR into "Fluids Pack" \\
\hline [8] J. Res. NBS (in press) & 1982 & ref. 7 & no & new & no & new thermal conductivity measurements \\
\hline $\begin{array}{l}\text { [9] Int. J. Thermophysics } \\
\text { (in press) }\end{array}$ & 1982 & $\begin{array}{l}\text { poly. and } \\
\text { mod. BWR }\end{array}$ & no & no & no & new thermal diffusivity measurements \\
\hline present report & 1982 & mod. BWR & $\begin{array}{l}\text { Isobars to } 100 \mathrm{MPa} \\
\text { and } 15000 \mathrm{psia}\end{array}$ & $\begin{array}{l}\text { ref. } 3 \\
\text { and new }\end{array}$ & yes & incorporates new thermal conductivity data \\
\hline
\end{tabular}




\section{Representation of the Viscosity}

Values of the viscosity are calculated from the correlation of Hanley, et al. [3]. For the correlation the authors surveyed the literature and systematically selected data which met particular criteria for accuracy. For oxygen the experimental measurements of Haynes [4] with an accuracy of 2 percent were selected. This set of data was measured with a torsionally oscillating quartz crystal, and it comprises some 200 points at temperatures from 75 to $300 \mathrm{~K}$ with pressures to $34 \mathrm{MPa}$. The correlation expresses viscosity in terins of density and temperature, and it requires an equation of state (EOS) to translate pressures into equivalent densities. The EOS most commonly used is the interactive package by liccarty [7]. The dependence of viscosity on temperature and density is expressed in [3] as

$$
\eta(\rho, T)=\eta_{0}(T)+\eta_{T}(T) \rho+\Delta \eta(\rho, T)+\Delta \eta_{C}(\rho, T),
$$

where

$$
\begin{aligned}
\eta_{0}=g_{7} T^{-1} & +g_{2} T^{-2 / 3}+g_{3} T^{-1 / 3}+g_{4}+g_{5} T^{1 / 3}+g_{6} T^{2 / 3}+g_{7} T \\
& +g_{8} T^{4 / 3}+g_{9} T^{5 / 3},
\end{aligned}
$$

and

$$
\eta_{1}(T)=A+B[C-\ln (T / F)]^{2},
$$

and

$$
\begin{aligned}
\Delta n(\rho, T) & =D \exp \left[k_{1}+k_{2} / T\right]\left\{\operatorname { e x p } \left[\rho^{0.1}\left(k_{3}+k_{4} / T^{3 / 2}\right)\right.\right. \\
& \left.\left.+\theta_{\rho}^{0.5}\left(k_{5}+k_{6} / T+k_{7} / T^{2}\right)\right]-1.0\right\},
\end{aligned}
$$

where $\theta$ is a density dependent factor given by $\theta=\left(p-\rho_{c}\right) / \rho_{c}$.

While there is evidence for an enhancement of viscosity near the critical point, teril 4 in eq $(1), \Delta \eta_{c}(\rho, T)$, is not considered in reference [3]. * Proyram listings to calculate the viscosity [7] are given in appendix A. For completeness the values of the coefficients for eqs (2-4) are listed below. For these

\footnotetext{
${ }^{\star}$ A full description of how to calculate $\Delta \eta_{C}(\rho, T)$ is given in reference [10].
} 
coefficients the units implied are temperature in $\mathrm{K}$, density in $\mathrm{g} / \mathrm{cm}^{3}$, and viscosity in $\mu \mathrm{g} / \mathrm{cm} \bullet \mathrm{s}$.

Equation (2)

$\begin{array}{ll}g_{1}=-9.7076378593 \mathrm{E}+1 & \mathrm{~A}=1.352652 \\ g_{2}=8.2801254201 \mathrm{E}+1 & B=-2.036126 \\ g_{3}=-2.4668758803 \mathrm{E}+1 & \mathrm{C}=1.4 \\ g_{4}=2.1324360243 & \mathrm{~F}=100.0 \\ g_{5}=3.7851049522 \mathrm{E}-1 & \\ g_{6}=-1.0487216090 \mathrm{E}-1 & \\ g_{7}=1.1134441304 \mathrm{E}-2 & \\ g_{8}=-5.3676093757 \mathrm{E}-4 & \\ g_{9}=1.0279379641 \mathrm{E}-5 & \end{array}$

Equation (3)

$A=4.352652$

$D=1.0 \mu \mathrm{g} / \mathrm{cm} \mathrm{s}$

$k_{1}=-12.15239$

$k_{2}=2.434069 \times 10^{2}$

$k_{3}=18.20116$

$k_{4}=-2.749896 \times 10^{3}$

$k_{5}=-0.3142173$

$k_{6}=1.191150 \times 10^{2}$

$k_{7}=2.739043 \times 10^{2}$

$\rho_{c}=0.435 \mathrm{~g} / \mathrm{cm}^{3}$

3. Representation of the Thermal Conductivity

Values of the thermal conductivity are calculated from the correlation of Roder [8]. This new correlation is based on new experimental measurements of the thermal conductivity $[8 \mathrm{j}$ and new experimental measurements of the thermal diffusivity [9]. The new conductivity values [8] comprise 1126 points measured on 13 isotherms from 78 to $310 \mathrm{~K}$ with pressures to $70 \mathrm{MPa}$. The measurements were made with a new transient hot wire apparatus.

The new correlation expresses thermal conductivity as a function of density and temperature rather than temperature and pressure because over a wide range of experimental conditions the behavior of thermal conductivity is dominated by its density dependence. The technique requires an equation of state [7] to translate measured pressures into equivalent densities. The dependence of thermal conductivity on temperature and density is normally expressed as

$$
\lambda(\rho, T)=\lambda_{0}(T)+\lambda_{\text {excess }}(\rho, T)+\Delta \lambda_{\text {critical }}(\rho, T)
$$

The first term on the right of eq (1) is the dilute gas term which is independent of density. The second is the excess thermal conductivity. The first two terms taken together are sometimes called the "background" thermal conductivity. The final term is the critical point enhancement. The dilute gas term is expressed as 


$$
\begin{aligned}
\lambda_{0}(T)=\left[A_{1} T^{-1}\right. & +A_{2} T^{-2 / 3}+A_{3} T^{-1 / 3}+A_{4}+A_{5} T^{T / 3}+A_{6} T^{2 / 3} \\
& \left.+A_{7} T+A_{8} T^{4 / 3}+A_{9} T^{5 / 3}\right] / 1000
\end{aligned}
$$

with $\lambda_{0}$ in $W / m \cdot K$ and $T$ in kelvin.

The expression used for the excess thermal conductivity is as follows:

$$
\lambda_{\text {excess }}(\rho, T)=\alpha \rho+\delta\left[e^{\beta \rho^{\gamma}}-1.0\right]
$$

where the parameters $\alpha, \beta, \gamma, \delta$ are functions of temperature as follows:

$$
\begin{array}{ll}
\alpha=B_{7} T & \gamma=B_{5}+B_{6} T+B_{7} T^{2} \\
\beta=B_{2}+B_{3} T+B_{4} T^{2} & \delta=B_{8}+B_{9} T+B_{10} / T^{2} .
\end{array}
$$

The coefficients for eqs (6) and (7) with $T$ in kelvins and $\lambda$ in $W / m \circ K$ are Equation (6)

$$
\begin{aligned}
& A_{1}=-2.0395052193 \mathrm{E}+5 \\
& A_{2}=2.4088141709 \mathrm{E}+5 \\
& A_{3}=-1.2014175183 \mathrm{E}+5 \\
& A_{4}=3.295494919 \mathrm{E}+4 \\
& A_{5}=-5.4244239598 \mathrm{E}+3 \\
& A_{6}=5.4734865540 \mathrm{E}+2 \\
& A_{7}=-3.2854821539 \mathrm{E}+1 \\
& A_{8}=1.0753572103 \\
& A_{9}=-1.4610986820 \mathrm{E}-2
\end{aligned}
$$

Equation (7)

$$
\begin{aligned}
& B_{1}=.298644 E-5 \\
& B_{2}=.59842 E+00 \\
& B_{3}=.11362 E-01 \\
& B_{4}=-.19520 E-04 \\
& B_{5}=.47624 E+00 \\
& B_{6}=-.64769 E-03 \\
& B_{7}=.83223 E-06 \\
& B_{8}=-.278141 E-4 \\
& B_{9}=.153705 E-6 \\
& B_{10}=.147176 E+1
\end{aligned}
$$

The calculation of the third term in eq (5) is split into two separate regions which are shown in figure 1 . The first region is called the critical region proper, and it corresponds roughily to the range of conditions for which Sengers, et a1. [10] recommend the use of a scaled equation of state. The second region is called the extended critical region. It is shown as a triangle in figure 1 


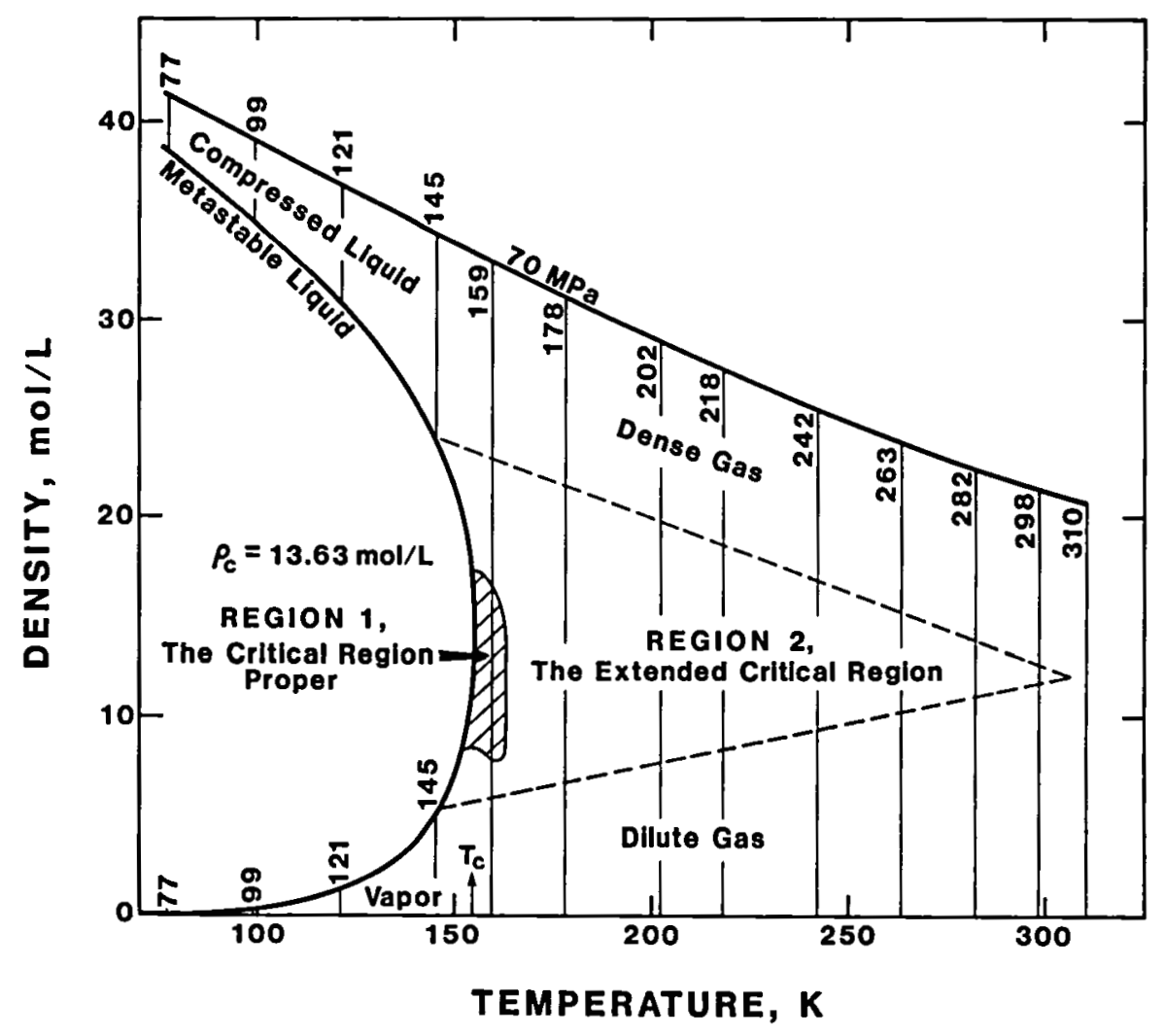

Figure 1. Region of the PVT surface covered by the new thermal conductivity measurements [8]. Also shown are regions 1 and 2 for the calculation of the critical point enhancement. The new thermal diffusivity measurements [9] are nearly all located in region 1. 
and covers those densities and temperatures for which the new measurements reveal an anomalous increase above the background conductivity.

3.1. Calculations for Region 1

We define the reduced variables $T^{*}=T / T_{C}, \rho^{*}=\rho / \rho_{C}, P^{\star}=P / P_{C}$ and

$$
\Delta T^{*}=\left(T-T_{C}\right) / T_{C} \text { and } \Delta \rho^{*}=\left(\rho-\rho_{C}\right) / \rho_{C}
$$

The scaling variables are defined by

$$
x=\Delta T^{*} /\left|\Delta \rho{ }^{*}\right|^{T / \beta} \text { and } y=\frac{x+x_{0}}{x_{0}}
$$

The symmetrized compressibility is defined by

$$
X_{T}^{*}=\left|\Delta \rho^{*}\right|-\gamma / \beta\left[\frac{\left[(1+E) /\left(1+E y^{2 \beta}\right)\right]^{(\gamma-1) / 2 \beta}}{D\left[\delta+(y-1)\left(\delta-\beta^{-1}+E y^{2 \beta}\right) /\left(1+E y^{2 \beta}\right)\right]}\right]
$$

for which a special case occurs at the critical isochore where $\Delta p^{*}=0$

$$
x_{T}^{*}=\Gamma\left|\Delta T^{*}\right|^{-\gamma} \text {. }
$$

The coefficient $\Gamma$ in eq (12) is defined by

$$
\Gamma=\frac{x_{0}^{\gamma}}{D}\left(\frac{1+E}{E}\right)^{(\gamma-1) / 2 \beta} .
$$

Given the definitions above, Sengers, et al. [10] estimate the critical enhancement from

$$
\begin{aligned}
\Delta \lambda_{\text {critical }}(\rho, T) & =\frac{\Lambda}{\Xi_{0}} \frac{k_{B} P}{6 \pi}\left(\frac{T^{*}}{\rho^{\star}}\right)^{2}\left(\frac{\partial P^{*}}{\partial T^{\star}}\right)_{\rho}^{2} \chi_{T}^{*}(\gamma-\nu) / \gamma_{\rho}^{* n} \exp \left[-\left\{A_{\lambda}\left(\Delta T^{\star}\right)^{2}\right.\right. \\
& \left.\left.+B_{\lambda}\left(\Delta \rho^{\star}\right)^{4}\right\}\right] .
\end{aligned}
$$

This equation relates the thermal conductivity enhancement at a given temperature and density to the pressure coefficient $(\partial P / \partial T)_{\rho}$, the symmetrized compressibility $x_{T}$ and the (normal) shear viscosity $n$. 
The values to be used with eqs (9-14) for oxygen are

$$
\begin{aligned}
& T_{c}=154.581 \mathrm{~K} \\
& \mathrm{n}=0 \\
& \Lambda=1.04 \\
& A_{\lambda}=39.8 \\
& B_{\lambda}=5.45 \\
& \Xi=5.9783 \times 10^{-10} \\
& B=0.355 \\
& \gamma=1.190 \\
& P_{C}=49.77054 \mathrm{~atm} \\
& k_{B}=1.38054 * 10^{-23} \mathrm{~J} / \mathrm{K} \\
& \delta=4.352 \\
& D=2.36 \\
& E=0.287 \\
& x_{0}=0.183
\end{aligned}
$$

The prescription given by Sengers, et at. [10] has been modified in two minor ways. First, the value of $\Lambda$ adopted to be 1.02 for $\mathrm{CO}_{2}$ in [10] is chosen to be 1.04 for oxygen. This number is established as a best value for Weber's new experimental diffusivity measurements [9], see section 4. The second modification is to extend the calculation using the scaled equation out to a temperature of $162.9805 \mathrm{~K}$ or $1.054 \mathrm{~T}_{\mathrm{c}}$ rather than $1.03 \mathrm{~T}_{\mathrm{c}}$.

\subsection{Calculations for Region 2}

The expressions used in this region are as follows [8].

$$
\Delta \lambda_{\text {critical }}(\rho, T)=\left\{c_{1} /\left(T+c_{2}\right)+c_{3}+c_{4} T\right\} \cdot e^{-x^{2}}
$$

we define

$$
\rho_{\text {center }}=\rho_{c}+C_{5}\left(T-T_{c}\right)^{1.5} \text {, }
$$

then the variable $x$ in eq (15) is

$$
x=C_{6}\left(\rho-\rho_{\text {center }}\right) \quad \text { for } \rho>\rho_{\text {center }} \text {, }
$$

and

$$
x=c_{6}\left(\rho-\rho_{\text {center }}\right)+c_{7}\left(\rho-\rho_{\text {center }}\right)^{5} \text { for } \rho<\rho_{\text {center }} \text {. }
$$

The coefficients for eqs (15-18) are

$$
\begin{array}{lc}
T_{c}= & 154.581 \mathrm{~K} \\
\rho_{c}= & 13.63 \mathrm{~mol} / \mathrm{L} \\
C_{1}= & .219200 \mathrm{E}+0 \\
C_{2}= & -145.55 \\
C_{3}= & .734512 \mathrm{E}-02
\end{array}
$$$$
\begin{aligned}
& C_{4}=-.282950 E-04 \\
& C_{5}=-.71599 E-3 \\
& C_{6}=.13804 E+0 \\
& C_{7}=.12980 E-5
\end{aligned}
$$ 


\subsection{Boundaries for Regions 1 and 2}

The four boundaries defining the two different regions of computation are the saturated liquid and vapor at the lower temperature, $162.9805 \mathrm{~K}$ at the upper temperature, densities between 8.5 and $7.5 \mathrm{~mol} / \mathrm{L}$ at the lower density, and densities between 18 and $13.6 \mathrm{~mol} / \mathrm{L}$ at the upper density. The boundaries are shown in $\rho-T$ coordinates in figure 1 . For any given temperature between saturation and $162.9805 \mathrm{~K}$ the switch between regions 1 and 2 is accomplished without an abrupt change in the value of $\lambda$. This is done by switching at those densities where the calculated values of thermal conductivity from [8] and [10] are equal.

The switch in calculation at the upper temperature boundary of $162.9805 \mathrm{~K}$ can not, unfortunately, be accomplished without an abrupt change in the value of thermal conductivity. The reason for this is because in reference [10] the centering density is $\rho_{c}$ by definition, whereas in reference [8] the centering density is less than $\rho_{c}$ for all temperatures other than $T_{c}$. The maximum "glitch" along the $162.9805 \mathrm{~K}$ boundary is about 1.6 percent in the value of $\lambda$. The local change across this boundary is shown for densities from 0 to $30 \mathrm{~mol} / \mathrm{L}$ in table 2 .

Programs to calculate the thermal conductivity are new. Listings are given in appendix $B$. The changes required in the program set of reference [7] to implement the new thermal conductivity calculation are indicated in appendix $B$ by arrows.

\section{Representation of the Thermal Diffusivity}

The thermal diffusivity is defined by

$$
\alpha \equiv \lambda / \rho C_{p}
$$

where $C_{p}$ is the heat capacity at constant pressure. Both $\rho$ and $C_{p}$ are normally obtained from an equation of state and the thermal diffusivity is then calculated from the thermal conductivity. Occasionally the thermal diffusivity is measured directly, as was done by Weber [9] who used a light scattering apparatus to measure the thermal diffusivity, primarily in the critical region.

The experimental information to be considered are the 76 points presented in three groups in table 3, where the values are taken from reference [9]. Weber converted his experimental measurements of thermal diffusivity to values of 
Table 2. Thermal conductivities near $162.9805 \mathrm{~K}$

\begin{tabular}{|c|c|c|c|c|c|c|}
\hline $\begin{array}{c}\text { Temoer } \\
\text { Inslde } \\
K\end{array}$ & $\begin{array}{l}\text { atures } \\
\text { outside } \\
k\end{array}$ & $\begin{array}{l}\text { Density } \\
\text { mold }\end{array}$ & $\begin{array}{c}\text { Thermal Cone } \\
\text { Inside } \\
w / m, k\end{array}$ & $\begin{array}{l}\text { sctlyities } \\
\text { outside } \\
w / m, k\end{array}$ & $\begin{array}{l}\text { Devl. } \\
\text { In.-out. } \\
\text { wimok }\end{array}$ & percent \\
\hline $\begin{array}{l}162.990 \\
162.980 \\
162.980 \\
162.980 \\
162.980 \\
162.990 \\
162.990 \\
162.980 \\
162.980 \\
162.980 \\
162.980 \\
162.980 \\
162.980 \\
162.980 \\
162.980 \\
162.980 \\
162.990 \\
162.980 \\
162.980 \\
162.980 \\
162.980 \\
162.990 \\
162.980 \\
162.990 \\
162.980 \\
162.980 \\
162.980 \\
162.980 \\
162.980 \\
162.980 \\
162.990 \\
162.980 \\
162.980 \\
162.980 \\
162.980 \\
162.980 \\
162.980 \\
162.990 \\
162.980 \\
162.980 \\
162.980 \\
162.980 \\
162.990 \\
162.980 \\
162.980 \\
162.980 \\
162.980 \\
162.990 \\
162.980 \\
162.980 \\
162.980 \\
162.980 \\
162.980 \\
162.980 \\
162.980 \\
162.980 \\
162.980 \\
162.980 \\
162.980 \\
162.980\end{array}$ & $\begin{array}{l}162.981 \\
162.981 \\
162.981 \\
162.981 \\
162.981 \\
162.581 \\
162.981 \\
162.991 \\
162.981 \\
162.981 \\
162.981 \\
162.981 \\
162.981 \\
162.981 \\
162.981 \\
162.981 \\
162.981 \\
162.981 \\
162.981 \\
162.981 \\
162.981 \\
162.981 \\
162.981 \\
162.981 \\
162.981 \\
162.981 \\
162.981 \\
162.981 \\
162.981 \\
162.981 \\
162.981 \\
162.981 \\
162.981 \\
162.991 \\
162.981 \\
162.981 \\
162.981 \\
162.981 \\
162.981 \\
162.981 \\
162.981 \\
162.991 \\
162.981 \\
162.981 \\
162.981 \\
162.981 \\
162.981 \\
162.981 \\
162.981 \\
162.981 \\
162.981 \\
162.981 \\
162.981 \\
162.981 \\
162.981 \\
162.981 \\
162.981 \\
162.981 \\
162.981 \\
162.981\end{array}$ & $\begin{array}{l}.5 \\
1.0 \\
1.5 \\
2.0 \\
2.5 \\
3.0 \\
3.5 \\
4.0 \\
4.5 \\
5.0 \\
5.5 \\
6.0 \\
6.5 \\
7.0 \\
7.5 \\
8.0 \\
8.5 \\
9.0 \\
9.5 \\
10.0 \\
10.5 \\
11.0 \\
11.5 \\
12.0 \\
12.5 \\
13.0 \\
13.5 \\
14.0 \\
14.5 \\
15.0 \\
15.5 \\
16.0 \\
16.5 \\
17.0 \\
17.5 \\
18.0 \\
18.5 \\
19.0 \\
19.5 \\
20.0 \\
20.5 \\
21.0 \\
21.5 \\
22.0 \\
22.5 \\
23.0 \\
23.5 \\
24.0 \\
24.5 \\
25.0 \\
25.5 \\
26.0 \\
26.5 \\
27.0 \\
27.5 \\
28.0 \\
28.5 \\
29.0 \\
29.5 \\
30.0\end{array}$ & $\begin{array}{l}.015378 \\
.013030 \\
.016539 \\
.017123 \\
.017795 \\
.018564 \\
.019436 \\
.020412 \\
.021491 \\
.022668 \\
.023938 \\
.025294 \\
.026728 \\
.028231 \\
.029792 \\
.031402 \\
.033214 \\
.035034 \\
.036831 \\
.038586 \\
.040280 \\
.041894 \\
.043411 \\
.044813 \\
.046087 \\
.047229 \\
.048258 \\
.049325 \\
.050342 \\
.051274 \\
.052131 \\
.052928 \\
.053682 \\
.054413 \\
.055141 \\
.055890 \\
.056680 \\
.057533 \\
.058468 \\
.059504 \\
.060656 \\
.061937 \\
.063358 \\
.064927 \\
.066651 \\
.068533 \\
.070576 \\
.072780 \\
.075145 \\
.077671 \\
.080354 \\
.083195 \\
.086390 \\
.089339 \\
.002641 \\
.096095 \\
.099700 \\
.103458 \\
.107369 \\
.111436\end{array}$ & $\begin{array}{l}.015579 \\
.016030 \\
.016539 \\
.017123 \\
.017795 \\
.018564 \\
.019436 \\
.020412 \\
.021491 \\
.022668 \\
.023938 \\
.025294 \\
.026728 \\
.028231 \\
.029792 \\
.031401 \\
.033046 \\
.034713 \\
.036386 \\
.038051 \\
.039692 \\
.041292 \\
.042836 \\
.044310 \\
.045703 \\
.047005 \\
.048213 \\
.049324 \\
.050342 \\
.051274 \\
.052131 \\
.052928 \\
.053681 \\
.054612 \\
.055141 \\
.055889 \\
.056680 \\
.057533 \\
.05868 \\
.059504 \\
.060656 \\
.061937 \\
.063358 \\
.064927 \\
.066551 \\
.068533 \\
.070576 \\
.072780 \\
.075145 \\
.077671 \\
.080354 \\
.083195 \\
.086190 \\
.089340 \\
.092641 \\
.096095 \\
.099700 \\
.103458 \\
.107369 \\
.111436\end{array}$ & $\begin{array}{l}-.000000 \\
-000000 \\
-000000 \\
-000000 \\
-000000 \\
-.000000 \\
-000000 \\
-000000 \\
.000000 \\
.000000 \\
.000000 \\
.000000 \\
.000000 \\
.000000 \\
.000000 \\
.000000 \\
.000168 \\
.000321 \\
.000445 \\
.000535 \\
.000588 \\
.000602 \\
.000575 \\
.000503 \\
.000384 \\
.000224 \\
.000045 \\
.000001 \\
.000001 \\
.000001 \\
.000001 \\
.000001 \\
.000001 \\
.000000 \\
.000000 \\
.000000 \\
.000000 \\
.000000 \\
.000000 \\
.000000 \\
.000000 \\
.000000 \\
.000000 \\
.000000 \\
.000000 \\
.000000 \\
-0000000 \\
-0000000 \\
-0000000 \\
-0000000 \\
-00000000 \\
-000000000 \\
-0000000 \\
-0000000 \\
-0000000 \\
-0000000\end{array}$ & $\begin{array}{l}-.00 \\
-.00 \\
-.00 \\
-.00 \\
-.00 \\
-.00 \\
-.00 \\
-.00 \\
.00 \\
.00 \\
.00 \\
.00 \\
.00 \\
.00 \\
.00 \\
.00 \\
.50 \\
.92 \\
1.21 \\
1.39 \\
1.40 \\
1.44 \\
1.32 \\
1.12 \\
.83 \\
.47 \\
.09 \\
.00 \\
.00 \\
.00 \\
.00 \\
.00 \\
.00 \\
.00 \\
.00 \\
.00 \\
.00 \\
.00 \\
.00 \\
.00 \\
.00 \\
.00 \\
.00 \\
.00 \\
.00 \\
.00 \\
-.00 \\
. .00 \\
-.00 \\
-.00 \\
-.00 \\
-.00 \\
-.00 \\
-.00 \\
-.00 \\
-.00 \\
-.00 \\
-.00 \\
-.00 \\
-.00\end{array}$ \\
\hline
\end{tabular}


thermal conductivity using eq (19). Thus the comparisons to be made are "experimental thermal conductivity" to calculated thermal conductivity, where the method of calculation has been described in the previous section. In table 3 the first group, 14 points, includes all of Weber's measurements with temperatures greater than 158. K, i.e., these points represent an overlap of the experimental measurements of references [8] and [9]. The second group, 33 points, includes those measurements for which the experimental densities 1 ie between 13.1 and $13.7 \mathrm{~mol} / \mathrm{L}$, or $\left|\Delta \rho^{*}\right| \leq 0.04$. In essence this group is quite close to critical density. The remaining 29 points, i.e., the last group, are characterized by experimental densities which are considerably different than critical density. Differences between the calculation and Weber's "thermal conductivity" values [9] are given in table 3. The RMS deviation for the first grouping of 14 points is 5.3 percent. Well within the experimental uncertainty estimated by Weber, this average deviation indicates agreement between the diffusivity experiment and the hot wire thermal conductivity measurements. For the second grouping of 33 points we find an RMS deviation of 7.5 percent, again within the expected uncertainty of the experimental measurements. This average deviation indicates agreement between the diffusivity measurements and the most current method of predicting the anomalous thermal conductivity in the critical region for densities close to critical, i.e., for $\Delta \lambda_{c}\left(\rho_{c}, T\right)$. However, the deviation of the third grouping of 29 points is nearly 27 percent RMS, which exceeds experimental uncertainty by a considerable margin, and indicates a basic disagreement between these measurements and the prediction for densities which are substantially different from critical density. Despite considerable effort, the cause of the disagreement remains unresolved. The calculation values off the critical isochore nay, therefore, be uncertain by as much as 30 percent for both thermal diffusivity and thermal conductivity.

\section{Prandtl Number}

The Prandtl number, $\mathrm{Pr}$, is defined by the relation

$$
\operatorname{Pr} \equiv C_{p} n / \lambda
$$

where $C_{p}$ is the specific heat at constant pressure, $n$ the viscosity and $\lambda$ the thermal conductivity. To conserve space the Prandtl number has not been presented in the tables, it is however quite easy to calculate the Prandtl number 
Table 3. Thermal conductivities from liohtscattering, Weber 693.

\begin{tabular}{|c|c|c|c|c|c|c|}
\hline $\begin{array}{c}\text { Temperature } \\
k\end{array}$ & $\begin{array}{c}\text { Density } \\
m 01 / t\end{array}$ & $\begin{array}{c}\text { Thermal } \\
\text { exp. } \\
\text { W/m.K }\end{array}$ & $\begin{array}{c}\text { Conductivity } \\
\text { celc. } \\
\text { w/mok }\end{array}$ & $\begin{array}{c}\text { Deviat } \\
\text { exp.-calce } \\
H / m \in K\end{array}$ & $\begin{array}{l}\text { Dons } \\
\text { percent }\end{array}$ & $\begin{array}{l}\text { Group } \\
\text { RHS } \\
\text { percent }\end{array}$ \\
\hline $\begin{array}{l}158.519 \\
161.847 \\
164.426 \\
164.426 \\
164.426 \\
166.796 \\
168.946 \\
173.935 \\
150.583 \\
159.890 \\
159.883 \\
159.878 \\
158.133 \\
160.493\end{array}$ & $\begin{array}{l}13.19 \\
13.14 \\
13.27 \\
13.27 \\
13.27 \\
13.08 \\
13.21 \\
13.04 \\
13.49 \\
13.44 \\
13.58 \\
13.50 \\
15.30 \\
15.30\end{array}$ & $\begin{array}{l}.0556 \\
.0480 \\
.0482 \\
.0470 \\
.0444 \\
.0415 \\
.0440 \\
.0414 \\
.0541 \\
.0525 \\
.0537 \\
.0527 \\
.0593 \\
.0550\end{array}$ & $\begin{array}{l}.0562 \\
.0490 \\
.0468 \\
.0488 \\
.0468 \\
.0453 \\
.0449 \\
.0433 \\
.0538 \\
.0530 \\
.0533 \\
.0531 \\
.0589 \\
.0542\end{array}$ & $\begin{array}{r}-.0006 \\
-.0010 \\
.0014 \\
.0002 \\
-.0024 \\
-.0038 \\
-.0009 \\
-.0019 \\
.0003 \\
-.0005 \\
.0004 \\
-.0004 \\
.0104 \\
.0008\end{array}$ & $\begin{array}{r}-1.16 \\
-2.08 \\
2.82 \\
-34 \\
-5.50 \\
-9.15 \\
-1.94 \\
-4.49 \\
.57 \\
-.98 \\
.80 \\
-.84 \\
15.03 \\
1.44\end{array}$ & 5,73 \\
\hline $\begin{array}{l}154.619 \\
155.173 \\
155.649 \\
155.651 \\
156.642 \\
157.580 \\
154.717 \\
154.746 \\
154.771 \\
154.787 \\
154.795 \\
154.645 \\
154.690 \\
154.801 \\
154.841 \\
154.872 \\
155.025 \\
155.034 \\
155.271 \\
155.524 \\
155.546 \\
155.769 \\
156.003 \\
156.500 \\
157.000 \\
157.468 \\
154.595 \\
154.598 \\
154.590 \\
154.503 \\
154.517 \\
154.628 \\
154.578\end{array}$ & $\begin{array}{l}13.13 \\
13.18 \\
13.19 \\
13.20 \\
13.19 \\
13.18 \\
13.33 \\
13.37 \\
13.40 \\
13.38 \\
13.39 \\
13.42 \\
13.44 \\
13.44 \\
13.46 \\
13.43 \\
13.47 \\
13.53 \\
13.54 \\
13.54 \\
13.57 \\
13.57 \\
13.58 \\
13.58 \\
13.58 \\
13.58 \\
13.63 \\
13.63 \\
13.63 \\
13.63 \\
13.63 \\
13.63 \\
13.63\end{array}$ & $\begin{array}{l}.2507 \\
.1043 \\
.0875 \\
.0871 \\
.0715 \\
.0627 \\
.1842 \\
.1758 \\
.1674 \\
.1601 \\
.1578 \\
.2610 \\
.2109 \\
.1627 \\
.1567 \\
.1426 \\
.1212 \\
.1230 \\
.1100 \\
.0990 \\
.0970 \\
.0891 \\
.0855 \\
.0783 \\
.0731 \\
.0643 \\
.5600 \\
.5180 \\
.5230 \\
.4720 \\
.3550 \\
.3170 \\
.2250\end{array}$ & $\begin{array}{l}.2855 \\
.0996 \\
.0812 \\
.0812 \\
.0665 \\
.0601 \\
.1835 \\
.1697 \\
.1600 \\
.1543 \\
.1520 \\
.2635 \\
.2063 \\
.1507 \\
.1404 \\
.1337 \\
.1129 \\
.1121 \\
.0956 \\
.0856 \\
.0850 \\
.0793 \\
.0749 \\
.0685 \\
.0643 \\
.0614 \\
.5831 \\
.5266 \\
.5111 \\
.4605 \\
.3578 \\
.3129 \\
.2198\end{array}$ & $\begin{array}{r}-.0348 \\
.0047 \\
.0063 \\
.0059 \\
.0050 \\
.0026 \\
.0007 \\
.0061 \\
.0074 \\
.0058 \\
.0058 \\
-.0025 \\
.0046 \\
.0120 \\
.0163 \\
.0089 \\
.0083 \\
.0109 \\
.0144 \\
.0134 \\
.0120 \\
.0098 \\
.0106 \\
.0098 \\
.0088 \\
.0029 \\
-.0231 \\
-.0086 \\
.0119 \\
.0115 \\
-00028 \\
.0041 \\
.0052\end{array}$ & $\begin{array}{r}-13.90 \\
4.51 \\
7.16 \\
6.74 \\
7.03 \\
4.12 \\
.35 \\
3.47 \\
4.40 \\
3.64 \\
3.68 \\
-.95 \\
2.17 \\
7.36 \\
10.37 \\
6.21 \\
6.88 \\
8.87 \\
13.09 \\
13.52 \\
12.40 \\
10.98 \\
12.35 \\
12.56 \\
12.03 \\
4.49 \\
-4.12 \\
-1.56 \\
2.27 \\
2.44 \\
-1.79 \\
1.29 \\
2.30\end{array}$ & 5.23 \\
\hline $\begin{array}{l}154.579 \\
154.560 \\
154.542 \\
154.497 \\
154.449 \\
154.410 \\
154.388 \\
154.361 \\
154.267 \\
154.169 \\
154.171 \\
154.007 \\
154.000 \\
153.792 \\
153.574 \\
153.338 \\
152.860 \\
152.842 \\
154.808 \\
155.046 \\
155.143 \\
155.280 \\
155.514 \\
155.737 \\
155.740 \\
155.752 \\
154.221 \\
154.579 \\
154.409\end{array}$ & $\begin{array}{r}12.98 \\
12.51 \\
12.26 \\
11.87 \\
11.57 \\
11.38 \\
11.29 \\
11.18 \\
10.86 \\
10.59 \\
10.49 \\
10.22 \\
10.21 \\
9.83 \\
9.40 \\
9.18 \\
8.66 \\
8.65 \\
15.28 \\
15.29 \\
15.29 \\
15.30 \\
15.30 \\
15.29 \\
15.30 \\
15.30 \\
15.31 \\
14.29 \\
15.90\end{array}$ & $\begin{array}{l}.7700 \\
.2825 \\
.2152 \\
.1524 \\
.1256 \\
.1206 \\
.1058 \\
.1134 \\
.0906 \\
.0687 \\
.0870 \\
.0723 \\
.0707 \\
.0565 \\
.0517 \\
.0476 \\
.0405 \\
.0457 \\
.1295 \\
.1110 \\
.1213 \\
.1029 \\
.0950 \\
.0844 \\
.0978 \\
.0922 \\
.0851 \\
.6580 \\
.1265\end{array}$ & $\begin{array}{l}.4065 \\
.1839 \\
.1432 \\
.1057 \\
.0893 \\
.0813 \\
.0779 \\
.0744 \\
.0659 \\
.0602 \\
.0585 \\
.0541 \\
.0538 \\
.0489 \\
.0452 \\
.0423 \\
.0380 \\
.0379 \\
.0974 \\
.0861 \\
.0832 \\
.0797 \\
.0754 \\
.0724 \\
.0723 \\
.0721 \\
.0676 \\
.3889 \\
.0828\end{array}$ & $\begin{array}{l}.3635 \\
.0986 \\
.0720 \\
.0467 \\
.0363 \\
.0393 \\
.0279 \\
.0390 \\
.0247 \\
.0085 \\
.0285 \\
.0182 \\
.0169 \\
.0076 \\
.0065 \\
.0053 \\
.0025 \\
.0078 \\
.0321 \\
.0249 \\
.0381 \\
.0232 \\
.0196 \\
.0120 \\
.0255 \\
.0201 \\
.0175 \\
.2691 \\
.0437\end{array}$ & $\begin{array}{r}47.21 \\
34.89 \\
33.47 \\
30.61 \\
28.93 \\
32.82 \\
26.41 \\
34.44 \\
27.31 \\
12.40 \\
32.78 \\
25.22 \\
23.83 \\
13.54 \\
12.56 \\
11.21 \\
6.14 \\
17.11 \\
24.76 \\
22.43 \\
31.44 \\
22.53 \\
20.56 \\
14.21 \\
26.07 \\
21.76 \\
20.55 \\
40.89 \\
34.57\end{array}$ & 7.61 \\
\hline
\end{tabular}


from the variables tabulated. If we solve eq (19) for $C_{p}$ and substitute into eq (20) the relation for the Prandtl number becomes

$$
\operatorname{Pr}=\frac{\eta}{\rho \alpha} \text {. }
$$

As an example we use eq (21) to determine $\mathrm{Pr}$ for the saturated liquid at $100 \mathrm{~K}$

$$
\operatorname{Pr}=\frac{1542.3 \times 10^{-6}}{1.0907 \times 0.000723}=1.956
$$

Values for $\eta, \rho$, and $\alpha$ are taken from table 4 . Because the viscosity is tabulated in $\mu \mathrm{g} / \mathrm{cm} \mathrm{s}$ rather than $\mathrm{Pa} \mathrm{s}$, no unit conversions are required, and $\mathrm{Pr}$ is dimensionless as expected.

6. Description of the Data Tables

The following tables of values for the transport properties are presented in this report:

Table 4. Transport properties of oxygen for saturated liquid and vapor, SI units.

Table 5. Transport properties of oxygen, isobars, SI units.

Table 6. Transport properties of oxygen for saturated liquid and vapor, Engr. units.

Table 7. Transport properties of oxygen, isobars, Engr. units.

Table entries of temperature and pressure are chosen to correspond to the values given in NASA RP 1011 [5] so that the present tables can be thought of as an augmentation or extension of the earlier ones. Slight differences in the vapor pressure and density entries may be evident; they occur because the tables of NASA RP 1011 [5] were prepared using a polynomial PVT surface, while the present tables utilize a modified Benedict-Webb-Rubin equation of state [7]. It was not possible to use the polynomial PVT surface because that program specifically excludes a portion of the PVT surface close to the critical point. Conversions to other SI units and to units normally used in applied problems are given in appendix $C$. The tables were calculated using the programs listed in appendices $A$ and $B$. The new programs given in appendix $B$ for the thermal conductivity were written to be compatible with the equation of state package of reference [7]. The primary variables internal to the computer programs of reference [7] are pressure in atmospheres, density in moles/liter, and temperature in 
kelvins. The changes required in the program set of reference [7] to implement the new thermal conductivity calculation are listed in appendix $B$. The changes are minimal, and they are indicated in appendix $B$ by arrows. Branching occurs on the value of the molecular weight. We note that the dilute gas value for thermal conductivity, FUNCTION DILV(T), ENTRY DILT of reference [7] remains unchanged. The number of digits given in tables 4-7 should not be construed to be indicative of the accuracy of a quantity. Most of the properties in the tables range in value over several orders of magnitude, making it necessary to print more digits than is needed at one end of the range. If a given property is varying very slowly it may require digits beyond those necessary for accuracy to show the direction of the change. As an extreme case consider the triple point, the first two lines in table 4. The triple point pressure is not known to be better than about two digits, yet the saturated liquid density differs by five orders of magnitude from that of the vapor.

\section{Error Estimates}

Viscosity: The most recent experimental measurements published for a wide range of the viscosity surface are those by Haynes [4]. These new measurements cover temperatures from 75 to $300 \mathrm{~K}$ with pressures to $34 \mathrm{MPa}$. The correlation [3] represents the 175 experimental points with a RMS deviation of 1 percent ( 1 o) where the maximum and minimum deviations are +3 and -3 percent. Thus the uncertainty in viscosity for the tables presented here is approximately 1 percent for the dilute gas rising to 3 percent at pressures of $34 \mathrm{MPa}$. The uncertainty is expected to increase to no more than 10 percent for pressures up to $100 \mathrm{MPa}$.

Thermal Conductivity: The uncertainty of the dilute gas values is approximately 1 percent at room temperature and 1 atmosphere pressure, rising to 5 percent in going down to the triple point. The accuracy of the tables is expected to be 1.5 percent $(1 \sigma)$ over much of the surface, as established by the fit of the correlating surface [8]. This accuracy degrades to around 6 percent at $77 \mathrm{~K}$ and zero density and to around 6 percent in the region covering the critical enhancement at $159 \mathrm{~K}$. For temperatures closer to critical the calculated values may be in error by as much as 30 percent. Extrapolation of the thermal conductivity surface of [8] for temperatures higher than about $340 \mathrm{~K}$ has not been examined.

Thermal Diffusivity: The uncertainty is estimated to be 5 percent, except for the critical region. At the critical point the thermal diffusivity is 
expected to go to zero, even though both thermal conductivity and specific heat at constant pressure diverge strongly. In the near critical region ( $T_{c} \pm 3$ percent; $\rho_{c} \pm 30$ percent) the uncertainties are as large as 30 percent when compared to experiment.

\section{Summary}

This report presents tables of viscosity, thermal conductivity, and thermal diffusivity of oxygen, as a function of temperature and pressure from the triple point to $320 \mathrm{~K}$ with pressures to $100 \mathrm{MPa}$. Values of the viscosity are calculated from a previous correlation. Values of the thermal conductivity are calculated from a new correlation which combines new experimental measurements of the thermal conductivity and new experimental measurements of the thermal diffusivity in the critical region. 


\section{References}

1. McCarty, R. D.; and Weber, L. A.: Thermophysical Properties of Oxygen from the Freezing Liquid Line to $600 \mathrm{R}$ for Pressures to 5000 psia. (NBS-TN-384, National Bureau of Standards.) NASA CR-121739, Ju1y 1971.

2. Roder, H. M.; and Weber, L. A.: ASRDI Oxygen Technology Survey. Volume I: Thermophysical Properties. NASA SP-3071, 1972.

3. Hanley, H. J. M.; McCarty, R. D.; and Haynes, W. M.: The Viscosity and Thermal Conductivity Coefficients for Dense Gaseous and Liquid Argon, Krypton, Xenon, Nitrogen and Oxygen. J. Phys. Chem. Ref. Data, vol. 3, no. 4, 1974, pp. 979-1017.

4. Haynes, W. M.: Measurements of the Viscosity of Compressed Gaseous and Liquid 0xygen. Physica, vol. 89A, 1977, pp. 569-582.

5. Weber, L. A.: Thermodynamic and Related Properties of Oxygen from the Triple Point to $300 \mathrm{~K}$ at Pressures to 1000 bar. NASA RP-1011, 1977.

6. Weber, L. A.: A Modified Benedict-Webb-Rubin Equation of State for Gaseous and Liquid 0xygen. (NBSIR-78-882, National Bureau of Standards.) NASA CR-157861, April 1978.

7. McCarty, R. D.: Interactive FORTRAN IV Computer Programs for the Thermodynamic and Transport Properties of Selected Cryogens (Fluids Pack). (NBS-TN-1025, National Bureau of Standards.) NASA CR-164016, Oct. 1980.

8. Roder, H. M.: The Thermal Conductivity of Oxygen (Submitted to J. Res. Nat. Bur. Stands.)

9. Weber, L. A.: Thermal Conductivity of Oxygen in the Critical Region. (Submitted to Intern. J. Thermophysics.)

10. Sengers, J. V.; Basu, R. S.; and Levelt Sengers, J. M. H.: Representative Equations for the Thermodynamic and Transport Properties of Fluids Near the Gas-Liquid Critical Point. NASA CR-3424, May 1981. 
Table 4. Transport propertles of oxygen for saturated liquld and Vapor, si UnIts

\begin{tabular}{|c|c|c|c|c|c|c|c|c|c|c|c|}
\hline $\begin{array}{c}\text { Tenp. } \\
k\end{array}$ & $\begin{array}{c}\text { Pressure } \\
\text { MPa }\end{array}$ & $\begin{array}{l}\text { Denslty } \\
\text { g/en**3 }\end{array}$ & $\begin{array}{l}\text { Thermal } \\
\text { Cond. } \\
\text { w/m.k }\end{array}$ & $\begin{array}{c}\text { VIscosity } \\
\text { miero- } \\
\text { g/cn.s }\end{array}$ & $\begin{array}{c}\text { Thermal } \\
\text { Diffusivity } \\
\text { em**2/s }\end{array}$ & $\begin{array}{c}\text { Temp. } \\
k\end{array}$ & $\begin{array}{l}\text { Pressure } \\
\text { No: }\end{array}$ & $\begin{array}{l}\text { Density } \\
0 / \mathrm{cm} * 33\end{array}$ & $\begin{array}{l}\text { Thermal } \\
\text { Cond. } \\
\text { W/m.k }\end{array}$ & $\begin{array}{c}\text { VIscosity } \\
\text { alcro- } \\
\text { g/chis }\end{array}$ & $\begin{array}{c}\text { Thermal } \\
\text { Diffusivity } \\
\text { com* } * 2 / s\end{array}$ \\
\hline $\begin{array}{l}54.359 \\
54.359\end{array}$ & $\begin{array}{l}.00015 \\
.00015\end{array}$ & $\begin{array}{r}1.306190 \\
.000010\end{array}$ & $\begin{array}{l}.20356 \\
.00408\end{array}$ & $\begin{array}{r}4852.3 \\
36.3\end{array}$ & $\begin{array}{r}.000937 \\
4.270533\end{array}$ & $\begin{array}{l}106 . \\
106 .\end{array}$ & $\begin{array}{l}.4081 \\
.4081\end{array}$ & $\begin{array}{r}1.05815 \\
.01627\end{array}$ & $\begin{array}{l}.12814 \\
.00999\end{array}$ & $\begin{array}{r}1344.8 \\
80.4\end{array}$ & $\begin{array}{l}.000685 \\
.005636\end{array}$ \\
\hline $\begin{array}{l}56 . \\
56 .\end{array}$ & $\begin{array}{l}.00024 \\
.00024\end{array}$ & $\begin{array}{r}1.299003 \\
.000017\end{array}$ & $\begin{array}{l}.20050 \\
.00436\end{array}$ & $\begin{array}{r}4677.6 \\
38.0\end{array}$ & $\begin{array}{r}.000927 \\
2.860294\end{array}$ & $\begin{array}{l}108 \\
108\end{array}$ & $\begin{array}{r}.4722 \\
.4722\end{array}$ & $\begin{array}{r}1.04690 \\
.01867\end{array}$ & $\begin{array}{r}.12528 \\
.01025\end{array}$ & $\begin{array}{r}1285.6 \\
82.2\end{array}$ & $\begin{array}{l}.000671 \\
.004945\end{array}$ \\
\hline $\begin{array}{l}58 . \\
58 .\end{array}$ & $\begin{array}{l}.00043 \\
.00043\end{array}$ & $\begin{array}{r}1.290220 \\
.000028\end{array}$ & $\begin{array}{l}.19693 \\
.00467\end{array}$ & $\begin{array}{r}4462.3 \\
39.9\end{array}$ & $\begin{array}{r}.000915 \\
1.797919\end{array}$ & $\begin{array}{l}110 . \\
110 .\end{array}$ & $\begin{array}{l}.5434 \\
.5434\end{array}$ & $\begin{array}{r}1.03543 \\
.02134\end{array}$ & $\begin{array}{l}.12241 \\
.01051\end{array}$ & $\begin{array}{r}1229.4 \\
84.0\end{array}$ & $\begin{array}{l}.000657 \\
.004352\end{array}$ \\
\hline $\begin{array}{l}60 . \\
60 .\end{array}$ & $\begin{array}{l}.00073 \\
.00073\end{array}$ & $\begin{array}{r}1.281405 \\
.000047\end{array}$ & $\begin{array}{l}.19353 \\
.00495\end{array}$ & $\begin{array}{r}4248.2 \\
41.8\end{array}$ & $\begin{array}{r}.000905 \\
1.161530\end{array}$ & $\begin{array}{l}112 . \\
112 .\end{array}$ & $\begin{array}{l}.6222 \\
.6222\end{array}$ & $\begin{array}{r}1.02371 \\
.02428\end{array}$ & $\begin{array}{l}.11951 \\
.01078\end{array}$ & $\begin{array}{r}1175.9 \\
85.8\end{array}$ & $\begin{array}{l}.000642 \\
.003840\end{array}$ \\
\hline $\begin{array}{l}62 . \\
62 .\end{array}$ & $\begin{array}{l}.00119 \\
.00119\end{array}$ & $\begin{array}{r}1.272552 \\
.000074\end{array}$ & $\begin{array}{l}.19026 \\
.00520\end{array}$ & $\begin{array}{r}4038.2 \\
43.6\end{array}$ & $\begin{array}{l}.000896 \\
.770926\end{array}$ & $\begin{array}{l}114 . \\
114 .\end{array}$ & $\begin{array}{l}.7090 \\
.7090\end{array}$ & $\begin{array}{r}1.01173 \\
.02753\end{array}$ & $\begin{array}{l}.11659 \\
.01106\end{array}$ & $\begin{array}{r}1124.8 \\
87.7\end{array}$ & $\begin{array}{l}.000626 \\
.003395\end{array}$ \\
\hline $\begin{array}{l}64 . \\
64 .\end{array}$ & $\begin{array}{l}.00188 \\
.00188\end{array}$ & $\begin{array}{r}1.263634 \\
.000113\end{array}$ & $\begin{array}{r}.18720 \\
.00543\end{array}$ & $\begin{array}{r}3934.4 \\
45.4\end{array}$ & $\begin{array}{l}.000888 \\
.525087\end{array}$ & $\begin{array}{l}116 . \\
116 .\end{array}$ & $\begin{array}{r}.8043 \\
.8043\end{array}$ & $\begin{array}{r}.99946 \\
.03111\end{array}$ & $\begin{array}{r}.11365 \\
.01135\end{array}$ & $\begin{array}{r}1076.0 \\
89.6\end{array}$ & $\begin{array}{l}.000610 \\
.003006\end{array}$ \\
\hline 66. & $\begin{array}{l}.00288 \\
.00288\end{array}$ & $\begin{array}{r}1.254707 \\
.000168\end{array}$ & $\begin{array}{l}.18404 \\
.00565\end{array}$ & $\begin{array}{r}3638.3 \\
47.2\end{array}$ & $\begin{array}{r}.000879 \\
.366477\end{array}$ & $\begin{array}{l}118 . \\
118 .\end{array}$ & $\begin{array}{r}.9085 \\
.9085\end{array}$ & $\begin{array}{l}.98687 \\
.03506\end{array}$ & $\begin{array}{l}.11069 \\
.01165\end{array}$ & $\begin{array}{r}1029.4 \\
91.6\end{array}$ & $\begin{array}{l}.000593 \\
.002665\end{array}$ \\
\hline $\begin{array}{l}68 . \\
68 .\end{array}$ & $\begin{array}{l}.00429 \\
.00429\end{array}$ & $\begin{array}{r}1.245706 \\
.000244\end{array}$ & $\begin{array}{r}.18105 \\
.00586\end{array}$ & $\begin{array}{r}3450.8 \\
49.8\end{array}$ & $\begin{array}{l}.000871 \\
.261671\end{array}$ & $\begin{array}{l}120 . \\
120 .\end{array}$ & $\begin{array}{l}1.0222 \\
1.0222\end{array}$ & $\begin{array}{r}.97392 \\
.03939\end{array}$ & $\begin{array}{r}.10770 \\
.01196\end{array}$ & $\begin{array}{r}984.6 \\
93.7\end{array}$ & $\begin{array}{l}.000575 \\
.002365\end{array}$ \\
\hline $\begin{array}{l}70 . \\
70 .\end{array}$ & $\begin{array}{l}.00625 \\
.00625\end{array}$ & $\begin{array}{r}1.236647 \\
.000345\end{array}$ & $\begin{array}{r}.17813 \\
.00607\end{array}$ & $\begin{array}{r}3272.3 \\
50.5\end{array}$ & $\begin{array}{l}.000863 \\
.190824\end{array}$ & $\begin{array}{l}122 . \\
122 .\end{array}$ & $\begin{array}{l}1.1458 \\
1.1658\end{array}$ & $\begin{array}{l}.96059 \\
.04416\end{array}$ & $\begin{array}{l}.10468 \\
.01230\end{array}$ & $\begin{array}{r}941.6 \\
95.8\end{array}$ & $\begin{array}{l}.000557 \\
.002099\end{array}$ \\
\hline $\begin{array}{l}72 . \\
72\end{array}$ & $\begin{array}{l}.00889 \\
.00889\end{array}$ & $\begin{array}{r}1.227527 \\
.000478\end{array}$ & $\begin{array}{l}.17526 \\
.00627\end{array}$ & $\begin{array}{r}3103.0 \\
52.1\end{array}$ & $\begin{array}{l}.000855 \\
.141898\end{array}$ & $\begin{array}{l}124 . \\
124 .\end{array}$ & $\begin{array}{l}1.2797 \\
1.2797\end{array}$ & $\begin{array}{l}.94683 \\
.04940\end{array}$ & $\begin{array}{l}.10164 \\
.01264\end{array}$ & $\begin{array}{r}900.2 \\
98.0\end{array}$ & $\begin{array}{l}.000538 \\
.001662\end{array}$ \\
\hline $\begin{array}{l}74 . \\
74\end{array}$ & $\begin{array}{r}.01240 \\
.01240\end{array}$ & $\begin{array}{r}1.218340 \\
.000650\end{array}$ & $\begin{array}{r}.17243 \\
.00647\end{array}$ & $\begin{array}{r}2943.0 \\
53.8\end{array}$ & $\begin{array}{l}.000847 \\
.107424\end{array}$ & $\begin{array}{l}126 \\
126\end{array}$ & $\begin{array}{l}1.4245 \\
1.4245\end{array}$ & $\begin{array}{l}.93260 \\
.05515\end{array}$ & $\begin{array}{l}.09857 \\
.01302\end{array}$ & $\begin{array}{l}860 \cdot 3 \\
100 \cdot 3\end{array}$ & $\begin{array}{l}.000518 \\
.001651\end{array}$ \\
\hline $\begin{array}{l}76 . \\
76 .\end{array}$ & $\begin{array}{r}.01695 \\
.01695\end{array}$ & $\begin{array}{r}1.209083 \\
.000866\end{array}$ & $\begin{array}{l}.16964 \\
.00667\end{array}$ & $\begin{array}{r}2791.9 \\
55.4\end{array}$ & $\begin{array}{l}.000839 \\
.082673\end{array}$ & $\begin{array}{l}128 . \\
128 .\end{array}$ & $\begin{array}{l}1.5807 \\
1.5807\end{array}$ & $\begin{array}{l}.91782 \\
.06149\end{array}$ & $\begin{array}{l}.09548 \\
.01342\end{array}$ & $\begin{array}{l}021.6 \\
102.7\end{array}$ & $\begin{array}{l}.000497 \\
.001462\end{array}$ \\
\hline $\begin{array}{l}78 . \\
78 .\end{array}$ & $\begin{array}{l}.02276 \\
.02276\end{array}$ & $\begin{array}{r}1.199753 \\
.001137\end{array}$ & $\begin{array}{l}.16687 \\
.00687\end{array}$ & $\begin{array}{r}2649.5 \\
57.0\end{array}$ & $\begin{array}{l}.000831 \\
.064591\end{array}$ & $\begin{array}{l}130 . \\
130 .\end{array}$ & $\begin{array}{l}1.7488 \\
1.7488\end{array}$ & $\begin{array}{l}.90245 \\
.06847\end{array}$ & $\begin{array}{l}.09235 \\
.01385\end{array}$ & $\begin{array}{l}784.1 \\
105.2\end{array}$ & $\begin{array}{l}.000475 \\
.001292\end{array}$ \\
\hline $\begin{array}{l}80 . \\
80 .\end{array}$ & $\begin{array}{l}.03009 \\
.03009\end{array}$ & $\begin{array}{r}1.190345 \\
.0014 n 9\end{array}$ & $\begin{array}{l}.16411 \\
.00708\end{array}$ & $\begin{array}{r}2515.5 \\
58.7\end{array}$ & $\begin{array}{l}.000823 \\
.051164\end{array}$ & $\begin{array}{l}132 . \\
132 .\end{array}$ & $\begin{array}{l}1.9294 \\
1.9294\end{array}$ & $\begin{array}{l}.88640 \\
.07618\end{array}$ & $\begin{array}{l}.08920 \\
.01433\end{array}$ & $\begin{array}{l}747.6 \\
107.9\end{array}$ & $\begin{array}{l}.000453 \\
.001138\end{array}$ \\
\hline $\begin{array}{l}82, \\
82 \text {. }\end{array}$ & $\begin{array}{r}.03919 \\
.03919\end{array}$ & $\begin{array}{r}1.180854 \\
.001872\end{array}$ & $\begin{array}{l}.16137 \\
.00728\end{array}$ & $\begin{array}{r}2389.5 \\
60.3\end{array}$ & $\begin{array}{l}.000814 \\
.041042\end{array}$ & $\begin{array}{l}134 . \\
134 .\end{array}$ & $\begin{array}{l}2.1229 \\
2.1229\end{array}$ & $\begin{array}{r}.86957 \\
.08472\end{array}$ & $\begin{array}{l}.08602 \\
.01487\end{array}$ & $\begin{array}{l}712.0 \\
110.8\end{array}$ & .000429 \\
\hline $\begin{array}{l}84 . \\
84 .\end{array}$ & $\begin{array}{l}.05035 \\
.05035\end{array}$ & $\begin{array}{r}1.171275 \\
.002356\end{array}$ & $\begin{array}{l}.15864 \\
.00749\end{array}$ & $\begin{array}{r}2271.0 \\
61.9\end{array}$ & $\begin{array}{l}.000806 \\
.033303\end{array}$ & $\begin{array}{l}136 . \\
136 .\end{array}$ & $\begin{array}{l}2.3301 \\
2.3301\end{array}$ & $\begin{array}{l}.85185 \\
.09421\end{array}$ & $\begin{array}{r}.08282 \\
.01549\end{array}$ & $\begin{array}{l}677.2 \\
113.9\end{array}$ & .000404 \\
\hline $\begin{array}{l}86 . \\
86 .\end{array}$ & $\begin{array}{l}.06387 \\
.06387\end{array}$ & $\begin{array}{r}1.161604 \\
.007931\end{array}$ & $\begin{array}{l}.15590 \\
.00770\end{array}$ & $\begin{array}{r}2159.5 \\
63.5\end{array}$ & $\begin{array}{l}.000797 \\
.027309\end{array}$ & $\begin{array}{l}138 . \\
138 .\end{array}$ & $\begin{array}{l}2.5514 \\
2.5514\end{array}$ & $\begin{array}{r}.83309 \\
.10481\end{array}$ & $\begin{array}{l}.07960 \\
.01621\end{array}$ & $\begin{array}{l}642.9 \\
117.3\end{array}$ & $\begin{array}{l}.000378 \\
.000759\end{array}$ \\
\hline $\begin{array}{l}88 . \\
88 .\end{array}$ & $\begin{array}{l}.08007 \\
.08007\end{array}$ & $\begin{array}{r}1.151835 \\
.003607\end{array}$ & $\begin{array}{l}.15317 \\
.00791\end{array}$ & $\begin{array}{r}2054.7 \\
65.2\end{array}$ & $\begin{array}{l}.000787 \\
.022609\end{array}$ & $\begin{array}{l}240 . \\
140 .\end{array}$ & $\begin{array}{l}2.7875 \\
2.7875\end{array}$ & $\begin{array}{r}.81308 \\
.11674\end{array}$ & $\begin{array}{l}.07638 \\
.01706\end{array}$ & $\begin{array}{l}608.9 \\
121.1\end{array}$ & $\begin{array}{l}.000350 \\
.000654\end{array}$ \\
\hline $\begin{array}{l}90 . \\
90 .\end{array}$ & $\begin{array}{r}.09931 \\
.09931\end{array}$ & $\begin{array}{r}1.141960 \\
.004396\end{array}$ & $\begin{array}{l}.15043 \\
.00812\end{array}$ & $\begin{array}{r}1956.1 \\
66.8\end{array}$ & $\begin{array}{l}.000778 \\
.018882\end{array}$ & $\begin{array}{l}142 . \\
142 .\end{array}$ & $\begin{array}{l}3.0392 \\
3.0392\end{array}$ & $\begin{array}{r}.79155 \\
.13028\end{array}$ & $\begin{array}{l}.07317 \\
.01811\end{array}$ & $\begin{array}{l}575.1 \\
125.3\end{array}$ & $\begin{array}{l}.000320 \\
.000557\end{array}$ \\
\hline 92. & $\begin{array}{r}.12194 \\
.12194\end{array}$ & $\begin{array}{r}1.131973 \\
.005308\end{array}$ & $\begin{array}{l}.14769 \\
.00834\end{array}$ & $\begin{array}{r}1863.3 \\
68.5\end{array}$ & $\begin{array}{l}.000768 \\
.015896\end{array}$ & $\begin{array}{l}144 . \\
144 .\end{array}$ & $\begin{array}{l}3.3072 \\
3.3072\end{array}$ & $\begin{array}{r}.76811 \\
.14584\end{array}$ & $\begin{array}{l}.07002 \\
.01945\end{array}$ & $\begin{array}{l}541.1 \\
130.1\end{array}$ & $\begin{array}{l}.000289 \\
.000467\end{array}$ \\
\hline 94. & $\begin{array}{r}.14833 \\
.14833\end{array}$ & $\begin{array}{r}1.121867 \\
.005357\end{array}$ & $\begin{array}{l}.14494 \\
.00856\end{array}$ & $\begin{array}{r}1775.9 \\
70.1\end{array}$ & $\begin{array}{r}.000757 \\
.013479\end{array}$ & $\begin{array}{l}146 . \\
146 .\end{array}$ & $\begin{array}{l}3.5924 \\
3.5924\end{array}$ & $\begin{array}{l}.74215 \\
.16405\end{array}$ & $\begin{array}{l}.06700 \\
.02121\end{array}$ & $\begin{array}{l}506.4 \\
135.7\end{array}$ & $\begin{array}{l}.000254 \\
.000383\end{array}$ \\
\hline $\begin{array}{l}96 . \\
96 .\end{array}$ & $\begin{array}{r}.17887 \\
.17887\end{array}$ & $\begin{array}{r}1.111632 \\
.007555\end{array}$ & $\begin{array}{l}.14217 \\
.00879\end{array}$ & $\begin{array}{r}1693.5 \\
71.8\end{array}$ & $\begin{array}{l}.000746 \\
.011504\end{array}$ & $\begin{array}{l}148 . \\
148 .\end{array}$ & $\begin{array}{l}3.8958 \\
3.8958\end{array}$ & $\begin{array}{r}.71262 \\
.18598\end{array}$ & $\begin{array}{l}.06423 \\
.02365\end{array}$ & $\begin{array}{l}470.4 \\
142.6\end{array}$ & $\begin{array}{l}.000216 \\
.000302\end{array}$ \\
\hline $\begin{array}{l}98 . \\
98 .\end{array}$ & $\begin{array}{r}.21395 \\
.21395\end{array}$ & $\begin{array}{r}1.101259 \\
.008916\end{array}$ & $\begin{array}{l}.13940 \\
.00902\end{array}$ & $\begin{array}{r}1315.7 \\
73.5\end{array}$ & $\begin{array}{l}.000735 \\
.009878\end{array}$ & $\begin{array}{l}150 . \\
150 .\end{array}$ & $\begin{array}{l}4.2186 \\
4.2186\end{array}$ & $\begin{array}{r}.67747 \\
.21370\end{array}$ & $\begin{array}{l}.06197 \\
.02726\end{array}$ & $\begin{array}{l}431.5 \\
151.5\end{array}$ & $\begin{array}{l}.000171 \\
.000221\end{array}$ \\
\hline $\begin{array}{l}100 . \\
100 .\end{array}$ & $\begin{array}{l}.25399 \\
.25399\end{array}$ & $\begin{array}{r}1.090736 \\
.010452\end{array}$ & $\begin{array}{l}.13661 \\
.00926\end{array}$ & $\begin{array}{r}1542.3 \\
75.2\end{array}$ & $\begin{array}{l}.000723 \\
.008527\end{array}$ & $\begin{array}{l}152 . \\
152 .\end{array}$ & $\begin{array}{l}4.5625 \\
4.5625\end{array}$ & $\begin{array}{r}.63157 \\
.25242\end{array}$ & $\begin{array}{l}.06075 \\
.03324\end{array}$ & $\begin{array}{l}386.2 \\
164.7\end{array}$ & $\begin{array}{l}.000115 \\
.000133\end{array}$ \\
\hline $\begin{array}{l}102 . \\
102 .\end{array}$ & $\begin{array}{l}.29940 \\
.29940\end{array}$ & $\begin{array}{r}1.080053 \\
.012180\end{array}$ & $\begin{array}{l}.13380 \\
.00949\end{array}$ & $\begin{array}{r}1472.8 \\
76.9\end{array}$ & $\begin{array}{l}.000711 \\
.007397\end{array}$ & $\begin{array}{l}154 . \\
154 .\end{array}$ & $\begin{array}{l}4.9305 \\
4.9305\end{array}$ & $\begin{array}{r}.54944 \\
.32914\end{array}$ & $\begin{array}{l}.06376 \\
.05480\end{array}$ & $\begin{array}{l}317.9 \\
193.9\end{array}$ & $\begin{array}{l}.000034 \\
.000035\end{array}$ \\
\hline $\begin{array}{l}104 . \\
104 .\end{array}$ & $\begin{array}{r}.35061 \\
.35061\end{array}$ & $\begin{array}{r}1.069196 \\
.014115\end{array}$ & $\begin{array}{l}.13098 \\
.00974\end{array}$ & $\begin{array}{r}1407.1 \\
78.6\end{array}$ & $\begin{array}{l}.000698 \\
.004444\end{array}$ & $\begin{array}{l}154.581 \\
154.581\end{array}$ & $\begin{array}{l}5.0430 \\
5.0430\end{array}$ & $\begin{array}{r}.54944 \\
.32914\end{array}$ & & & \\
\hline
\end{tabular}


Table 5. Transport Propertles of Oxygen, Isobars, SI Units.

- 1 MP. Isobar

$\begin{array}{cc}\text { Temp. } & \text { Density } \\ k & g / e n * 3\end{array}$

Thermal vise
Cond.
.20358

.20054

.19698

.19031

.18715

.18409

.18110

.17818

.17531

.17248

.16969

.16691
.16416

-16141

.15867

.15593

.15318

.15043

.15034

.00813

.00831

.00851
.00870

.00870

.00889
.00909

.00929

.00948

.00908

.00988

.01008

.01048

.01068

.01088

.01108

.01128

.01147
.01167

.01167
.01187

.01187

.01207

- 01246

.01266

.01285
.01305

.01324

.01343

.01363

.01382

.01401

.01421

.01440

.01439

.01476
.01494

.01539

.01584

.01628

.01672

.01716

.01802

.01802

.01927

.02090

.02090
.02170

.02248

.02248

.02326
.02404

.02404
.02490

.02480

.02556

.02631
.02706
.02782 $\begin{array}{ll}4855.4 & .000937 \\ 4681.7 & .000927\end{array}$

$4466.2 \quad .000916$

$4251.8 \quad .000906$

$4041.6 \quad .000896$

$3837.6 \quad .000888$

$3641.3 \quad .000879$

$\begin{array}{ll}3453.6 & .000871 \\ 3275.0 & .000863\end{array}$

$\begin{array}{ll}3275.0 & .000863 \\ 3105.5 & .000855\end{array}$

$2945.2 \quad .000847$

$2793.9 \quad .000840$

$\begin{array}{ll}2651.4 & .000831 \\ 2517.1 & .000823\end{array}$

$2390.8 \quad .000815$

$\begin{array}{ll}2272.0 & .000806 \\ 2160.3 & .000797\end{array}$

$2055.1 \quad .000787$

$1956.1 \quad .000778$

$1953.0 \quad .000777$

$66.9 \quad .018773$

$60.9 \quad 010778$

$71.5 \quad .02037$

$\begin{array}{ll}71.5 & .021917 \\ 73.0 & .023018\end{array}$

$74.6 \quad .024141$

$76.1 \quad .025286$

$\begin{array}{ll}77.7 & .026452 \\ 79.2 & .027640\end{array}$

$\begin{array}{ll}79.2 & .027640 \\ 80.8 & .028849\end{array}$

$82.3 \quad .030079$

$83.8 \quad .031331$

$86.9 \quad .032604$

$88.4 \quad .035212$

$92.4 \quad .037902$

$92.9 \quad .039278$

$94.4 \quad .040673$

$95.9 \quad .042089$

$97.4 \quad .043523$

$100.4 \quad .046451$

$101.8 \quad .047944$

$\begin{array}{ll}103.3 & .049457 \\ 104.8 & .050988\end{array}$

$106.2 \quad .052539$

107.7

109.2

110.6

112.1

213.5

114.9

116.4

119.2

.054110

.055700

.058946

000604

.060604

.062292

.063940
.065589

.067264

122.7
126.2

126.2
129.7

133.1

136.5

139.8

146.4

156.4

.071543

.075939

.080442

.085048

.089753

.099455

.104449

.104479

165.5

171.6

183.6

189.4

195.1
200.7

206.3

212.7

.136331

.167703

.179402

.183814

.196511

.222843

.236472
.250436
Temp. Density
$k \quad g / C m * * 3$

$+\quad 54.382$

$\begin{array}{ll}54.382 & 1.3063 \\ 58 . & 1.2992\end{array}$

$\begin{array}{ll}58 . & 1.2905 \\ 60 . & 1.2817\end{array}$

62. $\quad 1.2728$

64. $\quad 1.2639$

66. 1.2550

68.1 .2460

72. 1.2369

74. 1.2186

$76 . \quad 1.2094$

$\begin{array}{ll}78 . & 1.2001 \\ 80 . & 1.1906\end{array}$

82. $\quad 1.1812$

83. 1.161

B8. 1.1521

90. $\quad 1.1422$

94.1 .132

95. $\quad 1.1117$

+ $97.237 \quad 1.1052$

$\begin{array}{ll}97.237 & .0084 \\ 98 . & .0083\end{array}$

$\begin{array}{ll}98 . & .0083 \\ 100 . & .0081\end{array}$

102.

104

108

10.

112.

114.

116.

120.

.0079

.0077

.0076

.007

.0071

.0070

.0069

.0066

122. .0065

$\begin{array}{ll}124 . & .0064 \\ 126 . & .0063\end{array}$

126. $\quad .0063$

$130^{\circ}$

132.
134.
136.

138

140.

.0061

.0060

.0059
.0058

.0057

.005

$142 . \quad .0055$

$146 . \quad .0054$

148. .0053

$150 . \quad .0052$

152. .0051

154. .0031

158. $\quad .0050$

$\begin{array}{ll}158 . & .0049 \\ 260 . & .0049\end{array}$

$165 . \quad .0047$

170.

175.

180.
185.
190.

195.

210.

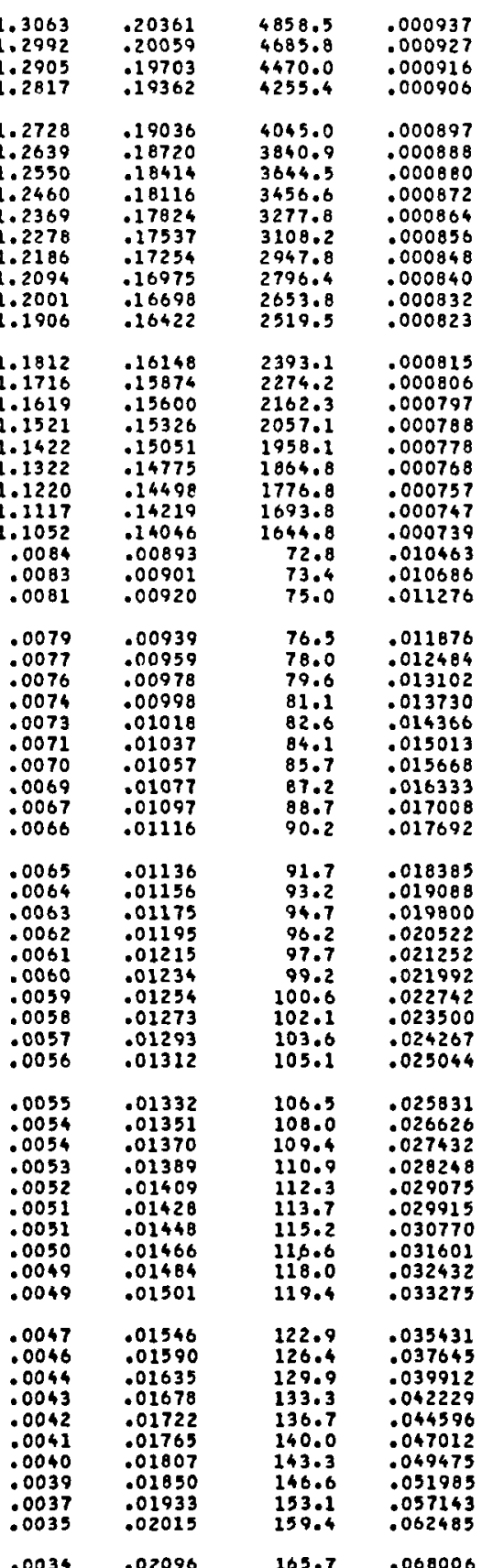

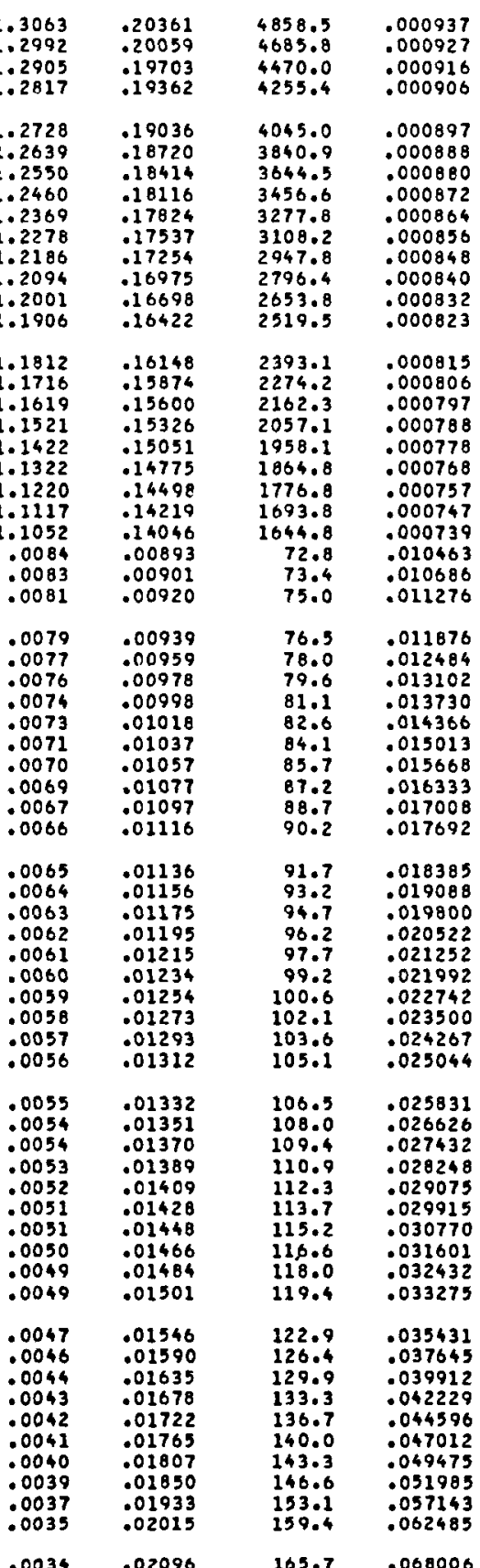

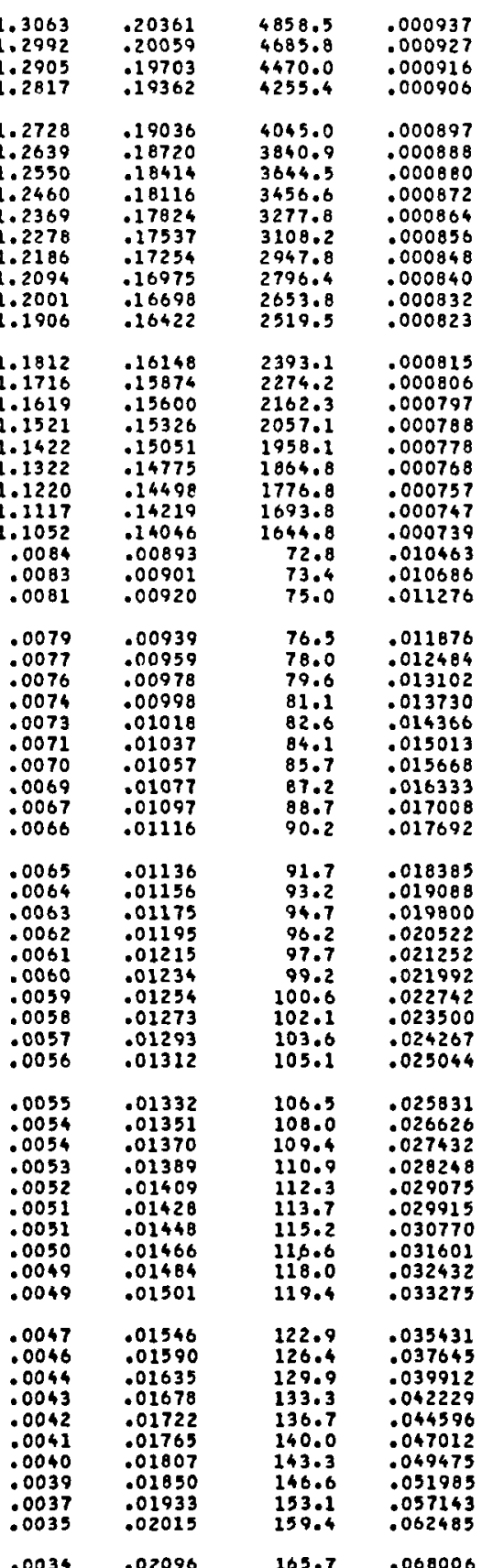

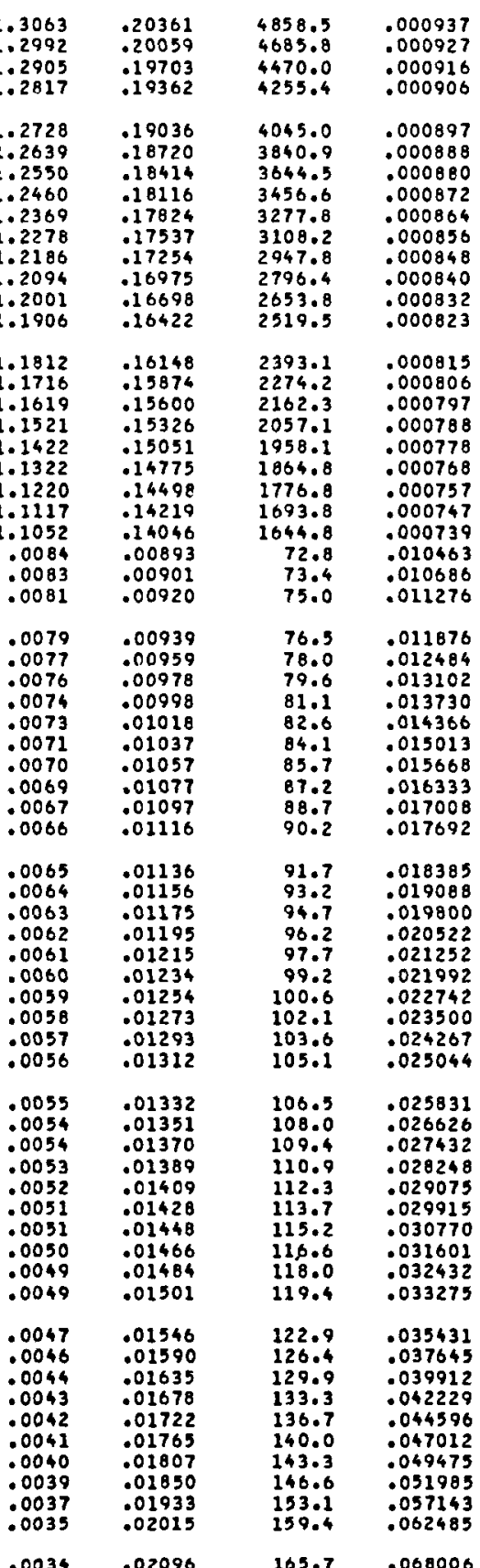

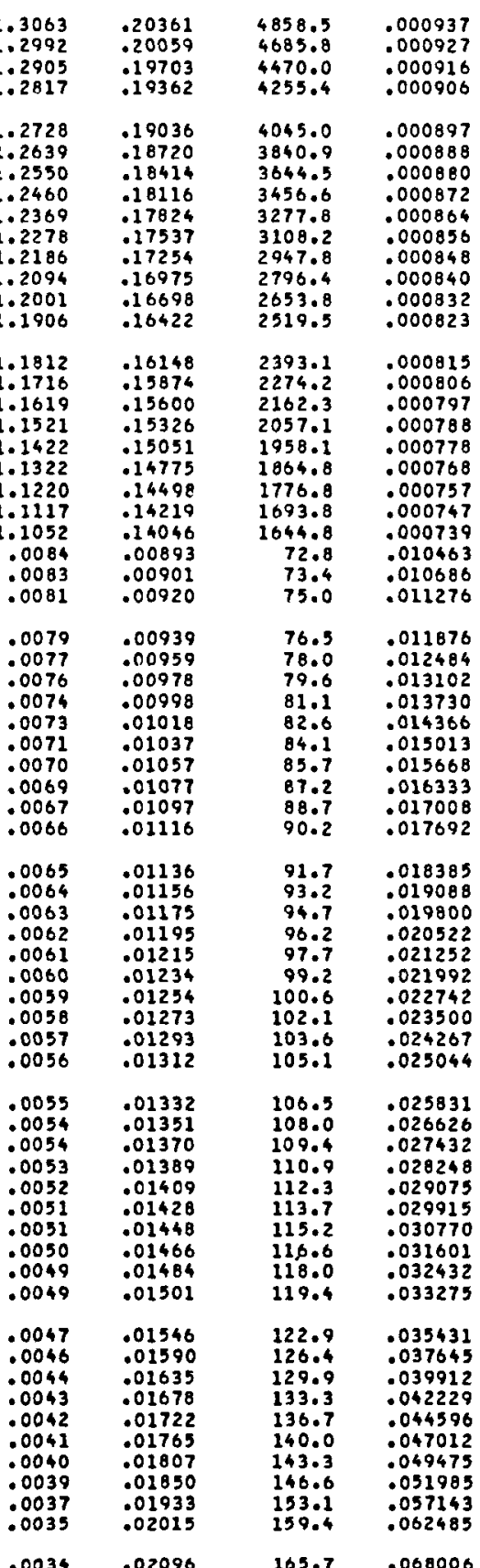

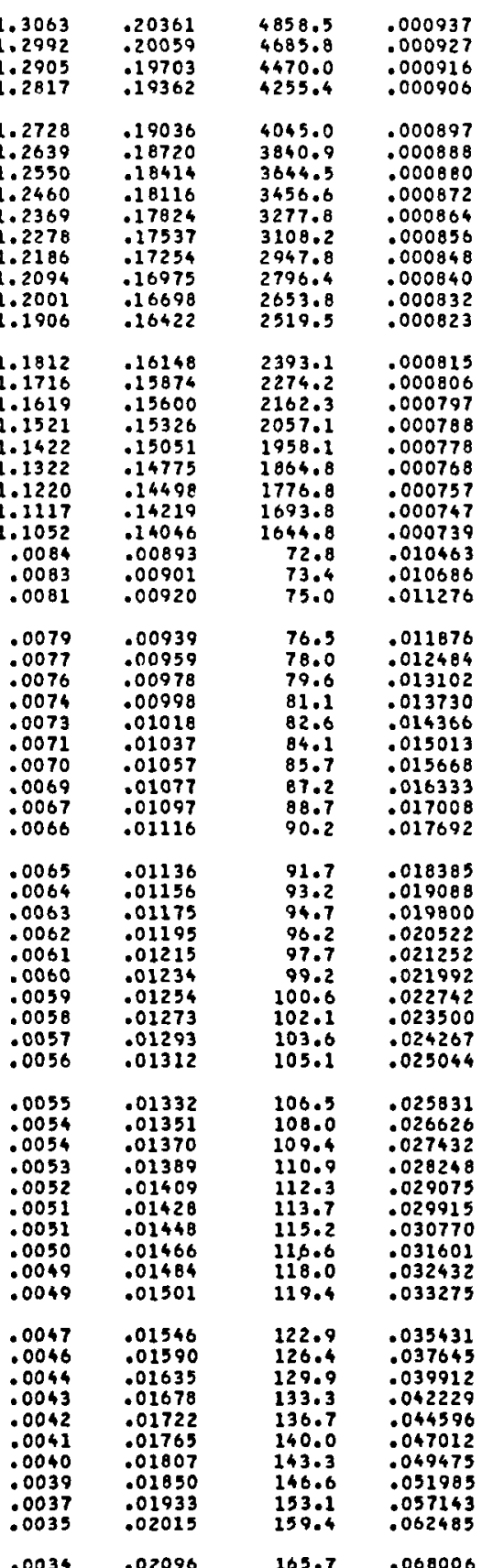

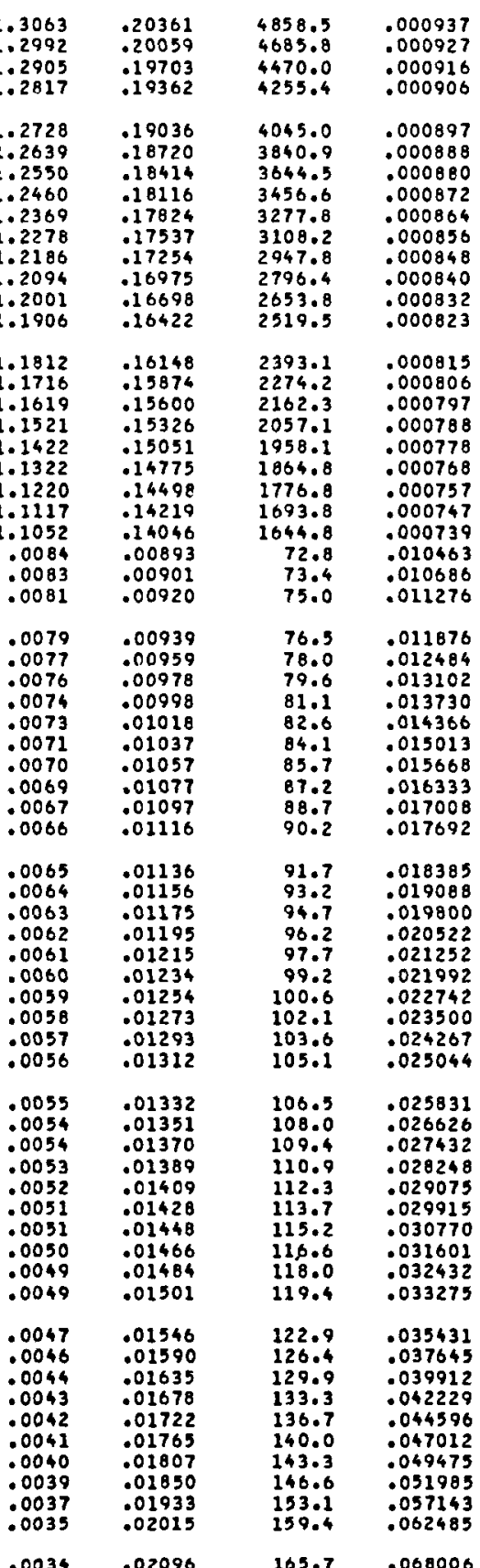

3

cand. mlero- Diffusivity

whok

.19362

1642

.16148

15600

.00920

.00939

00078

.00998

01116

.0127

.01312

.0133

01501

.0034

.0034

.0031

$260 . \quad .0030$

270.

290.

300.

.0028
.0027

.002

.0025

.02096

.02254

.02254

.02332

.02409

.02485 


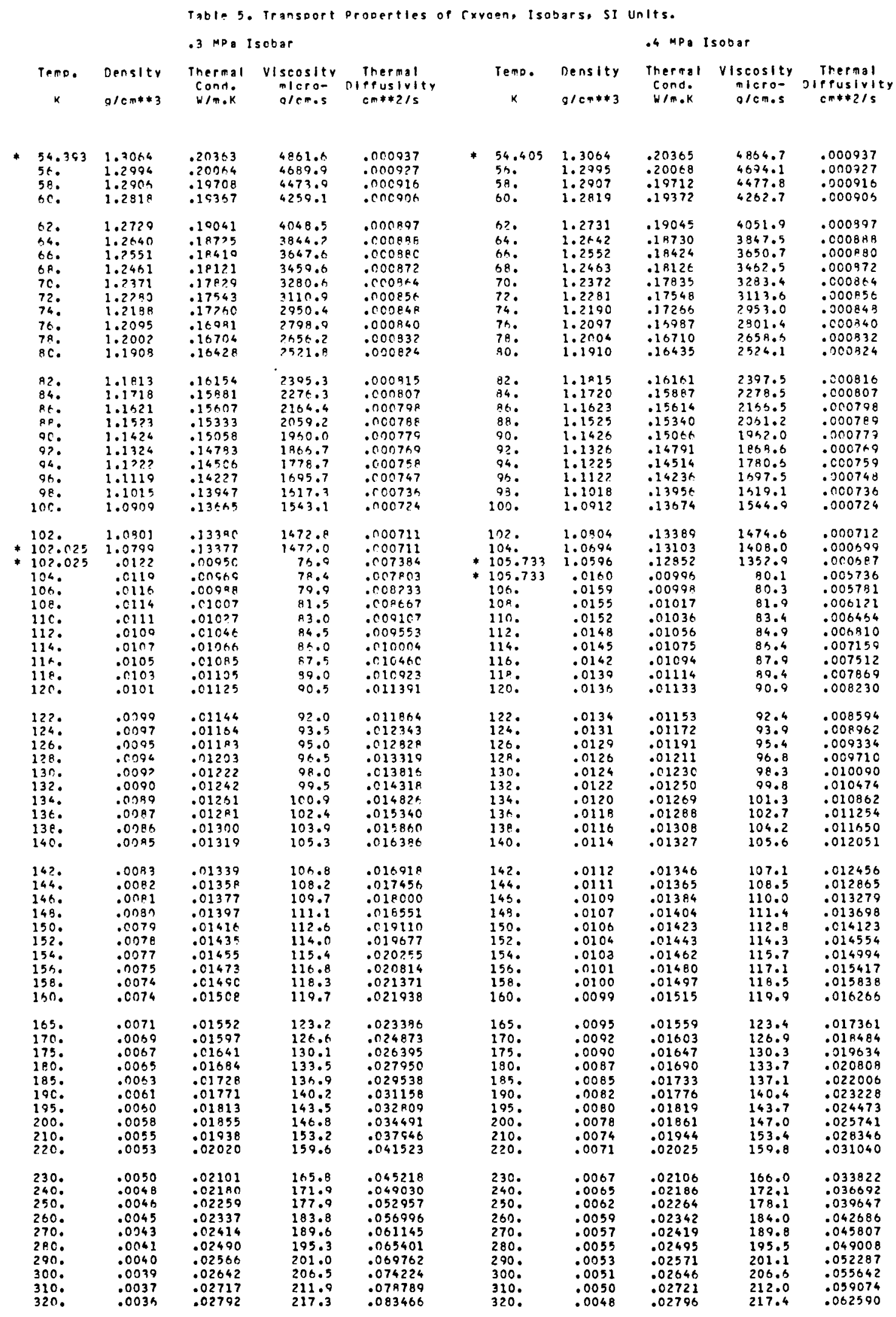

* Two Phase Boundary 
Table 5. Transoort prodeptles of rxygen, Isobars, SI Units.

.5 MP Isntar

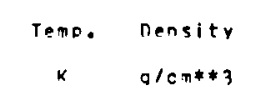

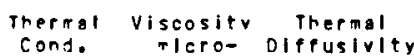

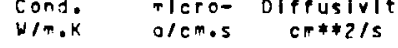

*

$\begin{array}{lll}54.413 & 1.3065 & .20348 \\ 54.0 & 1.2005 & .20073\end{array}$

$\begin{array}{ll}5 A . & 1.200 B \\ A C . & 3.2800\end{array}$

67. 1.2732

t.: 1.2643

$\begin{array}{ll}\text { te. } & 1.2554 \\ \text { hE. } & 1.2444\end{array}$

70. 1.2374

$72 . \quad 2.2 ? 83$

78. $\quad 1.2099$

$8 \mathrm{C.} \quad 1.1017$

82. $\quad 1.1817$

B4. 1.1771

86. 1.142

90.114290

$92 . \quad 1.13 ? 9$

Q4. 1.1125

$\begin{array}{rr}99 . & 1.1020 \\ 100 . & 1.0914\end{array}$

102. 1.0907

$\begin{array}{ll}104 . & 1.0697 \\ 104 . & 1.0585 \\ 10 \% & 1.0470\end{array}$

$108.806 \quad 1.0423$

* 108.806 .0197

11C. .0194

$112 . \quad 0190$

114.

$\begin{array}{ll}114 . & .0181 \\ 11 \% & .0177 \\ 120 . & .0173\end{array}$

127. .0170

124.0 .0160

$179 . \quad 0160$

$130 . \quad .0157$

134. 015 ?

136.0149

$138 . \quad .0146$

$140.016 ?$

14?. $.014 ?$

$\begin{array}{ll}144 . & .0140 \\ 146 . & .0137\end{array}$

148. $\quad .0135$

$150 . \quad .0133$

154.0 .0120

$156 . \quad .0128$

$16 n: \quad .017$

165. .0120

$170 . \quad 0116$

175. $\quad .0113$

180.

185.0 .0106

190. $\quad .0173$

$\begin{array}{ll}195 . & .0100 \\ 200 . & .0078\end{array}$

$\begin{array}{ll}210 . & .0093 \\ 220 . & .0088\end{array}$

$230 . \quad .0084$

$\begin{array}{ll}230 . & .0084 \\ 240 . & .0081 \\ 2500^{\circ} & .0078\end{array}$

$260 . \quad .0074$

$270 . \quad .0072$

290. $\quad .0065$

$300 . \quad .0044$

$310 . \quad .0062$

.0060
.19717
.19377

.10050

.18735

18420
.18132

$.1704 \mathrm{C}$

.17554
.17272

.15093

.16716

.16167

.15894

.15348

15073

14798
$.1457 ?$

$.145 ? 2$

.14244

.13603

.13112

.12531

.12413

. 01035

-c1055

.01084

- 011na

.01173

- c1181

.011200

.01219

.01238

.01257

.01296

- 1315

. 01353

.01373

.01302

.01411

.01450

.01470

.01487

.01504

.01565

.01609

. 1653

.01696

.01739

.01824

.01865

.01949
.02031

.02111

.02191

.02347

.02424

.02500

.02576

.02651

.02726
.02801
.13390
$4631.7 \quad 000017$

$4055.4 \quad 000097$

$2950.7 \quad .000989$

$96 \leq 3.8 \quad$. 000890

$34 \mathrm{h5.5} \quad 0000872$

$3120.7 \quad .000857$

2055.6 . 000940

7803.9 .000941

$\begin{array}{ll}2640.9 & .0 C O B 33 \\ 2520.4 & \text {.nCOB?5 }\end{array}$

$2309.7 \quad .000916$

$2280 . \mathrm{h} .000907$

$2168.6 \quad 000708$

$1944.0 \quad 000780$

$3970.6 \quad 000770$

$\begin{array}{ll}1790.5 & .000775 \\ 1792.5 & .000750\end{array}$

$1609.4 \quad 0000748$

$1520.0 \quad .000737$

$1546.7 \quad .000725$

$\begin{array}{ll}1475.6 & .000713 \\ 1409.7 & .000699\end{array}$

$1346.4 \quad .000686$

$1286.1 \quad .000672$

$1262.5 \quad .000+66$

37.9 .004695

85.3005

0.00543

$08.3 \quad .005735$

$\begin{array}{ll}88.3 & .005735 \\ 80.8 & .00 A 029\end{array}$

$01.3 \quad .003325$

$92.7 \quad .000625$

$94.2 \quad .006926$

$77.2 \quad .007539$

$98.7 \quad .007840$

$100.1 \quad .008162$

$103.0 \quad .008708$

$104.5 \quad .009120$

$105.9 \quad .000446$

$107.4 \quad .000775$

$\begin{array}{ll}108.8 & .010107 \\ 110.3 & .010444\end{array}$

$\begin{array}{ll}110.3 & .010444 \\ 111.7 & .010784\end{array}$

$114.5 \quad .011478$

$116.0 \quad .011835$

$117.4 \quad .012176$

$120.2 \quad .012861$

$123.7 \quad .013743$

$127.1 \quad .014649$

$130.5 \quad .015575$

.016521

$140.6 \quad .018469$

$43.9 \quad .019471$

$147.2 \quad .020490$

$\begin{array}{ll}153.6 & .022584 \\ 159.9 & .024749\end{array}$

$166.1 \quad .026984$

$172.2 \quad .029288$

$184.1 \quad .034090$

$189.9 \quad .036603$

$195.6 \quad .039171$

$201.2 \quad .041801$

$\begin{array}{ll}212.1 & .047243 \\ 217.5 & .050062\end{array}$
$113.1 \quad .011128$

Tend. Density
$k \quad g / 0 m * * 3$

$.6 \mathrm{MPa} I s o b a r$

Thermol viscosity Thermal

$\begin{array}{ll}\text { Cond. } & \text { mlero- Diffusivity } \\ \text { wim.k } & \text { olem.s }\end{array}$

- $5+.428 \quad 1.3066$

56. $\quad 1.2997 \quad .20370$

$58 . \quad 1.2909$

.19722

32. 1.2733

64. 1.2644

66. 1.2555

38. 1.2465

$72 . \quad 1.2284$

74.1 .2193

$\begin{array}{ll}76 . & 1.2100 \\ 78 . & 1.2007\end{array}$

80. $\quad 1.1914$

R2. 1.1819

84. 1.1723

ค9. 1.1529

90. 1.1431

9?. 1.1331

74. $1.123 \mathrm{C}$

9. 99.1127

$\begin{array}{rr}99 . & 1.1023 \\ 100 . & 1.0917\end{array}$

102. $\quad 1.0810$

$\begin{array}{ll}104 . & 1.0700 \\ 106 . & 1.0588 \\ 109 . & 1.0474\end{array}$

$110 . \quad 1.0356$

$+111.457 \quad 1.0269$

112.0233

$\begin{array}{ll}112 . & .0233 \\ 114 . & .0227\end{array}$

116.0 .022

$\begin{array}{ll}118 . & .0216 \\ 120 . & .0212\end{array}$

.19055

.18740

.18435

.18137

.17846

.17277

.16999

.16722
.16448

.16174

.15901

.15355

.15081

.14806

.14252

.13973
.13692

.13408

.13122

.12542

.12542

.12030

.01071

.01076

.01094

.01113

.01132

.01170

122. .0207

$124 . \quad .0203$

.01189

.01208

.01227

.01246

$128 . \quad .0195$

130.0 .0191

134. .0184

$336 . \quad .0181$

138. .0178

.01266

.01304

.01304

.01323

.01361

142. .0172

$144 . \quad .0160$

.01380

.01390

$148 . \quad 0164 \quad .01419$

$\begin{array}{lll}150 . & .0161 & .01438 \\ 152 & .0159 & .01457\end{array}$

$\begin{array}{lll}152 . & .0159 & .01457 \\ 154 . & .0156 & .01478\end{array}$

156. .0154

$158 . \quad .0152$

.01495

.01529

.01572

165. .0145

170 .

175 .

.01615

.01659

.01659

.01702

.01745

.01788

.01872

.01954

.02036

and

$\begin{array}{ll}4870.9 & .000937 \\ 4702.3 & .000928 \\ 4485.5 & .000917 \\ 4270.1 & .000907 \\ 4058.9 & .000898 \\ 3854.0 & .000889 \\ 3656.9 & .000881 \\ 3468.5 & .000873 \\ 3289.1 & .000865 \\ 3119.0 & .000957 \\ 2958.2 & .000849 \\ 2806.4 & .000841 \\ 2663.3 & .000833 \\ 2528.7 & .000825 \\ & \\ 2402.0 & .000816 \\ 2282.8 & .000808 \\ 2170.7 & .000799 \\ 2055.2 & .000790 \\ 1764.0 & .000780 \\ 1872.5 & .000770 \\ 1794.4 & .000760 \\ 1701.2 & .000749 \\ 1522.7 & .000737 \\ 1548.4 & .000726 \\ & \\ 1478.1 & .000713 \\ 1411.5 & .000700 \\ 1348.1 & .000687 \\ 1287.8 & .000572 \\ 1230.4 & .000657 \\ 1190.2 & .000646 \\ 85.3 & .003972 \\ 85.7 & .004040 \\ 87.2 & .004290 \\ 89.7 & .004542 \\ 90.2 & .006795 \\ 91.7 & .005049 \\ & \\ & \\ & \\ & \\ & \end{array}$

$93.1 \quad .005305$

$\begin{array}{ll}74.6 & .005564 \\ 96.1 & .005824\end{array}$

$97.5 \quad .000086$

$99.0 \quad .006350$

$100.5 \quad .006617$

$101.9 \quad .006886$

$103.4 \quad .007157$

$104.8 \quad .007430$

$107.7 \quad .007985$ 
Tarle 5. Transoopt Propertles of Oxygen, Isobars, SI Units.

.7 MP. IsODar

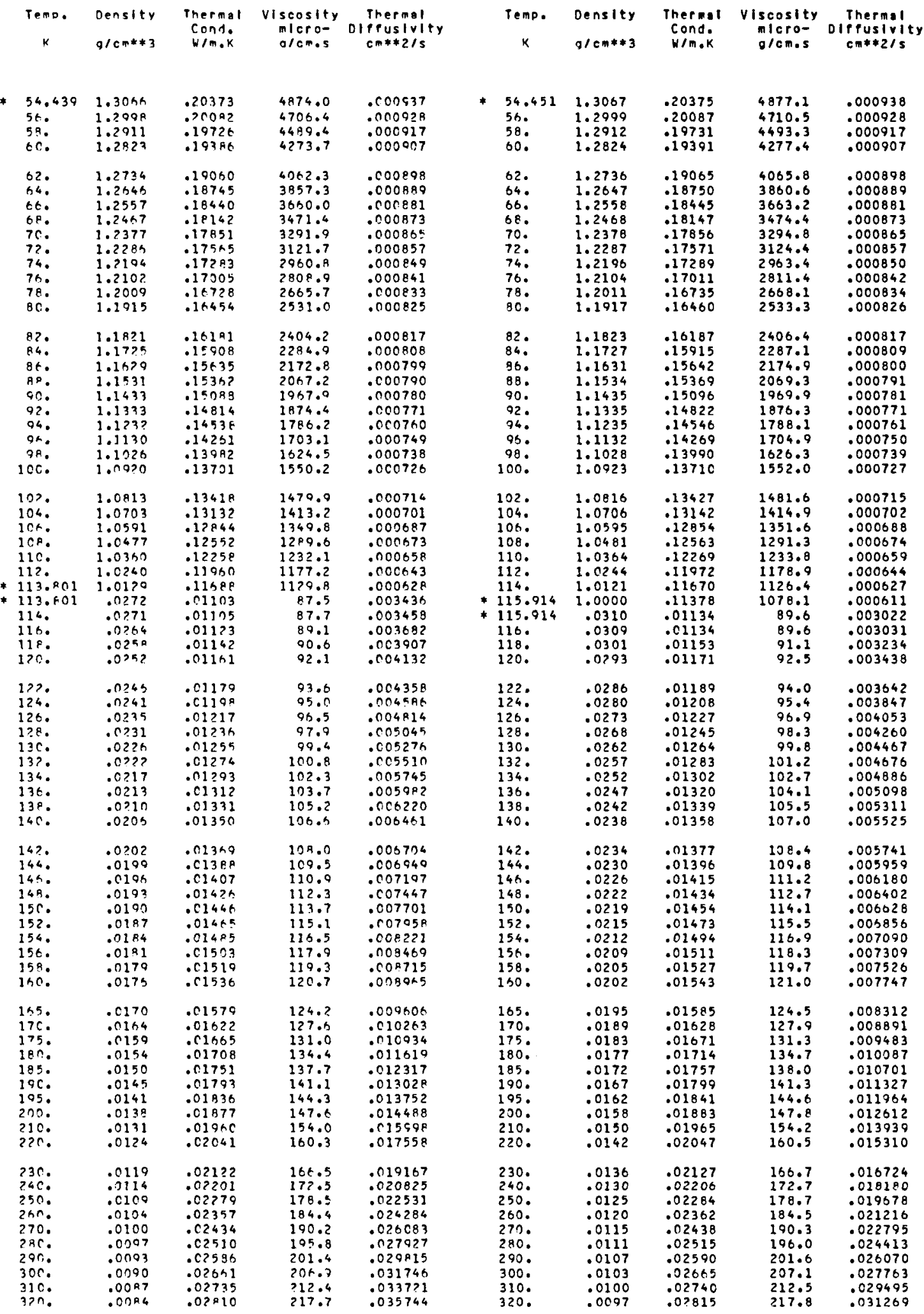


Table 3. Transport Properties of Cxyoen. Isobars, SI UnIts.

- 9 MPa Isohor

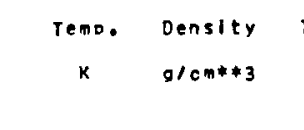

$+$

\begin{tabular}{|c|c|c|c|c|c|c|c|c|c|}
\hline $\begin{array}{l}54.462 \\
56 . \\
58 . \\
60 .\end{array}$ & $\begin{array}{l}1.3068 \\
1.3001 \\
1.2913 \\
1.2825\end{array}$ & $\begin{array}{l}.20377 \\
.20091 \\
.19736 \\
.19396\end{array}$ & $\begin{array}{l}4890.2 \\
4714.6 \\
4497.2 \\
4291.0\end{array}$ & $\begin{array}{l}.000938 \\
.000928 \\
.000917 \\
.000907\end{array}$ & $\begin{array}{l}\quad 54.474 \\
56 . \\
58 . \\
60 .\end{array}$ & $\begin{array}{l}1.3068 \\
1.3002 \\
1.2914 \\
1.2826\end{array}$ & $\begin{array}{l}.20380 \\
.20096 \\
.19740 \\
.19401\end{array}$ & $\begin{array}{l}4883.4 \\
4716.8 \\
4501.0 \\
4284.7\end{array}$ & $\begin{array}{l}.000938 \\
.000928 \\
.000918 \\
.000908\end{array}$ \\
\hline $\begin{array}{l}62 . \\
64 . \\
66 . \\
68 . \\
70 . \\
72 . \\
74 . \\
76 . \\
78 . \\
80 .\end{array}$ & $\begin{array}{l}1.2737 \\
1.2548 \\
1.2559 \\
1.2470 \\
1.2379 \\
1.2289 \\
1.2197 \\
1.2105 \\
1.2012 \\
1.1919\end{array}$ & $\begin{array}{l}.19070 \\
.18755 \\
.18450 \\
.18153 \\
.17962 \\
.17576 \\
.17295 \\
.17017 \\
.16741 \\
.16467\end{array}$ & $\begin{array}{l}4069.2 \\
3863.8 \\
3666.3 \\
3477.4 \\
3297.6 \\
3127.1 \\
2966.0 \\
2813.8 \\
2670.5 \\
2535.6\end{array}$ & $\begin{array}{l}.000898 \\
.000890 \\
.000881 \\
.000873 \\
.000866 \\
.000858 \\
.000850 \\
.000842 \\
.000834 \\
.000826\end{array}$ & $\begin{array}{l}62 . \\
64 . \\
66 . \\
68 . \\
70 . \\
72 . \\
74 . \\
76 . \\
78 . \\
80 .\end{array}$ & $\begin{array}{l}1.2738 \\
1.2650 \\
1.2561 \\
1.2471 \\
1.2381 \\
1.2290 \\
1.2199 \\
1.2107 \\
1.2014 \\
1.1921\end{array}$ & $\begin{array}{l}.19075 \\
.18760 \\
.18455 \\
.18158 \\
.17867 \\
.17582 \\
.17301 \\
.17023 \\
.16747 \\
.16473\end{array}$ & $\begin{array}{l}4072.7 \\
3867.1 \\
3669.4 \\
3480.3 \\
3300.4 \\
3129.8 \\
2958.5 \\
2816.3 \\
2672.9 \\
2537.9\end{array}$ & $\begin{array}{l}.000898 \\
.000890 \\
.000882 \\
.000874 \\
.000865 \\
.000858 \\
.000850 \\
.000842 \\
.000834 \\
.000826\end{array}$ \\
\hline $\begin{array}{l}82 . \\
84 . \\
86 . \\
88 . \\
90 . \\
92 . \\
96 . \\
96 . \\
98 . \\
100 .\end{array}$ & $\begin{array}{l}1.1824 \\
1.1729 \\
1.1633 \\
1.1536 \\
1.1437 \\
1.1338 \\
1.1237 \\
1.1135 \\
1.1031 \\
1.0926\end{array}$ & $\begin{array}{l}.16194 \\
.159 ? 1 \\
.15649 \\
.15377 \\
.15103 \\
.14829 \\
.14554 \\
.14277 \\
.13999 \\
.13719\end{array}$ & $\begin{array}{l}2408.5 \\
2299.2 \\
2175.9 \\
2071.3 \\
1071.9 \\
1878.2 \\
1790.0 \\
1706.7 \\
1628.1 \\
1553.8\end{array}$ & $\begin{array}{l}.000818 \\
.000809 \\
.000900 \\
.000791 \\
.000781 \\
.000772 \\
.000761 \\
.000750 \\
.000739 \\
.000727\end{array}$ & $\begin{array}{l}82 . \\
84 . \\
86 . \\
88 . \\
90 . \\
92 . \\
94 . \\
96 . \\
98 . \\
100 .\end{array}$ & $\begin{array}{l}1.1826 \\
1.1731 \\
1.1635 \\
1.1538 \\
1.1440 \\
1.1340 \\
1.1239 \\
1.1137 \\
1.1034 \\
1.0929\end{array}$ & $\begin{array}{l}.16200 \\
.15928 \\
.15654 \\
.15384 \\
.15111 \\
.14837 \\
.14562 \\
.14286 \\
.14008 \\
.13728\end{array}$ & $\begin{array}{l}2410.8 \\
2291.4 \\
2179.0 \\
2073.3 \\
1973.8 \\
1880.2 \\
1791.9 \\
1708.6 \\
1629.9 \\
1555.6\end{array}$ & $\begin{array}{l}.000918 \\
.000959 \\
.000801 \\
.000791 \\
.000782 \\
.000772 \\
.000762 \\
.000751 \\
.000740 \\
.000728\end{array}$ \\
\hline $\begin{array}{l}102 . \\
104 . \\
106 . \\
108 . \\
110 . \\
112 . \\
114 . \\
116 . \\
117.843 \\
117.843 \\
118 . \\
120 .\end{array}$ & $\begin{array}{l}1.0819 \\
1.0709 \\
1.0598 \\
1.0434 \\
1.0368 \\
1.0248 \\
1.0126 \\
.0909 \\
.9979 \\
.0347 \\
.0347 \\
.0337\end{array}$ & $\begin{array}{l}.13436 \\
.13151 \\
.12864 \\
.12574 \\
.12280 \\
.11983 \\
.11582 \\
.11377 \\
.11092 \\
.01163 \\
.01164 \\
.01122\end{array}$ & $\begin{array}{r}1483.4 \\
1416.7 \\
1353.3 \\
1293.0 \\
1235.4 \\
1180.7 \\
1128.1 \\
1077.7 \\
1033.0 \\
91.5 \\
91.6 \\
93.0\end{array}$ & $\begin{array}{l}.000715 \\
.000702 \\
.000689 \\
.000675 \\
.000660 \\
.006845 \\
.000628 \\
.000611 \\
.000594 \\
.002690 \\
.002705 \\
.002893\end{array}$ & $\begin{array}{l}102 . \\
104 . \\
103 . \\
109 . \\
110 . \\
112 . \\
114 . \\
115 . \\
118 . \\
* 119.523 \\
* 119.623 \\
120 .\end{array}$ & $\begin{array}{l}1.0822 \\
1.0713 \\
1.0601 \\
1.0488 \\
1.0372 \\
1.0253 \\
1.0130 \\
1.0004 \\
.9873 \\
.9764 \\
.0385 \\
.0383\end{array}$ & $\begin{array}{l}.13446 \\
.13161 \\
.12874 \\
.12584 \\
.12291 \\
.11995 \\
.11694 \\
.11390 \\
.11081 \\
.10826 \\
.01190 \\
.01194\end{array}$ & $\begin{array}{r}1485.2 \\
1418.4 \\
1355.0 \\
1294.7 \\
1237.3 \\
1182.4 \\
1129.9 \\
1079.5 \\
1031.0 \\
992.9 \\
93.3 \\
93.6\end{array}$ & $\begin{array}{l}.000716 \\
.000703 \\
.000690 \\
.000676 \\
.000661 \\
.000645 \\
.000629 \\
.000612 \\
.000594 \\
.000579 \\
.002419 \\
.002452\end{array}$ \\
\hline $\begin{array}{l}122 . \\
124 . \\
1260^{\circ} \\
128 . \\
130 . \\
132 . \\
134 . \\
136 . \\
138 . \\
140 .\end{array}$ & $\begin{array}{l}.0329 \\
.0320 \\
.0313 \\
.0306 \\
.0299 \\
.0293 \\
.0287 \\
.0281 \\
.0276 \\
.0271\end{array}$ & $\begin{array}{l}.01200 \\
.01218 \\
.01237 \\
.01255 \\
.01273 \\
.01292 \\
.01311 \\
.01329 \\
.01348 \\
.01337\end{array}$ & $\begin{array}{r}94.5 \\
95.9 \\
97.3 \\
98.8 \\
100.2 \\
101.7 \\
103.1 \\
104.5 \\
105.9 \\
107.4\end{array}$ & $\begin{array}{l}.003081 \\
.003269 \\
.003457 \\
.003646 \\
.003835 \\
.004025 \\
.004216 \\
.004408 \\
.004601 \\
.004795\end{array}$ & 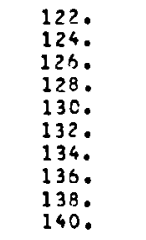 & $\begin{array}{l}.0373 \\
.0363 \\
.0354 \\
.0345 \\
.0338 \\
.0330 \\
.0323 \\
.0316 \\
.0310 \\
.0304\end{array}$ & $\begin{array}{l}.01211 \\
.01229 \\
.01247 \\
.01265 \\
.01283 \\
.01302 \\
.011320 \\
.01338 \\
.011357 \\
.011376\end{array}$ & $\begin{array}{r}95.0 \\
96.4 \\
97.8 \\
99.2 \\
100.7 \\
102.1 \\
103.5 \\
104.9 \\
106.3 \\
107.8\end{array}$ & $\begin{array}{l}.002627 \\
.002802 \\
.002975 \\
.003151 \\
.003326 \\
.003501 \\
.003677 \\
.003954 \\
.004031 \\
.004209\end{array}$ \\
\hline $\begin{array}{l}142 . \\
146 . \\
146 . \\
148 . \\
150 . \\
152 . \\
154 . \\
150^{\circ} \\
150^{\circ} \\
160 .\end{array}$ & $\begin{array}{l}.0266 \\
.0261 \\
.0255 \\
.0255 \\
.0248 \\
.0244 \\
.0240 \\
.0236 \\
.0233 \\
.0229\end{array}$ & $\begin{array}{l}.01385 \\
.01404 \\
.01423 \\
.01443 \\
.01462 \\
.01492 \\
.01502 \\
.01519 \\
.01534 \\
.01551\end{array}$ & $\begin{array}{l}108.8 \\
110.2 \\
111.6 \\
113.0 \\
114.4 \\
115.8 \\
117.2 \\
118.6 \\
120.0 \\
121.4\end{array}$ & $\begin{array}{l}.004891 \\
.005188 \\
.005387 \\
.005588 \\
.005797 \\
.005990 \\
.006210 \\
.006407 \\
.006601 \\
.006799\end{array}$ & $\begin{array}{l}142 . \\
144 . \\
145 . \\
148 . \\
150 . \\
152 . \\
154 . \\
156 . \\
158 . \\
150 .\end{array}$ & $\begin{array}{l}.0298 \\
.0293 \\
.0288 \\
.0283 \\
.0278 \\
.0273 \\
.0269 \\
.0265 \\
.0261 \\
.0257\end{array}$ & $\begin{array}{l}.01394 \\
.01413 \\
.01432 \\
.01451 \\
.01471 \\
.01491 \\
.01511 \\
.01527 \\
.011543 \\
.01550\end{array}$ & $\begin{array}{l}109.2 \\
110.6 \\
112.0 \\
113.4 \\
114.8 \\
116.2 \\
117.5 \\
118.9 \\
120.3 \\
121.7\end{array}$ & $\begin{array}{l}.004389 \\
.004570 \\
.004752 \\
.004936 \\
.005122 \\
.005311 \\
.005505 \\
.005384 \\
.005350 \\
.005039\end{array}$ \\
\hline $\begin{array}{l}165 . \\
170 . \\
175 . \\
180 . \\
185 . \\
180 . \\
105 . \\
200 . \\
210 . \\
270 .\end{array}$ & $\begin{array}{l}.0221 \\
.0214 \\
.0207 \\
.0201 \\
.0104 \\
.0188 \\
.0183 \\
.0178 \\
.0169 \\
.0161\end{array}$ & $\begin{array}{l}.01593 \\
.01635 \\
.01678 \\
.01721 \\
.01763 \\
.01805 \\
.01847 \\
.01889 \\
.01973 \\
.02052\end{array}$ & $\begin{array}{l}124.8 \\
128.2 \\
131.5 \\
134.9 \\
138.2 \\
141.5 \\
144.8 \\
148.0 \\
154.4 \\
160.7\end{array}$ & $\begin{array}{l}.007305 \\
.007824 \\
.008354 \\
.008894 \\
.000444 \\
.010004 \\
.010573 \\
.011152 \\
.012338 \\
.013562\end{array}$ & $\begin{array}{l}165 . \\
170 . \\
175 \\
180 . \\
185 . \\
100 . \\
175 \\
200 . \\
210 . \\
22.0\end{array}$ & $\begin{array}{l}.0247 \\
.0239 \\
.0231 \\
.0223 \\
.0217 \\
.0210 \\
.0204 \\
.0198 \\
.0188 \\
.0179\end{array}$ & $\begin{array}{l}.01600 \\
.01642 \\
.01644 \\
.01727 \\
.01769 \\
.01811 \\
.011853 \\
.01894 \\
.01076 \\
.02057\end{array}$ & $\begin{array}{l}125.1 \\
128.5 \\
131.9 \\
135.2 \\
136.5 \\
141.8 \\
145.0 \\
148.3 \\
154.6 \\
150.0\end{array}$ & $\begin{array}{l}.008498 \\
.006969 \\
.007450 \\
.007940 \\
.008438 \\
.008945 \\
.009440 \\
.009784 \\
.011057 \\
.011163\end{array}$ \\
\hline $\begin{array}{l}230 . \\
240 . \\
250 . \\
260 . \\
270 . \\
280 . \\
290 . \\
300 . \\
310 . \\
320 .\end{array}$ & $\begin{array}{l}.0153 \\
.0145 \\
.0140 \\
.0135 \\
.0129 \\
.0125 \\
.0122 \\
.0116 \\
.0112 \\
.0109\end{array}$ & $\begin{array}{l}.02132 \\
.02211 \\
.02285 \\
.02357 \\
.02443 \\
.02510 \\
.02595 \\
.02570 \\
.02745 \\
.0282 C\end{array}$ & $\begin{array}{l}166.8 \\
172.9 \\
178.8 \\
184.7 \\
190.5 \\
196.1 \\
201.7 \\
207.2 \\
212.6 \\
217.9\end{array}$ & $\begin{array}{l}.014824 \\
.016123 \\
.017458 \\
.018630 \\
.020238 \\
.021680 \\
.023156 \\
.024685 \\
.026207 \\
.027788\end{array}$ & $\begin{array}{l}230 . \\
240 . \\
250 . \\
260 . \\
270 . \\
280 . \\
290 . \\
390 . \\
310 . \\
320 .\end{array}$ & $\begin{array}{l}.0171 \\
.0163 \\
.0156 \\
.0150 \\
.0144 \\
.0139 \\
.0134 \\
.0129 \\
.0125 \\
.0121\end{array}$ & $\begin{array}{l}.02137 \\
.02216 \\
.02294 \\
.02372 \\
.02448 \\
.02524 \\
.02600 \\
.02675 \\
.02749 \\
.02824\end{array}$ & $\begin{array}{l}157.0 \\
173.1 \\
179.0 \\
184.9 \\
190.6 \\
195.3 \\
231.8 \\
207.3 \\
212.7 \\
219.1\end{array}$ & $\begin{array}{l}.013303 \\
.014477 \\
.015683 \\
.016922 \\
.0118192 \\
.019493 \\
.020325 \\
.022186 \\
.023578 \\
.025003\end{array}$ \\
\hline
\end{tabular}

1.0 MP. Isobar Thermal viscosity Thermal
Cond. micro- olffusivity
Wimok

Temp. Density

$g / c m * * 3$

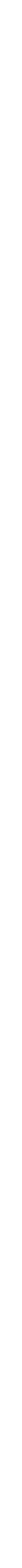

* Two phase qoundary 
Table 3. Transport Propertles of Oxygen, Isobars, SI Unlts.

$1.5 \mathrm{MP}$ Is Isober

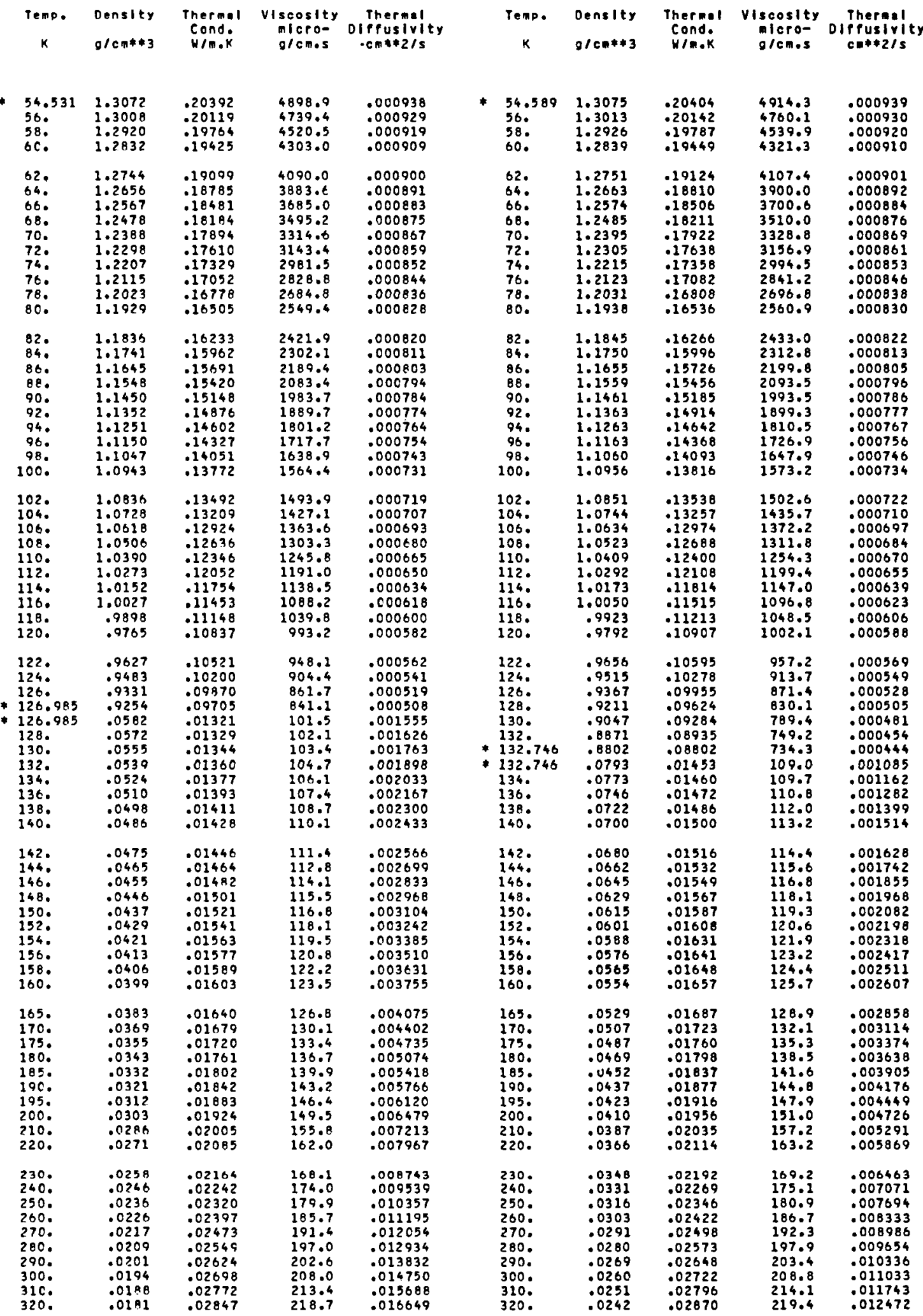

2.0 MPA Isobar

Thermal Viscosity Thernal

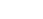


Table 5. Transoort Properties of Oxygen, Isobars, SI Units.

$2.5 \mathrm{MPa} I$ s obar

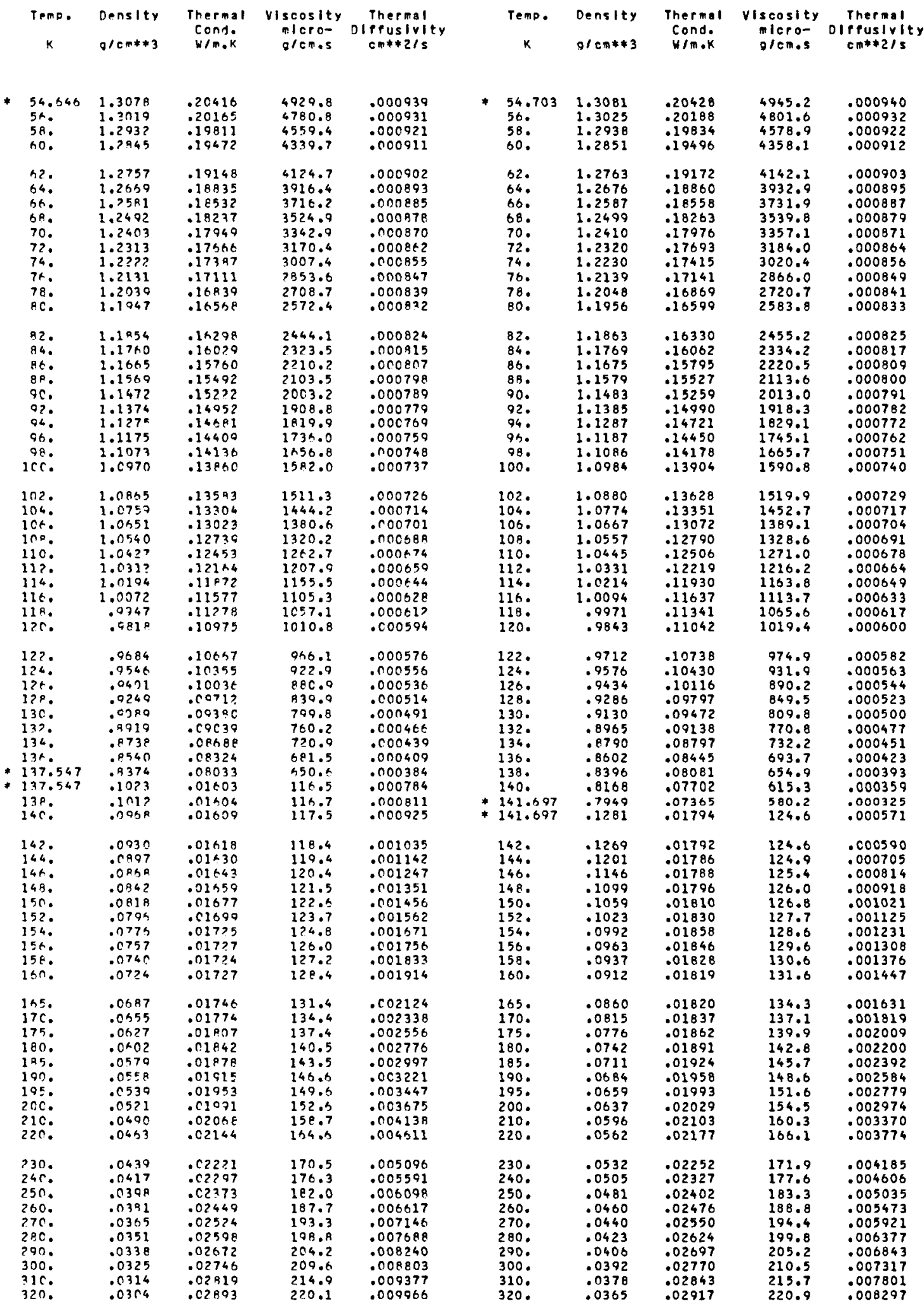

$3.0 \mathrm{MPE} 150 \mathrm{bar}$ 
Table 5. Transport Propertles of Oxyoen, Isobars, SI Units.

$3.5 \mathrm{HPa} \mathrm{I} 0 \mathrm{Obar}$

Tenp. Density
$k \quad$ g/ent*3

$\begin{array}{ll}54.760 & 1.3084 \\ 56 . & 1.3031 \\ 58 . & 1.2944 \\ 60 . & 1.2857 \\ 62 . & 1.2769\end{array}$

$\begin{array}{lll}62 . & 1.2769 & .19196 \\ 64 . & 1.2682 & .18885\end{array}$

64. 1.2682

68. 1.2506

70. 1.2417

72. $\quad 1.2327$

74. 1.2236

$\begin{array}{ll}78 . & 1.2056 \\ 80 . & 1.1964\end{array}$

$\begin{array}{ll}82 . & 1.1872 \\ 84 . & 1.1778\end{array}$

86. 1.1684

98. 1.1494

92. 1.1397

94. 1.1299

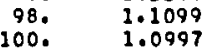

102.1 .0894

$\begin{array}{ll}102 . & 1.0894 \\ 104 . & 1.0789 \\ 106 . & 1.0582 \\ 108 . & 1.0574\end{array}$

108 : 1.0574

112.1 .0350

114. $\quad 1.0234$

$\begin{array}{rr}116 . & 1.0115 \\ 118 . & .9994\end{array}$

$\begin{array}{ll}118 . & .9994 \\ 120 . & .9868\end{array}$

$\begin{array}{ll}122 . & .9739 \\ 124 . & .9605 \\ 126 . & .9466\end{array}$

$\begin{array}{ll}126 . & .9466 \\ 128 . & .9321\end{array}$

$130 . \quad .9169$

132. $\quad .9009$

$134 . \quad .8840$

$\begin{array}{ll}136 . & .8659 \\ 138 . & .8463\end{array}$

$140 . \quad .8249$

142. .8009

142.

* 145.365

145.365

146.

148.

150.011440

$150 . \quad 136$

$154 . \quad \$ 124$

$\begin{array}{ll}156 . & 1103 \\ 150 . & 1162 \\ 160 . & .1126\end{array}$

165. .1050

$170 . \quad .0988$

$180 . \quad .0391$

$185 . \quad .0851$

$\begin{array}{ll}190 . & .0816 \\ 195 . & .0785\end{array}$

$\begin{array}{ll}200 . & .0756 \\ 210 . & .0706\end{array}$

230.0026

$230 . \quad .0326$

$\begin{array}{ll}240 . & .0594 \\ 260 . & .0565 \\ 260 . & .0539\end{array}$

$27 \mathrm{C}$.

280.

300 .

310.

.0495

.0476

.0458

.0442
.0427
Thermel VIscosity Thermal
cond. micro- Diffusivity

W/m.K g/cmos cm $\$ 2 / \mathrm{s}$

.20440

.20211

.19857

.18593

.18289

.18002

.17444

.17170

.16899

.16362

.16096

.15829

.15296

.15028

.14760

.14490

.14219
.13947

.13673

.13398
.13120

.13120

.12840

.12274

.11987

.11697

.11404

.11107

.10807

.10503

.10194

.09561

.09234

.08901

.08559

.08258

.07468

.07075

.06794

.02059
.02045

.02045
.02022

.02017

.02028

.02055

.02017

.01971

.01913

.01913

.01927

.01976

.02006

.02038

.02071

.02140

.02285

.02358

.02431

.02577

.02650

.02723

.02795

.02867
.02941
$4960.6 \quad .000941$

$4822.4 \quad .000933$

$\begin{array}{ll}4598.5 & .000923 \\ 4376.5 & .000913\end{array}$

$\begin{array}{ll}4159.5 & .000904 \\ 3949.4 & .000896\end{array}$

3949.4

3747.5

3554.7

3371.3

3197.5

3033.3
2878.4

2732.6

2595.3

2466.3

2344.9

2230.9

2123.6

1927.8

1838.4

1754.1
1674.6

1674.6

1528.5

1461.2

1336.9

1279.3

1224.5
1172.1

1122.0

1074.0

1027.9

983.6

740.7

899.2
858.9

819.6
781.0

743.0

705.4
667.7

629.5

590.1

548.5

517.5
133.8

133.4

132.9

132.8

133.6

134.2

134.9

137.8

140.3

140.3
142.8

142.8
145.5

148.2

150.9
153.7

153.7
156.5

162.2

167.

173.4
179.0

184.6

190.1

195.5
200.9

206.2

211.4
216.6

216.6
221.6
.000888

.000880

.000873

.000865

.000858

.000850

.000843
.000835

.000827

.000819

.000811

.000802

.000793

.000774

.000754
.000743

.000732

.000720

.000695

.000682

000668

.000638

.000622

.000606

.000588

.000570

.000551

.000531

.000487

.000463

.000408

.000377

.000342

.000300

.000266

.000431

.000572

.000684

.000793

.000903

.000978

.001041

.001276

.001447

.001618

.00178

.001959

.002130

.002302

.002475

.002823
.003177

.003537

.003903

.004658

.005047

.005443

.005846

.006257

.006675

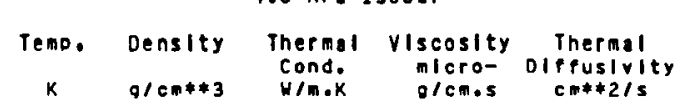


Table 5. Transport Propertles of oxypen, Isobars, SI Units.

4.5 MPE IsOber

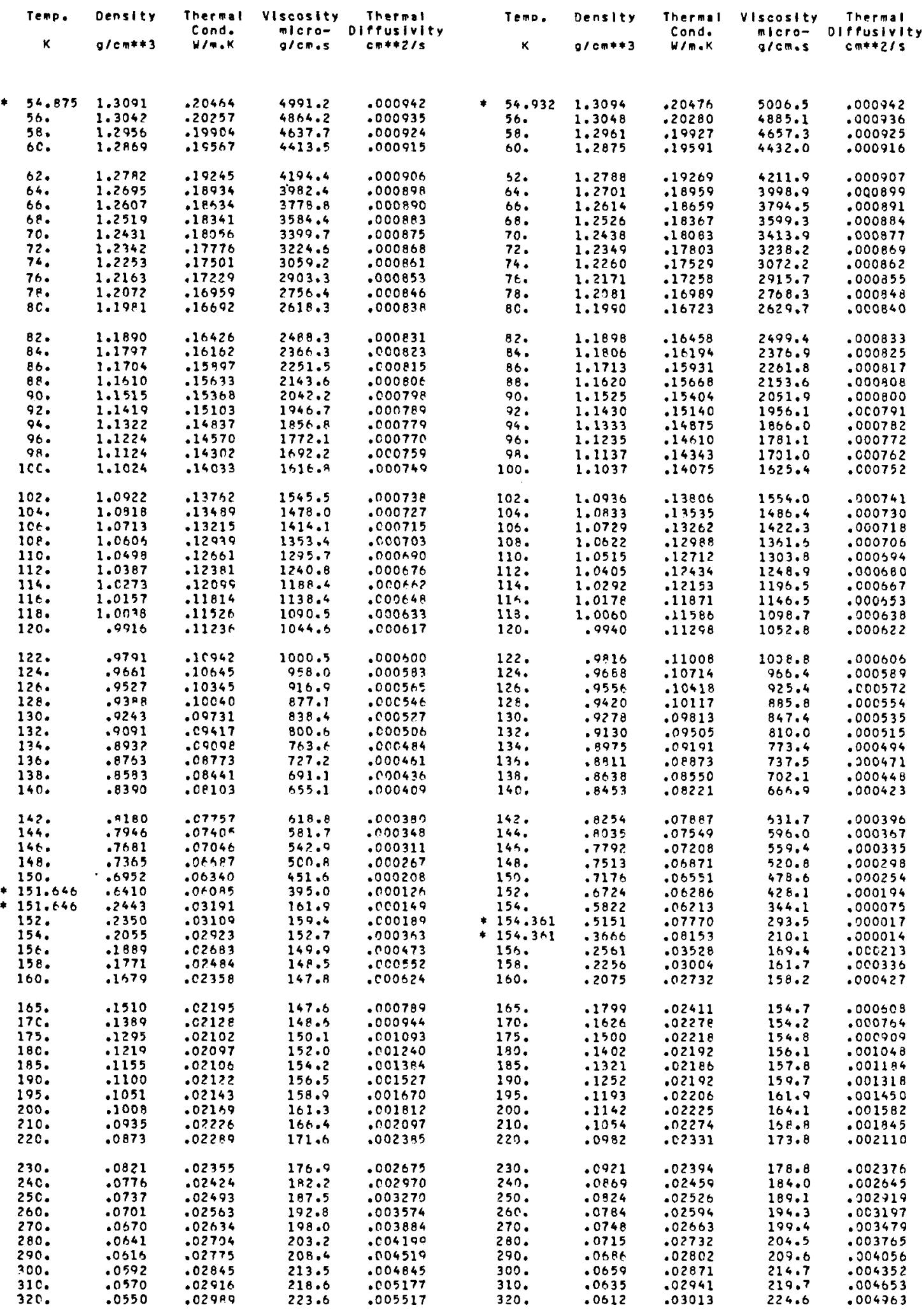

5.0 MPa Isobar

Thermal viscosity Thermal 
Table 5. Transoort Drooprties of Oxygen. Isobars, SI Units.

$5.5 \mathrm{MOA}$ Is $0 \mathrm{bar}$

\begin{tabular}{|c|c|c|c|c|c|c|c|c|c|}
\hline Tetp. & $\begin{array}{l}\text { Density } \\
0 / 0 \pi * 3\end{array}$ & $\begin{array}{l}\text { Thermal } \\
\text { Cond. } \\
\text { W/r.k }\end{array}$ & $\begin{array}{c}\text { VIscosity } \\
\text { micro- } \\
\text { g/cons }\end{array}$ & $\begin{array}{c}\text { Thermal } \\
\text { olffusivity } \\
\text { cm* } * 2 / s\end{array}$ & $\begin{array}{c}\text { Temp. } \\
k\end{array}$ & $\begin{array}{l}\text { Density } \\
3 / \mathrm{cm} * 3\end{array}$ & $\begin{array}{l}\text { Thermal } \\
\text { Cond. } \\
w / m, k\end{array}$ & $\begin{array}{c}\text { VIscosity } \\
\text { mIcro- } \\
\text { g/cmos }\end{array}$ & $\begin{array}{c}\text { Thermal } \\
\text { olfrusivity } \\
\text { co**2/s }\end{array}$ \\
\hline $\begin{array}{l}54.989 \\
54 . \\
58 . \\
6 C .\end{array}$ & $\begin{array}{l}1.3097 \\
1.3054 \\
1.2047 \\
1.2881\end{array}$ & $\begin{array}{r}.20488 \\
.20303 \\
.19950 \\
.15614\end{array}$ & $\begin{array}{l}5021.7 \\
4906.1 \\
4677.0 \\
4450.5\end{array}$ & $\begin{array}{l}.000943 \\
.000937 \\
.000926 \\
.000917\end{array}$ & $\begin{array}{l}55.046 \\
56 . \\
59 . \\
50 .\end{array}$ & $\begin{array}{l}1.3100 \\
1.3059 \\
1.2973 \\
1.2887\end{array}$ & $\begin{array}{l}.20500 \\
.20325 \\
.19973 \\
.19636\end{array}$ & $\begin{array}{l}5037.0 \\
4927.1 \\
4696.7 \\
4469.1\end{array}$ & $\begin{array}{l}.000943 \\
.000938 \\
.000927 \\
.000918\end{array}$ \\
\hline $\begin{array}{l}62 . \\
64: \\
66: \\
68 . \\
70 . \\
72 . \\
74 . \\
74: \\
78 . \\
80 .\end{array}$ & $\begin{array}{l}1.2794 \\
1.2707 \\
1.2420 \\
1.2533 \\
1.2445 \\
1.2357 \\
1.2268 \\
1.2179 \\
1.2089 \\
1.1998\end{array}$ & $\begin{array}{r}.19293 \\
.18993 \\
.18684 \\
.18393 \\
.18109 \\
.17831 \\
.17557 \\
.17287 \\
.17019 \\
.16754\end{array}$ & $\begin{array}{l}4229.4 \\
4015.5 \\
3810.2 \\
3614.3 \\
3428.1 \\
3251.7 \\
3085.1 \\
2928.1 \\
2780 . ? \\
2641.2\end{array}$ & $\begin{array}{l}.000908 \\
.000900 \\
.000893 \\
.000895 \\
.000878 \\
.000871 \\
.000864 \\
.000856 \\
.000849 \\
.000842\end{array}$ & $\begin{array}{l}52 . \\
64 . \\
66 . \\
68 . \\
70 . \\
72 . \\
74 . \\
76 . \\
78 . \\
80 .\end{array}$ & $\begin{array}{l}1.2800 \\
1.2714 \\
1.2627 \\
1.2540 \\
1.2452 \\
1.2364 \\
1.2275 \\
1.2186 \\
1.2097 \\
1.2007\end{array}$ & $\begin{array}{l}.19317 \\
.19008 \\
.18709 \\
.18419 \\
.18136 \\
.17858 \\
.17585 \\
.17315 \\
.17049 \\
.16784\end{array}$ & $\begin{array}{l}4246.9 \\
4032.1 \\
3825.9 \\
3629.2 \\
3442.3 \\
3265.3 \\
3098.1 \\
2940.5 \\
2792.1 \\
2652.6\end{array}$ & $\begin{array}{l}.000909 \\
.000901 \\
.000894 \\
.000886 \\
.000879 \\
.000872 \\
.000865 \\
.000858 \\
.000851 \\
.000843\end{array}$ \\
\hline $\begin{array}{l}82 . \\
84 . \\
86 . \\
88 . \\
90 . \\
92 . \\
96 . \\
96 . \\
98 . \\
100 .\end{array}$ & $\begin{array}{l}1.1907 \\
1.1315 \\
1.1723 \\
1.1520 \\
1.1535 \\
1.1440 \\
1.1344 \\
1.1247 \\
1.1149 \\
1.1050\end{array}$ & $\begin{array}{l}.16490 \\
.162 ? 7 \\
.15965 \\
.15702 \\
.15440 \\
.15177 \\
.14914 \\
.14649 \\
.14384 \\
.14117\end{array}$ & $\begin{array}{l}2510.4 \\
2387.5 \\
2272.1 \\
2163.5 \\
2061.5 \\
1955.5 \\
1875.5 \\
1790.1 \\
1700.0 \\
1634.0\end{array}$ & $\begin{array}{l}.000834 \\
.000827 \\
.000819 \\
.000810 \\
.000802 \\
.000793 \\
.000784 \\
.000775 \\
.000765 \\
.000755\end{array}$ & $\begin{array}{l}82 . \\
84 . \\
86 . \\
88 . \\
90 . \\
92 . \\
94 . \\
96 . \\
98 . \\
100 .\end{array}$ & $\begin{array}{l}1.1916 \\
1.1824 \\
1.1732 \\
1.1639 \\
1.1546 \\
1.1451 \\
1.1356 \\
1.1259 \\
1.1161 \\
1.1063\end{array}$ & $\begin{array}{l}.16521 \\
.16259 \\
.15998 \\
.15737 \\
.15476 \\
.15214 \\
.14951 \\
.14688 \\
.14424 \\
.14159\end{array}$ & $\begin{array}{l}2521.4 \\
2398.2 \\
2282.4 \\
2173.5 \\
2071.2 \\
1974.9 \\
1884.3 \\
1799.0 \\
1718.5 \\
1642.6\end{array}$ & $\begin{array}{l}.000836 \\
.000828 \\
.000821 \\
.000812 \\
.000804 \\
.000795 \\
.000786 \\
.000777 \\
.000767 \\
.000757\end{array}$ \\
\hline $\begin{array}{l}10 ? . \\
104 . \\
106 . \\
108 . \\
110 . \\
112 . \\
114 . \\
116 . \\
118 . \\
120 .\end{array}$ & $\begin{array}{l}1.0949 \\
1.0847 \\
1.0744 \\
1.0438 \\
1.0531 \\
1.0472 \\
1.0311 \\
1.0198 \\
1.0081 \\
.0962\end{array}$ & $\begin{array}{l}13949 \\
.12580 \\
.13309 \\
.13036 \\
.12752 \\
.12486 \\
.12208 \\
.11927 \\
.11545 \\
.11360\end{array}$ & $\begin{array}{l}1562.4 \\
1494.7 \\
1430.5 \\
1369.7 \\
1311.9 \\
1256.9 \\
1204.5 \\
1154.5 \\
1106.7 \\
1060.9\end{array}$ & $\begin{array}{l}.000744 \\
.000733 \\
.000722 \\
.000710 \\
.000697 \\
.000684 \\
.000671 \\
.000657 \\
.000643 \\
.000627\end{array}$ & $\begin{array}{l}102 . \\
104 . \\
106 . \\
108 . \\
110 . \\
112 . \\
114 . \\
116 . \\
118 . \\
120 .\end{array}$ & $\begin{array}{r}1.0963 \\
1.0861 \\
1.0758 \\
1.0654 \\
1.0548 \\
1.0440 \\
1.0330 \\
1.0217 \\
1.0102 \\
.9985\end{array}$ & $\begin{array}{l}.13892 \\
.13625 \\
.13355 \\
.13084 \\
.12812 \\
.12537 \\
.12261 \\
.11983 \\
.11703 \\
.11421\end{array}$ & $\begin{array}{l}1570.8 \\
1503.0 \\
1438.7 \\
1377.8 \\
1319.9 \\
1264.9 \\
1212.4 \\
1162.4 \\
1114.6 \\
1068.9\end{array}$ & $\begin{array}{l}.000747 \\
.000736 \\
.000725 \\
.000713 \\
.000701 \\
.000688 \\
.000675 \\
.000661 \\
.000647 \\
.000633\end{array}$ \\
\hline $\begin{array}{l}1220^{\circ} \\
1240^{\circ} \\
1260^{\circ} \\
1280^{\circ} \\
1300^{\circ} \\
1320^{\circ} \\
136^{\circ} \\
1340^{\circ} \\
1380^{\circ} \\
140^{\circ}\end{array}$ & $\begin{array}{l}.9840 \\
.9714 \\
.9595 \\
.9451 \\
.9312 \\
.9167 \\
.9016 \\
.8957 \\
.8690 \\
.8512\end{array}$ & $\begin{array}{l}.11072 \\
.10782 \\
.10489 \\
.10193 \\
.09893 \\
.09589 \\
.09282 \\
.08970 \\
.08654 \\
.08334\end{array}$ & $\begin{array}{r}1016.0 \\
974.7 \\
933.9 \\
894.5 \\
856.3 \\
819.2 \\
783.0 \\
747.5 \\
712.0 \\
378.1\end{array}$ & $\begin{array}{l}.000612 \\
.000595 \\
.000578 \\
.000551 \\
.000542 \\
.000523 \\
.000503 \\
.000482 \\
.000459 \\
.000436\end{array}$ & $\begin{array}{l}122 . \\
124 . \\
126 . \\
120^{\circ} \\
130 . \\
1320^{\circ} \\
134 . \\
136 . \\
138^{\circ} \\
140^{\circ}\end{array}$ & $\begin{array}{l}.9864 \\
.9740 \\
.9613 \\
.9481 \\
.9344 \\
.9203 \\
.9055 \\
.8901 \\
.8739 \\
.8567\end{array}$ & $\begin{array}{l}.11136 \\
.10849 \\
.10559 \\
.10266 \\
.09971 \\
.09672 \\
.09370 \\
.09064 \\
.08755 \\
.08442\end{array}$ & $\begin{array}{r}2025.0 \\
982.0 \\
942.2 \\
903.0 \\
865.0 \\
828.1 \\
792.2 \\
757.2 \\
722.8 \\
688.9\end{array}$ & $\begin{array}{l}.000617 \\
.000601 \\
.000585 \\
.000568 \\
.000550 \\
.000531 \\
.000512 \\
.000491 \\
.000470 \\
.000448\end{array}$ \\
\hline $\begin{array}{l}142 . \\
144 . \\
148 . \\
148 . \\
150 . \\
152 . \\
154 . \\
156 . \\
158 . \\
160 .\end{array}$ & $\begin{array}{l}.8322 \\
.8116 \\
.7890 \\
.7637 \\
.7344 \\
.6997 \\
.6499 \\
.5532 \\
.3267 \\
.2681\end{array}$ & $\begin{array}{l}.08010 \\
.07693 \\
.07356 \\
.07034 \\
.06731 \\
.04476 \\
.06354 \\
.06103 \\
.04513 \\
.03421\end{array}$ & $\begin{array}{l}643.8 \\
609.3 \\
574.3 \\
538.2 \\
500.0 \\
457.7 \\
406.5 \\
323.0 \\
196.7 \\
176.8\end{array}$ & $\begin{array}{l}.000411 \\
.000384 \\
.000355 \\
.000323 \\
.000287 \\
.000244 \\
.000187 \\
.000081 \\
.000117 \\
.000239\end{array}$ & $\begin{array}{l}142 . \\
1440^{\circ} \\
145 . \\
148 . \\
150 . \\
152 . \\
154 . \\
155 . \\
158 . \\
160 .\end{array}$ & $\begin{array}{l}.8385 \\
.8189 \\
.7977 \\
.7743 \\
.7481 \\
.7175 \\
.6800 \\
.6285 \\
.5380 \\
.3930\end{array}$ & $\begin{array}{l}.08127 \\
.07810 \\
.07493 \\
.07184 \\
.06891 \\
.06642 \\
.06494 \\
.06221 \\
.05811 \\
.05000\end{array}$ & $\begin{array}{l}655.3 \\
621.8 \\
588.1 \\
553.7 \\
518.2 \\
480.4 \\
438.4 \\
387.7 \\
313.7 \\
228.0\end{array}$ & $\begin{array}{l}.000424 \\
.000399 \\
.000373 \\
.000344 \\
.000314 \\
.000279 \\
.000240 \\
.000181 \\
.000102 \\
.000107\end{array}$ \\
\hline $\begin{array}{l}165 . \\
170 . \\
175 . \\
180 . \\
185 . \\
190 . \\
195 . \\
200 . \\
210 . \\
220 .\end{array}$ & $\begin{array}{l}.2156 \\
.1198 \\
.1727 \\
.1399 \\
.1497 \\
.1413 \\
.1342 \\
.1280 \\
.1177 \\
.1093\end{array}$ & $\begin{array}{l}.02712 \\
.02471 \\
.02359 \\
.02304 \\
.02278 \\
.02271 \\
.02276 \\
.02288 \\
.02326 \\
.02377\end{array}$ & $\begin{array}{l}164.5 \\
161.2 \\
160.4 \\
160.8 \\
161.9 \\
163.4 \\
165.2 \\
167.1 \\
171.5 \\
176.1\end{array}$ & $\begin{array}{l}.000451 \\
.000613 \\
.000756 \\
.000891 \\
.001021 \\
.001147 \\
.001272 \\
.001395 \\
.001641 \\
.001886\end{array}$ & $\begin{array}{l}165 . \\
170 . \\
175 . \\
190 . \\
185 . \\
190 . \\
195 . \\
200 . \\
210 . \\
220 .\end{array}$ & $\begin{array}{l}.2625 \\
.2217 \\
.1979 \\
.1813 \\
.1686 \\
.1583 \\
.1497 \\
.1424 \\
.1303 \\
.1207\end{array}$ & $\begin{array}{l}.03152 \\
.02721 \\
.02532 \\
.02435 \\
.02385 \\
.02361 \\
.02353 \\
.02356 \\
.02383 \\
.02425\end{array}$ & $\begin{array}{l}179.2 \\
170.3 \\
167.2 \\
166.4 \\
166.6 \\
167.5 \\
168.9 \\
170.5 \\
174.3 \\
178.6\end{array}$ & $\begin{array}{l}.000312 \\
.000483 \\
.000628 \\
.000760 \\
.000885 \\
.001006 \\
.001124 \\
.001241 \\
.001471 \\
.001701\end{array}$ \\
\hline $\begin{array}{l}230 . \\
240 . \\
250 . \\
260 . \\
270 . \\
280 . \\
290 . \\
300 . \\
310 . \\
320 .\end{array}$ & $\begin{array}{l}.1023 \\
.0964 \\
.0912 \\
.0967 \\
.0826 \\
.0790 \\
.0757 \\
.0727 \\
.0700 \\
.0475\end{array}$ & $\begin{array}{l}.02434 \\
.02496 \\
.02560 \\
.02628 \\
.02693 \\
.02761 \\
.02829 \\
.02897 \\
.02966 \\
.03038\end{array}$ & $\begin{array}{l}180.9 \\
185.8 \\
190.8 \\
195.8 \\
200.8 \\
205.9 \\
210.9 \\
215.8 \\
220.8 \\
225.7\end{array}$ & $\begin{array}{l}.002132 \\
.002381 \\
.002633 \\
.002889 \\
.003148 \\
.003411 \\
.003678 \\
.003949 \\
.004226 \\
.004510\end{array}$ & $\begin{array}{l}230 . \\
240 . \\
250 . \\
260 . \\
270 . \\
280 . \\
290 . \\
300 . \\
310 . \\
320 .\end{array}$ & $\begin{array}{l}.1127 \\
.1060 \\
.1001 \\
.0950 \\
.0905 \\
.0865 \\
.0828 \\
.0795 \\
.0765 \\
.0737\end{array}$ & $\begin{array}{l}.02477 \\
.02535 \\
.02596 \\
.02660 \\
.02725 \\
.02791 \\
.02857 \\
.02924 \\
.02992 \\
.03063\end{array}$ & $\begin{array}{l}183.1 \\
187.8 \\
192.6 \\
197.5 \\
202.4 \\
207.3 \\
212.2 \\
217.1 \\
221.9 \\
226.8\end{array}$ & $\begin{array}{l}.001930 \\
.002162 \\
.002396 \\
.002633 \\
.002873 \\
.003117 \\
.003364 \\
.003615 \\
.003870 \\
.004133\end{array}$ \\
\hline
\end{tabular}

* Tho Phase Roundary 
Table 5. Transport Propertles of Oxygen, Isobars, SI Units.

$6.5 \mathrm{MP} .150 \mathrm{bar}$

\begin{tabular}{|c|c|c|c|c|c|c|c|c|c|}
\hline $\begin{array}{l}\text { Temp. } \\
k\end{array}$ & $\begin{array}{l}\text { Density } \\
\text { o/cm* } * 3\end{array}$ & $\begin{array}{l}\text { Thermal } \\
\text { Cond. } \\
\text { w/m.k }\end{array}$ & $\begin{array}{c}\text { viscosity } \\
\text { mlero- } \\
\text { glemos }\end{array}$ & $\begin{array}{c}\text { Thermal } \\
\text { Diffusivity } \\
\text { cm*2/s }\end{array}$ & $\begin{array}{c}\text { Temp. } \\
k\end{array}$ & $\begin{array}{l}\text { Density } \\
0 / \mathrm{cm} * * 3\end{array}$ & $\begin{array}{l}\text { Thermel } \\
\text { Cond. } \\
\text { H/m.k }\end{array}$ & $\begin{array}{c}\text { VIscosity } \\
\text { m lero- } \\
\text { g/cmos }\end{array}$ & $\begin{array}{c}\text { Therasi } \\
\text { DIffusivity } \\
\text { com* } 2 / s\end{array}$ \\
\hline $\begin{array}{l}102 . \\
104 . \\
104 . \\
108 . \\
110 . \\
112 . \\
1140 \\
116 . \\
118 . \\
120 .\end{array}$ & $\begin{array}{l}1.0975 \\
1.0275 \\
1.0773 \\
1.0670 \\
1.0564 \\
1.0457 \\
1.0348 \\
1.0237 \\
1.0173 \\
1.0007\end{array}$ & $\begin{array}{l}.13935 \\
.13469 \\
.13401 \\
.13132 \\
.12861 \\
.12589 \\
.12314 \\
.12038 \\
.11761 \\
.11481\end{array}$ & $\begin{array}{l}1579.2 \\
1511.2 \\
1446.8 \\
1385.8 \\
1327.9 \\
1272.8 \\
1220.3 \\
1170.3 \\
1122.5 \\
1070.8\end{array}$ & $\begin{array}{l}.000750 \\
.000739 \\
.000728 \\
.000717 \\
.000705 \\
.000692 \\
.000679 \\
.000666 \\
.000652 \\
.000638\end{array}$ & $\begin{array}{l}102 . \\
104 . \\
106 . \\
108 . \\
110 . \\
112 . \\
114 . \\
116 . \\
116 . \\
120 .\end{array}$ & $\begin{array}{l}1.0989 \\
1.0889 \\
1.0788 \\
1.0685 \\
1.0580 \\
1.0474 \\
1.0366 \\
1.0256 \\
1.0143 \\
1.0028\end{array}$ & $\begin{array}{l}.13978 \\
.13713 \\
.13447 \\
.13179 \\
.12910 \\
.12639 \\
.12367 \\
.12093 \\
.11817 \\
.11540\end{array}$ & $\begin{array}{l}1587.5 \\
1519.4 \\
1454.9 \\
1393.8 \\
1335.8 \\
1280.6 \\
1228.1 \\
1178.1 \\
1130.9 \\
1084.6\end{array}$ & $\begin{array}{l}.000753 \\
.000742 \\
.000731 \\
.000720 \\
.000708 \\
.000696 \\
.000683 \\
.000670 \\
.000656 \\
.000642\end{array}$ \\
\hline $\begin{array}{l}142 . \\
1440^{\circ} \\
1460^{\circ} \\
1480^{\circ} \\
150^{\circ} \\
1520^{\circ} \\
1540^{\circ} \\
150^{\circ} \\
150^{\circ} \\
160^{\circ}\end{array}$ & $\begin{array}{l}.8444 \\
.8257 \\
.8056 \\
.7938 \\
.7597 \\
.7326 \\
.7009 \\
.6519 \\
.4095 \\
.5297\end{array}$ & $\begin{array}{l}.08238 \\
.07929 \\
.07622 \\
.0732 ? \\
.07039 \\
.06797 \\
.04679 \\
.06357 \\
.05967 \\
.05568\end{array}$ & $\begin{array}{l}656.3 \\
633.6 \\
600.9 \\
567.7 \\
534.3 \\
499.4 \\
462.2 \\
421.0 \\
372.1 \\
309.8\end{array}$ & $\begin{array}{l}.000437 \\
.000414 \\
.000380 \\
.000333 \\
.000336 \\
.000307 \\
.000277 \\
.000234 \\
.000178 \\
.000126\end{array}$ & $\begin{array}{l}142 . \\
1440^{\circ} \\
140^{\circ} \\
148 . \\
150 . \\
152 . \\
1540^{\circ} \\
155^{\circ} \\
158^{\circ} \\
150 .\end{array}$ & $\begin{array}{l}.8500 \\
.8321 \\
.8129 \\
.7924 \\
.7700 \\
.7452 \\
.7173 \\
.6847 \\
.6450 \\
.5935\end{array}$ & $\begin{array}{l}.08344 \\
.08043 \\
.07744 \\
.07452 \\
.07176 \\
.06932 \\
.06758 \\
.06489 \\
.06153 \\
.05809\end{array}$ & $\begin{array}{l}676.8 \\
644.7 \\
612.9 \\
581.1 \\
548.9 \\
516.1 \\
481.9 \\
445.6 \\
405.8 \\
360.1\end{array}$ & $\begin{array}{l}.000449 \\
.000427 \\
.000404 \\
.000380 \\
.000355 \\
.000330 \\
.000305 \\
.000270 \\
.000228 \\
.000183\end{array}$ \\
\hline $\begin{array}{l}165 . \\
170 \\
175 \\
180 \\
1850 \\
160 . \\
195 . \\
200 \\
210 \\
270\end{array}$ & $\begin{array}{l}.3235 \\
.2501 \\
.2355 \\
.2047 \\
.1887 \\
.1742 \\
.1550 \\
.1573 \\
.1433 \\
.1323\end{array}$ & $\begin{array}{l}.03808 \\
.03048 \\
.02743 \\
.02591 \\
.02508 \\
.02462 \\
.02440 \\
.02432 \\
.02444 \\
.02477\end{array}$ & $\begin{array}{l}203.8 \\
182.5 \\
175.6 \\
172.9 \\
172.0 \\
172.2 \\
173.0 \\
174.2 \\
177.4 \\
181.2\end{array}$ & $\begin{array}{l}.000205 \\
.000374 \\
.000519 \\
.000650 \\
.000771 \\
.000888 \\
.001001 \\
.0011112 \\
.001330 \\
.001545\end{array}$ & $\begin{array}{l}165 . \\
170 . \\
175 . \\
180 . \\
185 . \\
190 . \\
195 . \\
200 . \\
210 . \\
220 .\end{array}$ & $\begin{array}{l}.4127 \\
.3069 \\
.2589 \\
.2303 \\
.2103 \\
.1951 \\
.1829 \\
.1728 \\
.1566 \\
.1441\end{array}$ & $\begin{array}{l}.04563 \\
.03470 \\
.03002 \\
.02773 \\
.02648 \\
.02576 \\
.02536 \\
.02515 \\
.02510 \\
.02532\end{array}$ & $\begin{array}{l}242.8 \\
199.5 \\
186.1 \\
180.6 \\
178.2 \\
177.4 \\
177.5 \\
178.2 \\
180.7 \\
184.1\end{array}$ & $\begin{array}{l}.000163 \\
.000288 \\
.000428 \\
.000556 \\
.000675 \\
.000788 \\
.000896 \\
.001002 \\
.001209 \\
.001414\end{array}$ \\
\hline $\begin{array}{l}230 . \\
240 . \\
250 . \\
250 . \\
270 . \\
280 . \\
290 . \\
300 . \\
310 . \\
320 .\end{array}$ & $\begin{array}{l}.1233 \\
.1157 \\
.1092 \\
.1035 \\
.0995 \\
.0940 \\
.0900 \\
.0863 \\
.0830 \\
.0800\end{array}$ & $\begin{array}{l}.02523 \\
.02575 \\
.02633 \\
.02694 \\
.02757 \\
.02821 \\
.02886 \\
.02951 \\
.03018 \\
.03088\end{array}$ & $\begin{array}{l}185.5 \\
189.9 \\
194.5 \\
199.2 \\
204.0 \\
208.8 \\
213.6 \\
218.4 \\
223.2 \\
227.9\end{array}$ & $\begin{array}{l}.001761 \\
.001978 \\
.002197 \\
.002418 \\
.002642 \\
.002869 \\
.003099 \\
.003332 \\
.003570 \\
.003815\end{array}$ & $\begin{array}{l}230 . \\
240 . \\
250 . \\
260 . \\
270 . \\
260 . \\
290 . \\
300 . \\
310 . \\
320 .\end{array}$ & $\begin{array}{l}.1340 \\
.1255 \\
.1183 \\
.1120 \\
.1065 \\
.1016 \\
.0972 \\
.0932 \\
.0895 \\
.0162\end{array}$ & $\begin{array}{l}.02570 \\
.02618 \\
.02672 \\
.02729 \\
.02789 \\
.02851 \\
.02915 \\
.02979 \\
.03044 \\
.03114\end{array}$ & $\begin{array}{l}187.9 \\
192.1 \\
196.5 \\
201.1 \\
205.7 \\
210.3 \\
215.0 \\
219.7 \\
224.4 \\
229.1\end{array}$ & $\begin{array}{l}.001617 \\
.001821 \\
.002027 \\
.002235 \\
.002445 \\
.002657 \\
.002873 \\
.003091 \\
.003313 \\
.003542\end{array}$ \\
\hline
\end{tabular}

7.0 MP. Isober

Thermel Viscosity Thereal 
Tahle 5. Transoort Properties of Dxygen, Isobars, SI Units.

7.5 MP. IsOtar

B.0 MPa Isobar

\begin{tabular}{|c|c|c|c|c|c|c|c|c|c|}
\hline $\begin{array}{c}\text { Temp. } \\
k\end{array}$ & $\begin{array}{l}\text { Density } \\
\text { g/cm**3 }\end{array}$ & $\begin{array}{l}\text { Thermal } \\
\text { Cond. } \\
\text { w/mok }\end{array}$ & $\begin{array}{c}\text { Viscosity } \\
\text { micro- } \\
\text { g/cmes }\end{array}$ & $\begin{array}{c}\text { Thermal } \\
\text { Diffusivity } \\
\text { co* } 2 / s\end{array}$ & $\begin{array}{c}\text { Teno. } \\
k\end{array}$ & $\begin{array}{l}\text { Denslty } \\
\text { g/cm**3 }\end{array}$ & $\begin{array}{l}\text { Thermal } \\
\text { Cond. } \\
\text { w/mok }\end{array}$ & $\begin{array}{c}\text { Viscosity } \\
\text { micro- } \\
\text { g/cmos }\end{array}$ & $\begin{array}{c}\text { Theemal } \\
\text { Diffusivity } \\
\text { c m**2/s }\end{array}$ \\
\hline $\begin{array}{l}55.216 \\
56 . \\
58 . \\
60 .\end{array}$ & $\begin{array}{l}1.3110 \\
1.3076 \\
1.2990 \\
1.2904\end{array}$ & $\begin{array}{l}.20537 \\
.20393 \\
.20042 \\
.14708\end{array}$ & $\begin{array}{l}5082.4 \\
4990.4 \\
4756.1 \\
4524.9\end{array}$ & $\begin{array}{l}.000945 \\
.000940 \\
.000930 \\
.000921\end{array}$ & $\begin{array}{l}\text { * } 55.273 \\
56 . \\
58 . \\
\text { no. }\end{array}$ & $\begin{array}{l}1.3113 \\
1.3082 \\
1.2996 \\
1.2910\end{array}$ & $\begin{array}{l}.20549 \\
.20416 \\
.20065 \\
.19731\end{array}$ & $\begin{array}{l}5097.4 \\
5011.6 \\
4776.0 \\
4543.6\end{array}$ & $\begin{array}{l}.000945 \\
.000941 \\
.000931 \\
.000922\end{array}$ \\
\hline $\begin{array}{l}62 . \\
64 . \\
66: \\
68: \\
70 . \\
72 . \\
74 . \\
76 . \\
78 . \\
86 .\end{array}$ & $\begin{array}{l}1.2819 \\
1.2733 \\
1.2648 \\
1.2560 \\
1.2473 \\
1.2385 \\
1.2298 \\
1.2210 \\
1.2121 \\
1.2032\end{array}$ & $\begin{array}{l}.19388 \\
.19081 \\
.18784 \\
.18496 \\
.18215 \\
.17939 \\
.17668 \\
.17401 \\
.17137 \\
.16976\end{array}$ & $\begin{array}{l}4299.5 \\
4081.9 \\
3873.1 \\
3674.0 \\
3484.9 \\
3306.0 \\
3137.0 \\
2977.7 \\
2827.8 \\
2686.0\end{array}$ & $\begin{array}{l}.000912 \\
.000905 \\
.000897 \\
.000890 \\
.000883 \\
.000876 \\
.000869 \\
.000862 \\
.000855 \\
.000848\end{array}$ & $\begin{array}{l}32 . \\
64 . \\
36 . \\
68 . \\
70 . \\
72 . \\
74 . \\
76 . \\
78 . \\
90 .\end{array}$ & $\begin{array}{l}1.2825 \\
1.2739 \\
1.2653 \\
1.2566 \\
1.2480 \\
1.2393 \\
1.2305 \\
1.2217 \\
1.2229 \\
1.2040\end{array}$ & $\begin{array}{l}.19412 \\
.19105 \\
.18800 \\
.18521 \\
.18241 \\
.17966 \\
.17696 \\
.17430 \\
.17167 \\
.16906\end{array}$ & $\begin{array}{l}4317.1 \\
4098.5 \\
3888.9 \\
3589.0 \\
3499.2 \\
3319.5 \\
3149.9 \\
2990.1 \\
2839.7 \\
2698.3\end{array}$ & $\begin{array}{l}.000914 \\
.000708 \\
.000898 \\
.000891 \\
.000884 \\
.000877 \\
.000871 \\
.000864 \\
.000857 \\
.000950\end{array}$ \\
\hline $\begin{array}{l}82 . \\
84 . \\
86 . \\
88 . \\
90 . \\
92 . \\
94 . \\
96 . \\
98 . \\
100 .\end{array}$ & $\begin{array}{l}1.1942 \\
1.1851 \\
1.1760 \\
1.1569 \\
1.1576 \\
1.1483 \\
1.1389 \\
1.1294 \\
1.1198 \\
1.1100\end{array}$ & $\begin{array}{l}.16615 \\
.16356 \\
.16 C 98 \\
.1584 C \\
.15591 \\
.15323 \\
.15064 \\
.14804 \\
.14544 \\
.14283\end{array}$ & $\begin{array}{l}2554.4 \\
2430.0 \\
2313.1 \\
2203.3 \\
2100.0 \\
2003.0 \\
1911.6 \\
1825.6 \\
1744.5 \\
1668.1\end{array}$ & $\begin{array}{l}.000841 \\
.000834 \\
.000826 \\
.000818 \\
.000810 \\
.000802 \\
.000793 \\
.000784 \\
.000775 \\
.000765\end{array}$ & $\begin{array}{l}82 . \\
84 . \\
86 . \\
88 . \\
90 . \\
92 . \\
94 . \\
96 . \\
98 . \\
100 .\end{array}$ & $\begin{array}{l}1.1950 \\
1.1860 \\
1.1770 \\
1.1678 \\
1.1586 \\
1.1493 \\
1.1399 \\
1.1305 \\
1.1209 \\
1.1113\end{array}$ & $\begin{array}{l}.16646 \\
.16388 \\
.16131 \\
.15874 \\
.15616 \\
.15359 \\
.15101 \\
.14843 \\
.14584 \\
.14323\end{array}$ & $\begin{array}{l}2565.4 \\
2440.6 \\
2323.3 \\
2213.2 \\
2109.6 \\
2012.3 \\
1920.7 \\
1834.4 \\
1753.2 \\
1676.5\end{array}$ & $\begin{array}{l}.000843 \\
.000836 \\
.000828 \\
.000820 \\
.000812 \\
.000804 \\
.000796 \\
.000787 \\
.000778 \\
.000768\end{array}$ \\
\hline $\begin{array}{l}102 . \\
104 . \\
100 \\
108 \\
110 . \\
112 . \\
114 . \\
116 . \\
118 . \\
120 .\end{array}$ & $\begin{array}{l}1.1002 \\
1.0903 \\
1.0802 \\
1.0700 \\
1.0596 \\
1.0491 \\
1.0384 \\
1.0275 \\
1.0163 \\
1.0050\end{array}$ & $\begin{array}{l}.14020 \\
.13757 \\
.13492 \\
.13226 \\
.12958 \\
.12689 \\
.12419 \\
.12147 \\
.111873 \\
.11598\end{array}$ & $\begin{array}{l}1595.8 \\
1527.5 \\
1463.0 \\
1401.7 \\
1343.6 \\
1288.4 \\
1235.9 \\
1185.8 \\
1138.1 \\
1092.4\end{array}$ & $\begin{array}{l}.000755 \\
.000745 \\
.000734 \\
.000723 \\
.000712 \\
.000700 \\
.000687 \\
.000674 \\
.000661 \\
.000347\end{array}$ & $\begin{array}{l}102 . \\
104 . \\
106 . \\
108 . \\
110 . \\
112 . \\
114 . \\
113 . \\
118 . \\
120 .\end{array}$ & $\begin{array}{l}1.1015 \\
1.0916 \\
1.0816 \\
1.0715 \\
1.0612 \\
1.0508 \\
1.0401 \\
1.0293 \\
1.0183 \\
1.0070\end{array}$ & $\begin{array}{l}.14062 \\
.13800 \\
.13537 \\
.13272 \\
.13006 \\
.12739 \\
.12470 \\
.12200 \\
.111929 \\
.11656\end{array}$ & $\begin{array}{l}1604.1 \\
1535.7 \\
1471.0 \\
1409.6 \\
1351.4 \\
1296.2 \\
1243.6 \\
1193.5 \\
1145.7 \\
1100.1\end{array}$ & $\begin{array}{l}.000758 \\
.000743 \\
.000737 \\
.000726 \\
.000715 \\
.000703 \\
.000691 \\
.000678 \\
.000665 \\
.000652\end{array}$ \\
\hline $\begin{array}{l}122 . \\
124 . \\
126 . \\
120^{\circ} \\
130 . \\
132 . \\
134 . \\
136 . \\
138 . \\
140 .\end{array}$ & $\begin{array}{l}.9933 \\
.9914 \\
.9692 \\
.9566 \\
.9437 \\
.9303 \\
.9165 \\
.9022 \\
.9872 \\
.8716\end{array}$ & $\begin{array}{l}.11321 \\
.11042 \\
.10762 \\
.10479 \\
.10195 \\
.09908 \\
.09619 \\
.09328 \\
.09036 \\
.08741\end{array}$ & $\begin{array}{r}1048.7 \\
1006.7 \\
984.4 \\
927.6 \\
890.1 \\
853.9 \\
818.7 \\
784.6 \\
751.3 \\
718.8\end{array}$ & $\begin{array}{l}.000633 \\
.000618 \\
.000603 \\
.000587 \\
.000570 \\
.000553 \\
.000536 \\
.000518 \\
.000499 \\
.000480\end{array}$ & $\begin{array}{l}122 . \\
124 . \\
126 . \\
128 . \\
130 . \\
132 . \\
134 . \\
135 . \\
139 . \\
140 .\end{array}$ & $\begin{array}{r}.9955 \\
.9838 \\
.9717 \\
.9593 \\
.9466 \\
.9335 \\
.9199 \\
.9059 \\
.8913 \\
.8761\end{array}$ & $\begin{array}{l}.11381 \\
.11105 \\
.10827 \\
.10547 \\
.10266 \\
.09983 \\
.09698 \\
.09412 \\
.09124 \\
.08835\end{array}$ & $\begin{array}{r}1056.4 \\
1014.5 \\
974.2 \\
935.5 \\
898.2 \\
862.1 \\
827.2 \\
793.3 \\
760.3 \\
728.1\end{array}$ & $\begin{array}{l}.000538 \\
.000623 \\
.000608 \\
.000593 \\
.000577 \\
.000560 \\
.000543 \\
.000526 \\
.000508 \\
.000489\end{array}$ \\
\hline $\begin{array}{l}142 . \\
144 . \\
146 . \\
148 . \\
150 . \\
1520^{\circ} \\
154 . \\
156 . \\
158 . \\
160 .\end{array}$ & $\begin{array}{l}.8552 \\
.8380 \\
.8197 \\
.8002 \\
.7791 \\
.7562 \\
.7309 \\
.7024 \\
.6693 \\
.6298\end{array}$ & $\begin{array}{l}.08447 \\
.08152 \\
.07860 \\
.07575 \\
.07305 \\
.07053 \\
.06881 \\
.06616 \\
.06296 \\
.05970\end{array}$ & $\begin{array}{l}686.9 \\
655.4 \\
624.3 \\
593.4 \\
562.4 \\
531.1 \\
499.0 \\
465.8 \\
430.7 \\
392.7\end{array}$ & $\begin{array}{l}.000460 \\
.000439 \\
.000417 \\
.000395 \\
.000373 \\
.000350 \\
.000328 \\
.000299 \\
.000264 \\
.000228\end{array}$ & $\begin{array}{l}142 . \\
1440^{\circ} \\
146 . \\
148 . \\
150^{\circ} \\
150^{\circ} \\
1540^{\circ} \\
156^{\circ} \\
158^{\circ} \\
160^{\circ}\end{array}$ & $\begin{array}{l}.8503 \\
.8436 \\
.8260 \\
.8074 \\
.7875 \\
.7660 \\
.7427 \\
.7169 \\
.6880 \\
.6548\end{array}$ & $\begin{array}{l}.08545 \\
.08257 \\
.07971 \\
.07692 \\
.07427 \\
.07187 \\
.07000 \\
.06738 \\
.06431 \\
.06129\end{array}$ & $\begin{array}{l}696.6 \\
665.7 \\
635.2 \\
605.0 \\
574.9 \\
544.8 \\
514.3 \\
483.3 \\
451.2 \\
417.5\end{array}$ & $\begin{array}{l}.000470 \\
.000450 \\
.000430 \\
.000409 \\
.000387 \\
.000367 \\
.000348 \\
.000322 \\
.000293 \\
.000262\end{array}$ \\
\hline $\begin{array}{l}165 . \\
170 \\
1750 \\
180 \\
185 \\
190 . \\
195 . \\
200 . \\
210 . \\
220 .\end{array}$ & $\begin{array}{l}.4891 \\
.3618 \\
.2958 \\
.2584 \\
.2335 \\
.2151 \\
.2006 \\
.1888 \\
.1703 \\
.1562\end{array}$ & $\begin{array}{l}.05072 \\
.03963 \\
.03311 \\
.02986 \\
.02808 \\
.02704 \\
.02642 \\
.02606 \\
.02580 \\
.02591\end{array}$ & $\begin{array}{l}287.2 \\
222.6 \\
199.4 \\
189.9 \\
185.4 \\
183.3 \\
182.6 \\
182.7 \\
184.3 \\
187.1\end{array}$ & $\begin{array}{l}.000170 \\
.000236 \\
.000356 \\
.000478 \\
.000593 \\
.000702 \\
.000807 \\
.000909 \\
.001107 \\
.001301\end{array}$ & $\begin{array}{l}165 . \\
170 . \\
175 . \\
180 . \\
185 . \\
190 . \\
195 . \\
200 . \\
210 . \\
220 .\end{array}$ & $\begin{array}{l}.5444 \\
.4190 \\
.3367 \\
.2890 \\
.2582 \\
.2361 \\
.2192 \\
.2055 \\
.1844 \\
.1685\end{array}$ & $\begin{array}{l}.05358 \\
.04428 \\
.03660 \\
.03229 \\
.02988 \\
.02845 \\
.02758 \\
.02704 \\
.02656 \\
.02653\end{array}$ & $\begin{array}{l}325.7 \\
250.8 \\
215.9 \\
200.9 \\
193.8 \\
190.0 \\
188.2 \\
187.5 \\
188.2 \\
190.4\end{array}$ & $\begin{array}{l}.000196 \\
.0002117 \\
.000305 \\
.000416 \\
.000525 \\
.000630 \\
.000731 \\
.000828 \\
.001018 \\
.001203\end{array}$ \\
\hline $\begin{array}{l}230 . \\
240 . \\
250 . \\
260 . \\
270 . \\
280 . \\
290 . \\
300 . \\
310 . \\
320 .\end{array}$ & $\begin{array}{l}.1440 \\
.1355 \\
.1275 \\
.1206 \\
.1145 \\
.1092 \\
.1044 \\
.1000 \\
.0961 \\
.0925\end{array}$ & $\begin{array}{l}.02620 \\
.02662 \\
.02711 \\
.02756 \\
.02823 \\
.02883 \\
.02944 \\
.03006 \\
.03071 \\
.03140\end{array}$ & $\begin{array}{l}190.6 \\
194.5 \\
198.6 \\
203.0 \\
207.4 \\
211.9 \\
216.5 \\
221.1 \\
225.7 \\
230.3\end{array}$ & $\begin{array}{l}.001494 \\
.001687 \\
.001881 \\
.002077 \\
.002274 \\
.002475 \\
.002677 \\
.002882 \\
.003091 \\
.003307\end{array}$ & $\begin{array}{l}230 . \\
240 . \\
250 . \\
260 . \\
270 . \\
280 . \\
290 . \\
300 . \\
310 . \\
320 .\end{array}$ & $\begin{array}{l}.1559 \\
.1455 \\
.1368 \\
.1292 \\
.1226 \\
.1168 \\
.1116 \\
.1069 \\
.1027 \\
.0988\end{array}$ & $\begin{array}{l}.02673 \\
.02708 \\
.02753 \\
.02803 \\
.02858 \\
.02915 \\
.02974 \\
.03035 \\
.03098 \\
.03166\end{array}$ & $\begin{array}{l}193.4 \\
197.0 \\
200.9 \\
205.0 \\
209.2 \\
213.6 \\
218.1 \\
222.6 \\
227.1 \\
231.6\end{array}$ & $\begin{array}{l}.001387 \\
.001570 \\
.001754 \\
.001939 \\
.002126 \\
.002316 \\
.002507 \\
.002701 \\
.002898 \\
.003102\end{array}$ \\
\hline
\end{tabular}

* Tro Phase Boundary 
Table 5. Transport Propertles of Oxyoen, Isobars, SI Units.

B.5 MPa Isobar

\begin{tabular}{|c|c|c|}
\hline $\begin{array}{c}\text { Teno. } \\
K\end{array}$ & $\begin{array}{l}\text { Density } \\
\text { g/cm } * 3\end{array}$ & $\begin{array}{l}\text { Thernol } \\
\text { Cond. } \\
\text { H/m.k }\end{array}$ \\
\hline $\begin{array}{l}55.330 \\
56 . \\
58 . \\
60 .\end{array}$ & $\begin{array}{l}1.3116 \\
1.3087 \\
1.3002 \\
1.2916\end{array}$ & $\begin{array}{l}.20561 \\
.20439 \\
.20088 \\
.19754\end{array}$ \\
\hline $\begin{array}{l}62 . \\
64 . \\
66 . \\
68 . \\
70 . \\
72 . \\
74 . \\
76 . \\
78 . \\
80 .\end{array}$ & $\begin{array}{l}1.2831 \\
1.2745 \\
1.2659 \\
1.2573 \\
1.2486 \\
1.2400 \\
1.2312 \\
1.2225 \\
1.2137 \\
1.2048\end{array}$ & $\begin{array}{l}.19436 \\
.19130 \\
.18834 \\
.18547 \\
.18267 \\
.17993 \\
.17724 \\
.17458 \\
.17196 \\
.16936\end{array}$ \\
\hline $\begin{array}{l}82 . \\
84 . \\
86 . \\
88 . \\
90 \\
92 . \\
94 . \\
96 . \\
98 . \\
100 .\end{array}$ & $\begin{array}{l}1.1959 \\
1.1869 \\
1.1770 \\
1.1688 \\
1.1590 \\
1.1504 \\
1.1410 \\
1.1316 \\
1.1221 \\
1.1125\end{array}$ & $\begin{array}{l}.16677 \\
.16420 \\
.16164 \\
.15907 \\
.15651 \\
.15395 \\
.15138 \\
.14881 \\
.14623 \\
.14364\end{array}$ \\
\hline $\begin{array}{l}102 . \\
104 . \\
106 . \\
108 . \\
110 . \\
112 . \\
114 . \\
116 . \\
118 . \\
120 .\end{array}$ & $\begin{array}{l}1.1028 \\
1.0930 \\
1.0830 \\
1.0730 \\
1.0628 \\
1.0524 \\
1.0418 \\
1.0311 \\
1.0202 \\
1.0091\end{array}$ & $\begin{array}{l}.14104 \\
.13843 \\
.13581 \\
.13318 \\
.13054 \\
.12788 \\
.12521 \\
.12253 \\
.11984 \\
.11713\end{array}$ \\
\hline $\begin{array}{l}1220^{\circ} \\
1240^{\circ} \\
1260^{\circ} \\
120^{\circ} \\
130^{\circ} \\
1320^{\circ} \\
1360^{\circ} \\
1360^{\circ} \\
1380^{\circ} \\
140^{\circ}\end{array}$ & $\begin{array}{l}.9977 \\
.9961 \\
.9742 \\
.9520 \\
.9495 \\
.9366 \\
.9233 \\
.9095 \\
.8953 \\
.0805\end{array}$ & $\begin{array}{l}.11440 \\
.11167 \\
.10891 \\
.10615 \\
.10336 \\
.10057 \\
.09775 \\
.09493 \\
.09209 \\
.08925\end{array}$ \\
\hline $\begin{array}{l}142 . \\
144 . \\
140^{\circ} \\
148 . \\
150 . \\
152 . \\
154 . \\
150^{\circ} \\
158 . \\
160 .\end{array}$ & $\begin{array}{l}.8350 \\
.8489 \\
.8319 \\
.8141 \\
.7951 \\
.7749 \\
.7531 \\
.7294 \\
.7034 \\
.6743\end{array}$ & $\begin{array}{l}.08640 \\
.08357 \\
.08077 \\
.07804 \\
.07543 \\
.07305 \\
.07114 \\
.06856 \\
.06559 \\
.06269\end{array}$ \\
\hline $\begin{array}{l}165 . \\
170 . \\
175 . \\
180 . \\
185 . \\
190 . \\
195 . \\
200 . \\
210 . \\
220 .\end{array}$ & $\begin{array}{l}.5331 \\
.4707 \\
.3797 \\
.3219 \\
.2846 \\
.2583 \\
.2384 \\
.2727 \\
.1988 \\
.1810\end{array}$ & $\begin{array}{l}.05557 \\
.04795 \\
.04017 \\
.03496 \\
.03197 \\
.03001 \\
.02885 \\
.02811 \\
.02737 \\
.02718\end{array}$ \\
\hline $\begin{array}{l}230 . \\
240 . \\
250 . \\
260 . \\
270 . \\
280 . \\
290 . \\
300 . \\
310 . \\
320 .\end{array}$ & $\begin{array}{l}.1671 \\
.1557 \\
.1461 \\
.1379 \\
.1308 \\
.1265 \\
.1189 \\
.1138 \\
.1992 \\
.1051\end{array}$ & $\begin{array}{l}.02728 \\
.02756 \\
.02795 \\
.02842 \\
.02893 \\
.02948 \\
.03605 \\
.03064 \\
.03125 \\
.03152\end{array}$ \\
\hline
\end{tabular}

9.0 MPa I sobar

$\begin{array}{ll}55.386 & 1.3119 \\ 50 . & 1.3093 \\ 58 . & 1.3008 \\ 60 . & 1.2922 \\ 62 . & 1.2837 \\ 64 . & 1.2751 \\ 65 . & 1.2665 \\ 68 . & 1.2579 \\ 70 . & 1.2493 \\ 72 . & 1.2407 \\ 74 . & 1.2320 \\ 76 . & 1.2232 \\ 78 . & 1.2144 \\ 80 . & 1.2056\end{array}$

$\begin{array}{lll}.20573 & 5127.5 & .000946 \\ .20461 & 5054.1 & .000943 \\ .20111 & 4815.0 & .000933 \\ .19778 & 4581.0 & .000924 \\ .19459 & 4352.4 & .000916 \\ .19154 & 4131.8 & .000908 \\ .16859 & 3920.4 & .000901 \\ .18572 & 3718.9 & .000894 \\ .18293 & 3527.6 & .000887 \\ .18020 & 3346.7 & .000880 \\ .17751 & 3175.8 & .000873 \\ .17487 & 3014.9 & .000967 \\ .17225 & 2863.5 & .000850 \\ .16966 & 2721.1 & .000853\end{array}$

\subsection{7}

$$
\begin{aligned}
& 1.1967 \\
& 1.1878
\end{aligned}
$$

$$
1.1788
$$

1.1697 1.1606 1.1514
1.1421 1.1421
1.1327 1.1327
1.1233
1.1137

.16708

.16708
.16452

.16196

.15941

.15686

.15431

.15175

.14019
.14662

.14662
.14404

$1.1041 \quad .14146$ 1.0943 1.0844 1.0744 1.0744 1.0540 1.0436 1.0329 1.0221

.13886 .13625

.13364

.13101

.12837
.12572

.12572

.12038
.11769

.11499

$\begin{array}{ll}122 . & .9999 \\ 124 . & .9884\end{array}$

$\begin{array}{ll}124 . & .9884 \\ 125 . & .9768\end{array}$

$128 . \quad .9646$

130.
132.

$134 . \quad .9265$

.9265

.8991

.11499
.11227

.10955

.10681

.10129

.09851

.09572

.09292

.8698
.8539

.8375

.08733

.08454

.08179

.07911

.07654

.0741

.07224

.06970

.06401

2587.3

2461.7

2363.7

2232.9

2128.7

2030.8

1938.7

1852.0
1770.3

1693.3

.000846

.000339

.000832

.000324

.000816

.00080

.000800

.000791

$1320.6 \quad .000764$

$1551.9 \quad .000754$

$1486.9 \quad .000743$

$1425.3 \quad .00073$

$1367.0 \quad .000722$

$1311.5 \quad .000710$

$1258.9 \quad .00069$

.000686

$\begin{array}{ll}1160.9 & .000674 \\ 1115.2 & .000661\end{array}$

$1071.6 \quad .000647$

$1029.7 \quad .000633$

$989.5 \quad .00051$

$951.1 \quad .000604$

$914.0 \quad .00058$

$\begin{array}{ll}879.2 & .000574 \\ 843.6 & .000558\end{array}$

810.1 .00054

$\begin{array}{ll}777.5 & .000524 \\ 745.0 & .000507\end{array}$

715.2 .000489

$685.1 \quad .00047$

355.6 .000453

$626.5 \quad .000434$

$597.9 \quad .000416$

$559.5 \quad .000397$

$541.2 \quad .00033$

$\begin{array}{ll}513.0 & .000360 \\ 484.5 & .000336\end{array}$

$\begin{array}{ll}454.6 & .000336 \\ & .000312\end{array}$

.05723
.05045

.05045

$381.0 \quad .000255$

$308.5 \quad .000231$

$256.5 \quad .000264$

$228.6 \quad .000335$

$\begin{array}{ll}213.8 & .000424 \\ 205.7 & .000517\end{array}$

$201.1 \quad .000010$

$198.6 \quad .000700$

$196.8 \quad .300874$

.03022

.02823

.02135

.02786

$199.5 \quad .001212$

.1784

.1660

.1555

.1467

.02806

.02840

$199.5 \quad .001212$

$202.3 \quad .001378$

$\begin{array}{ll}205.6 & .001545 \\ 209 . ? & .001713\end{array}$

$209 . ? \quad .001713$

$\begin{array}{ll}213.1 & .001882 \\ 217.2 & .002053\end{array}$

$\begin{array}{ll}217.2 & .002053 \\ 221.4 & .002225\end{array}$

$\begin{array}{ll}221.4 & .002225 \\ 225.6 & .00239\end{array}$

$229.9 \quad .00257$

$\begin{array}{ll}229.9 & .002577 \\ 234.3 & .002751\end{array}$ 


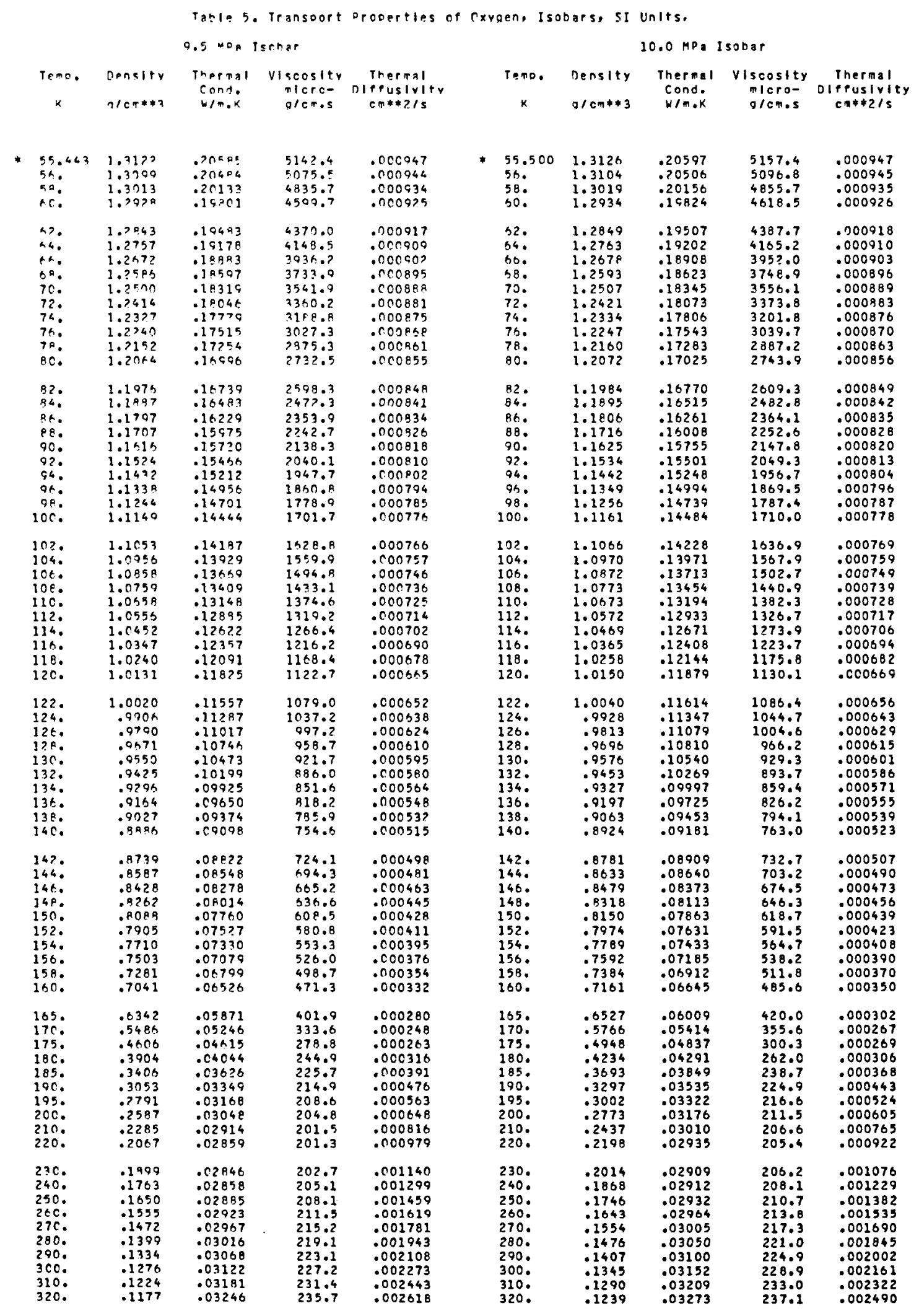


Tacle 5. Transport properties of exygen, Isobars, SI Units.

11. C MP. Isobar

$12.0 \mathrm{MPa}$ I sobar

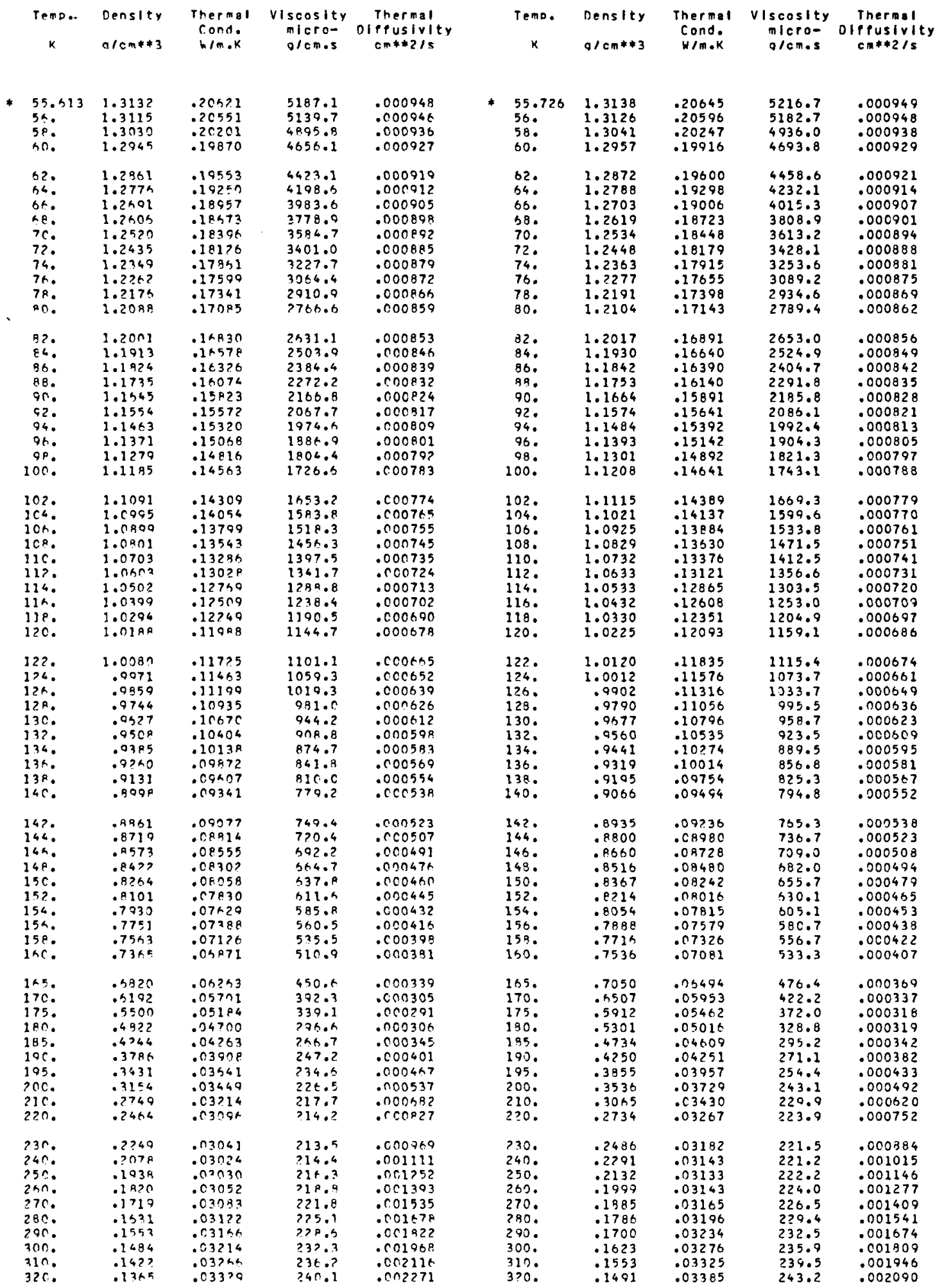

* Tro prase pounctary 
Tatile 5. Iransoort Pronerties of Exyoen. Isobars, SI Unlts.

$13.0 M P$ Isot:ar

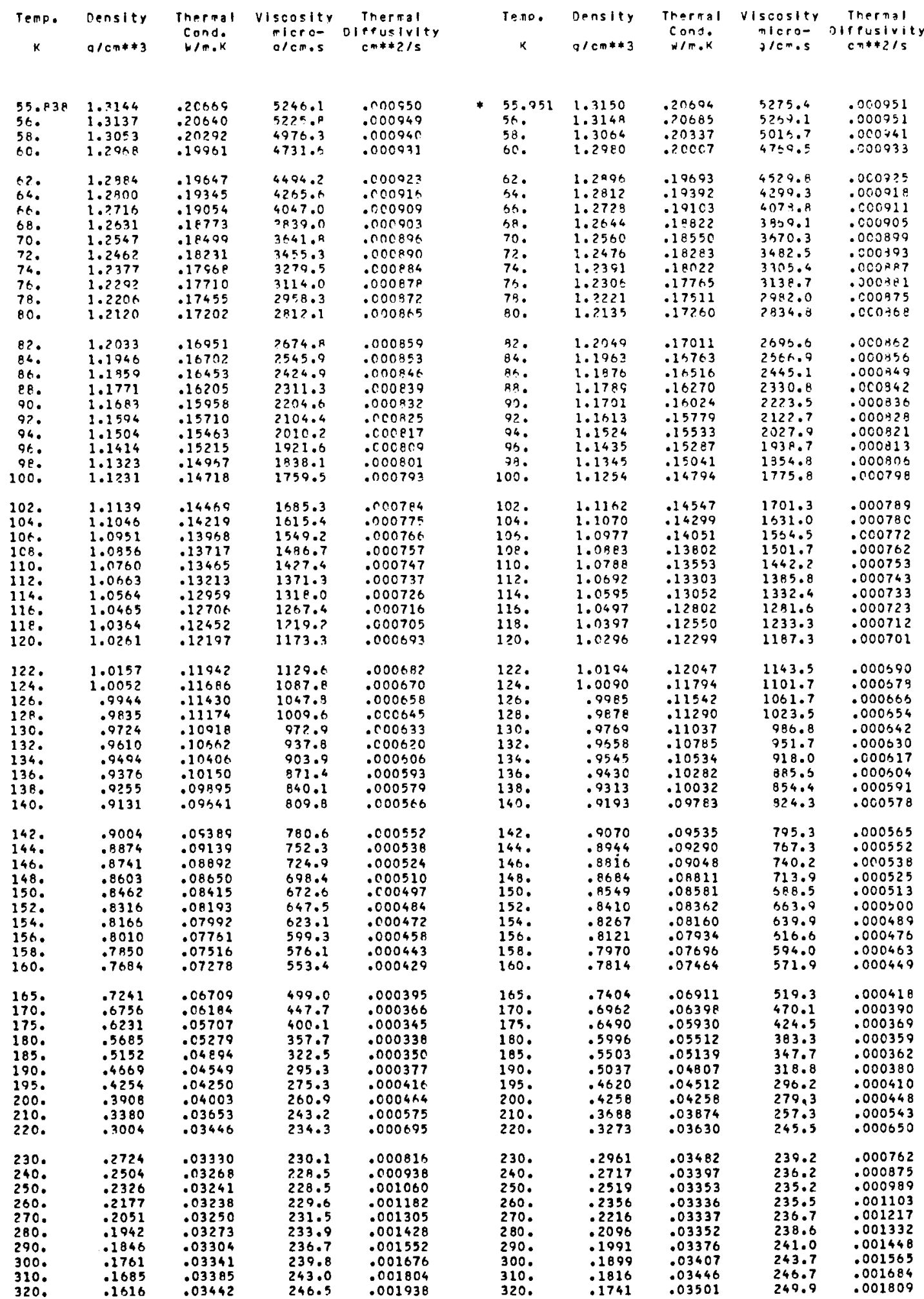

$14.0 \mathrm{MDa}$ ISObar 
Table 5. Transoort propertles of Oxven, Isobars, SI Units.

15.0 MP. Isobar

\begin{tabular}{|c|c|c|c|c|c|c|c|c|c|}
\hline $\begin{array}{c}\text { Teno. } \\
k\end{array}$ & $\begin{array}{l}\text { Density } \\
\text { g/cm**3 }\end{array}$ & $\begin{array}{l}\text { Thermal } \\
\text { Cond. } \\
\text { w/m.k }\end{array}$ & $\begin{array}{c}\text { VIscosity } \\
\text { mlere- } \\
\text { a/cmos }\end{array}$ & $\begin{array}{c}\text { Thermal } \\
\text { MffusIvIty } \\
\text { cm*\#/s }\end{array}$ & $\begin{array}{c}\text { Teno. } \\
k\end{array}$ & $\begin{array}{l}\text { Denslty } \\
0 / c \pi * 3\end{array}$ & $\begin{array}{l}\text { Thermol } \\
\text { Cond. } \\
\text { w/m.k }\end{array}$ & $\begin{array}{c}\text { VIscosity } \\
\text { mlero- } \\
0 / \mathrm{cm} \cdot \mathrm{s}\end{array}$ & $\begin{array}{c}\text { Thermal } \\
\text { oifeusivit } \\
\text { cm*2/s }\end{array}$ \\
\hline $\begin{array}{l}56.063 \\
58 . \\
6 C .\end{array}$ & $\begin{array}{l}1.3157 \\
1.3075 \\
1.2991\end{array}$ & $\begin{array}{l}.20718 \\
.20381 \\
.20052\end{array}$ & $\begin{array}{l}5304.5 \\
5057.3 \\
4907.5\end{array}$ & $\begin{array}{l}.000952 \\
.000943 \\
.000935\end{array}$ & $\begin{array}{l}50.176 \\
58 . \\
60 .\end{array}$ & $\begin{array}{l}1.3163 \\
1.3086 \\
1.3012\end{array}$ & $\begin{array}{l}.20742 \\
.70426 \\
.20098\end{array}$ & $\begin{array}{l}5333.4 \\
5098.0 \\
4945.7\end{array}$ & $\begin{array}{l}.000953 \\
.000944 \\
.000934\end{array}$ \\
\hline $\begin{array}{l}62 . \\
64 . \\
66 . \\
68 . \\
70 . \\
72 . \\
74 . \\
74 . \\
78 . \\
80 .\end{array}$ & $\begin{array}{l}1.2907 \\
1.2824 \\
1.2740 \\
1.2657 \\
1.2573 \\
1.2489 \\
1.2405 \\
1.2321 \\
1.2236 \\
1.2151\end{array}$ & $\begin{array}{l}119739 \\
.19460 \\
.19151 \\
118972 \\
.16600 \\
.18335 \\
.18075 \\
.17919 \\
.17567 \\
.17317\end{array}$ & $\begin{array}{l}4565.0 \\
4332.9 \\
4110.4 \\
3999 . ? \\
3699.0 \\
3529.7 \\
3331.3 \\
3163.5 \\
3005.7 \\
2857.5\end{array}$ & $\begin{array}{l}.000927 \\
.000920 \\
.000914 \\
.000907 \\
.000901 \\
.000 A 95 \\
.000849 \\
.000992 \\
.000 R 77 \\
.000871\end{array}$ & $\begin{array}{l}6 ? . \\
64: \\
64: \\
58 . \\
70 . \\
7 ? . \\
74: \\
76 . \\
78 . \\
90 .\end{array}$ & $\begin{array}{l}1.2919 \\
1.2834 \\
1.2752 \\
1.7369 \\
1.2585 \\
1.2502 \\
1.2419 \\
1.2235 \\
1.2251 \\
1.2166\end{array}$ & $\begin{array}{l}.19785 \\
.19486 \\
.19190 \\
.18921 \\
.19650 \\
.18357 \\
.18128 \\
.17874 \\
.17623 \\
.17375\end{array}$ & $\begin{array}{l}4301.4 \\
4366.7 \\
4142.5 \\
3929.4 \\
3727.5 \\
3536.9 \\
3357.3 \\
3138.2 \\
3329.3 \\
2890.1\end{array}$ & $\begin{array}{l}.000729 \\
.000722 \\
.00091 .6 \\
.000707 \\
.002793 \\
.000898 \\
.000347 \\
.000380 \\
.000330 \\
.000874\end{array}$ \\
\hline $\begin{array}{l}82 . \\
84 . \\
84 . \\
88 . \\
96 . \\
92 . \\
94 . \\
90 . \\
98 . \\
100 .\end{array}$ & $\begin{array}{l}1.2065 \\
1.1736 \\
1.1893 \\
1.1807 \\
1.1720 \\
1.1532 \\
1.1544 \\
1.1455 \\
1.1344 \\
1.1274\end{array}$ & $\begin{array}{l}.17070 \\
.16824 \\
116579 \\
115334 \\
.16090 \\
115846 \\
115403 \\
115359 \\
.15114 \\
.14 A 7 C\end{array}$ & $\begin{array}{l}2718.3 \\
2587.8 \\
2455.7 \\
2350.2 \\
2242.7 \\
2140.8 \\
3049.5 \\
1955.0 \\
1871.5 \\
1792.0\end{array}$ & 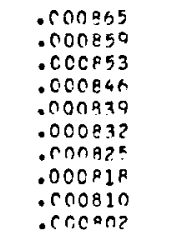 & $\begin{array}{l}92 . \\
84: \\
96: \\
89: \\
90 . \\
92: \\
94: \\
95: \\
99 . \\
100 .\end{array}$ & $\begin{array}{l}1.2081 \\
1.1996 \\
1.1910 \\
1.1474 \\
1.1738 \\
1.1251 \\
1.1534 \\
1.1476 \\
1.1387 \\
1.1798\end{array}$ & $\begin{array}{l}.17129 \\
.16884 \\
.16641 \\
.15396 \\
.15156 \\
.15914 \\
.15672 \\
.15429 \\
.15167 \\
.14044\end{array}$ & $\begin{array}{l}2740.1 \\
2638.7 \\
2485.4 \\
2359.5 \\
2251.0 \\
2155.0 \\
2053.1 \\
1972.7 \\
1898.1 \\
1898.2\end{array}$ & $\begin{array}{l}.000363 \\
.00006 ? \\
.000856 \\
.000849 \\
.000343 \\
.000935 \\
.000327 \\
.000322 \\
.000314 \\
.000207\end{array}$ \\
\hline $\begin{array}{l}102 . \\
104 . \\
104 . \\
108 \\
110 . \\
112 . \\
114 . \\
116 . \\
110 . \\
120 .\end{array}$ & $\begin{array}{l}1.2185 \\
1.1094 \\
1.1002 \\
1.0908 \\
1.0815 \\
1.0729 \\
1.0625 \\
1.0529 \\
1.0429 \\
1.0331\end{array}$ & $\begin{array}{l}.14424 \\
.14379 \\
.14133 \\
.13824 \\
.13639 \\
.13392 \\
.13144 \\
.12896 \\
.12547 \\
.12399\end{array}$ & $\begin{array}{l}1717.1 \\
1544.5 \\
1579.7 \\
1517.4 \\
1456.0 \\
1400.3 \\
1346.4 \\
1705.4 \\
1747.2 \\
1201.1\end{array}$ & $\begin{array}{l}.000796 \\
.000795 \\
.000777 \\
. r 00768 \\
.000759 \\
.000740 \\
.000739 \\
.000779 \\
.000719 \\
.00070\end{array}$ & $\begin{array}{l}102 . \\
104 . \\
104 . \\
1020 \\
110 . \\
112 . \\
114 . \\
115 . \\
119 . \\
120 .\end{array}$ & $\begin{array}{l}1.1209 \\
1.1118 \\
1.1027 \\
1.0035 \\
1.0842 \\
1.0748 \\
1.0554 \\
1.0558 \\
1.0462 \\
1.0364\end{array}$ & $\begin{array}{l}.14701 \\
.14458 \\
.14214 \\
.13969 \\
.13725 \\
.13479 \\
.13234 \\
.12988 \\
.12742 \\
.12496\end{array}$ & $\begin{array}{l}1732.9 \\
1351.9 \\
1574.9 \\
1531.4 \\
1471.4 \\
1414.5 \\
1360.7 \\
1379.5 \\
1201.0 \\
1214.7\end{array}$ & $\begin{array}{l}.006707 \\
.000790 \\
.00079 ? \\
.000773 \\
.000764 \\
.000755 \\
.000745 \\
.000735 \\
.000725 \\
.000715\end{array}$ \\
\hline $\begin{array}{l}122 . \\
124 . \\
126 . \\
128 . \\
130 . \\
132 . \\
134 . \\
136 . \\
138 . \\
140 .\end{array}$ & $\begin{array}{r}1.0230 \\
1.0129 \\
1.0725 \\
.9920 \\
.9913 \\
.7705 \\
.0594 \\
.9482 \\
.9308 \\
.9251\end{array}$ & $\begin{array}{l}.12149 \\
.119100 \\
.11451 \\
.11402 \\
.11154 \\
110905 \\
.10557 \\
.16416 \\
.10144 \\
.09970\end{array}$ & $\begin{array}{r}1157.2 \\
1115.3 \\
1375.3 \\
1037.1 \\
1000.5 \\
965.4 \\
931.7 \\
890.4 \\
968.3 \\
839.4\end{array}$ & $\begin{array}{l}.000497 \\
.000586 \\
.000675 \\
.000643 \\
.000451 \\
.000639 \\
.000527 \\
.000615 \\
.000602 \\
.000590\end{array}$ & $\begin{array}{l}127 . \\
1240^{\circ} \\
1260^{\circ} \\
1280^{\circ} \\
1300^{\circ} \\
1320^{\circ} \\
1340^{\circ} \\
1330^{\circ} \\
138 . \\
140 .\end{array}$ & $\begin{array}{l}1.0265 \\
1.0165 \\
1.0063 \\
.9960 \\
.9856 \\
.9749 \\
.9642 \\
.9532 \\
.9421 \\
.9307\end{array}$ & $\begin{array}{l}.12250 \\
.12004 \\
.11758 \\
.11512 \\
.11267 \\
.11022 \\
.10778 \\
.10535 \\
.10293 \\
.10052\end{array}$ & $\begin{array}{r}1170.7 \\
1128.7 \\
1088.7 \\
1050.4 \\
1013.8 \\
978.7 \\
945.1 \\
912.9 \\
981.9 \\
852.0\end{array}$ & $\begin{array}{l}.000705 \\
.000594 \\
.000483 \\
.002572 \\
.000400 \\
.000649 \\
.000537 \\
.000525 \\
.000513 \\
.000501\end{array}$ \\
\hline $\begin{array}{l}142 . \\
144 . \\
148 . \\
149 . \\
150 . \\
152 . \\
154 . \\
156 . \\
158 . \\
160 .\end{array}$ & $\begin{array}{l}.9137 \\
.9211 \\
.8887 \\
.8760 \\
.8930 \\
.9497 \\
.8361 \\
.8221 \\
.8078 \\
.7931\end{array}$ & $\begin{array}{l}.04577 \\
.09436 \\
.09198 \\
.08945 \\
.08739 \\
.08522 \\
.08322 \\
.08099 \\
.07948 \\
.07442\end{array}$ & $\begin{array}{l}900.5 \\
781.7 \\
754.8 \\
728.9 \\
703.7 \\
579.4 \\
255.8 \\
332.9 \\
610.7 \\
580.2\end{array}$ & $\begin{array}{l}.000577 \\
.000545 \\
.000552 \\
.000540 \\
.000527 \\
.00051 t \\
.000565 \\
.000497 \\
.000490 \\
.000448\end{array}$ & $\begin{array}{l}142 . \\
144 . \\
145 . \\
148 . \\
150 . \\
152 . \\
154 . \\
159 . \\
159 . \\
150 .\end{array}$ & $\begin{array}{l}.9191 \\
.9074 \\
.8953 \\
.8831 \\
.8704 \\
.8578 \\
.8447 \\
.8314 \\
.8178 \\
.8038\end{array}$ & $\begin{array}{l}.09813 \\
.09576 \\
.09343 \\
.09114 \\
.08891 \\
.08577 \\
.08477 \\
.08258 \\
.08032 \\
.07811\end{array}$ & $\begin{array}{l}823.3 \\
775.7 \\
759.0 \\
743.2 \\
718.3 \\
694.3 \\
671.0 \\
548.4 \\
526.5 \\
605.3\end{array}$ & $\begin{array}{l}.000549 \\
.000577 \\
.000565 \\
.000553 \\
.000541 \\
.000530 \\
.000520 \\
.000508 \\
.000495 \\
.000485\end{array}$ \\
\hline $\begin{array}{l}165 . \\
170 . \\
1750 \\
180 \\
185 \\
190 \\
195 \\
200 \\
210 \\
220 .\end{array}$ & $\begin{array}{l}.7548 \\
.7130 \\
.6705 \\
.3254 \\
.5799 \\
.5357 \\
.6968 \\
.4589 \\
.3993 \\
.3536\end{array}$ & $\begin{array}{l}.07101 \\
.06598 \\
.06179 \\
.05726 \\
.05350 \\
.05034 \\
.04745 \\
.04491 \\
.04028 \\
.03814\end{array}$ & $\begin{array}{l}537.9 \\
490.2 \\
446.1 \\
406.1 \\
370.8 \\
340.9 \\
316.7 \\
? 97.7 \\
272.0 \\
257.2\end{array}$ & $\begin{array}{l}.000438 \\
.000413 \\
.000392 \\
.000390 \\
.000378 \\
.000388 \\
.000410 \\
.000441 \\
.00059 ? \\
.000617\end{array}$ & $\begin{array}{l}155 . \\
170 . \\
175 . \\
190 . \\
185 . \\
190 . \\
1950 \\
200 . \\
210 . \\
270 .\end{array}$ & $\begin{array}{l}.7676 \\
.7293 \\
.5991 \\
.6474 \\
.0050 \\
.5634 \\
.5239 \\
.4776 \\
.4264 \\
.3791\end{array}$ & $\begin{array}{l}.07282 \\
.03799 \\
.06335 \\
.05927 \\
.05563 \\
.05741 \\
.04955 \\
.04703 \\
.04291 \\
.03945\end{array}$ & $\begin{array}{l}555.1 \\
538.6 \\
455.7 \\
426.6 \\
391.8 \\
351.8 \\
335.3 \\
315.8 \\
285.8 \\
250.2\end{array}$ & $\begin{array}{l}.000457 \\
.000433 \\
.000413 \\
.000394 \\
.000394 \\
.000400 \\
.000415 \\
.000440 \\
.000508 \\
.000592\end{array}$ \\
\hline $\begin{array}{l}230 . \\
240 . \\
250 \\
260 \\
270 \\
280 \\
290 \\
300 \\
310 . \\
320 .\end{array}$ & $\begin{array}{l}.7195 \\
.2028 \\
.2717 \\
.2533 \\
.7391 \\
.2251 \\
.2136 \\
.2036 \\
.1946 \\
.1865\end{array}$ & $\begin{array}{l}.03639 \\
.03531 \\
.03438 \\
.03437 \\
.03427 \\
.03433 \\
.03449 \\
.03474 \\
.03509 \\
.03530\end{array}$ & $\begin{array}{l}249.9 \\
244.3 \\
? 42.7 \\
241.7 \\
24 ? . ? \\
243.8 \\
245.5 \\
247.0 \\
250.5 \\
263.5\end{array}$ & $\begin{array}{l}.000719 \\
.000824 \\
.000930 \\
.001037 \\
.001144 \\
.001252 \\
.001340 \\
.001464 \\
.001581 \\
.001700\end{array}$ & $\begin{array}{l}230 . \\
240 . \\
250 . \\
250 . \\
270 . \\
230 . \\
290 . \\
300 . \\
310 . \\
320 .\end{array}$ & $\begin{array}{l}.3425 \\
.3136 \\
.2903 \\
.2709 \\
.2545 \\
.2406 \\
.2280 \\
.2172 \\
.2075 \\
.1989\end{array}$ & $\begin{array}{l}.03795 \\
.03666 \\
.03586 \\
.03541 \\
.03520 \\
.03516 \\
.03524 \\
.03542 \\
.03572 \\
.03620\end{array}$ & $\begin{array}{l}258.5 \\
252.7 \\
249.5 \\
248.1 \\
747.9 \\
248.7 \\
252.2 \\
252.1 \\
254.5 \\
257.1\end{array}$ & $\begin{array}{l}.000585 \\
.000783 \\
.000392 \\
.000781 \\
.001032 \\
.001183 \\
.001285 \\
.001388 \\
.001493 \\
.001405\end{array}$ \\
\hline
\end{tabular}

$16.0 \mathrm{MPa}$ Is obar 
Table 5. Transoort Pronertles of Qxygen, Isobars, SI Units.

77. MPa ISODER

\begin{tabular}{|c|c|c|c|c|c|c|c|c|c|}
\hline Tens. & $\begin{array}{l}\text { Density } \\
\operatorname{cosen} * 3\end{array}$ & $\begin{array}{l}\text { Therral } \\
\text { Cond. } \\
\text { w/mek }\end{array}$ & $\begin{array}{c}\text { Viscosity } \\
\text { mierc- } \\
\text { o/cmos }\end{array}$ & $\begin{array}{c}\text { Thermal } \\
\text { olffusivity } \\
\text { cm**2/s }\end{array}$ & $\begin{array}{c}\text { Tend. } \\
k\end{array}$ & $\begin{array}{l}\text { Density } \\
\text { g/cm } * 3\end{array}$ & $\begin{array}{l}\text { Thermal } \\
\text { Cond. } \\
\text { w/m.k }\end{array}$ & $\begin{array}{c}\text { VIscosity } \\
\text { mIcro- } \\
\text { g/cmes }\end{array}$ & $\begin{array}{c}\text { Thermsi } \\
\text { Oiffusivit } \\
\text { cm } * \neq 2 / \mathrm{s}\end{array}$ \\
\hline $\begin{array}{l}53.2 \times 8 \\
5 \% . \\
6 n .\end{array}$ & $\begin{array}{l}1.3167 \\
1.3097 \\
1.3012\end{array}$ & $\begin{array}{l}.20746 \\
.20470 \\
.20143\end{array}$ & $\begin{array}{l}5362 . ? \\
3139.9 \\
4283.9\end{array}$ & $\begin{array}{l}.000954 \\
.000946 \\
.000938\end{array}$ & $\begin{array}{l}56.399 \\
58 . \\
60 .\end{array}$ & $\begin{array}{l}1.3175 \\
1.3108 \\
1.3025\end{array}$ & $\begin{array}{l}.20790 \\
.20515 \\
.20187\end{array}$ & $\begin{array}{l}5370.9 \\
5179.8 \\
4922.2\end{array}$ & $\begin{array}{l}.000955 \\
.000948 \\
.000940\end{array}$ \\
\hline $\begin{array}{l}42 . \\
+4 . \\
64 . \\
39 . \\
70 . \\
72 . \\
76 . \\
75 . \\
79 . \\
80 .\end{array}$ & $\begin{array}{l}1.2930 \\
1.2847 \\
1.2745 \\
1.2582 \\
1.2597 \\
1.2514 \\
1.2432 \\
1.2349 \\
1.7255 \\
1.2181\end{array}$ & $\begin{array}{r}.19831 \\
.19533 \\
.19747 \\
.18970 \\
.18700 \\
.18439 \\
.18191 \\
.17928 \\
.17578 \\
.17432\end{array}$ & $\begin{array}{l}4637.3 \\
4400.5 \\
4174.4 \\
3959.5 \\
3756.2 \\
3564 . ? \\
3383.2 \\
2922.0 \\
3053.0 \\
2902.8\end{array}$ & $\begin{array}{l}.000931 \\
.000924 \\
.000918 \\
.000912 \\
.000906 \\
.000900 \\
.000994 \\
.000889 \\
.000883 \\
.000877\end{array}$ & $\begin{array}{l}62 . \\
64 . \\
66 . \\
68 . \\
70 . \\
72 . \\
74 . \\
76 . \\
78 . \\
80 .\end{array}$ & $\begin{array}{l}1.2942 \\
1.2859 \\
1.2777 \\
1.2694 \\
1.2611 \\
1.2529 \\
1.2446 \\
1.2363 \\
1.2280 \\
1.2196\end{array}$ & $\begin{array}{l}.19877 \\
.19580 \\
.19294 \\
.19018 \\
.18750 \\
.18489 \\
.18233 \\
.17981 \\
.17733 \\
.17488\end{array}$ & $\begin{array}{l}4673.2 \\
4434.3 \\
4206.4 \\
3999.8 \\
3784.9 \\
3591.4 \\
3409.1 \\
3237.7 \\
3076.6 \\
2925.4\end{array}$ & $\begin{array}{l}.000932 \\
.000926 \\
.000920 \\
.000914 \\
.000908 \\
.000902 \\
.000997 \\
.000891 \\
.000886 \\
.000860\end{array}$ \\
\hline $\begin{array}{l}8 ? . \\
84 . \\
86 . \\
98 . \\
98 . \\
9 ? . \\
94 . \\
96 . \\
98 . \\
1 C C .\end{array}$ & $\begin{array}{l}1.2007 \\
1.201 ? \\
1.1077 \\
1.1842 \\
1.1756 \\
1.1573 \\
1.1583 \\
1.1406 \\
1.1408 \\
1.1320\end{array}$ & $\begin{array}{l}.17187 \\
.16944 \\
.16702 \\
.16461 \\
.16220 \\
.15990 \\
.15740 \\
.15500 \\
.15259 \\
.15018\end{array}$ & $\begin{array}{l}2761.8 \\
2629.5 \\
2505.4 \\
2380.0 \\
2279.7 \\
2177.0 \\
2020.6 \\
1989.9 \\
1904.6 \\
1924.3\end{array}$ & $\begin{array}{l}.000871 \\
.000865 \\
.000859 \\
.000853 \\
.000846 \\
.000940 \\
.000833 \\
.000829 \\
.000819 \\
.000811\end{array}$ & $\begin{array}{l}82 . \\
84 . \\
86 . \\
88 . \\
90 . \\
92 . \\
94 . \\
96 . \\
98 . \\
100 .\end{array}$ & $\begin{array}{l}1.2112 \\
1.2028 \\
1.1944 \\
1.1859 \\
1.1774 \\
1.1688 \\
1.1602 \\
1.1515 \\
1.1428 \\
1.1341\end{array}$ & $\begin{array}{l}.17245 \\
.17004 \\
.16763 \\
.16524 \\
.16285 \\
.16046 \\
.15808 \\
.15569 \\
.15330 \\
.15091\end{array}$ & $\begin{array}{l}2783.5 \\
2650.4 \\
2525.5 \\
2408.3 \\
2298.3 \\
2195.1 \\
2098.1 \\
2000.9 \\
1921.1 \\
1840.3\end{array}$ & $\begin{array}{l}.000874 \\
.000868 \\
.000862 \\
.000856 \\
.000850 \\
.000843 \\
.000837 \\
.000830 \\
.000823 \\
.000815\end{array}$ \\
\hline $\begin{array}{l}102 . \\
104 . \\
104 . \\
108 . \\
110 . \\
1120 \\
114 . \\
116 . \\
118 . \\
120 .\end{array}$ & $\begin{array}{l}1.1231 \\
1.1141 \\
1.1051 \\
1.0960 \\
1.0548 \\
1.0773 \\
1.0382 \\
1.0588 \\
1.0603 \\
1.0396\end{array}$ & $\begin{array}{l}.14777 \\
.14535 \\
.14204 \\
.14051 \\
.13809 \\
.13566 \\
.13323 \\
.13070 \\
.12836 \\
.12593\end{array}$ & $\begin{array}{l}1748.6 \\
1677.2 \\
1609.8 \\
1546.1 \\
1485.8 \\
1478.7 \\
1374.6 \\
1323.3 \\
1274.6 \\
1228.2\end{array}$ & $\begin{array}{l}.000803 \\
.000705 \\
.000787 \\
.000778 \\
.000770 \\
.000761 \\
.000751 \\
.000742 \\
.000732 \\
.000772\end{array}$ & $\begin{array}{l}102 . \\
104 . \\
106 . \\
108 . \\
110 . \\
112 . \\
114 . \\
116 . \\
118 . \\
120 .\end{array}$ & $\begin{array}{l}1.1253 \\
1.1164 \\
1.1075 \\
1.0985 \\
1.0894 \\
1.0802 \\
1.0710 \\
1.0617 \\
1.0523 \\
1.0428\end{array}$ & $\begin{array}{l}.14852 \\
.14613 \\
.14373 \\
.14132 \\
.13892 \\
.13651 \\
.13410 \\
.13169 \\
.12928 \\
.12687\end{array}$ & $\begin{array}{l}1764.2 \\
1692.4 \\
1624.6 \\
1560.6 \\
1500.1 \\
1442.8 \\
1388.5 \\
1336.9 \\
1288.0 \\
1241.5\end{array}$ & $\begin{array}{l}.000808 \\
.000300 \\
.000792 \\
.000783 \\
.000775 \\
.000766 \\
.000757 \\
.000748 \\
.000738 \\
.000729\end{array}$ \\
\hline $\begin{array}{l}1220^{\circ} \\
124 . \\
126 . \\
128 . \\
1300^{\circ} \\
1320^{\circ} \\
134 . \\
136 . \\
130^{\circ} \\
140 .\end{array}$ & $\begin{array}{l}1.0290 \\
1.0200 \\
1.0100 \\
.9999 \\
.9897 \\
.9793 \\
.9587 \\
.9580 \\
.9471 \\
.9365\end{array}$ & $\begin{array}{l}.12349 \\
.12106 \\
.11863 \\
.11120 \\
.11378 \\
.11136 \\
.10946 \\
.10656 \\
.10417 \\
.10180\end{array}$ & $\begin{array}{r}1184.1 \\
1142.0 \\
1101.9 \\
1053.0 \\
1026.9 \\
991.8 \\
958.2 \\
926.0 \\
895.1 \\
865.3\end{array}$ & $\begin{array}{l}.000712 \\
.000701 \\
.000691 \\
.000680 \\
.000669 \\
.000658 \\
.000646 \\
.000635 \\
.000623 \\
.000612\end{array}$ & $\begin{array}{l}1220^{\circ} \\
124^{\circ} \\
126^{\circ} \\
128^{\circ} \\
1300^{\circ} \\
1320^{\circ} \\
1340^{\circ} \\
1360^{\circ} \\
130^{\circ} \\
140^{\circ}\end{array}$ & $\begin{array}{r}1.0332 \\
1.0235 \\
1.0137 \\
1.0037 \\
.9937 \\
.9834 \\
.9731 \\
.9626 \\
.9520 \\
.9422\end{array}$ & $\begin{array}{l}.12446 \\
.12206 \\
.11966 \\
.11726 \\
.11486 \\
.11248 \\
.111010 \\
.10774 \\
.10538 \\
.10305\end{array}$ & $\begin{array}{r}1197.2 \\
1155.1 \\
1114.9 \\
1076.5 \\
1039.8 \\
1004.7 \\
971.1 \\
938.9 \\
908.0 \\
878.3\end{array}$ & $\begin{array}{l}.000719 \\
.000709 \\
.000698 \\
.000688 \\
.000677 \\
.000666 \\
.000655 \\
.000644 \\
.000633 \\
.000622\end{array}$ \\
\hline $\begin{array}{l}142 . \\
1440 \\
1460 \\
148 . \\
150 . \\
1520^{\circ} \\
154 . \\
156 . \\
158 . \\
160^{\circ} .\end{array}$ & $\begin{array}{l}.924 R \\
.9133 \\
.9017 \\
.8273 \\
.8777 \\
.8654 \\
.8528 \\
.8400 \\
.8270 \\
.8136\end{array}$ & $\begin{array}{l}.09945 \\
.09712 \\
.09482 \\
.09257 \\
.00037 \\
.08825 \\
.08626 \\
.08411 \\
.08189 \\
.07973\end{array}$ & $\begin{array}{l}836.7 \\
809.2 \\
782.7 \\
757.1 \\
732.4 \\
708.5 \\
385.4 \\
663.1 \\
341.5 \\
620.6\end{array}$ & $\begin{array}{l}.000600 \\
.000588 \\
.000577 \\
.000566 \\
.000554 \\
.000544 \\
.000534 \\
.000523 \\
.000511 \\
.000500\end{array}$ & $\begin{array}{l}142 . \\
144 . \\
145 . \\
148 . \\
150 . \\
152 . \\
154 . \\
150^{\circ} \\
158 . \\
160 .\end{array}$ & $\begin{array}{l}.9302 \\
.9190 \\
.9077 \\
.8962 \\
.8845 \\
.8725 \\
.8604 \\
.8481 \\
.8355 \\
.8227\end{array}$ & $\begin{array}{l}.10073 \\
.09844 \\
.09617 \\
.09395 \\
.09178 \\
.08969 \\
.08771 \\
.08559 \\
.08341 \\
.08129\end{array}$ & $\begin{array}{l}849.8 \\
822.3 \\
795.9 \\
770.5 \\
745.9 \\
722.2 \\
699.3 \\
677.2 \\
655.8 \\
635.2\end{array}$ & $\begin{array}{l}.000611 \\
.000500 \\
.000588 \\
.000577 \\
.000567 \\
.000556 \\
.000547 \\
.000536 \\
.000525 \\
.000515\end{array}$ \\
\hline $\begin{array}{l}165 . \\
170 . \\
1750 \\
180 . \\
185 . \\
190 . \\
1950 \\
200 . \\
210 . \\
220 .\end{array}$ & $\begin{array}{l}.7792 \\
.7431 \\
.7054 \\
.6564 \\
.6269 \\
.5876 \\
.5497 \\
.5142 \\
.4576 \\
.4035\end{array}$ & $\begin{array}{l}.07454 \\
.06959 \\
.05522 \\
.05117 \\
.05754 \\
.05433 \\
.05149 \\
.04898 \\
.04482 \\
.04171\end{array}$ & $\begin{array}{l}571.2 \\
525.6 \\
483.7 \\
445.4 \\
411.0 \\
380.8 \\
354.9 \\
333.4 \\
301.7 \\
281.6\end{array}$ & $\begin{array}{l}.000474 \\
.000451 \\
.000431 \\
.000418 \\
.000411 \\
.000413 \\
.000423 \\
.000442 \\
.000500 \\
.000574\end{array}$ & $\begin{array}{l}165 . \\
170 . \\
175 . \\
180 . \\
185 . \\
190 . \\
195 . \\
200 . \\
210 . \\
220 .\end{array}$ & $\begin{array}{l}.7898 \\
.7555 \\
.7199 \\
.6833 \\
.6460 \\
.6089 \\
.5727 \\
.5382 \\
.4770 \\
.4268\end{array}$ & $\begin{array}{l}.07619 \\
.07142 \\
.06700 \\
.06298 \\
.05936 \\
.05615 \\
.05331 \\
.05079 \\
.04660 \\
.04340\end{array}$ & $\begin{array}{l}508.4 \\
541.6 \\
500.4 \\
462.8 \\
428.8 \\
398.7 \\
372.5 \\
350.2 \\
316.3 \\
294.0\end{array}$ & $\begin{array}{l}.000490 \\
.000468 \\
.000449 \\
.000435 \\
.000427 \\
.000426 \\
.000433 \\
.000448 \\
.000496 \\
.000562\end{array}$ \\
\hline $\begin{array}{l}230 . \\
240 . \\
250 . \\
260 \\
270 \\
280 \\
200 \\
300 \\
310 . \\
320 .\end{array}$ & $\begin{array}{l}.3649 \\
.3341 \\
.3090 \\
.2882 \\
.2706 \\
.2555 \\
.2423 \\
.2307 \\
.2203 \\
.2110\end{array}$ & $\begin{array}{l}.03951 \\
.03803 \\
.03706 \\
.0364 t \\
.03514 \\
.03600 \\
.03601 \\
.03612 \\
.03636 \\
.03381\end{array}$ & $\begin{array}{l}269.1 \\
261.5 \\
257.0 \\
254.7 \\
253.8 \\
254.0 \\
255.0 \\
256.6 \\
258.6 \\
260.9\end{array}$ & $\begin{array}{l}.000659 \\
.000749 \\
.000841 \\
.000935 \\
.001029 \\
.0011125 \\
.001221 \\
.001317 \\
.001417 \\
.001523\end{array}$ & $\begin{array}{l}230 . \\
240 . \\
250 . \\
260 . \\
270^{\circ} \\
280 . \\
290^{\circ} \\
300^{\circ} \\
310^{\circ} \\
320^{\circ}\end{array}$ & $\begin{array}{r}.3865 \\
.3540 \\
.3275 \\
.3054 \\
.2866 \\
.2705 \\
.2564 \\
.2441 \\
.2330 \\
.2232\end{array}$ & $\begin{array}{l}.04104 \\
.03939 \\
.03826 \\
.03753 \\
.03709 \\
.03686 \\
.03678 \\
.03683 \\
.03701 \\
.03743\end{array}$ & $\begin{array}{l}279.6 \\
270.4 \\
264.8 \\
261.8 \\
259.9 \\
259.5 \\
260.0 \\
261.1 \\
262.8 \\
264.8\end{array}$ & $\begin{array}{l}.000638 \\
.000721 \\
.000807 \\
.000895 \\
.000984 \\
.001074 \\
.001165 \\
.001256 \\
.001350 \\
.001451\end{array}$ \\
\hline
\end{tabular}

18.0 MPa Isobar 
Table 5. Transport Properties of Exygen, Isobars, SI Unlts.

19.0 MOg Isobar

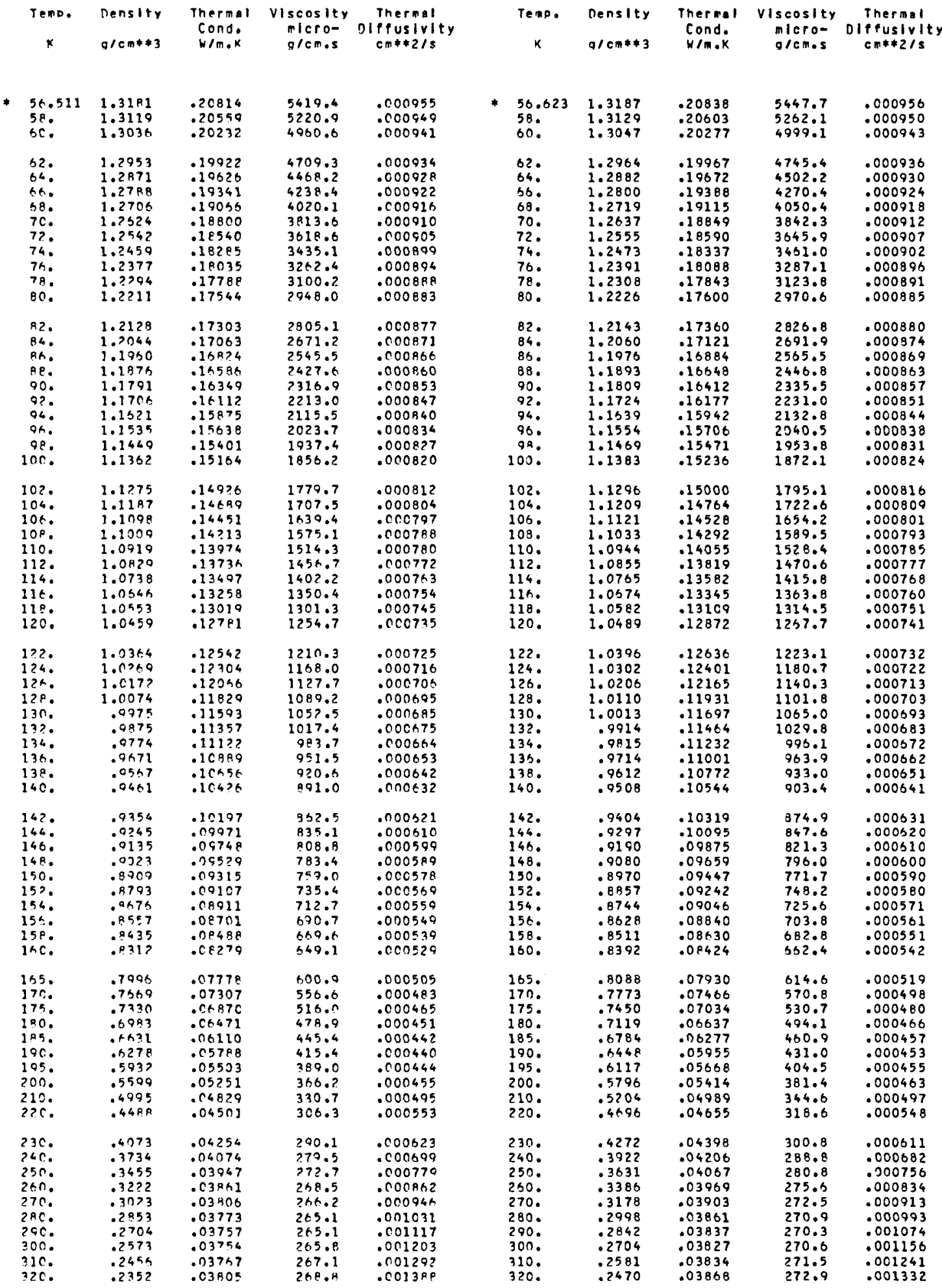

$20.0 \mathrm{MPg}$ Isober 
Table 5. Transoort propertles of Exygen, Isobars, SI Units.

21.0 MPA Isonar

22.0 YPa Isobar

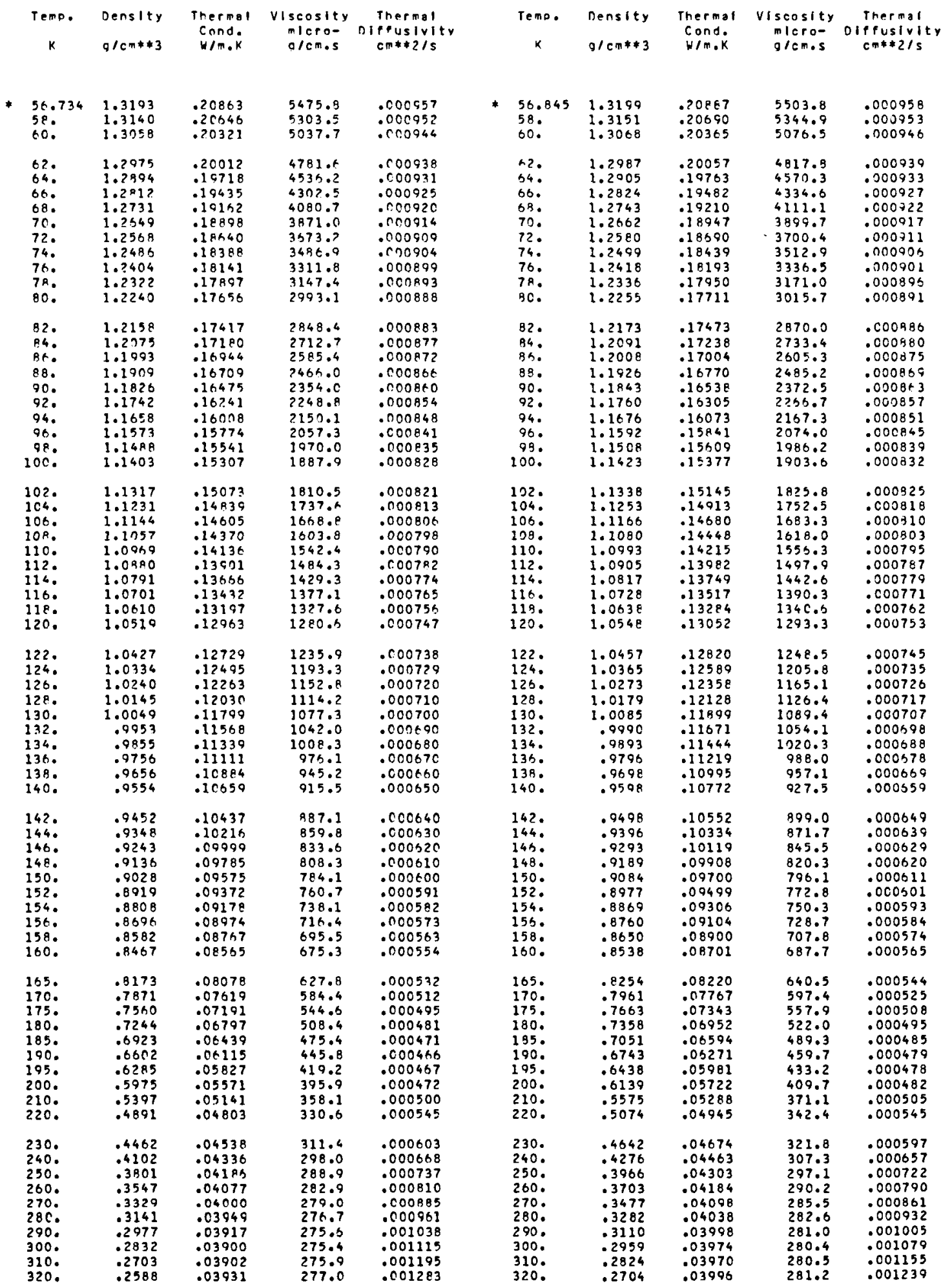

* Two Phase Boundary 
Tahle 5. Transoort Dropertles of Cxyaen, Isobars, SI Unlts.

23.0 MPE IsObar

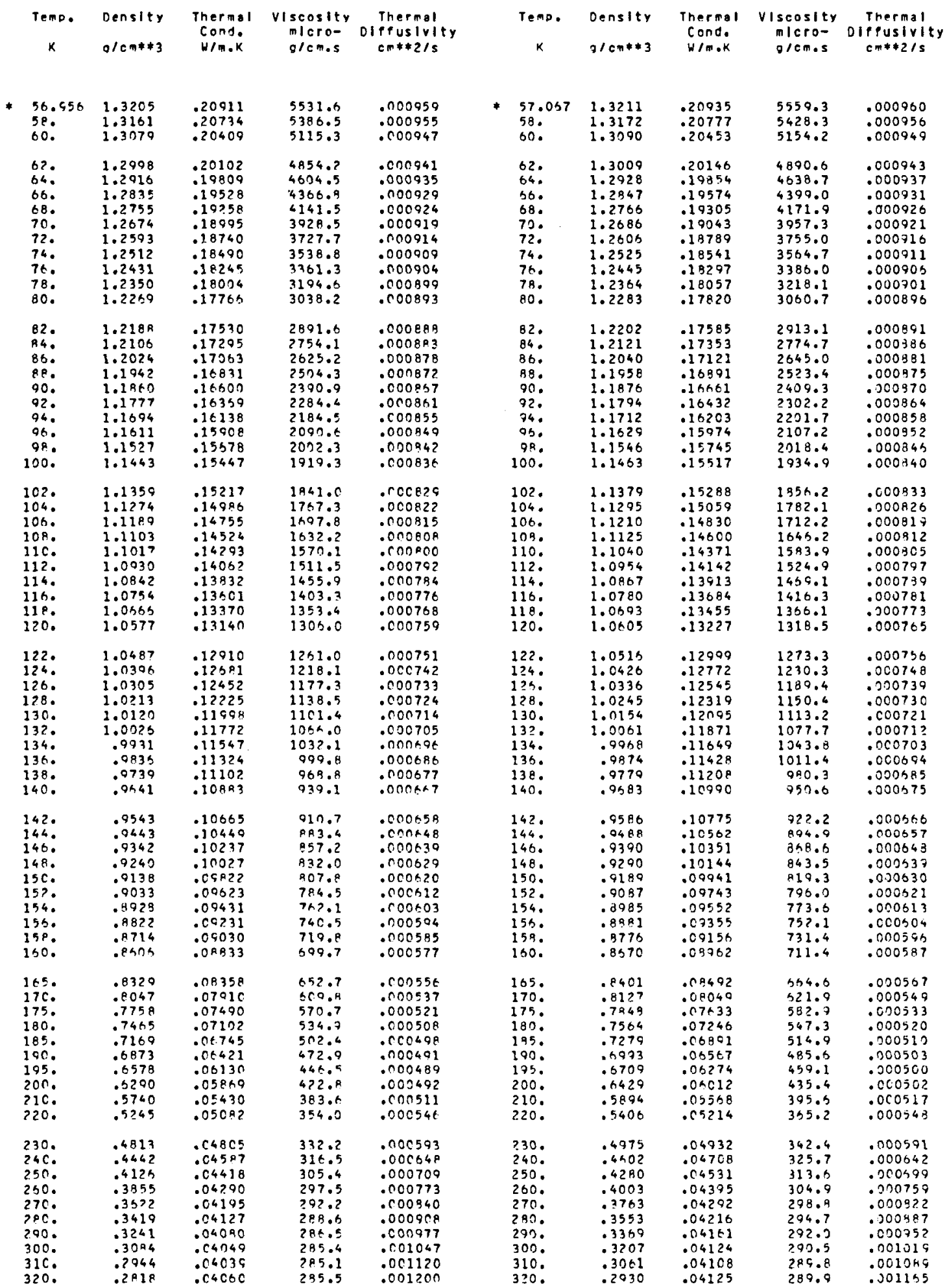

$24.0 \mathrm{MPa} \mathrm{Is00ar}$

* Two Phase Boundary 
Table 5. Transdort Propertles of oxygen, Isobars, si Units.

25.0 MPa Is Obar

\begin{tabular}{|c|c|c|c|c|c|c|c|c|c|}
\hline $\begin{array}{c}\text { Teno. } \\
k\end{array}$ & $\begin{array}{l}\text { Density } \\
\text { alcm**3 }\end{array}$ & $\begin{array}{l}\text { Therral } \\
\text { conds } \\
\text { W/mok }\end{array}$ & $\begin{array}{c}\text { Viscosity } \\
\text { micro- } \\
2 / c \pi .5\end{array}$ & $\begin{array}{c}\text { Thermal } \\
\text { Diffusivity } \\
\text { cm } * 2 / s\end{array}$ & $\begin{array}{c}\text { Temo. } \\
k\end{array}$ & $\begin{array}{l}\text { Density } \\
g / \mathrm{Cm} * 3\end{array}$ & $\begin{array}{l}\text { Thermal } \\
\text { Cond. } \\
\text { W/mok }\end{array}$ & $\begin{array}{c}\text { Viscosity } \\
\text { mlero- } \\
\text { g/cmos }\end{array}$ & $\begin{array}{c}\text { Thermal } \\
\text { DIffusivity } \\
\text { cm*21s }\end{array}$ \\
\hline $\begin{array}{l}57.178 \\
5 \% . \\
4 C .\end{array}$ & $\begin{array}{l}1.3213 \\
1.3183 \\
1.3101\end{array}$ & $\begin{array}{l}.20950 \\
.20820 \\
.20407\end{array}$ & $\begin{array}{l}5586.8 \\
5470.1 \\
5193.2\end{array}$ & $\begin{array}{l}.000960 \\
.000957 \\
.000950\end{array}$ & $\begin{array}{l}57.288 \\
59 . \\
60 .\end{array}$ & $\begin{array}{l}1.3222 \\
1.3193 \\
1.3111\end{array}$ & $\begin{array}{l}.20983 \\
.20863 \\
.20540\end{array}$ & $\begin{array}{l}5614.1 \\
5512.1 \\
5232.3\end{array}$ & $\begin{array}{l}.000961 \\
.000959 \\
.000952\end{array}$ \\
\hline $\begin{array}{l}+9 . \\
64 . \\
66 . \\
6 \% . \\
70 . \\
72 . \\
74 . \\
76 . \\
78 . \\
80 .\end{array}$ & $\begin{array}{l}1.3020 \\
1.2939 \\
1.2938 \\
1.2778 \\
1.2508 \\
1.2618 \\
1.2539 \\
1.2458 \\
1.2378 \\
1.2707\end{array}$ & $\begin{array}{l}.70101 \\
.19899 \\
.19670 \\
.19352 \\
.19091 \\
.19838 \\
.19591 \\
.18349 \\
.18110 \\
.17874\end{array}$ & $\begin{array}{l}4977.1 \\
4572.9 \\
4431.3 \\
4702.3 \\
3986.1 \\
2782.4 \\
3570.7 \\
3410.7 \\
3241.7 \\
3083.2\end{array}$ & $\begin{array}{l}.000944 \\
.000938 \\
.000933 \\
.000928 \\
.000923 \\
.000918 \\
.000913 \\
.000908 \\
.000904 \\
.000890\end{array}$ & $\begin{array}{l}62 . \\
64 . \\
68 . \\
68 . \\
70 . \\
72 . \\
74 . \\
76 . \\
78 . \\
80 .\end{array}$ & $\begin{array}{l}1.3030 \\
1.3950 \\
1.2870 \\
1.2790 \\
1.2710 \\
1.2631 \\
1.2551 \\
1.2471 \\
1.2391 \\
1.2311\end{array}$ & $\begin{array}{l}.20235 \\
.19944 \\
.19666 \\
.19398 \\
.19139 \\
.18887 \\
.18641 \\
.18400 \\
.18162 \\
.17928\end{array}$ & $\begin{array}{l}4963.7 \\
4707.3 \\
4463.6 \\
4232.8 \\
4014.9 \\
3809.7 \\
3616.7 \\
3435.4 \\
3265.2 \\
3105.7\end{array}$ & $\begin{array}{l}.000946 \\
.000940 \\
.000935 \\
.000930 \\
.000925 \\
.000920 \\
.000915 \\
.000911 \\
.000906 \\
.000901\end{array}$ \\
\hline $\begin{array}{l}82 . \\
94 . \\
84 . \\
88 . \\
90 . \\
92 . \\
94 . \\
96 . \\
98 . \\
100 .\end{array}$ & $\begin{array}{l}1.2217 \\
1.2136 \\
1.2055 \\
1.1974 \\
1.1993 \\
1.1811 \\
1.1730 \\
1.1647 \\
1.1565 \\
1.1472\end{array}$ & $\begin{array}{l}.17841 \\
.17409 \\
.17180 \\
.16951 \\
.14722 \\
.16495 \\
.16257 \\
.15040 \\
115813 \\
.15595\end{array}$ & $\begin{array}{l}2934.7 \\
2795.4 \\
2564.8 \\
2542.4 \\
2427.5 \\
2310.9 \\
2218.7 \\
2123.7 \\
2034.4 \\
1950.4\end{array}$ & $\begin{array}{l}.000894 \\
.000889 \\
.000884 \\
.000878 \\
.000873 \\
.000867 \\
.000862 \\
.000856 \\
.000850 \\
.000844\end{array}$ & $\begin{array}{l}82 . \\
84 . \\
80 . \\
88 . \\
90 . \\
92 . \\
94 . \\
96 . \\
98 . \\
100 .\end{array}$ & $\begin{array}{l}1.2231 \\
1.2151 \\
1.2071 \\
1.1990 \\
1.1909 \\
1.1828 \\
1.1747 \\
1.1665 \\
1.1584 \\
1.1501\end{array}$ & $\begin{array}{l}.17696 \\
.17466 \\
.17237 \\
.17010 \\
.16783 \\
.16557 \\
.16331 \\
.16105 \\
.15879 \\
.15653\end{array}$ & $\begin{array}{l}2956.2 \\
2816.0 \\
2684.6 \\
2531.4 \\
2445.9 \\
2337.5 \\
2235.8 \\
2140.2 \\
2050.4 \\
1965.9\end{array}$ & $\begin{array}{l}.000896 \\
.000892 \\
.000887 \\
.000881 \\
.000875 \\
.000871 \\
.000865 \\
.000859 \\
.000853 \\
.000847\end{array}$ \\
\hline $\begin{array}{l}102 . \\
104 . \\
104 . \\
105 . \\
110 . \\
1120 \\
114 . \\
116 . \\
119 . \\
120 .\end{array}$ & $\begin{array}{l}1.1309 \\
1.1316 \\
1.1232 \\
1.1149 \\
1.1043 \\
1.0778 \\
1.0992 \\
1.0406 \\
1.0719 \\
1.0632\end{array}$ & $\begin{array}{l}.15358 \\
.15131 \\
.14903 \\
.14475 \\
.14448 \\
.14220 \\
.13993 \\
.13746 \\
.13539 \\
.13313\end{array}$ & $\begin{array}{l}1871.3 \\
1796.8 \\
1726.5 \\
1560.2 \\
1597.5 \\
1538.3 \\
1482.2 \\
1429.1 \\
1378.8 \\
1331.0\end{array}$ & $\begin{array}{l}.000837 \\
.000830 \\
.000824 \\
.000817 \\
.000809 \\
.000802 \\
.000794 \\
.000787 \\
.000779 \\
.000771\end{array}$ & $\begin{array}{l}102 . \\
104 . \\
106 . \\
108 . \\
110 . \\
112 . \\
114 . \\
116 . \\
118 . \\
120 .\end{array}$ & $\begin{array}{l}1.1419 \\
1.1336 \\
1.1253 \\
1.1169 \\
1.1086 \\
1.1001 \\
1.0916 \\
1.0831 \\
1.0745 \\
1.0559\end{array}$ & $\begin{array}{l}.15428 \\
.15202 \\
.14976 \\
.14750 \\
.14524 \\
.14298 \\
.14072 \\
.13847 \\
.13622 \\
.13397\end{array}$ & $\begin{array}{l}1886.4 \\
1811.4 \\
1740.8 \\
1674.1 \\
1611.1 \\
1551.6 \\
1495.2 \\
1441.9 \\
1391.3 \\
1343.3\end{array}$ & $\begin{array}{l}.000841 \\
.000835 \\
.000828 \\
.000821 \\
.000814 \\
.000807 \\
.000799 \\
.000792 \\
.000784 \\
.000776\end{array}$ \\
\hline 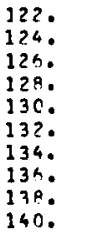 & $\begin{array}{l}1.0544 \\
1.0456 \\
1.0367 \\
1.0277 \\
1.0187 \\
1.0095 \\
1.0004 \\
.0911 \\
.0919 \\
.0723\end{array}$ & $\begin{array}{l}.13 C 97 \\
.12861 \\
.12637 \\
.12413 \\
.12190 \\
.11968 \\
.11748 \\
.11529 \\
.11112 \\
.11096\end{array}$ & $\begin{array}{r}1285.6 \\
1242.4 \\
1201.3 \\
1162.2 \\
1124.9 \\
1089.3 \\
1055.3 \\
1022.8 \\
991.7 \\
961.9\end{array}$ & $\begin{array}{l}.000752 \\
.000754 \\
.000745 \\
.000737 \\
.000728 \\
.000719 \\
.000710 \\
.000701 \\
.000692 \\
.000683\end{array}$ & $\begin{array}{l}122 . \\
1240^{\circ} \\
1260^{\circ} \\
1280^{\circ} \\
1300^{\circ} \\
1320^{\circ} \\
1340^{\circ} \\
130^{\circ} \\
1380^{\circ} \\
140 .\end{array}$ & $\begin{array}{r}1.0572 \\
1.0485 \\
1.0397 \\
1.0308 \\
1.0219 \\
1.0129 \\
1.0039 \\
.9947 \\
.9855 \\
.9763\end{array}$ & $\begin{array}{l}.13173 \\
.12949 \\
.12727 \\
.12505 \\
.12284 \\
.12064 \\
.11846 \\
.11629 \\
.11414 \\
.111200\end{array}$ & $\begin{array}{r}1297.7 \\
1234.3 \\
1213.1 \\
1173.8 \\
1136.4 \\
1100.7 \\
1066.6 \\
1034.0 \\
1002.9 \\
973.1\end{array}$ & $\begin{array}{l}.000768 \\
.000760 \\
.000751 \\
.000743 \\
.000734 \\
.000726 \\
.000717 \\
.000708 \\
.000700 \\
.000691\end{array}$ \\
\hline $\begin{array}{l}142 . \\
1440 \\
140^{\circ} \\
148 . \\
150^{\circ} \\
152 . \\
1540^{\circ} \\
150^{\circ} \\
158 . \\
160 .\end{array}$ & $\begin{array}{l}.9329 \\
.9532 \\
.9435 \\
.9338 \\
.0239 \\
.9139 \\
.9039 \\
.8937 \\
.8835 \\
.8732\end{array}$ & $\begin{array}{l}.10883 \\
.10672 \\
.10464 \\
.10258 \\
.10057 \\
.09961 \\
.09671 \\
.09476 \\
.09779 \\
.09087\end{array}$ & $\begin{array}{l}933.4 \\
906.1 \\
879.9 \\
854.7 \\
830.5 \\
807.3 \\
784.9 \\
763.4 \\
742.7 \\
722.7\end{array}$ & $\begin{array}{l}.000674 \\
.000665 \\
.000656 \\
.000647 \\
.000639 \\
.000630 \\
.000623 \\
.000614 \\
.000606 \\
.000597\end{array}$ & $\begin{array}{l}142 . \\
144 . \\
146 . \\
148 . \\
150 . \\
152 . \\
1540^{\circ} \\
156^{\circ} \\
158^{\circ} \\
160 .\end{array}$ & $\begin{array}{l}.9669 \\
.9575 \\
.9480 \\
.9384 \\
.9287 \\
.9189 \\
.9091 \\
.8992 \\
.8891 \\
.8791\end{array}$ & $\begin{array}{l}.10989 \\
.10780 \\
.10573 \\
.10370 \\
.10170 \\
.09976 \\
.09787 \\
.09594 \\
.09400 \\
.09209\end{array}$ & $\begin{array}{l}944.5 \\
917.1 \\
890.9 \\
865.7 \\
841.5 \\
818.2 \\
795.9 \\
774.4 \\
753.7 \\
733.8\end{array}$ & $\begin{array}{l}.000682 \\
.000673 \\
.000665 \\
.000656 \\
.000648 \\
.000639 \\
.000632 \\
.000624 \\
.000615 \\
.000607\end{array}$ \\
\hline $\begin{array}{l}165 . \\
170 . \\
175 \circ \\
180 . \\
185 . \\
190 . \\
195 . \\
200 . \\
210 . \\
220 .\end{array}$ & $\begin{array}{l}.8470 \\
.8203 \\
.7932 \\
.7657 \\
.7381 \\
.7104 \\
.6829 \\
.6558 \\
.6037 \\
.5558\end{array}$ & $\begin{array}{l}.08623 \\
.08194 \\
.07771 \\
.07387 \\
.07033 \\
.06709 \\
.06415 \\
.06151 \\
.05702 \\
.05343\end{array}$ & $\begin{array}{l}676.0 \\
633.5 \\
594.6 \\
559.2 \\
527.0 \\
497.7 \\
471.2 \\
447.4 \\
407.3 \\
376.1\end{array}$ & $\begin{array}{l}.000578 \\
.000560 \\
.000545 \\
.000532 \\
.000522 \\
.000515 \\
.000511 \\
.000512 \\
.000554 \\
.000551\end{array}$ & $\begin{array}{l}165 . \\
170 . \\
175 . \\
180 . \\
185 . \\
190 . \\
195 . \\
200 . \\
210 . \\
220 .\end{array}$ & $\begin{array}{r}.8535 \\
.8275 \\
.8012 \\
.7745 \\
.7477 \\
.7209 \\
.6942 \\
.6679 \\
.6171 \\
.5700\end{array}$ & $\begin{array}{l}.08750 \\
.08315 \\
.07905 \\
.07524 \\
.07171 \\
.06847 \\
.06552 \\
.06286 \\
.05833 \\
.05469\end{array}$ & $\begin{array}{l}687.2 \\
644.7 \\
606.0 \\
570.7 \\
538.5 \\
509.3 \\
482.8 \\
459.0 \\
418.5 \\
386.6\end{array}$ & $\begin{array}{l}.000588 \\
.000571 \\
.000556 \\
.000543 \\
.000533 \\
.000526 \\
.000522 \\
.000521 \\
.000531 \\
.000555\end{array}$ \\
\hline $\begin{array}{l}230 . \\
240 . \\
250 . \\
260 . \\
270 . \\
280 . \\
290 . \\
300 . \\
310 . \\
320 .\end{array}$ & $\begin{array}{l}.5129 \\
.4754 \\
.4428 \\
.4146 \\
.3900 \\
.3685 \\
.3495 \\
.3327 \\
.3176 \\
.3041\end{array}$ & $\begin{array}{l}.05056 \\
.04826 \\
.04643 \\
.04499 \\
.04388 \\
.04305 \\
.04243 \\
.04199 \\
.04177 \\
.04190\end{array}$ & $\begin{array}{l}352.4 \\
334.7 \\
321.7 \\
312.3 \\
305.5 \\
300.6 \\
297.6 \\
295.6 \\
294.5 \\
294.3\end{array}$ & $\begin{array}{l}.000590 \\
.000637 \\
.000690 \\
.000747 \\
.000807 \\
.000868 \\
.000931 \\
.000994 \\
.001061 \\
.001134\end{array}$ & $\begin{array}{l}230 . \\
240 . \\
250 . \\
260 . \\
270 . \\
280 . \\
290 . \\
300 . \\
310 . \\
320 .\end{array}$ & $\begin{array}{l}.5275 \\
.4900 \\
.4371 \\
.4284 \\
.4033 \\
.3813 \\
.3618 \\
.3445 \\
.3290 \\
.3150\end{array}$ & $\begin{array}{l}.05176 \\
.04941 \\
.04752 \\
.04601 \\
.04484 \\
.04393 \\
.04325 \\
.04274 \\
.04247 \\
.04255\end{array}$ & $\begin{array}{l}362.2 \\
343.7 \\
329.9 \\
319.6 \\
312.2 \\
306.9 \\
303.2 \\
300.7 \\
299.3 \\
298.7\end{array}$ & $\begin{array}{l}.000590 \\
.000634 \\
.000684 \\
.000737 \\
.000794 \\
.000852 \\
.000912 \\
.000972 \\
.001035 \\
.001106\end{array}$ \\
\hline
\end{tabular}

26.0 MP: I sobar 
Tatle 5. Transoort Prodertles of Oxyoen. Isobars, SI Units.

27.C MP. Isobar

28.0 MPs Isobser

\begin{tabular}{|c|c|c|c|c|c|c|c|c|c|}
\hline $\begin{array}{c}\text { Temp. } \\
K\end{array}$ & $\begin{array}{l}\text { Density } \\
\text { g/cm* } * 3\end{array}$ & $\begin{array}{l}\text { Thermal } \\
\text { Cond. } \\
\text { w/mek }\end{array}$ & $\begin{array}{c}\text { VIscosity } \\
m / e r o- \\
\text { o/cmos }\end{array}$ & $\begin{array}{c}\text { Thermal } \\
\text { Diffusivity } \\
\text { em*21s }\end{array}$ & $\begin{array}{c}\text { Teno. } \\
k\end{array}$ & $\begin{array}{l}\text { Density } \\
\text { g/cm* } * 3\end{array}$ & $\begin{array}{l}\text { Thermal } \\
\text { Cond. } \\
\text { Hom.k }\end{array}$ & $\begin{array}{c}\text { VIscosity } \\
\text { mlero- } \\
\text { olemos }\end{array}$ & $\begin{array}{c}\text { Thermol } \\
\text { olffusivity } \\
\text { cm*2*2is }\end{array}$ \\
\hline $\begin{array}{l}57.390 \\
58 . \\
6 C .\end{array}$ & $\begin{array}{l}1.3228 \\
1.3203 \\
1.3122\end{array}$ & $\begin{array}{l}.21007 \\
.20006 \\
.20583\end{array}$ & $\begin{array}{l}5941.2 \\
5554.2 \\
5271.5\end{array}$ & $\begin{array}{l}.000962 \\
.000960 \\
.000953\end{array}$ & $\begin{array}{l}* \quad 57.509 \\
58 . \\
30 .\end{array}$ & $\begin{array}{l}1.3234 \\
1.3214 \\
1.3133\end{array}$ & $\begin{array}{l}.21031 \\
.20949 \\
.20627\end{array}$ & $\begin{array}{l}5668.2 \\
5596.4 \\
5310.8\end{array}$ & $\begin{array}{l}.000963 \\
.000961 \\
.000955\end{array}$ \\
\hline $\begin{array}{l}62 . \\
64 . \\
66 . \\
68 . \\
70 . \\
72 . \\
74 . \\
76 . \\
78 . \\
80 .\end{array}$ & $\begin{array}{l}1.3041 \\
1.2961 \\
1.2881 \\
1.2802 \\
1.2722 \\
1.2643 \\
1.2564 \\
1.2484 \\
1.2405 \\
1.2325\end{array}$ & $\begin{array}{l}.20279 \\
.19989 \\
.19712 \\
.19445 \\
.19187 \\
.18936 \\
.18691 \\
.19451 \\
.18215 \\
.17981\end{array}$ & $\begin{array}{l}5000.3 \\
4741.8 \\
4695.9 \\
4269.3 \\
4043.8 \\
3837.0 \\
3442.8 \\
3460.0 \\
3288.8 \\
3128.2\end{array}$ & $\begin{array}{l}.000047 \\
.000942 \\
.000936 \\
.000932 \\
.000927 \\
.000922 \\
.000918 \\
.000913 \\
.000908 \\
.000904\end{array}$ & $\begin{array}{l}62 . \\
64 . \\
66 . \\
38 . \\
70 . \\
72 . \\
74 . \\
76 . \\
78 . \\
80 .\end{array}$ & $\begin{array}{l}1.3052 \\
1.2972 \\
1.2892 \\
1.2813 \\
1.2734 \\
1.2655 \\
1.2576 \\
1.2497 \\
1.2418 \\
1.2339\end{array}$ & $\begin{array}{l}.20323 \\
.20034 \\
.19757 \\
.19491 \\
.19234 \\
.18984 \\
.18741 \\
.18502 \\
.18267 \\
.18035\end{array}$ & $\begin{array}{l}5037.1 \\
4776.1 \\
4528.3 \\
4293.9 \\
4072.7 \\
3864.4 \\
3668.6 \\
3484.7 \\
3312.3 \\
3150.6\end{array}$ & $\begin{array}{l}.000949 \\
.000943 \\
.000939 \\
.000933 \\
.000929 \\
.000924 \\
.000920 \\
.000915 \\
.000911 \\
.000906\end{array}$ \\
\hline $\begin{array}{l}82 . \\
84 . \\
880 \\
89 . \\
90 . \\
920 \\
940 \\
96 . \\
90^{\circ} \\
100 .\end{array}$ & $\begin{array}{l}1.2246 \\
1.2166 \\
1.2086 \\
1.2006 \\
1.1925 \\
1.1845 \\
1.1744 \\
1.1583 \\
1.1602 \\
1.1520\end{array}$ & $\begin{array}{l}.17751 \\
.17522 \\
.17295 \\
.17069 \\
.16843 \\
.16618 \\
.16394 \\
.16170 \\
.15945 \\
.15721\end{array}$ & $\begin{array}{l}2977.7 \\
2836.6 \\
2704.4 \\
2580.4 \\
2454.2 \\
2355.2 \\
2252.8 \\
2156.7 \\
2036.3 \\
1981.3\end{array}$ & $\begin{array}{l}.000809 \\
.000894 \\
.000889 \\
.000884 \\
.000879 \\
.000874 \\
.000868 \\
.000883 \\
.000857 \\
.000851\end{array}$ & $\begin{array}{l}82 . \\
84 . \\
86 . \\
88 . \\
90 . \\
92 . \\
94 . \\
93 . \\
98 . \\
100 .\end{array}$ & $\begin{array}{l}1.2260 \\
1.2180 \\
1.2101 \\
1.2021 \\
1.1941 \\
1.1861 \\
1.1781 \\
1.1701 \\
1.1620 \\
1.1539\end{array}$ & $\begin{array}{l}.17805 \\
.17578 \\
.17352 \\
.17127 \\
.16903 \\
.16680 \\
.16457 \\
.16234 \\
.16011 \\
.15786\end{array}$ & $\begin{array}{l}2999.1 \\
2857.2 \\
2724.1 \\
2599.4 \\
2482.4 \\
2372.7 \\
2269.8 \\
2173.1 \\
2082.2 \\
1996.7\end{array}$ & $\begin{array}{l}.000902 \\
.000897 \\
.000892 \\
.000887 \\
.000882 \\
.000877 \\
.000872 \\
.000866 \\
.000861 \\
.000855\end{array}$ \\
\hline $\begin{array}{l}102 . \\
104 . \\
1060 \\
1080 \\
110 . \\
112 . \\
1140 \\
116 . \\
110 . \\
120 .\end{array}$ & $\begin{array}{l}1.1439 \\
1.1356 \\
1.1274 \\
1.1191 \\
1.1108 \\
1.1024 \\
1.0940 \\
1.0856 \\
1.0771 \\
1.0686\end{array}$ & $\begin{array}{l}.15497 \\
.15272 \\
.15048 \\
.14824 \\
.14599 \\
.14375 \\
.14151 \\
.13927 \\
.13704 \\
.13481\end{array}$ & $\begin{array}{l}1901.4 \\
1826.0 \\
1755.0 \\
1687.9 \\
1624.6 \\
1564.8 \\
1508.2 \\
1454.5 \\
1403.7 \\
1355.5\end{array}$ & $\begin{array}{l}.000845 \\
.000839 \\
.000832 \\
.000825 \\
.000818 \\
.000811 \\
.000804 \\
.000797 \\
.000789 \\
.000781\end{array}$ & $\begin{array}{l}102 . \\
104 . \\
105 . \\
108 . \\
110 . \\
112 . \\
114 . \\
115 . \\
118 . \\
120 .\end{array}$ & $\begin{array}{l}1.1458 \\
1.1376 \\
1.1295 \\
1.1212 \\
1.1130 \\
1.1047 \\
1.0964 \\
1.0880 \\
1.0796 \\
1.0712\end{array}$ & $\begin{array}{l}.15565 \\
.15342 \\
.15120 \\
.14897 \\
.14674 \\
.14451 \\
.14229 \\
.14006 \\
.13785 \\
.13563\end{array}$ & $\begin{array}{l}1916.3 \\
1840.5 \\
1769.1 \\
1701.7 \\
1630.1 \\
1577.9 \\
1521.0 \\
1467.1 \\
1416.1 \\
1367.6\end{array}$ & $\begin{array}{l}.000849 \\
.000842 \\
.000836 \\
.000830 \\
.000823 \\
.0008116 \\
.000809 \\
.000801 \\
.000794 \\
.000786\end{array}$ \\
\hline $\begin{array}{l}122 . \\
1240^{\circ} \\
1260^{\circ} \\
128 . \\
1300^{\circ} \\
1320^{\circ} \\
1340^{\circ} \\
1360^{\circ} \\
1380^{\circ} \\
140 .\end{array}$ & $\begin{array}{l}1.0600 \\
1.0513 \\
1.0426 \\
1.0330 \\
1.0251 \\
1.0162 \\
1.0073 \\
.9983 \\
.9892 \\
.9801\end{array}$ & $\begin{array}{l}.13258 \\
.13036 \\
.12815 \\
.12595 \\
.12376 \\
.12159 \\
.11942 \\
.11727 \\
.11514 \\
.11302\end{array}$ & $\begin{array}{r}1309.7 \\
1266.2 \\
1224.8 \\
1185.4 \\
1147.8 \\
1112.0 \\
1077.8 \\
1045.1 \\
1013.9 \\
984.0\end{array}$ & $\begin{array}{l}.000773 \\
.000765 \\
.000757 \\
.000749 \\
.000741 \\
.000732 \\
.000724 \\
.000715 \\
.000707 \\
.000698\end{array}$ & $\begin{array}{l}122 . \\
1240^{\circ} \\
1260^{\circ} \\
1280^{\circ} \\
1300^{\circ} \\
1320^{\circ} \\
1340^{\circ} \\
1360^{\circ} \\
1380^{\circ} \\
140 .\end{array}$ & $\begin{array}{r}1.0627 \\
1.0541 \\
1.0455 \\
1.0369 \\
1.0282 \\
1.0194 \\
1.0108 \\
1.0017 \\
.9928 \\
.9838\end{array}$ & $\begin{array}{l}.13342 \\
.13122 \\
.12903 \\
.12083 \\
.12467 \\
.12251 \\
.12037 \\
.11023 \\
.11612 \\
.11402\end{array}$ & $\begin{array}{r}1321.6 \\
1277.9 \\
1236.4 \\
1196.8 \\
1159.1 \\
1123.2 \\
1086.9 \\
1056.1 \\
1024.8 \\
994.8\end{array}$ & $\begin{array}{l}.000779 \\
.000771 \\
.000763 \\
.000755 \\
.000747 \\
.000739 \\
.000730 \\
.000722 \\
.000714 \\
.000705\end{array}$ \\
\hline $\begin{array}{l}142 . \\
144 . \\
148 . \\
1480 \\
150 . \\
152 . \\
1540 \\
156 . \\
158 . \\
160 .\end{array}$ & $\begin{array}{l}.9709 \\
.9316 \\
.9522 \\
.9428 \\
.9333 \\
.9238 \\
.9141 \\
.9044 \\
.8946 \\
.8847\end{array}$ & $\begin{array}{l}.11093 \\
.10895 \\
.10691 \\
.10679 \\
.10282 \\
.10088 \\
.09901 \\
.09709 \\
.09517 \\
.09328\end{array}$ & $\begin{array}{l}955.4 \\
928.0 \\
901.7 \\
876.5 \\
852.3 \\
829.0 \\
898.6 \\
785.2 \\
764.5 \\
744.6\end{array}$ & $\begin{array}{l}.000690 \\
.000681 \\
.000673 \\
.000664 \\
.000656 \\
.000648 \\
.000641 \\
.000633 \\
.000625 \\
.000617\end{array}$ & $\begin{array}{l}142 . \\
144 . \\
146 . \\
148 . \\
150 . \\
152 . \\
154 . \\
156 . \\
150^{\circ} \\
160 .\end{array}$ & $\begin{array}{r}.9747 \\
.9656 \\
.9564 \\
.9471 \\
.9378 \\
.9284 \\
.9190 \\
.9094 \\
.8998 \\
.8902\end{array}$ & $\begin{array}{l}.11195 \\
.10989 \\
.10786 \\
.10587 \\
.10390 \\
.10198 \\
.10012 \\
.09822 \\
.09632 \\
.09445\end{array}$ & $\begin{array}{l}966.1 \\
938.7 \\
912.3 \\
887.1 \\
862.8 \\
839.6 \\
817.2 \\
795.7 \\
775.0 \\
755.1\end{array}$ & $\begin{array}{l}.000697 \\
.000689 \\
.000680 \\
.000672 \\
.000664 \\
.000657 \\
.000649 \\
.000641 \\
.000634 \\
.000626\end{array}$ \\
\hline $\begin{array}{l}165 . \\
170 . \\
175 . \\
180 . \\
185 . \\
190 . \\
195 . \\
200 . \\
210 . \\
220 .\end{array}$ & $\begin{array}{l}.8598 \\
.8344 \\
.8087 \\
.7828 \\
.7568 \\
.7307 \\
.7064 \\
.6792 \\
.6297 \\
.5834\end{array}$ & $\begin{array}{l}.08873 \\
.08442 \\
.08036 \\
.07657 \\
.07305 \\
.06981 \\
.06686 \\
.06419 \\
.05961 \\
.05592\end{array}$ & $\begin{array}{l}698.0 \\
655.6 \\
617.0 \\
581.7 \\
549.6 \\
520.5 \\
494.0 \\
470.1 \\
429.3 \\
396.9\end{array}$ & $\begin{array}{l}.000598 \\
.000582 \\
.000567 \\
.000554 \\
.000544 \\
.000536 \\
.000532 \\
.000531 \\
.000539 \\
.000550\end{array}$ & $\begin{array}{l}165 . \\
170 . \\
175 . \\
180 . \\
185 . \\
190 . \\
195 . \\
200 . \\
210 . \\
220 .\end{array}$ & $\begin{array}{r}.8658 \\
.8410 \\
.8159 \\
.7907 \\
.7653 \\
.7400 \\
.7148 \\
.6899 \\
.6415 \\
.5961\end{array}$ & $\begin{array}{l}.08994 \\
.08567 \\
.08163 \\
.07786 \\
.07436 \\
.07113 \\
.06817 \\
.06548 \\
.06086 \\
.05712\end{array}$ & $\begin{array}{l}708.6 \\
666.2 \\
627.6 \\
592.4 \\
560.4 \\
531.2 \\
504.8 \\
480.8 \\
439.7 \\
406.9\end{array}$ & $\begin{array}{l}.000508 \\
.000591 \\
.000577 \\
.000564 \\
.000554 \\
.000547 \\
.000542 \\
.000540 \\
.000547 \\
.000565\end{array}$ \\
\hline $\begin{array}{l}230 . \\
240 . \\
250 . \\
260 . \\
270 . \\
280 . \\
290 . \\
300 . \\
310 . \\
320 .\end{array}$ & $\begin{array}{l}.5414 \\
.5039 \\
.4708 \\
.4418 \\
.4163 \\
.3938 \\
.3738 \\
.3560 \\
.3401 \\
.3257\end{array}$ & $\begin{array}{l}.05294 \\
.05053 \\
.04858 \\
.04702 \\
.04578 \\
.04481 \\
.04406 \\
.04356 \\
.04317 \\
.04321\end{array}$ & $\begin{array}{l}371.7 \\
352.5 \\
337.9 \\
327.0 \\
318.9 \\
313.0 \\
308.8 \\
305.9 \\
304.1 \\
303.2\end{array}$ & $\begin{array}{l}.00059 ? \\
.00063 ? \\
.000479 \\
.000729 \\
.000783 \\
.000838 \\
.000895 \\
.000953 \\
.001013 \\
.001081\end{array}$ & $\begin{array}{l}230 . \\
240 . \\
250 . \\
260 . \\
270 . \\
280 . \\
290 . \\
300 . \\
310 . \\
320 .\end{array}$ & $\begin{array}{l}.5545 \\
.5172 \\
.4840 \\
.4547 \\
.4288 \\
.4059 \\
.3855 \\
.3673 \\
.3510 \\
.3362\end{array}$ & $\begin{array}{l}.05409 \\
.05163 \\
.04964 \\
.04602 \\
.04672 \\
.04568 \\
.04487 \\
.04425 \\
.04387 \\
.04387\end{array}$ & $\begin{array}{l}381.1 \\
361.1 \\
345.8 \\
334.2 \\
325.5 \\
319.1 \\
314.4 \\
311.1 \\
301.9 \\
307.7\end{array}$ & $\begin{array}{l}.000594 \\
.000631 \\
.000675 \\
.000723 \\
.000773 \\
.000826 \\
.000880 \\
.000935 \\
.000993 \\
.001058\end{array}$ \\
\hline
\end{tabular}

* Two Phase Boundary 
Table 5. Transport prooertles of cxygen, Isobars, si Units.

29.0 MPa Isohar

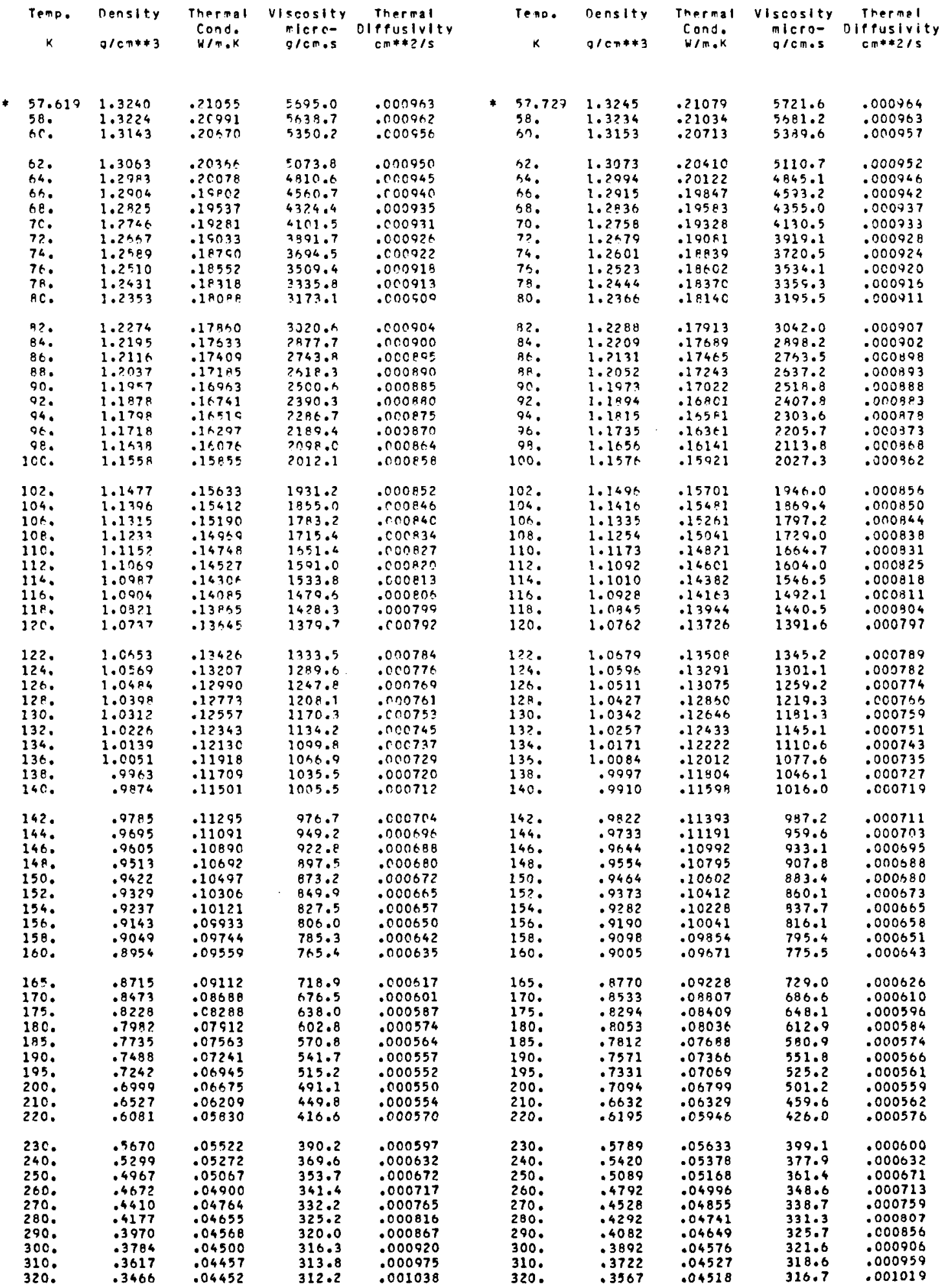

Thermal viscosity Thermel
Cond. micro- Diffusivity
wimek

$30.0 \mathrm{MPa}$ IsODar

(n) 
Table 5. Transport Properties of Oxyaen. Isooars, SI Unlts.

35.0 MPE Isobar

40.0 MPQ Isobar

\begin{tabular}{|c|c|c|c|c|c|c|c|c|c|}
\hline $\begin{array}{c}\text { Temp. } \\
k\end{array}$ & $\begin{array}{l}\text { Density } \\
\text { g/en* } \neq=3\end{array}$ & $\begin{array}{l}\text { Thermal } \\
\text { Cond. } \\
\text { w/mok }\end{array}$ & $\begin{array}{c}\text { VIscosity } \\
\text { micro- } \\
\text { o/emos }\end{array}$ & $\begin{array}{c}\text { Thermal } \\
\text { Diffusivity } \\
\text { cm } * 2 / s\end{array}$ & Temp. & $\begin{array}{l}\text { Denslty } \\
\text { g/cm**3 }\end{array}$ & $\begin{array}{l}\text { Thermol } \\
\text { Cond. } \\
\text { Wrmek }\end{array}$ & $\begin{array}{c}\text { VIscosity } \\
\text { micro- } \\
\text { g/cmos }\end{array}$ & $\begin{array}{c}\text { Thermal } \\
\text { oiffusivity } \\
\text { cm* } \neq 2 / s\end{array}$ \\
\hline $\begin{array}{l}62 . \\
64 . \\
66 . \\
68 . \\
70 . \\
72 . \\
74 . \\
76 . \\
78 . \\
80 .\end{array}$ & $\begin{array}{l}1.3126 \\
1.3047 \\
1.2939 \\
1.2892 \\
1.2915 \\
1.2738 \\
1.2662 \\
1.2585 \\
1.2509 \\
1.2432\end{array}$ & $\begin{array}{l}.20625 \\
.20340 \\
.20069 \\
.19210 \\
.19559 \\
.19317 \\
.19080 \\
.18349 \\
.18622 \\
.18399\end{array}$ & $\begin{array}{l}5296.2 \\
5018.7 \\
4756.2 \\
4508.5 \\
4275.3 \\
4056.1 \\
3850.4 \\
3657.5 \\
3476.8 \\
3307.5\end{array}$ & $\begin{array}{l}.000959 \\
.000954 \\
.000950 \\
.000946 \\
.000942 \\
.000938 \\
.000934 \\
.000931 \\
.000927 \\
.000923\end{array}$ & $\begin{array}{l}62 . \\
64: \\
66 . \\
68 . \\
70 . \\
72 . \\
74 . \\
76 . \\
78 . \\
80 .\end{array}$ & $\begin{array}{l}1.3176 \\
1.3099 \\
1.3022 \\
1.2946 \\
1.2871 \\
1.2795 \\
1.2720 \\
1.2645 \\
1.2570 \\
1.2496\end{array}$ & $\begin{array}{l}.20835 \\
.20554 \\
.20287 \\
.20031 \\
.19786 \\
.19548 \\
.19316 \\
.19090 \\
.18869 \\
.18651\end{array}$ & $\begin{array}{l}5483.5 \\
5193.7 \\
4920.3 \\
4662.7 \\
4420.7 \\
4193.5 \\
3980.5 \\
3780.9 \\
3594.0 \\
3419.2\end{array}$ & $\begin{array}{l}.000965 \\
.000961 \\
.000957 \\
.000954 \\
.000951 \\
.000947 \\
.000944 \\
.000741 \\
.000938 \\
.000934\end{array}$ \\
\hline $\begin{array}{l}102 . \\
104 . \\
106 . \\
108 \\
110 . \\
112 . \\
114 . \\
116 . \\
118 . \\
120 .\end{array}$ & $\begin{array}{l}1.1587 \\
1.1510 \\
1.1432 \\
1.1354 \\
1.1273 \\
1.1198 \\
1.1119 \\
1.1041 \\
1.0962 \\
1.0882\end{array}$ & $\begin{array}{l}.16030 \\
.15817 \\
.15603 \\
.15390 \\
.15177 \\
.14964 \\
.14752 \\
.14540 \\
.14328 \\
.14117\end{array}$ & $\begin{array}{l}2019.3 \\
1940.8 \\
1866.3 \\
1706.3 \\
1730.3 \\
1367.9 \\
1608.9 \\
1553.1 \\
1500.3 \\
1450.2\end{array}$ & $\begin{array}{l}.000874 \\
.000860 \\
.000843 \\
.000857 \\
.000852 \\
.000846 \\
.000840 \\
.000833 \\
.000827 \\
.000820\end{array}$ & $\begin{array}{l}102 . \\
104 . \\
106 . \\
10 \% . \\
110 . \\
112 . \\
114 . \\
116 . \\
118 . \\
120 .\end{array}$ & $\begin{array}{l}1.1674 \\
1.1599 \\
1.1523 \\
1.1448 \\
1.1373 \\
1.1297 \\
1.1222 \\
1.1146 \\
1.1070 \\
1.0994\end{array}$ & $\begin{array}{l}.15347 \\
.15140 \\
.15933 \\
.15726 \\
.15519 \\
.15312 \\
.15106 \\
.14900 \\
.14594 \\
.14489\end{array}$ & $\begin{array}{l}2091.3 \\
2010.5 \\
1934.2 \\
1852.3 \\
1794.4 \\
1730.4 \\
1659.8 \\
1512.6 \\
1558.4 \\
1507.0\end{array}$ & $\begin{array}{l}.000891 \\
.000886 \\
.000861 \\
.000875 \\
.000870 \\
.000855 \\
.000859 \\
.000854 \\
.000948 \\
.00084 ?\end{array}$ \\
\hline $\begin{array}{l}142 . \\
1440 \\
146 . \\
148 \\
150 \\
1520^{\circ} \\
154 . \\
156 . \\
158 . \\
160 .\end{array}$ & $\begin{array}{l}.9903 \\
.9910 \\
.9827 \\
.9743 \\
.9659 \\
.9575 \\
.0401 \\
.0406 \\
.9321 \\
.9235\end{array}$ & $\begin{array}{l}.11833 \\
.11638 \\
.11476 \\
.11296 \\
.11099 \\
.10925 \\
.10736 \\
.10555 \\
.10375 \\
.10199\end{array}$ & $\begin{array}{r}1037.4 \\
1009.4 \\
982.5 \\
956.5 \\
932.2 \\
908.6 \\
885.9 \\
864 . ? \\
843.3 \\
623 . ?\end{array}$ & $\begin{array}{l}.000744 \\
.000736 \\
.000720 \\
.000722 \\
.000715 \\
.000709 \\
.000702 \\
.000696 \\
.000689 \\
.000682\end{array}$ & $\begin{array}{l}142 . \\
144 . \\
146 . \\
149 . \\
150 . \\
152 . \\
154 . \\
154 . \\
154 . \\
150 .\end{array}$ & $\begin{array}{r}1.0146 \\
1.0068 \\
.9970 \\
.9911 \\
.9932 \\
.9753 \\
.9674 \\
.9595 \\
.9515 \\
.9436\end{array}$ & $\begin{array}{l}.12302 \\
.12113 \\
.11926 \\
.11741 \\
.11559 \\
.11381 \\
.11205 \\
.11030 \\
.10054 \\
.10684\end{array}$ & $\begin{array}{r}1084.9 \\
1053.4 \\
1029.1 \\
1002.9 \\
977.9 \\
953.9 \\
930.9 \\
908.8 \\
887.6 \\
957.2\end{array}$ & $\begin{array}{l}.000772 \\
.000763 \\
.000760 \\
.000753 \\
.000747 \\
.000741 \\
.000735 \\
.000729 \\
.000723 \\
.000717\end{array}$ \\
\hline $\begin{array}{l}165 . \\
1700^{\circ} \\
1750^{\circ} \\
180^{\circ} \\
1850^{\circ} \\
190 . \\
195 \\
200 \\
210 . \\
220 .\end{array}$ & $\begin{array}{l}.9020 \\
.8804 \\
.9587 \\
.8369 \\
.8152 \\
.7935 \\
.7719 \\
.7505 \\
.7087 \\
.0687\end{array}$ & $\begin{array}{l}.09771 \\
.09354 \\
.08978 \\
.08513 \\
.08271 \\
.07952 \\
.07656 \\
.07392 \\
.06899 \\
.04498\end{array}$ & $\begin{array}{l}776.3 \\
733.8 \\
695.0 \\
659.8 \\
527.6 \\
598.2 \\
571.5 \\
547.1 \\
504.6 \\
469.5\end{array}$ & $\begin{array}{l}.000647 \\
.000553 \\
.000640 \\
.000628 \\
.000618 \\
.000611 \\
.000505 \\
.000601 \\
.000500 \\
.000407\end{array}$ & $\begin{array}{l}155^{\circ} \\
170 . \\
175 . \\
180 . \\
185 . \\
190 . \\
195 . \\
200^{\circ} \\
210 . \\
220 .\end{array}$ & $\begin{array}{l}.9234 \\
.9035 \\
.8835 \\
.8634 \\
.8433 \\
.8234 \\
.8036 \\
.7840 \\
.7455 \\
.7084\end{array}$ & $\begin{array}{l}.10269 \\
.09873 \\
.09496 \\
.09138 \\
.08802 \\
.09486 \\
.08190 \\
.07915 \\
.07424 \\
.07007\end{array}$ & $\begin{array}{l}919.8 \\
776.7 \\
737.5 \\
701.8 \\
539.3 \\
639.8 \\
612.5 \\
587.7 \\
544.2 \\
508.0\end{array}$ & $\begin{array}{l}.000702 \\
.000539 \\
.000577 \\
.000565 \\
.000557 \\
.000549 \\
.000643 \\
.000539 \\
.000535 \\
.000637\end{array}$ \\
\hline $\begin{array}{l}230 . \\
240 . \\
250 . \\
260 . \\
270 . \\
280 . \\
290 . \\
300 . \\
310 . \\
320 .\end{array}$ & $\begin{array}{l}.6308 \\
.5956 \\
.5537 \\
.5335 \\
.5054 \\
.4221 \\
.4599 \\
.4397 \\
.4213 \\
.4046\end{array}$ & $\begin{array}{l}.06162 \\
.05884 \\
.05653 \\
.05459 \\
.05298 \\
.05159 \\
.05046 \\
.04548 \\
.04878 \\
.04848\end{array}$ & $\begin{array}{l}440.7 \\
417.2 \\
398.2 \\
382.9 \\
370.7 \\
361.0 \\
353.4 \\
347.4 \\
342.0 \\
339.4\end{array}$ & $\begin{array}{l}.000722 \\
.000444 \\
.000672 \\
.000704 \\
.000740 \\
.000778 \\
.000817 \\
.000858 \\
.0009 C 1 \\
.000951\end{array}$ & $\begin{array}{l}230 . \\
240 . \\
250 . \\
250 . \\
270 . \\
280 . \\
270 . \\
300 . \\
320 . \\
320 .\end{array}$ & $\begin{array}{l}.6730 \\
.6396 \\
.6084 \\
.5794 \\
.5526 \\
.5780 \\
.5053 \\
.4845 \\
.4653 \\
.4477\end{array}$ & $\begin{array}{l}.06654 \\
.05358 \\
.06107 \\
.05895 \\
.05714 \\
.05558 \\
.05424 \\
.05309 \\
.05218 \\
.05147\end{array}$ & $\begin{array}{l}477.8 \\
452.7 \\
432.0 \\
414.9 \\
400.9 \\
389.4 \\
380.1 \\
372.6 \\
366.6 \\
351.8\end{array}$ & $\begin{array}{l}.000647 \\
.000663 \\
.000083 \\
.000708 \\
.000735 \\
.000757 \\
.000799 \\
.000833 \\
.000468 \\
.000909\end{array}$ \\
\hline
\end{tabular}

* Two Phase poundory 
Toble 5. Transport propertles of Oxygen, Isobars, SI UnIts.

$45.0 \mathrm{MPa}$ Isobar

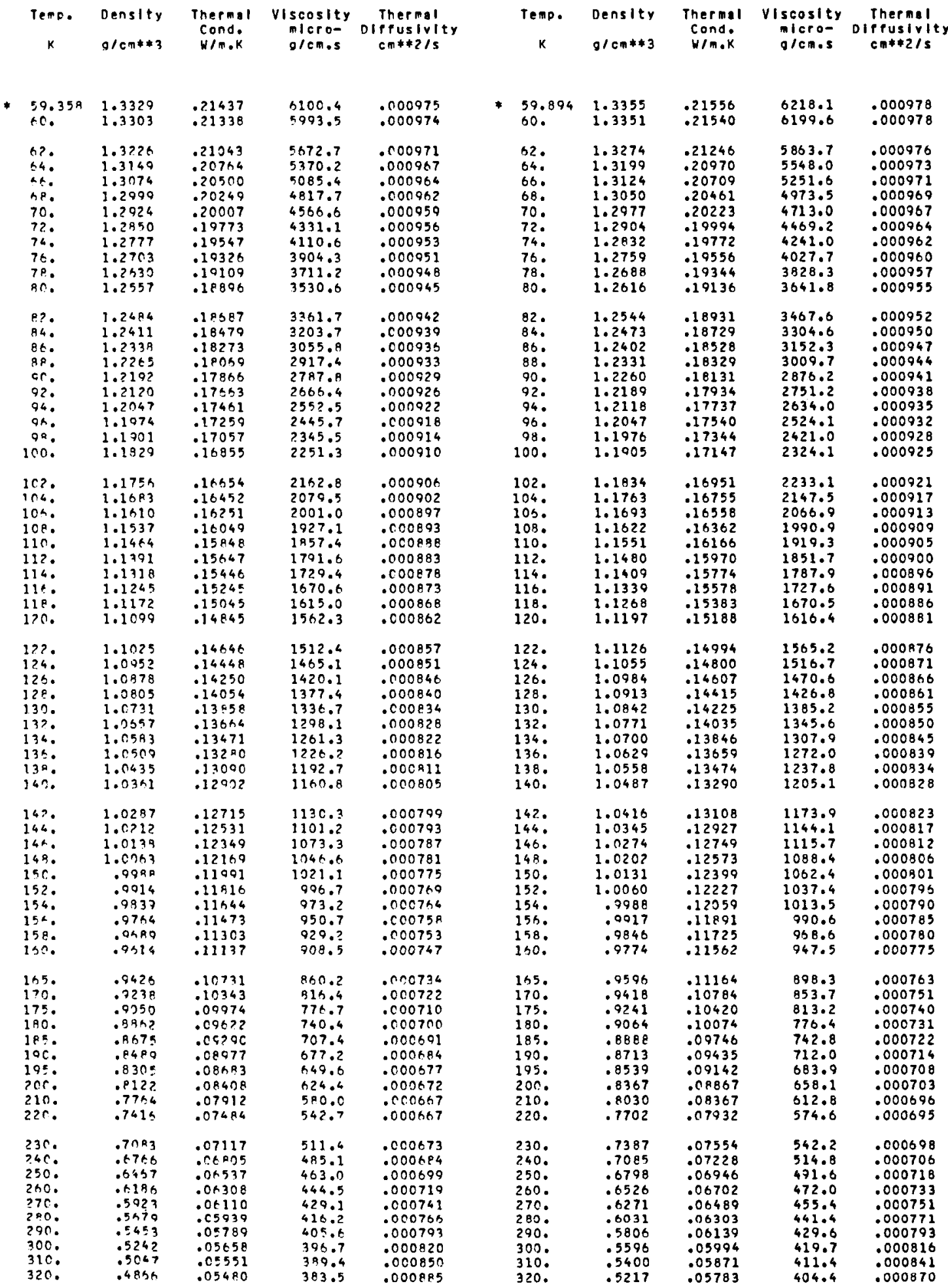

$50.0 \mathrm{MPE}$ Isober 
Table 5. Transport Propertles of axygen. Isobars, SI UnIts.

$55.0 \mathrm{MPa}$ Isobar

\begin{tabular}{|c|c|c|c|c|c|c|c|c|c|}
\hline $\begin{array}{c}\text { Teno. } \\
k\end{array}$ & $\begin{array}{l}\text { Density } \\
\text { g/en* } * 3\end{array}$ & $\begin{array}{c}\text { Thermal } \\
\text { Cond. } \\
\text { w/m.k }\end{array}$ & $\begin{array}{c}\text { VIscosity } \\
\text { rlero- } \\
\text { q/emos }\end{array}$ & $\begin{array}{c}\text { Thermal } \\
\text { olffusirity } \\
\text { cm*tzis }\end{array}$ & $\begin{array}{c}\text { Temp. } \\
k\end{array}$ & $\begin{array}{l}\text { Density } \\
g / c m * * 3\end{array}$ & $\begin{array}{l}\text { Thermal } \\
\text { Conds } \\
\text { H/mok }\end{array}$ & $\begin{array}{c}\text { VIscosity } \\
\text { micro- } \\
\text { g/cmos }\end{array}$ & $\begin{array}{c}\text { Thermal } \\
\text { olffusirity } \\
\text { cm*2 }\end{array}$ \\
\hline $\begin{array}{l}60.425 \\
62 . \\
64 . \\
60 . \\
68 . \\
70 . \\
7 ?: \\
74 . \\
76 . \\
78 . \\
80 .\end{array}$ & $\begin{array}{l}1.3381 \\
1.3321 \\
1.3246 \\
1.3173 \\
1.3100 \\
1.3028 \\
1.2958 \\
1.2985 \\
1.2814 \\
1.2743 \\
1.2673\end{array}$ & $\begin{array}{l}.21675 \\
.21446 \\
.21173 \\
.20915 \\
.20670 \\
.20436 \\
.20210 \\
.19992 \\
.19780 \\
.19573 \\
.19370\end{array}$ & $\begin{array}{l}6331.5 \\
6056.7 \\
5727.3 \\
5418.8 \\
5130.1 \\
4860.0 \\
4607.5 \\
4371.6 \\
4151.2 \\
3945.3 \\
3752.9\end{array}$ & $\begin{array}{l}.000981 \\
.000980 \\
.000979 \\
.000977 \\
.000975 \\
.000974 \\
.000972 \\
.000970 \\
.000968 \\
.000967 \\
.000965\end{array}$ & $\begin{array}{l}60.954 \\
62 . \\
64 . \\
66 . \\
68 . \\
70 . \\
72 . \\
74 . \\
76 . \\
78 . \\
80 .\end{array}$ & $\begin{array}{l}1.3407 \\
1.3367 \\
1.3293 \\
1.3220 \\
1.3148 \\
1.3077 \\
1.3006 \\
1.2936 \\
1.2867 \\
1.2797 \\
1.2728\end{array}$ & $\begin{array}{l}.21793 \\
.21643 \\
.21372 \\
.21116 \\
.20875 \\
.20644 \\
.20422 \\
.20208 \\
.20000 \\
.19798 \\
.19599\end{array}$ & $\begin{array}{l}6440.8 \\
6252.5 \\
5908.1 \\
5587.2 \\
5287.5 \\
5007.6 \\
4746.3 \\
4502.4 \\
4274.7 \\
4062.2 \\
3863.9\end{array}$ & $\begin{array}{l}.000984 \\
.000984 \\
.000984 \\
.000983 \\
.000982 \\
.000981 \\
.000979 \\
.000978 \\
.000977 \\
.000975 \\
.000974\end{array}$ \\
\hline $\begin{array}{l}82 . \\
84 . \\
86 . \\
88 . \\
90 . \\
92 . \\
94 . \\
96 . \\
98 . \\
100 .\end{array}$ & $\begin{array}{l}1.2603 \\
1.2533 \\
1.2463 \\
1.2394 \\
1.2324 \\
1.2255 \\
1.2195 \\
1.2116 \\
1.2047 \\
1.1978\end{array}$ & $\begin{array}{l}.19170 \\
.18973 \\
.18777 \\
.18583 \\
.18390 \\
.18197 \\
.18005 \\
.17914 \\
.176 ? 2 \\
.17431\end{array}$ & $\begin{array}{l}3573.2 \\
3405.3 \\
3248.3 \\
3101.5 \\
2964.1 \\
2835.4 \\
2714.0 \\
2601.8 \\
2495.8 \\
2396.2\end{array}$ & $\begin{array}{l}.000963 \\
.000960 \\
.000958 \\
.000956 \\
.000953 \\
.000950 \\
.000948 \\
.000945 \\
.000942 \\
.000938\end{array}$ & $\begin{array}{l}82 . \\
84 . \\
85 . \\
88 . \\
90 . \\
92 . \\
94 . \\
96 . \\
98 . \\
100 .\end{array}$ & $\begin{array}{l}1.2660 \\
1.2591 \\
1.2523 \\
1.2455 \\
1.2387 \\
1.2319 \\
1.2251 \\
1.2183 \\
1.2116 \\
1.2048\end{array}$ & $\begin{array}{l}.19404 \\
.19211 \\
.19020 \\
.18830 \\
.18642 \\
.18454 \\
.18267 \\
.18080 \\
.17894 \\
.17707\end{array}$ & $\begin{array}{l}3678.7 \\
3505.7 \\
3344.0 \\
3192.9 \\
3051.5 \\
2919.1 \\
2795.2 \\
2679.0 \\
2570.0 \\
2467.6\end{array}$ & $\begin{array}{l}.000972 \\
.000970 \\
.000968 \\
.000966 \\
.000964 \\
.000962 \\
.000960 \\
.000957 \\
.000954 \\
.000951\end{array}$ \\
\hline $\begin{array}{l}102 . \\
104 . \\
104 . \\
108 . \\
110 . \\
112 . \\
114 . \\
116 . \\
118 . \\
120 .\end{array}$ & $\begin{array}{l}1.1909 \\
1.1240 \\
1.1771 \\
1.1702 \\
1.1636 \\
1.1565 \\
1.1496 \\
1.1427 \\
1.1359 \\
1.1290\end{array}$ & $\begin{array}{l}.17240 \\
.17048 \\
.16857 \\
.16665 \\
.16474 \\
.16292 \\
.16091 \\
.15900 \\
.15709 \\
.15518\end{array}$ & $\begin{array}{l}2302.7 \\
2214.7 \\
2131.8 \\
2053.8 \\
1980.3 \\
1910.9 \\
1645.4 \\
1783.4 \\
1724.9 \\
1669.4\end{array}$ & $\begin{array}{l}.000935 \\
.000932 \\
.000928 \\
.000924 \\
.000920 \\
.000916 \\
.000912 \\
.000908 \\
.000903 \\
.000899\end{array}$ & $\begin{array}{l}102 . \\
104 . \\
106 . \\
108 . \\
110 . \\
112 . \\
114 . \\
116 . \\
118 . \\
120 .\end{array}$ & $\begin{array}{l}1.1981 \\
1.1914 \\
1.1847 \\
1.1779 \\
1.1712 \\
1.1645 \\
1.1579 \\
1.1512 \\
1.1445 \\
1.1378\end{array}$ & $\begin{array}{l}.17520 \\
.17333 \\
.17146 \\
.16959 \\
.16772 \\
.16585 \\
.16398 \\
.16211 \\
.16024 \\
.15838\end{array}$ & $\begin{array}{l}2371.5 \\
2281.1 \\
2196.0 \\
2115.9 \\
2040.4 \\
1969.2 \\
1901.9 \\
1838.4 \\
1778.4 \\
1721.4\end{array}$ & $\begin{array}{l}.000949 \\
.000945 \\
.000942 \\
.000939 \\
.000935 \\
.000931 \\
.000928 \\
.000924 \\
.000920 \\
.000916\end{array}$ \\
\hline $\begin{array}{l}122 . \\
124 . \\
126 . \\
128 . \\
130 . \\
1320 \\
134 . \\
1340 \\
138 . \\
140 .\end{array}$ & $\begin{array}{l}1.1221 \\
1.1153 \\
1.1294 \\
1.1016 \\
1.0947 \\
1.0879 \\
1.0810 \\
1.0741 \\
1.0673 \\
1.0504\end{array}$ & $\begin{array}{l}.15328 \\
.15179 \\
.14951 \\
.14763 \\
.14576 \\
.14391 \\
.14206 \\
.14023 \\
.13841 \\
.13561\end{array}$ & $\begin{array}{l}1516.9 \\
1567.1 \\
1519.8 \\
1475.0 \\
1432.3 \\
1301.8 \\
1353.2 \\
1316.4 \\
1281.4 \\
1247.9\end{array}$ & $\begin{array}{l}.000894 \\
.000890 \\
.000885 \\
.000880 \\
.000875 \\
.000870 \\
.000865 \\
.000850 \\
.000855 \\
.000850\end{array}$ & $\begin{array}{l}1220^{\circ} \\
124^{\circ} \\
126^{\circ} \\
128 . \\
130 . \\
1320^{\circ} \\
134^{\circ} \\
136^{\circ} \\
138^{\circ} \\
140^{\circ}\end{array}$ & $\begin{array}{l}1.1312 \\
1.1245 \\
1.1178 \\
1.1112 \\
1.1046 \\
1.0979 \\
1.0913 \\
1.0847 \\
1.0781 \\
1.0714\end{array}$ & $\begin{array}{l}.15652 \\
.15467 \\
.15282 \\
.15098 \\
.14915 \\
.14733 \\
.14552 \\
.14372 \\
.14194 \\
.14017\end{array}$ & $\begin{array}{l}1667.6 \\
1616.5 \\
1368.1 \\
1522.1 \\
1478.4 \\
1436.8 \\
1397.3 \\
1359.6 \\
1323.8 \\
1289.6\end{array}$ & $\begin{array}{l}.000911 \\
.000907 \\
.000903 \\
.000898 \\
.000894 \\
.000889 \\
.000884 \\
.000880 \\
.000875 \\
.000970\end{array}$ \\
\hline $\begin{array}{l}142 . \\
144 . \\
14 \% . \\
149 . \\
150 . \\
159 . \\
154 . \\
156 . \\
158 . \\
160 .\end{array}$ & $\begin{array}{r}1.0536 \\
1.0458 \\
1.0399 \\
1.0331 \\
1.0263 \\
1.0104 \\
1.0129 \\
1.0058 \\
.9990 \\
.9971\end{array}$ & $\begin{array}{l}.13482 \\
.13306 \\
.13131 \\
.12958 \\
.12787 \\
.12618 \\
.12453 \\
.12288 \\
.12125 \\
.11964\end{array}$ & $\begin{array}{r}1216.1 \\
1185.6 \\
1156.5 \\
1128.6 \\
1102.0 \\
1075.5 \\
1052.1 \\
1028.7 \\
1006.2 \\
084.7\end{array}$ & $\begin{array}{l}.000845 \\
.000840 \\
.000835 \\
.000830 \\
.000825 \\
.000820 \\
.000815 \\
.000810 \\
.000805 \\
.000800\end{array}$ & $\begin{array}{l}142 . \\
144 . \\
146 . \\
148 . \\
150 . \\
152 . \\
154 . \\
156 . \\
158 . \\
160 .\end{array}$ & $\begin{array}{l}1.0648 \\
1.0582 \\
1.0516 \\
1.0451 \\
1.0385 \\
1.0319 \\
1.0253 \\
1.0188 \\
1.0122 \\
1.0057\end{array}$ & $\begin{array}{l}.13842 \\
.13668 \\
.13496 \\
.13326 \\
.13158 \\
.12992 \\
.12829 \\
.12666 \\
.12506 \\
.12348\end{array}$ & $\begin{array}{l}1256.9 \\
1225.7 \\
1196.0 \\
1167.5 \\
1140.3 \\
1114.2 \\
1089.2 \\
1065.3 \\
1042.4 \\
1020.4\end{array}$ & $\begin{array}{l}.000866 \\
.000861 \\
.000856 \\
.000851 \\
.000847 \\
.000842 \\
.000837 \\
.000833 \\
.000828 \\
.000824\end{array}$ \\
\hline $\begin{array}{l}165 . \\
170 . \\
175 \\
170 \\
185 \\
190 \\
195 . \\
200 \\
210 . \\
220 .\end{array}$ & $\begin{array}{r}.0751 \\
.9582 \\
.9413 \\
.9245 \\
.9773 \\
.8912 \\
.8747 \\
.8594 \\
.8264 \\
.7953\end{array}$ & $\begin{array}{l}.11574 \\
.11199 \\
.10840 \\
.10498 \\
.10173 \\
.09855 \\
.09574 \\
.09299 \\
.08797 \\
.08355\end{array}$ & $\begin{array}{l}934.5 \\
889.0 \\
847.8 \\
910.2 \\
775.9 \\
744.6 \\
715.9 \\
689.8 \\
643.3 \\
604.0\end{array}$ & $\begin{array}{l}.000789 \\
.000778 \\
.000768 \\
.000758 \\
.000750 \\
.000743 \\
.000736 \\
.000731 \\
.000724 \\
.000721\end{array}$ & $\begin{array}{l}165 . \\
170 . \\
175 . \\
180 . \\
185 . \\
190 . \\
195 . \\
200 . \\
210 . \\
220 .\end{array}$ & $\begin{array}{r}.9994 \\
.9732 \\
.9570 \\
.9409 \\
.9250 \\
.9091 \\
.8935 \\
.8779 \\
.8474 \\
.8177\end{array}$ & $\begin{array}{l}.11963 \\
.11593 \\
.11239 \\
.10901 \\
.10578 \\
.10272 \\
.09982 \\
.09707 \\
.09203 \\
.08756\end{array}$ & $\begin{array}{l}969.1 \\
922.7 \\
880.6 \\
842.2 \\
807.3 \\
775.3 \\
746.0 \\
719.2 \\
671.8 \\
631.6\end{array}$ & $\begin{array}{l}.000813 \\
.000802 \\
.000793 \\
.000784 \\
.000776 \\
.000769 \\
.000762 \\
.000757 \\
.000749 \\
.000745\end{array}$ \\
\hline $\begin{array}{l}230 . \\
240 . \\
250 . \\
240 . \\
270 . \\
280 . \\
290 . \\
300 . \\
310 . \\
320 .\end{array}$ & $\begin{array}{l}.7653 \\
.7344 \\
.7048 \\
.4826 \\
.6578 \\
.6343 \\
.6122 \\
.5914 \\
.5719 \\
.5535\end{array}$ & $\begin{array}{l}.07968 \\
.07531 \\
.07336 \\
.07078 \\
.06852 \\
.06652 \\
.06475 \\
.06316 \\
.06180 \\
.06075\end{array}$ & $\begin{array}{l}570.7 \\
542.4 \\
518.2 \\
497.6 \\
480.1 \\
465.1 \\
452.3 \\
441.5 \\
432.2 \\
424.4\end{array}$ & $\begin{array}{l}.000722 \\
.000728 \\
.000736 \\
.000748 \\
.000763 \\
.000779 \\
.000798 \\
.000817 \\
.000838 \\
.000862\end{array}$ & $\begin{array}{l}230 . \\
240 . \\
250 . \\
260 . \\
270 . \\
280 . \\
290 . \\
300 . \\
310 . \\
320 .\end{array}$ & $\begin{array}{r}.7889 \\
.7613 \\
.7347 \\
.7093 \\
.6852 \\
.6623 \\
.6406 \\
.6201 \\
.6007 \\
.5824\end{array}$ & $\begin{array}{l}.08362 \\
.08015 \\
.07709 \\
.07439 \\
.07200 \\
.06987 \\
.06797 \\
.06627 \\
.06477 \\
.06356\end{array}$ & $\begin{array}{l}597.4 \\
568.2 \\
543.1 \\
521.7 \\
503.2 \\
487.4 \\
473.8 \\
462.2 \\
452.1 \\
443.6\end{array}$ & $\begin{array}{l}.000745 \\
.000749 \\
.000755 \\
.000764 \\
.000776 \\
.000790 \\
.000805 \\
.000821 \\
.000839 \\
.000859\end{array}$ \\
\hline
\end{tabular}

$60.0 \mathrm{MPa}$ Isobar 
Table 5. Transport propertles of Oxygen, Isobars, SI Units.

$65.0 \mathrm{MOa}$ I sobar

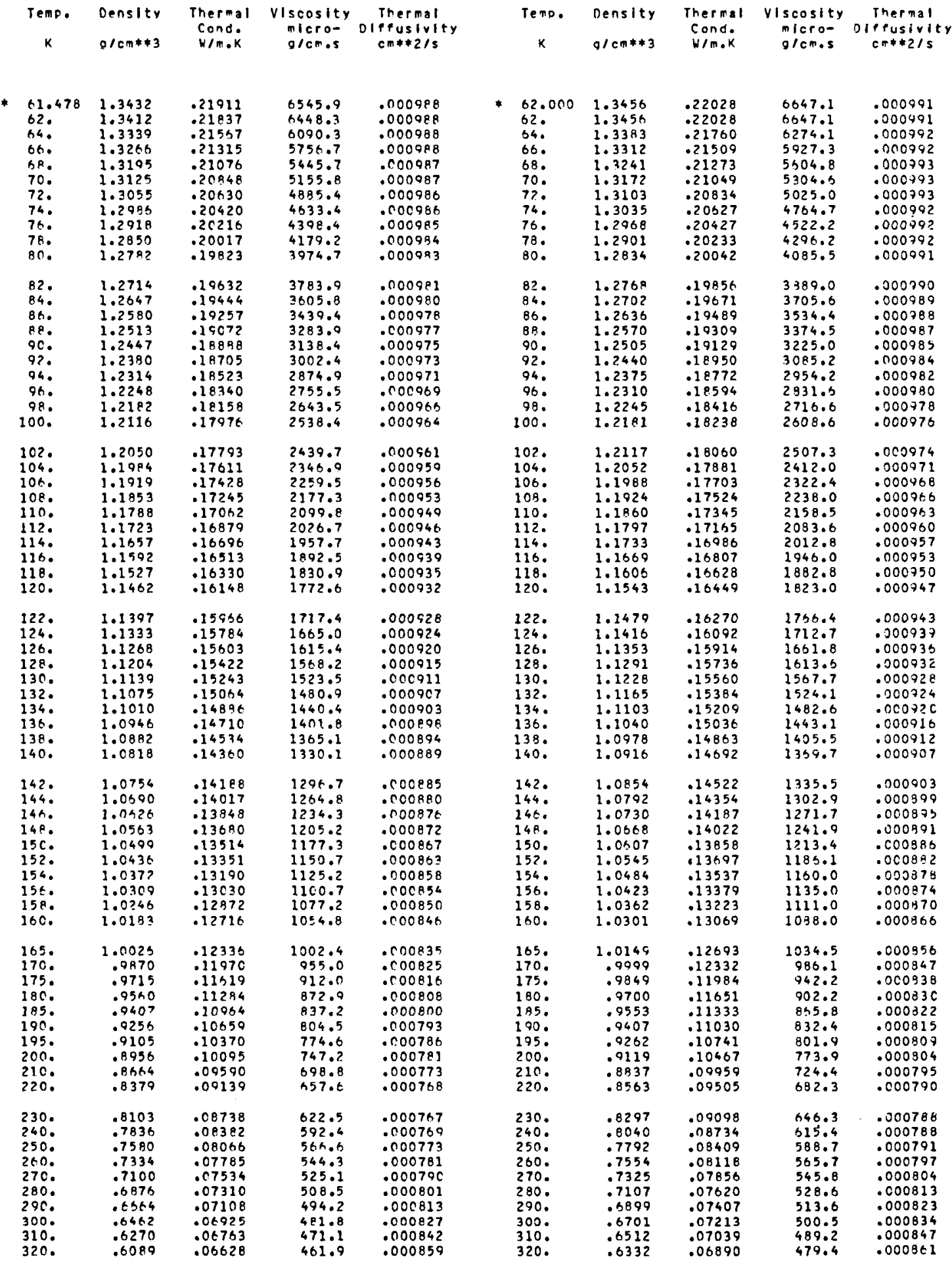


Table 5. Transport Propertles of Oxyoen, Isobars, SI Units.

$75.0 \mathrm{MP}$ Isober

\begin{tabular}{|c|c|c|c|c|c|c|c|c|c|}
\hline $\begin{array}{c}\text { Tenp. } \\
k\end{array}$ & $\begin{array}{l}\text { Density } \\
g / \mathrm{cm} * 3\end{array}$ & $\begin{array}{l}\text { Thermal } \\
\text { Cond. } \\
\text { W/m.k }\end{array}$ & $\begin{array}{c}\text { VIscosity } \\
m I c r o- \\
g / c m \text { s }\end{array}$ & $\begin{array}{c}\text { Thermal } \\
\text { Diffusivity } \\
\text { cm*\#2/s }\end{array}$ & $\begin{array}{c}\text { Tenp. } \\
k\end{array}$ & $\begin{array}{l}\text { Density } \\
\text { g/cm* } * 3\end{array}$ & $\begin{array}{c}\text { Thermas } \\
\text { Conde } \\
\text { w/mok }\end{array}$ & $\begin{array}{c}\text { VIscosity } \\
\text { micro- } \\
\text { g/cmos }\end{array}$ & $\begin{array}{c}\text { Thermal } \\
\text { olffusivit } \\
\text { cm*2/s }\end{array}$ \\
\hline $\begin{array}{l}62.518 \\
64 . \\
66 . \\
68 . \\
70 . \\
72 . \\
74 . \\
76 . \\
78 . \\
80 .\end{array}$ & $\begin{array}{l}1.3480 \\
1.3427 \\
1.3356 \\
1.3286 \\
1.3217 \\
1.3150 \\
1.3083 \\
1.3016 \\
1.2950 \\
1.2884\end{array}$ & $\begin{array}{l}.22146 \\
.21950 \\
.21701 \\
.21467 \\
.21246 \\
.21034 \\
.20831 \\
.20635 \\
.20444 \\
.20258\end{array}$ & $\begin{array}{l}6744.5 \\
6459.4 \\
6099.2 \\
5764.8 \\
5454.0 \\
5165.1 \\
4896.3 \\
4646.1 \\
4413.2 \\
4196.2\end{array}$ & $\begin{array}{l}.000994 \\
.000995 \\
.000997 \\
.000998 \\
.000998 \\
.000999 \\
.000999 \\
.000999 \\
.000999 \\
.000999\end{array}$ & $\begin{array}{l}63.032 \\
64 . \\
66 . \\
68 . \\
70 . \\
72 . \\
76 . \\
76 . \\
78 . \\
30 .\end{array}$ & $\begin{array}{l}1.3504 \\
1.3469 \\
1.3399 \\
1.3330 \\
1.3262 \\
1.3195 \\
1.3129 \\
1.3063 \\
1.2998 \\
1.2934\end{array}$ & $\begin{array}{l}.22263 \\
.22137 \\
.21890 \\
.21639 \\
.21440 \\
.21231 \\
.21031 \\
.20838 \\
.20651 \\
.20468\end{array}$ & $\begin{array}{l}6838.0 \\
6646.3 \\
6272.2 \\
5925.6 \\
5604.1 \\
5305.6 \\
5028.2 \\
4770.2 \\
4530.3 \\
4306.0\end{array}$ & $\begin{array}{l}.000997 \\
.000998 \\
.001000 \\
.001002 \\
.001004 \\
.001005 \\
.001005 \\
.001006 \\
.001006 \\
.001006\end{array}$ \\
\hline $\begin{array}{l}82 . \\
84 . \\
86 . \\
88 . \\
90 . \\
92 . \\
94 . \\
96 . \\
98 . \\
100 .\end{array}$ & $\begin{array}{l}1.2819 \\
1.2754 \\
1.2600 \\
1.2625 \\
1.2561 \\
1.2497 \\
1.2434 \\
1.2370 \\
1.2307 \\
1.2244\end{array}$ & $\begin{array}{l}.20075 \\
.19894 \\
.19716 \\
.19540 \\
.19365 \\
.19190 \\
.19016 \\
.18842 \\
.18668 \\
.18494\end{array}$ & $\begin{array}{l}3993.9 \\
3805.3 \\
3629.2 \\
3464.9 \\
3311.2 \\
3167.6 \\
3033.1 \\
2907.2 \\
2789.1 \\
2678.3\end{array}$ & $\begin{array}{l}.000998 \\
.000998 \\
.000997 \\
.000996 \\
.000995 \\
.000994 \\
.000992 \\
.000991 \\
.000989 \\
.000987\end{array}$ & $\begin{array}{l}82 . \\
84 . \\
86 . \\
88 . \\
90 . \\
92 . \\
94 . \\
96 . \\
98 . \\
100 .\end{array}$ & $\begin{array}{l}1.2869 \\
1.2806 \\
1.2742 \\
1.2679 \\
1.2616 \\
1.2553 \\
1.2491 \\
1.2428 \\
1.2366 \\
1.2304\end{array}$ & $\begin{array}{l}.20289 \\
.20113 \\
.19939 \\
.19767 \\
.19595 \\
.19425 \\
.19254 \\
.19084 \\
.18914 \\
.18744\end{array}$ & $\begin{array}{l}4098.8 \\
3904.8 \\
3723.8 \\
3554.9 \\
3397.1 \\
3249.6 \\
3111.6 \\
2982.3 \\
2861.2 \\
2747.6\end{array}$ & $\begin{array}{l}.001006 \\
.001006 \\
.001006 \\
.001005 \\
.001004 \\
.001004 \\
.001002 \\
.001001 \\
.001000 \\
.000998\end{array}$ \\
\hline $\begin{array}{l}102 . \\
104 . \\
106 . \\
108 . \\
110 . \\
112 . \\
114 . \\
116 . \\
118 . \\
120 .\end{array}$ & $\begin{array}{l}1.2181 \\
1.2118 \\
1.2055 \\
1.1992 \\
1.1930 \\
1.1868 \\
1.1805 \\
1.1743 \\
1.1681 \\
1.1620\end{array}$ & $\begin{array}{l}.18320 \\
.18145 \\
.17970 \\
.17795 \\
.17620 \\
.17445 \\
.17269 \\
.17093 \\
.16917 \\
.16742\end{array}$ & $\begin{array}{l}2574.3 \\
2476.6 \\
2384.7 \\
2298.2 \\
2216.6 \\
2139.8 \\
2047.2 \\
1998.7 \\
1933.9 \\
1872.7\end{array}$ & $\begin{array}{l}.000985 \\
.000983 \\
.000981 \\
.000978 \\
.000976 \\
.000973 \\
.000970 \\
.000967 \\
.000964 \\
.000961\end{array}$ & $\begin{array}{l}102 . \\
104 . \\
106 . \\
108 . \\
110 . \\
112 . \\
114 . \\
116 . \\
110 . \\
120 .\end{array}$ & $\begin{array}{l}1.2242 \\
1.2181 \\
1.2119 \\
1.2058 \\
1.1997 \\
1.1936 \\
1.1875 \\
1.1814 \\
1.1754 \\
1.1693\end{array}$ & $\begin{array}{r}.18574 \\
.18403 \\
.18232 \\
.18061 \\
.17889 \\
.17717 \\
.17545 \\
.17372 \\
.17200 \\
.17027\end{array}$ & $\begin{array}{l}2640.9 \\
2540.7 \\
2446.4 \\
2357.7 \\
2274.2 \\
2195.4 \\
2121.0 \\
2050.8 \\
1984.5 \\
1921.7\end{array}$ & $\begin{array}{l}.000997 \\
.000995 \\
.000993 \\
.000991 \\
.000988 \\
.000986 \\
.000983 \\
.000981 \\
.000978 \\
.000975\end{array}$ \\
\hline $\begin{array}{l}122 . \\
124 . \\
126 . \\
128 . \\
130 . \\
132 . \\
134 . \\
136 . \\
138 . \\
140 .\end{array}$ & $\begin{array}{l}1.1558 \\
1.1496 \\
1.1435 \\
1.1374 \\
1.1313 \\
1.1252 \\
1.1191 \\
1.1130 \\
1.1069 \\
1.1009\end{array}$ & $\begin{array}{l}.16566 \\
.16391 \\
.16216 \\
.16042 \\
.15868 \\
.15695 \\
.15523 \\
.15352 \\
.15182 \\
.15013\end{array}$ & $\begin{array}{l}1814.7 \\
1759.7 \\
1707.6 \\
1658.1 \\
1611.2 \\
1566.5 \\
1524.0 \\
1483.6 \\
1445.1 \\
1408.4\end{array}$ & $\begin{array}{l}.000958 \\
.000954 \\
.000951 \\
.000947 \\
.000944 \\
.000940 \\
.000936 \\
.000932 \\
.000928 \\
.000924\end{array}$ & $\begin{array}{l}122 . \\
124 . \\
126 . \\
128 . \\
130 . \\
132 . \\
134 . \\
135 . \\
138 . \\
140 .\end{array}$ & $\begin{array}{l}1.1633 \\
1.1573 \\
1.1513 \\
1.1453 \\
1.1394 \\
1.1334 \\
1.1275 \\
1.1216 \\
1.1157 \\
1.1098\end{array}$ & $\begin{array}{l}.16855 \\
.16683 \\
.16511 \\
.16339 \\
.16168 \\
.15998 \\
.15828 \\
.15660 \\
.15492 \\
.15326\end{array}$ & $\begin{array}{l}1862.3 \\
1806.0 \\
1752.7 \\
1702.0 \\
1653.9 \\
1608.2 \\
1564.8 \\
1523.4 \\
1484.0 \\
1444.5\end{array}$ & $\begin{array}{l}.000972 \\
.000969 \\
.000965 \\
.000962 \\
.000959 \\
.000955 \\
.000952 \\
.000948 \\
.000944 \\
.000941\end{array}$ \\
\hline $\begin{array}{l}142 . \\
144 . \\
146 . \\
148 . \\
150 . \\
152 . \\
154 . \\
156 . \\
150^{\circ} \\
150 .\end{array}$ & $\begin{array}{l}1.0949 \\
1.0889 \\
1.0828 \\
1.0768 \\
1.0709 \\
1.0649 \\
1.0590 \\
1.0530 \\
1.0471 \\
1.0412\end{array}$ & $\begin{array}{l}.14846 \\
.14680 \\
.14515 \\
.14352 \\
.14190 \\
.14031 \\
.13973 \\
.13717 \\
.13543 \\
.13410\end{array}$ & $\begin{array}{l}1373.5 \\
1340.1 \\
1308.2 \\
1277.7 \\
1248.5 \\
1220.7 \\
1194.0 \\
1168.4 \\
1143.9 \\
1120.4\end{array}$ & $\begin{array}{l}.000921 \\
.000917 \\
.000913 \\
.000900 \\
.000905 \\
.000901 \\
.000897 \\
.000893 \\
.000889 \\
.000985\end{array}$ & $\begin{array}{l}142 . \\
144 . \\
140^{\circ} \\
148^{\circ} \\
150^{\circ} \\
152 . \\
154 . \\
156 . \\
150^{\circ} \\
160 .\end{array}$ & $\begin{array}{l}1.1039 \\
1.0980 \\
1.0922 \\
1.0863 \\
1.0805 \\
1.0747 \\
1.0689 \\
1.0632 \\
1.0574 \\
1.0917\end{array}$ & $\begin{array}{l}.15160 \\
.14996 \\
.14833 \\
.14672 \\
.14513 \\
.14353 \\
.14199 \\
.14044 \\
.13891 \\
.13740\end{array}$ & $\begin{array}{l}1410.7 \\
1376.5 \\
1343.9 \\
1312.7 \\
1282.9 \\
1254.4 \\
1227.1 \\
1200.9 \\
1175.9 \\
1151.9\end{array}$ & $\begin{array}{l}.000937 \\
.000933 \\
.000931 \\
.000726 \\
.000922 \\
.000718 \\
.000913 \\
.000911 \\
.000907 \\
.000904\end{array}$ \\
\hline $\begin{array}{l}16 \% \\
170 . \\
175 . \\
180 . \\
185 . \\
190 . \\
195 . \\
200 . \\
210 . \\
220 .\end{array}$ & $\begin{array}{r}1.0245 \\
1.0119 \\
.9974 \\
.9431 \\
.9588 \\
.9547 \\
.9407 \\
.9269 \\
.8997 \\
.8733\end{array}$ & $\begin{array}{l}.13038 \\
.12680 \\
.12335 \\
.12004 \\
.11688 \\
.11385 \\
.11097 \\
.10823 \\
.10314 \\
.09856\end{array}$ & $\begin{array}{r}1065.7 \\
1016.2 \\
971.3 \\
930.5 \\
893.3 \\
859.2 \\
828.0 \\
799.4 \\
748.8 \\
705.8\end{array}$ & $\begin{array}{l}.000876 \\
.000867 \\
.000858 \\
.000850 \\
.000843 \\
.009836 \\
.000830 \\
.000825 \\
.000816 \\
.000810\end{array}$ & $\begin{array}{l}165 . \\
170 . \\
175 . \\
180 . \\
185 . \\
190 . \\
195 . \\
200 . \\
210 . \\
220 .\end{array}$ & $\begin{array}{r}1.0374 \\
1.0233 \\
1.0092 \\
.9953 \\
.9815 \\
.9678 \\
.9543 \\
.9409 \\
.9146 \\
.8890\end{array}$ & $\begin{array}{r}.13372 \\
.13016 \\
.12673 \\
.12344 \\
.12029 \\
.11728 \\
.11144 \\
.11166 \\
.10556 \\
.10194\end{array}$ & $\begin{array}{r}1096.0 \\
1045.4 \\
979.6 \\
957.9 \\
919.8 \\
885.1 \\
853.2 \\
824.0 \\
772.3 \\
728.3\end{array}$ & $\begin{array}{l}.000395 \\
.000886 \\
.000878 \\
.000870 \\
.000563 \\
.000856 \\
.000850 \\
.000945 \\
.000936 \\
.000830\end{array}$ \\
\hline $\begin{array}{l}230 . \\
240 . \\
250 . \\
260 . \\
270 . \\
280 . \\
290 . \\
300 . \\
310 . \\
320 .\end{array}$ & $\begin{array}{l}.8475 \\
.8226 \\
.7985 \\
.7755 \\
.7532 \\
.7319 \\
.7115 \\
.6921 \\
.6735 \\
.6557\end{array}$ & $\begin{array}{l}.09444 \\
.09073 \\
.08740 \\
.08439 \\
.08167 \\
.07921 \\
.07696 \\
.07491 \\
.07305 \\
.07143\end{array}$ & $\begin{array}{l}668.9 \\
637.2 \\
609.8 \\
536.1 \\
565.5 \\
547.6 \\
532.0 \\
518.4 \\
506.5 \\
496.1\end{array}$ & $\begin{array}{l}.000807 \\
.000807 \\
.000809 \\
.000812 \\
.000818 \\
.000824 \\
.000833 \\
.000842 \\
.000852 \\
.000864\end{array}$ & $\begin{array}{l}230 . \\
240 . \\
250 . \\
260 . \\
270 . \\
280 . \\
290 . \\
300 . \\
310 . \\
320 .\end{array}$ & $\begin{array}{l}.8641 \\
.8399 \\
.8165 \\
.7940 \\
.7724 \\
.7515 \\
.7316 \\
.7124 \\
.8941 \\
.6765\end{array}$ & $\begin{array}{l}.09777 \\
.09400 \\
.09059 \\
.08750 \\
.08468 \\
.09211 \\
.07975 \\
.07759 \\
.07562 \\
.07388\end{array}$ & $\begin{array}{l}590.5 \\
558.0 \\
630.0 \\
605.6 \\
534.4 \\
565.9 \\
549.7 \\
535.5 \\
523.1 \\
512.2\end{array}$ & $\begin{array}{l}.000826 \\
.000825 \\
.000325 \\
.000828 \\
.000932 \\
.000837 \\
.000844 \\
.000851 \\
.000859 \\
.000869\end{array}$ \\
\hline
\end{tabular}

80.0 MP. Isobar 
Tatle 5. Transoort Prodertles of Oxvaen. Isobars, Si Units.

65.C MOa Isotar

\begin{tabular}{|c|c|c|c|c|c|c|c|c|c|}
\hline $\begin{array}{c}\text { Teme. } \\
k\end{array}$ & $\begin{array}{l}\text { nensitv } \\
\text { a/en* } * 3\end{array}$ & $\begin{array}{l}\text { Thertal } \\
\text { cons. } \\
\text { w/m.k }\end{array}$ & $\begin{array}{c}\text { VIscosity } \\
\text { micro- } \\
0 / 0 m .5\end{array}$ & $\begin{array}{c}\text { Thermal } \\
\text { Diffusivitr } \\
\text { em*2is }\end{array}$ & $\begin{array}{c}\text { Tendo. } \\
k\end{array}$ & $\begin{array}{l}\text { Denslty } \\
\text { g/cm**3 }\end{array}$ & $\begin{array}{l}\text { Thernal } \\
\text { Cond. } \\
\text { W/mok }\end{array}$ & $\begin{array}{c}\text { Viscosity } \\
\text { micro- } \\
\text { g/cmos }\end{array}$ & $\begin{array}{c}\text { Thermal } \\
\text { Diffusivity } \\
\text { cm*2is }\end{array}$ \\
\hline $\begin{array}{l}63.564 \\
64 . \\
64 . \\
68 . \\
70 . \\
72 . \\
74 . \\
74: \\
78 . \\
80 .\end{array}$ & $\begin{array}{l}1.3527 \\
1.3511 \\
1.3441 \\
1.2373 \\
1.3305 \\
1.3730 \\
1.3174 \\
1.3109 \\
1.3065 \\
1.2781\end{array}$ & $\begin{array}{l}.22390 \\
.22321 \\
.22074 \\
.21947 \\
.21531 \\
.21425 \\
.21228 \\
.21039 \\
.20955 \\
.20474\end{array}$ & $\begin{array}{l}6927.0 \\
4834.9 \\
6446.5 \\
6087.5 \\
5755.0 \\
5446.5 \\
5150.4 \\
4994.5 \\
4647.5 \\
4417.5\end{array}$ & $\begin{array}{l}.001000 \\
.001001 \\
.001004 \\
.001003 \\
.001008 \\
.001010 \\
.001011 \\
.001012 \\
.001013 \\
.001014\end{array}$ & $\begin{array}{l}\text { * } 64.052 \\
56 . \\
58 . \\
70 . \\
7 ? . \\
74 . \\
76 . \\
78 . \\
80 .\end{array}$ & $\begin{array}{l}1.3550 \\
1.3483 \\
1.3415 \\
1.3348 \\
1.3283 \\
1.3218 \\
1.3154 \\
1.3091 \\
1.3028\end{array}$ & $\begin{array}{l}.22497 \\
.22260 \\
.22032 \\
.21818 \\
.21615 \\
.21422 \\
.21235 \\
.21055 \\
.20879\end{array}$ & $\begin{array}{l}7014.2 \\
6622.2 \\
6250.3 \\
5906.5 \\
5588.2 \\
5293.1 \\
5019.2 \\
4764.8 \\
4528.3\end{array}$ & $\begin{array}{l}.001003 \\
.001007 \\
.001010 \\
.001013 \\
.001015 \\
.001017 \\
.001018 \\
.001020 \\
.001021\end{array}$ \\
\hline $\begin{array}{l}82 . \\
84 . \\
8 \%: \\
92 . \\
90 . \\
9 ? . \\
96 . \\
98 . \\
98 . \\
100 .\end{array}$ & $\begin{array}{l}1.2918 \\
1.2855 \\
1.2703 \\
1.2731 \\
1.2469 \\
1.2407 \\
1.2546 \\
1.2485 \\
1.2474 \\
1.2363\end{array}$ & $\begin{array}{l}.20500 \\
.20328 \\
.20157 \\
.19929 \\
.19821 \\
.19654 \\
.19488 \\
.19322 \\
.19156 \\
.12909\end{array}$ & $\begin{array}{l}4203.5 \\
4004.1 \\
3918.2 \\
3644.9 \\
3482.8 \\
3331.3 \\
3189.7 \\
3057.1 \\
2932.9 \\
2816.4\end{array}$ & $\begin{array}{l}.001014 \\
.001014 \\
.001014 \\
.001014 \\
.001013 \\
.001013 \\
.001012 \\
.001011 \\
.001010 \\
.001009\end{array}$ & $\begin{array}{l}82 . \\
84 . \\
85 . \\
88 . \\
90 . \\
92 . \\
94 . \\
95 . \\
98 . \\
100 .\end{array}$ & $\begin{array}{l}1.2966 \\
1.2904 \\
1.2842 \\
1.2781 \\
1.2720 \\
1.2650 \\
1.2599 \\
1.2539 \\
1.2470 \\
1.2420\end{array}$ & $\begin{array}{l}.20707 \\
.20538 \\
.20372 \\
.20207 \\
.20043 \\
.19880 \\
.19717 \\
.19555 \\
.19392 \\
.19229\end{array}$ & $\begin{array}{l}4308.3 \\
4103.4 \\
3912.5 \\
3734.4 \\
3568.1 \\
3412.8 \\
3267.5 \\
3131.6 \\
3004.2 \\
2884.8\end{array}$ & $\begin{array}{l}.001021 \\
.001022 \\
.001022 \\
.001022 \\
.001022 \\
.001022 \\
.001021 \\
.001021 \\
.001020 \\
.001019\end{array}$ \\
\hline $\begin{array}{l}107 . \\
104 . \\
106 . \\
108 . \\
118 . \\
112 . \\
114 . \\
116 . \\
118 . \\
1 \geqslant 0 .\end{array}$ & $\begin{array}{l}1.2302 \\
1.224 ? \\
1.2182 \\
1.21 ? 2 \\
1.2052 \\
1.2002 \\
1.1942 \\
1.1283 \\
1.1824 \\
1.1765\end{array}$ & $\begin{array}{l}.18923 \\
.18656 \\
.18498 \\
.18320 \\
.18152 \\
.17983 \\
.17814 \\
.17645 \\
.17475 \\
.17306\end{array}$ & $\begin{array}{l}2707.0 \\
2504.3 \\
2507.7 \\
2416.8 \\
2331.2 \\
2250.5 \\
2174.3 \\
2102.4 \\
2034.5 \\
1070.2\end{array}$ & $\begin{array}{l}.001007 \\
.001006 \\
.001004 \\
.001002 \\
.001000 \\
.000998 \\
.000996 \\
.000993 \\
.000991 \\
.000984\end{array}$ & $\begin{array}{l}102 . \\
104 . \\
105 . \\
109 . \\
110 . \\
112 . \\
114 . \\
116 . \\
118 . \\
120 .\end{array}$ & $\begin{array}{l}1.2360 \\
1.2301 \\
1.2242 \\
1.2183 \\
1.2124 \\
1.2066 \\
1.2007 \\
1.1949 \\
1.1891 \\
1.1833\end{array}$ & $\begin{array}{l}.19066 \\
.18903 \\
.18739 \\
.18574 \\
.18409 \\
.18243 \\
.18078 \\
.17911 \\
.17745 \\
.17578\end{array}$ & $\begin{array}{l}2772.7 \\
2667.5 \\
2568.5 \\
2475.4 \\
2387.7 \\
2305.1 \\
2227.1 \\
2153.5 \\
2083.9 \\
2018.2\end{array}$ & $\begin{array}{l}.001018 \\
.001017 \\
.001015 \\
.0010114 \\
.001012 \\
.001010 \\
.001008 \\
.001006 \\
.001003 \\
.001001\end{array}$ \\
\hline $\begin{array}{l}122 . \\
124 . \\
126 . \\
128 . \\
130 . \\
132 . \\
134 . \\
136 . \\
138 . \\
140 .\end{array}$ & $\begin{array}{l}1.1704 \\
1.1547 \\
1.1588 \\
1.1530 \\
1.1471 \\
1.1413 \\
1.1355 \\
1.1297 \\
1.1240 \\
1.1182\end{array}$ & $\begin{array}{l}.17139 \\
.16967 \\
.16798 \\
.16679 \\
.16451 \\
.16293 \\
.16126 \\
.15959 \\
.15794 \\
.15629\end{array}$ & $\begin{array}{l}1909.4 \\
1851.8 \\
1797.1 \\
1745.3 \\
1696.1 \\
1649.3 \\
1604.8 \\
1542.5 \\
1522.7 \\
1463.8\end{array}$ & $\begin{array}{l}.000985 \\
.000982 \\
.000979 \\
.000974 \\
.000973 \\
.000970 \\
.000967 \\
.000943 \\
.000960 \\
.000956\end{array}$ & $\begin{array}{l}122 . \\
124 . \\
126 . \\
128^{\circ} \\
130 . \\
132 . \\
134 . \\
136 . \\
138^{\circ} \\
140 .\end{array}$ & $\begin{array}{l}1.1775 \\
1.1718 \\
1.1660 \\
1.1603 \\
1.1546 \\
1.1489 \\
1.1433 \\
1.1376 \\
1.1320 \\
1.1263\end{array}$ & $\begin{array}{l}.17411 \\
.17245 \\
.17078 \\
.16912 \\
.16746 \\
.16580 \\
.16416 \\
.16251 \\
.16088 \\
.15926\end{array}$ & $\begin{array}{l}1955.9 \\
1896.9 \\
1841.0 \\
1780.0 \\
1737.6 \\
1689.8 \\
1644.3 \\
1601.0 \\
1559.8 \\
1520.5\end{array}$ & $\begin{array}{l}.000998 \\
.000996 \\
.000993 \\
.000990 \\
.000987 \\
.000984 \\
.000981 \\
.000978 \\
.000974 \\
.000971\end{array}$ \\
\hline $\begin{array}{l}142 . \\
144 . \\
140^{\circ} \\
148 . \\
150 . \\
1520^{\circ} \\
1540^{\circ} \\
150^{\circ} \\
158^{\circ} \\
160 .\end{array}$ & $\begin{array}{l}1.1125 \\
1.1068 \\
1.1911 \\
1.0954 \\
1.0897 \\
1.0841 \\
1.0784 \\
1.0728 \\
1.0572 \\
1.0616\end{array}$ & $\begin{array}{l}.15466 \\
.15304 \\
.15143 \\
.14944 \\
.14826 \\
.14669 \\
.14515 \\
.14362 \\
.14210 \\
.14061\end{array}$ & $\begin{array}{l}1447.2 \\
1412.2 \\
1378.9 \\
1347.0 \\
1316.5 \\
1287.4 \\
1259.5 \\
1232.7 \\
1207.1 \\
1182.6\end{array}$ & $\begin{array}{l}.000953 \\
.000949 \\
.000946 \\
.000942 \\
.000939 \\
.000935 \\
.000931 \\
.000928 \\
.000924 \\
.000921\end{array}$ & $\begin{array}{l}142 . \\
144 . \\
146 . \\
148 . \\
150 . \\
150^{\circ} \\
154^{\circ} \\
156^{\circ} \\
158^{\circ} \\
160 .\end{array}$ & $\begin{array}{l}1.1207 \\
1.1152 \\
1.1096 \\
1.1040 \\
1.0985 \\
1.0930 \\
1.0875 \\
1.0820 \\
1.0766 \\
1.0711\end{array}$ & $\begin{array}{l}.15764 \\
.15604 \\
.15445 \\
.15287 \\
.15131 \\
.14976 \\
.14822 \\
.14671 \\
.14520 \\
.14372\end{array}$ & $\begin{array}{l}1483.1 \\
1447.4 \\
1413.2 \\
1380.7 \\
1349.5 \\
1319.7 \\
1291.2 \\
1263.9 \\
1237.7 \\
1212.6\end{array}$ & $\begin{array}{l}.000968 \\
.000964 \\
.000961 \\
.000958 \\
.000954 \\
.000951 \\
.000947 \\
.000944 \\
.000941 \\
.000937\end{array}$ \\
\hline $\begin{array}{l}165 . \\
170 . \\
175 . \\
180 \\
185 \\
190 . \\
195 . \\
200 . \\
210 . \\
220 .\end{array}$ & $\begin{array}{r}1.0478 \\
1.0340 \\
1.0203 \\
1.0068 \\
.9934 \\
.9802 \\
.9670 \\
.9540 \\
.9285 \\
.9036\end{array}$ & $\begin{array}{l}.13695 \\
.13341 \\
.13001 \\
.12674 \\
.12360 \\
.12059 \\
.11772 \\
.11497 \\
.10985 \\
.10521\end{array}$ & $\begin{array}{r}1125.5 \\
1073.8 \\
1027.0 \\
984.4 \\
945.6 \\
910.1 \\
877.5 \\
847.7 \\
794.9 \\
749.9\end{array}$ & $\begin{array}{l}.000912 \\
.000904 \\
.000896 \\
.000888 \\
.000881 \\
.000875 \\
.000869 \\
.000864 \\
.000855 \\
.000848\end{array}$ & $\begin{array}{l}165 . \\
170 . \\
175 . \\
180 . \\
185 . \\
190 . \\
195 . \\
200 . \\
210 . \\
220 .\end{array}$ & $\begin{array}{r}1.0576 \\
1.0442 \\
1.0309 \\
1.0177 \\
1.0047 \\
.9918 \\
.9790 \\
.9664 \\
.9416 \\
.9174\end{array}$ & $\begin{array}{l}.14009 \\
.13658 \\
.13319 \\
.12993 \\
.12680 \\
.12380 \\
.12093 \\
.11818 \\
.111305 \\
.10837\end{array}$ & $\begin{array}{r}1154.3 \\
1101.5 \\
1053.7 \\
1010.2 \\
970.6 \\
934.3 \\
901.1 \\
870.6 \\
816.7 \\
770.7\end{array}$ & $\begin{array}{l}.000929 \\
.000921 \\
.000913 \\
.000906 \\
.000899 \\
.000893 \\
.000887 \\
.000882 \\
.000873 \\
.000866\end{array}$ \\
\hline $\begin{array}{l}230 . \\
240 . \\
250 . \\
260 . \\
270 \\
280 \\
290 \\
300 \\
310 . \\
320 .\end{array}$ & $\begin{array}{l}.8794 \\
.8560 \\
.8332 \\
.8113 \\
.7901 \\
.7698 \\
.7502 \\
.7314 \\
.7134 \\
.6961\end{array}$ & $\begin{array}{l}.10099 \\
.09716 \\
.09368 \\
.09050 \\
.08759 \\
.08492 \\
.08246 \\
.08020 \\
.07812 \\
.07625\end{array}$ & $\begin{array}{l}711.3 \\
678.0 \\
649.2 \\
624.2 \\
602.4 \\
583.3 \\
566.6 \\
551.9 \\
539.0 \\
527.0\end{array}$ & $\begin{array}{l}.000844 \\
.000842 \\
.000841 \\
.000843 \\
.000845 \\
.000849 \\
.000854 \\
.000860 \\
.000866 \\
.000874\end{array}$ & $\begin{array}{l}230 . \\
260 . \\
250 . \\
260 . \\
270 . \\
280 . \\
290 . \\
300 . \\
310 . \\
320 .\end{array}$ & $\begin{array}{r}.8938 \\
.8710 \\
.8488 \\
.8274 \\
.8067 \\
.7868 \\
.7676 \\
.7492 \\
.7314 \\
.7144\end{array}$ & $\begin{array}{l}.10411 \\
.10022 \\
.09667 \\
.09341 \\
.09041 \\
.08765 \\
.08509 \\
.08272 \\
.08054 \\
.07855\end{array}$ & $\begin{array}{l}731.3 \\
697.3 \\
667.8 \\
642.1 \\
619.7 \\
600.1 \\
582.8 \\
567.6 \\
554.3 \\
542.5\end{array}$ & $\begin{array}{l}.000861 \\
.000858 \\
.000857 \\
.000857 \\
.000859 \\
.000861 \\
.000865 \\
.000869 \\
.000873 \\
.000879\end{array}$ \\
\hline
\end{tabular}


Table 5. Transport Droperties of Cxygan, Isobars, si Units.

$95.0 \mathrm{MPo}$ Isooar

100.0 MPa Isobar

\begin{tabular}{|c|c|c|c|c|c|c|c|c|c|}
\hline $\begin{array}{c}\text { TEND. } \\
K\end{array}$ & $\begin{array}{l}\text { Density } \\
\text { a/cn**3 }\end{array}$ & $\begin{array}{l}\text { Thermal } \\
\text { Cond. } \\
\text { W/mok }\end{array}$ & $\begin{array}{c}\text { viscosity } \\
\text { micro- } \\
\text { g/emos }\end{array}$ & $\begin{array}{c}\text { Thermal } \\
\text { Diffuslvity } \\
\text { cm* } * 2 / s\end{array}$ & $\begin{array}{c}\text { Temp. } \\
k\end{array}$ & $\begin{array}{l}\text { Density } \\
\text { g/C**3 }\end{array}$ & $\begin{array}{l}\text { Thermal } \\
\text { Cond. } \\
\text { v/m.k }\end{array}$ & $\begin{array}{c}\text { VIscosity } \\
\text { mIcro- } \\
\text { g/emos }\end{array}$ & $\begin{array}{c}\text { Thermal } \\
\text { Diffusivity } \\
\text { cm**2/s }\end{array}$ \\
\hline $\begin{array}{l}82 . \\
84 . \\
85 . \\
88 . \\
90 . \\
92 . \\
94 . \\
96 . \\
98 . \\
100 .\end{array}$ & $\begin{array}{l}1.3012 \\
1.2951 \\
1.2890 \\
1.2830 \\
1.2770 \\
1.2711 \\
1.2651 \\
1.2592 \\
1.2533 \\
1.2475\end{array}$ & $\begin{array}{l}.20911 \\
.20745 \\
.20582 \\
.20421 \\
.20260 \\
.20101 \\
.19942 \\
.19783 \\
.19624 \\
.19465\end{array}$ & $\begin{array}{l}4413.0 \\
4202.6 \\
4006.5 \\
3823.8 \\
3453.2 \\
3493.9 \\
3345.0 \\
3205.7 \\
3075.2 \\
2952.8\end{array}$ & $\begin{array}{l}.001028 \\
.001029 \\
.001030 \\
.001030 \\
.001031 \\
.001031 \\
.001030 \\
.001030 \\
.001030 \\
.001029\end{array}$ & $\begin{array}{l}82 . \\
84 . \\
86 . \\
88 . \\
90 . \\
92 . \\
94 . \\
96 . \\
98 . \\
100 .\end{array}$ & $\begin{array}{l}1.3057 \\
1.2997 \\
1.2937 \\
1.2878 \\
1.2819 \\
1.2760 \\
1.2702 \\
1.2644 \\
1.2586 \\
1.2528\end{array}$ & $\begin{array}{l}.21111 \\
.20948 \\
.20789 \\
.20631 \\
.20474 \\
.20318 \\
.20163 \\
.20007 \\
.19851 \\
.19695\end{array}$ & $\begin{array}{l}4517.7 \\
4301.7 \\
4100.5 \\
3913.0 \\
3738.2 \\
3574.9 \\
3422.2 \\
3279.5 \\
3145.8 \\
3020.5\end{array}$ & $\begin{array}{l}.001035 \\
.001036 \\
.001037 \\
.001038 \\
.001039 \\
.001039 \\
.001039 \\
.001039 \\
.001039 \\
.001038\end{array}$ \\
\hline $\begin{array}{l}102 . \\
104 . \\
106 . \\
108 . \\
110 . \\
112 . \\
114 . \\
116 . \\
118 . \\
120 .\end{array}$ & $\begin{array}{l}1.2416 \\
1.2358 \\
1.2300 \\
1.2242 \\
1.2185 \\
1.2127 \\
1.2070 \\
1.2013 \\
1.1956 \\
1.1809\end{array}$ & $\begin{array}{l}.19305 \\
.19145 \\
.18984 \\
.18823 \\
.18861 \\
.18498 \\
.18336 \\
.18172 \\
.18009 \\
.17845\end{array}$ & $\begin{array}{l}2838.0 \\
2730.2 \\
2528.9 \\
2533.6 \\
2443.8 \\
2359.2 \\
2279.4 \\
2204.1 \\
2132.9 \\
2065.6\end{array}$ & $\begin{array}{l}.001028 \\
.001027 \\
.001626 \\
.001024 \\
.001023 \\
.001021 \\
.001019 \\
.001017 \\
.001015 \\
.001013\end{array}$ & $\begin{array}{l}102 . \\
104 . \\
105 . \\
108 . \\
110 . \\
112 . \\
114 . \\
116 . \\
118 . \\
120 .\end{array}$ & $\begin{array}{l}1.2471 \\
1.2414 \\
1.2357 \\
1.2300 \\
1.2243 \\
1.2187 \\
1.2131 \\
1.2074 \\
1.2019 \\
1.1963\end{array}$ & $\begin{array}{l}.19539 \\
.19382 \\
.19225 \\
.19066 \\
.18908 \\
.18748 \\
.18588 \\
.18428 \\
.18267 \\
.18106\end{array}$ & $\begin{array}{l}2903.0 \\
2792.6 \\
2688.9 \\
2591.3 \\
2499.5 \\
2412.9 \\
2331.3 \\
2254.3 \\
2181.5 \\
2112.6\end{array}$ & $\begin{array}{l}.001038 \\
.001037 \\
.001036 \\
.001035 \\
.001034 \\
.001032 \\
.001031 \\
.001029 \\
.001027 \\
.001025\end{array}$ \\
\hline $\begin{array}{l}122 . \\
124 . \\
126 . \\
128 . \\
130 . \\
132 . \\
134 . \\
136 . \\
130^{\circ} \\
140 .\end{array}$ & $\begin{array}{l}1.1843 \\
1.1786 \\
1.1730 \\
1.1674 \\
1.1618 \\
1.1542 \\
1.1507 \\
1.11451 \\
1.1396 \\
1.1341\end{array}$ & $\begin{array}{l}.17581 \\
.17517 \\
.17352 \\
.17189 \\
.17025 \\
.16852 \\
.16699 \\
.16537 \\
.16375 \\
.16215\end{array}$ & $\begin{array}{l}2001.9 \\
1941.6 \\
1884.4 \\
1830.2 \\
1778.7 \\
1729.8 \\
1633.2 \\
1639.0 \\
1596.8 \\
1556.7\end{array}$ & $\begin{array}{l}.001011 \\
.001008 \\
.001008 \\
.001003 \\
.001000 \\
.000997 \\
.000995 \\
.000992 \\
.000988 \\
.000985\end{array}$ & $\begin{array}{l}122 . \\
124 . \\
126 . \\
120^{\circ} \\
130 . \\
132 . \\
134 . \\
136^{\circ} \\
138^{\circ} \\
140 .\end{array}$ & $\begin{array}{l}1.1907 \\
1.1852 \\
1.1797 \\
1.1742 \\
1.1687 \\
1.1633 \\
1.1578 \\
1.1524 \\
1.1470 \\
1.11416\end{array}$ & $\begin{array}{l}.17944 \\
.17783 \\
.17621 \\
.17459 \\
.17298 \\
.17137 \\
.16976 \\
.16816 \\
.16656 \\
.16497\end{array}$ & $\begin{array}{l}2047.5 \\
1985.8 \\
1927.3 \\
1871.9 \\
1819.2 \\
1769.2 \\
1721.7 \\
1676.4 \\
1633.4 \\
1592.4\end{array}$ & $\begin{array}{l}.001023 \\
.001021 \\
.001018 \\
.001016 \\
.001013 \\
.001010 \\
.001008 \\
.001005 \\
.001002 \\
.000999\end{array}$ \\
\hline $\begin{array}{l}165 . \\
170 . \\
175 . \\
180 . \\
185 . \\
190 . \\
105 . \\
200 . \\
210 . \\
220 .\end{array}$ & $\begin{array}{r}1.0670 \\
1.0539 \\
1.0409 \\
1.0281 \\
1.0154 \\
1.0028 \\
.9003 \\
.9780 \\
.9530 \\
.9303\end{array}$ & $\begin{array}{l}.14315 \\
.13966 \\
.13628 \\
.13304 \\
.12991 \\
.12692 \\
.12404 \\
.12120 \\
.11614 \\
.11144\end{array}$ & $\begin{array}{r}1182.5 \\
1128.6 \\
1079.8 \\
1035.4 \\
994.9 \\
957.9 \\
92.4 .0 \\
892.9 \\
837.8 \\
790.9\end{array}$ & $\begin{array}{l}.000945 \\
.000937 \\
.000930 \\
.000923 \\
.000916 \\
.000910 \\
.000904 \\
.000899 \\
.000890 \\
.000883\end{array}$ & $\begin{array}{l}265 . \\
170 . \\
175 . \\
180 . \\
185 . \\
190 . \\
195 . \\
200 . \\
210 . \\
220 .\end{array}$ & $\begin{array}{r}1.0759 \\
1.0531 \\
1.0505 \\
1.0379 \\
1.0255 \\
1.0132 \\
1.0011 \\
.9891 \\
.9855 \\
.9425\end{array}$ & $\begin{array}{l}.14613 \\
.14265 \\
.13930 \\
.13606 \\
.13294 \\
.12995 \\
.12708 \\
.12432 \\
.11916 \\
.11442\end{array}$ & $\begin{array}{r}1210.1 \\
1155.1 \\
1105.3 \\
1059.9 \\
1018.6 \\
980.8 \\
946.2 \\
914.5 \\
858.3 \\
810.4\end{array}$ & $\begin{array}{l}.000961 \\
.000953 \\
.000946 \\
.000939 \\
.000932 \\
.000926 \\
.000921 \\
.000915 \\
.000906 \\
.000899\end{array}$ \\
\hline $\begin{array}{l}230 . \\
240 . \\
250 . \\
260 . \\
270 . \\
280 . \\
290 . \\
300 . \\
310 . \\
320 .\end{array}$ & $\begin{array}{l}.0073 \\
.8851 \\
.8634 \\
.8425 \\
.8223 \\
.8028 \\
.7839 \\
.7658 \\
.7484 \\
.7316\end{array}$ & $\begin{array}{l}.10713 \\
.10319 \\
.09957 \\
.09624 \\
.09316 \\
.09030 \\
.08765 \\
.08518 \\
.08289 \\
.08079\end{array}$ & $\begin{array}{l}750.6 \\
715.8 \\
685.6 \\
659.3 \\
636.4 \\
616.2 \\
508.5 \\
582.8 \\
569.0 \\
556.8\end{array}$ & $\begin{array}{l}.000877 \\
.000874 \\
.000872 \\
.000871 \\
.000872 \\
.000873 \\
.000875 \\
.000878 \\
.000881 \\
.000885\end{array}$ & $\begin{array}{l}230 . \\
240 . \\
250 . \\
260 . \\
270 . \\
280 . \\
290 . \\
300 . \\
310 . \\
320 .\end{array}$ & $\begin{array}{l}.0201 \\
.8983 \\
.8772 \\
.8568 \\
.8370 \\
.8178 \\
.7993 \\
.7815 \\
.7643 \\
.7478\end{array}$ & $\begin{array}{l}.11008 \\
.10608 \\
.10240 \\
.09899 \\
.09583 \\
.09289 \\
.09024 \\
.08757 \\
.08517 \\
.08296\end{array}$ & $\begin{array}{l}769.3 \\
733.7 \\
702.9 \\
676.0 \\
652.4 \\
631.8 \\
613.5 \\
597.5 \\
583.2 \\
570.6\end{array}$ & $\begin{array}{l}.000893 \\
.000889 \\
.000886 \\
.000885 \\
.000884 \\
.000884 \\
.000885 \\
.000887 \\
.000888 \\
.000891\end{array}$ \\
\hline
\end{tabular}

- Two phase Boundary 


\section{This Page Intentionally Left Blank}


Table 6. Transoort procepties of Dxygen for saturatac lizuid and vapop, fagr. Units

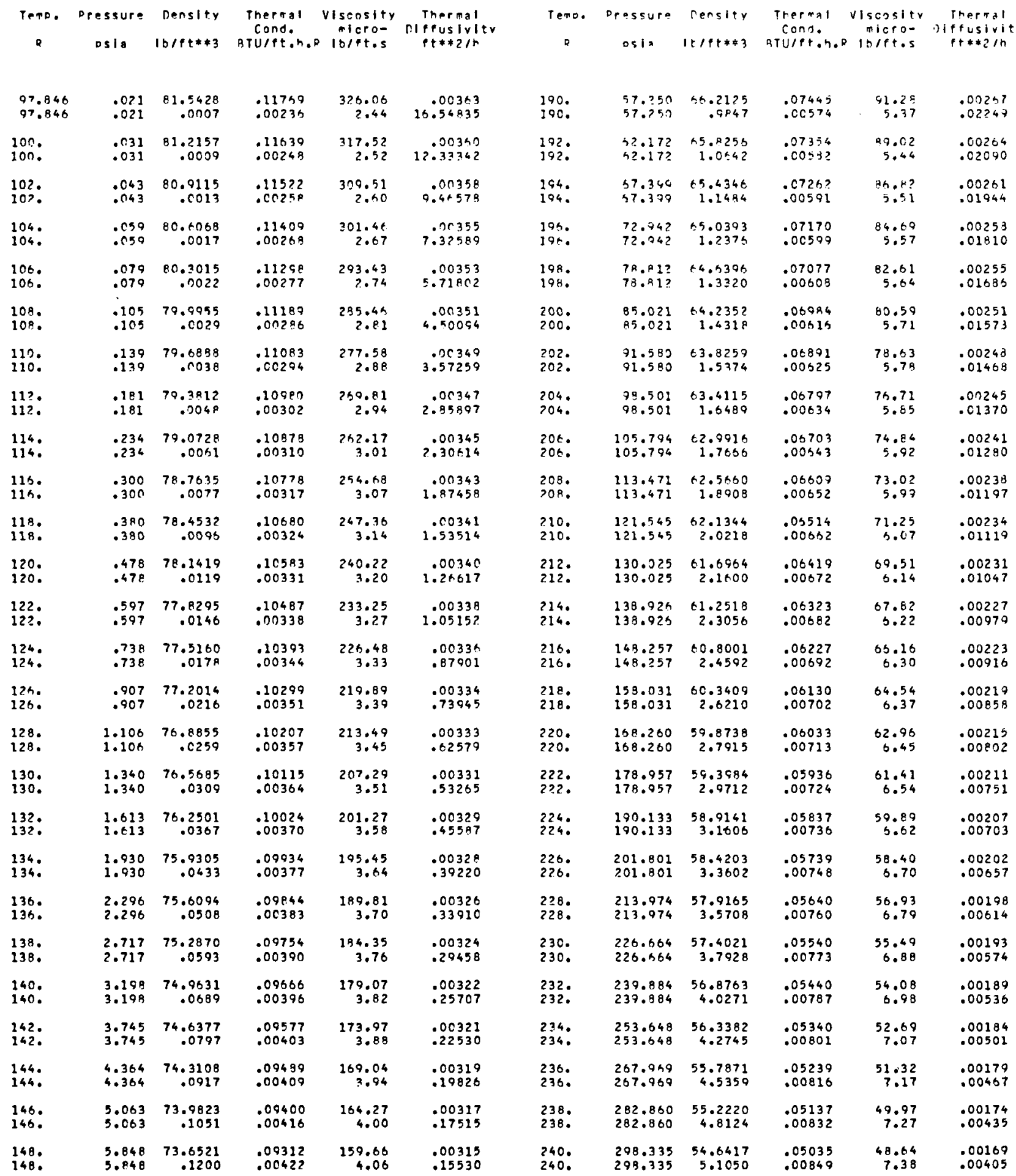


Table 6. Transport Propertles of oxyoen for saturoted Llauld and Vador, Enor. Units

\begin{tabular}{|c|c|c|c|c|c|c|c|c|c|c|c|}
\hline $\begin{array}{c}\text { Temo. } \\
\text { R }\end{array}$ & $\begin{array}{c}\text { Pressure } \\
\text { Dsl. }\end{array}$ & $\begin{array}{l}\text { Density } \\
\text { lb/et**3 }\end{array}$ & $\begin{array}{l}\text { Thernal } \\
\text { Cond. } \\
\text { Brulfteh.R }\end{array}$ & $\begin{array}{c}\text { Viscosity } \\
\text { mlero- } \\
\text { Itiftes }\end{array}$ & $\begin{array}{c}\text { Thermal } \\
\text { oiffus Ivity } \\
\text { et } \neq \neq 2 / h\end{array}$ & $\begin{array}{c}\text { Tenp. } \\
R\end{array}$ & $\begin{array}{c}\text { Pressure } \\
\text { osia }\end{array}$ & $\begin{array}{l}\text { Denslty } \\
\mid b / f t * \$ 3\end{array}$ & $\begin{array}{l}\text { Thermal } \\
\text { Cond. } \\
\text { Btulfteh.R }\end{array}$ & $\begin{array}{c}\text { viscosity } \\
\text { micro- } \\
\text { Ib/ftes }\end{array}$ & $\begin{array}{c}\text { Thermal } \\
\text { DIf fuslvity } \\
\text { ft* } * 2 / \mathrm{h}\end{array}$ \\
\hline $\begin{array}{l}150 . \\
150 .\end{array}$ & $\begin{array}{l}6.727 \\
6.727\end{array}$ & $\begin{array}{r}73.3293 \\
.1364\end{array}$ & $\begin{array}{l}.09225 \\
.00429\end{array}$ & $\begin{array}{r}255.20 \\
4.12\end{array}$ & $\begin{array}{l}.00313 \\
.13819\end{array}$ & $\begin{array}{l}242 . \\
242 .\end{array}$ & $\begin{array}{l}314.410 \\
314.410\end{array}$ & $\begin{array}{r}54.0451 \\
5.4150\end{array}$ & $\begin{array}{l}.04932 \\
.00867\end{array}$ & $\begin{array}{r}47.32 \\
7.49\end{array}$ & $\begin{array}{l}.00164 \\
.00376\end{array}$ \\
\hline $\begin{array}{l}152 . \\
152 .\end{array}$ & $\begin{array}{l}7.707 \\
7.707\end{array}$ & $\begin{array}{r}72.9867 \\
.1546\end{array}$ & $\begin{array}{l}.0 \$ 137 \\
.00435\end{array}$ & $\begin{array}{r}150.90 \\
4.18\end{array}$ & $\begin{array}{r}.00311 \\
.12337\end{array}$ & $\begin{array}{l}244 . \\
244 .\end{array}$ & $\begin{array}{l}331.098 \\
331.098\end{array}$ & $\begin{array}{r}53.4307 \\
5.7440\end{array}$ & $\begin{array}{r}.04830 \\
.00887\end{array}$ & $\begin{array}{r}46.02 \\
7.61\end{array}$ & $\begin{array}{l}.00159 \\
.00349\end{array}$ \\
\hline $\begin{array}{l}154 . \\
154 .\end{array}$ & $\begin{array}{l}8.795 \\
8.795\end{array}$ & $\begin{array}{r}72.6513 \\
.1745\end{array}$ & $\begin{array}{l}.09049 \\
.00442\end{array}$ & $\begin{array}{r}146.74 \\
4.25\end{array}$ & $\begin{array}{l}.00309 \\
.11050\end{array}$ & $\begin{array}{l}240 . \\
246 .\end{array}$ & $\begin{array}{l}348.414 \\
348.414\end{array}$ & $\begin{array}{r}52.7969 \\
6.0937\end{array}$ & $\begin{array}{l}.04726 \\
.00909\end{array}$ & $\begin{array}{r}44.73 \\
7.73\end{array}$ & $\begin{array}{l}.00153 \\
.00323\end{array}$ \\
\hline $\begin{array}{l}153 . \\
253 .\end{array}$ & $\begin{array}{l}10.001 \\
10.001\end{array}$ & $\begin{array}{r}72.3140 \\
.1963\end{array}$ & $\begin{array}{l}.08961 \\
.00449\end{array}$ & $\begin{array}{r}142.72 \\
4.31\end{array}$ & $\begin{array}{l}.00307 \\
.09926\end{array}$ & $\begin{array}{l}248 . \\
248 .\end{array}$ & $\begin{array}{l}366.375 \\
365.375\end{array}$ & $\begin{array}{r}52.2419 \\
0.4659\end{array}$ & $\begin{array}{l}.04623 \\
.00932\end{array}$ & $\begin{array}{r}43.45 \\
7.86\end{array}$ & $\begin{array}{l}.00148 \\
.00299\end{array}$ \\
\hline $\begin{array}{l}158 . \\
158 .\end{array}$ & $\begin{array}{l}11.332 \\
11.332\end{array}$ & $\begin{array}{r}71.9748 \\
.2202\end{array}$ & $\begin{array}{l}.08873 \\
.00456\end{array}$ & $\begin{array}{r}138.83 \\
4.37\end{array}$ & $\begin{array}{l}.00305 \\
.08943\end{array}$ & $\begin{array}{l}250 . \\
250 .\end{array}$ & $\begin{array}{l}384.997 \\
384.997\end{array}$ & $\begin{array}{r}51.4834 \\
6.8631\end{array}$ & $\begin{array}{l}.04519 \\
.00958\end{array}$ & $\begin{array}{r}42.18 \\
7.99\end{array}$ & $\begin{array}{l}.00142 \\
.00275\end{array}$ \\
\hline $\begin{array}{l}160 . \\
160 .\end{array}$ & $\begin{array}{l}12.797 \\
12.797\end{array}$ & $\begin{array}{r}71.6336 \\
.2462\end{array}$ & $\begin{array}{l}.08786 \\
.00463\end{array}$ & $\begin{array}{r}135.07 \\
4.43\end{array}$ & $\begin{array}{l}.00303 \\
.08079\end{array}$ & $\begin{array}{l}252 . \\
252 .\end{array}$ & $\begin{array}{l}404.297 \\
404.297\end{array}$ & $\begin{array}{r}50.7590 \\
7.2879\end{array}$ & $\begin{array}{l}.04416 \\
.00986\end{array}$ & $\begin{array}{r}40.92 \\
8.13\end{array}$ & $\begin{array}{l}.00136 \\
.00253\end{array}$ \\
\hline $\begin{array}{l}162 . \\
162 .\end{array}$ & $\begin{array}{l}14.404 \\
14.404\end{array}$ & $\begin{array}{r}71.2902 \\
.2744\end{array}$ & $\begin{array}{l}.08698 \\
.00470\end{array}$ & $\begin{array}{r}131.44 \\
4.49\end{array}$ & $\begin{array}{l}.00301 \\
.07317\end{array}$ & $\begin{array}{l}254 . \\
234 .\end{array}$ & $\begin{array}{l}424.292 \\
424.292\end{array}$ & $\begin{array}{r}50.0254 \\
7.7436\end{array}$ & $\begin{array}{l}.04313 \\
.01019\end{array}$ & $\begin{array}{r}39.65 \\
8.29\end{array}$ & $\begin{array}{l}.00129 \\
.00232\end{array}$ \\
\hline $\begin{array}{l}164 . \\
164 .\end{array}$ & $\begin{array}{l}16.163 \\
16.163\end{array}$ & $\begin{array}{r}70.9448 \\
.3051\end{array}$ & $\begin{array}{l}.08610 \\
.00477\end{array}$ & $\begin{array}{r}127.93 \\
4.55\end{array}$ & $\begin{array}{l}.00299 \\
.06643\end{array}$ & $\begin{array}{l}256 . \\
256 .\end{array}$ & $\begin{array}{l}445.001 \\
445.001\end{array}$ & $\begin{array}{r}48.2589 \\
8.2341\end{array}$ & $\begin{array}{l}.04220 \\
.01055\end{array}$ & $\begin{array}{r}38.39 \\
8.45\end{array}$ & $\begin{array}{l}.00123 \\
.00212\end{array}$ \\
\hline $\begin{array}{l}166 . \\
166 .\end{array}$ & $\begin{array}{l}18.083 \\
18.083\end{array}$ & $\begin{array}{r}70.5971 \\
.3382\end{array}$ & $\begin{array}{l}.08521 \\
.00484\end{array}$ & $\begin{array}{r}124.54 \\
4.51\end{array}$ & $\begin{array}{l}.00297 \\
.06046\end{array}$ & $\begin{array}{l}258 . \\
258 .\end{array}$ & $\begin{array}{l}466.444 \\
466.444\end{array}$ & $\begin{array}{r}48.4548 \\
8.7645\end{array}$ & $\begin{array}{l}.04109 \\
.01096\end{array}$ & $\begin{array}{r}37.12 \\
8.63\end{array}$ & $\begin{array}{l}.00116 \\
.00192\end{array}$ \\
\hline $\begin{array}{l}168 . \\
168 .\end{array}$ & $\begin{array}{l}20.173 \\
20.173\end{array}$ & $\begin{array}{r}70.2471 \\
.3741\end{array}$ & $\begin{array}{l}.08433 \\
.00491\end{array}$ & $\begin{array}{r}121.25 \\
4.67\end{array}$ & $\begin{array}{l}.00295 \\
.05514\end{array}$ & $\begin{array}{l}260 . \\
260 .\end{array}$ & $\begin{array}{l}488.642 \\
488.642\end{array}$ & $\begin{array}{r}47.6069 \\
9.3410\end{array}$ & $\begin{array}{l}.04009 \\
.01245\end{array}$ & $\begin{array}{r}35.85 \\
8.82\end{array}$ & $\begin{array}{l}.00109 \\
.00174\end{array}$ \\
\hline $\begin{array}{l}170 . \\
170 .\end{array}$ & $\begin{array}{l}22.443 \\
22.443\end{array}$ & $\begin{array}{r}69.0966 \\
.4127\end{array}$ & $\begin{array}{l}.08344 \\
.00498\end{array}$ & $\begin{array}{r}118.07 \\
4.74\end{array}$ & $\begin{array}{l}.00293 \\
.05040\end{array}$ & $\begin{array}{l}262 . \\
262 .\end{array}$ & $\begin{array}{l}511.619 \\
511.619\end{array}$ & $\begin{array}{r}46.7073 \\
9.9717\end{array}$ & $\begin{array}{l}.03912 \\
.01201\end{array}$ & $\begin{array}{r}34.55 \\
9.03\end{array}$ & $\begin{array}{l}.00102 \\
.00156\end{array}$ \\
\hline $\begin{array}{l}172 . \\
172 .\end{array}$ & $\begin{array}{l}24.903 \\
24.903\end{array}$ & $\begin{array}{r}69.5396 \\
.4542\end{array}$ & $\begin{array}{l}.08256 \\
.00505\end{array}$ & $\begin{array}{r}115.00 \\
4.80\end{array}$ & $\begin{array}{l}.00290 \\
.04015\end{array}$ & $\begin{array}{l}264 . \\
264 .\end{array}$ & $\begin{array}{l}335.399 \\
535.399\end{array}$ & $\begin{array}{l}45.7454 \\
10.6676\end{array}$ & $\begin{array}{l}.03818 \\
.01268\end{array}$ & $\begin{array}{r}33.24 \\
9.26\end{array}$ & $\begin{array}{l}.00094 \\
.00138\end{array}$ \\
\hline $\begin{array}{l}174 . \\
174 .\end{array}$ & $\begin{array}{l}27.562 \\
27.562\end{array}$ & $\begin{array}{r}69.1821 \\
.4988\end{array}$ & $\begin{array}{l}.08167 \\
.00513\end{array}$ & $\begin{array}{r}112.02 \\
4.86\end{array}$ & $\begin{array}{l}.00288 \\
.04234\end{array}$ & $\begin{array}{l}266 . \\
266 .\end{array}$ & $\begin{array}{l}560.010 \\
560.010\end{array}$ & $\begin{array}{l}44.7061 \\
11.4439\end{array}$ & $\begin{array}{l}.03730 \\
.01349\end{array}$ & $\begin{array}{r}32.88 \\
9.52\end{array}$ & $\begin{array}{l}.00085 \\
.00120\end{array}$ \\
\hline $\begin{array}{l}176 . \\
176 .\end{array}$ & $\begin{array}{l}30.431 \\
30.431\end{array}$ & $\begin{array}{r}68.0217 \\
.5466\end{array}$ & $\begin{array}{l}.08077 \\
.00520\end{array}$ & $\begin{array}{r}109.14 \\
4.92\end{array}$ & $\begin{array}{l}.00285 \\
.03892\end{array}$ & $\begin{array}{l}268 . \\
268 .\end{array}$ & $\begin{array}{l}585.483 \\
585.483\end{array}$ & $\begin{array}{l}43.5671 \\
12.3231\end{array}$ & $\begin{array}{l}.03651 \\
.01449\end{array}$ & $\begin{array}{r}30.48 \\
9.83\end{array}$ & $\begin{array}{l}.00076 \\
.00103\end{array}$ \\
\hline $\begin{array}{l}178 . \\
178 .\end{array}$ & $\begin{array}{l}33.519 \\
33.519\end{array}$ & $\begin{array}{r}68.4586 \\
.5978\end{array}$ & $\begin{array}{l}.07988 \\
.00528\end{array}$ & $\begin{array}{r}106.34 \\
4.99\end{array}$ & $\begin{array}{l}.00283 \\
.03583\end{array}$ & $\begin{array}{l}270 . \\
270 .\end{array}$ & $\begin{array}{l}611.855 \\
611.855\end{array}$ & $\begin{array}{l}42.2934 \\
13.3407\end{array}$ & $\begin{array}{l}.03583 \\
.02576\end{array}$ & $\begin{array}{l}29.00 \\
10.18\end{array}$ & $\begin{array}{l}.00066 \\
.00085\end{array}$ \\
\hline $\begin{array}{l}180 . \\
180 .\end{array}$ & $\begin{array}{l}36.838 \\
36.838\end{array}$ & $\begin{array}{r}68.0925 \\
.6525\end{array}$ & $\begin{array}{l}.07898 \\
.00535\end{array}$ & $\begin{array}{r}103.64 \\
5.05\end{array}$ & $\begin{array}{l}.00280 \\
.03304\end{array}$ & $\begin{array}{l}272 \\
272\end{array}$ & $\begin{array}{l}639.170 \\
639.170\end{array}$ & $\begin{array}{l}40.8236 \\
14.5588\end{array}$ & $\begin{array}{l}.03533 \\
.01744\end{array}$ & $\begin{array}{l}27.39 \\
10.62\end{array}$ & $\begin{array}{l}.00055 \\
.00067\end{array}$ \\
\hline $\begin{array}{l}282 . \\
18 ? .\end{array}$ & $\begin{array}{l}40.397 \\
40.397\end{array}$ & $\begin{array}{r}67.7232 \\
.7109\end{array}$ & $\begin{array}{l}.07808 \\
.00543\end{array}$ & $\begin{array}{r}101.01 \\
5.11\end{array}$ & $\begin{array}{l}.00276 \\
.03052\end{array}$ & $\begin{array}{l}274 \\
274\end{array}$ & $\begin{array}{l}667.486 \\
567.486\end{array}$ & $\begin{array}{l}39.0338 \\
16.1040\end{array}$ & $\begin{array}{l}.03511 \\
.01975\end{array}$ & $\begin{array}{l}25.57 \\
11.20\end{array}$ & $\begin{array}{l}.00042 \\
.00048\end{array}$ \\
\hline $\begin{array}{l}184 . \\
184 .\end{array}$ & $\begin{array}{l}44.207 \\
44.207\end{array}$ & $\begin{array}{r}67.3508 \\
.7732\end{array}$ & $\begin{array}{l}.07718 \\
.00551\end{array}$ & $\begin{array}{r}98.47 \\
5.18\end{array}$ & $\begin{array}{l}.00275 \\
.02822\end{array}$ & $\begin{array}{l}276 . \\
276 .\end{array}$ & $\begin{array}{l}696.886 \\
696.886\end{array}$ & $\begin{array}{l}36.5919 \\
18.3235\end{array}$ & $\begin{array}{l}.03533 \\
.02438\end{array}$ & $\begin{array}{l}23.29 \\
12.08\end{array}$ & $\begin{array}{l}.00025 \\
.00027\end{array}$ \\
\hline $\begin{array}{l}186 . \\
186 .\end{array}$ & $\begin{array}{l}48.279 \\
48.279\end{array}$ & $\begin{array}{r}06.9749 \\
.8394\end{array}$ & $\begin{array}{l}.07627 \\
.00558\end{array}$ & $\begin{array}{r}96.00 \\
5.24\end{array}$ & $\begin{array}{l}.00272 \\
.02613\end{array}$ & $\begin{array}{l}278 . \\
278 .\end{array}$ & $\begin{array}{l}727.543 \\
727.543\end{array}$ & $\begin{array}{l}31.3822 \\
23.9567\end{array}$ & $\begin{array}{l}.05145 \\
.06158\end{array}$ & $\begin{array}{l}19.16 \\
14.65\end{array}$ & $\begin{array}{l}.00005 \\
.00003\end{array}$ \\
\hline $\begin{array}{l}188 . \\
188 .\end{array}$ & $\begin{array}{l}52.623 \\
32.623\end{array}$ & $\begin{array}{r}66.5956 \\
.9099\end{array}$ & $\begin{array}{l}.07536 \\
.00565\end{array}$ & $\begin{array}{r}93.60 \\
5.31\end{array}$ & $\begin{array}{l}.00270 \\
.02423\end{array}$ & $\begin{array}{l}278.246 \\
278.246\end{array}$ & $\begin{array}{l}731.426 \\
731.426\end{array}$ & $\begin{array}{l}27.2276 \\
27.2276\end{array}$ & & & \\
\hline
\end{tabular}


Tanle 7. Iransoort froperties of Exyoon, Isobars, Fngr. Units.

e. esla Isntar

10. DSI a Iscber

\begin{tabular}{|c|c|c|c|c|c|c|c|c|c|}
\hline TPme. & $\begin{array}{l}\text { nonsity } \\
\text { InIf+**3 }\end{array}$ & $\begin{array}{l}\text { Theptal V } \\
\text { Cond. } \\
\text { gruleten.R }\end{array}$ & $\begin{array}{c}\text { Visensity } \\
\text { ricro- } \\
\text { Inift.s }\end{array}$ & $\begin{array}{c}\text { Thermal } \\
\text { Difusivity } \\
\text { ft**2/h }\end{array}$ & TeTo. & $10 / f t * * 3$ & $\begin{array}{l}\text { Thermal } \\
\text { Cond. } \\
\text { BTulft.h.R }\end{array}$ & $\begin{array}{c}\text { Viscosity } \\
\text { mlero- } \\
\text { lo/et.s }\end{array}$ & $\begin{array}{c}\text { Thermal } \\
\text { olffusivity } \\
\text { ft**2/n }\end{array}$ \\
\hline 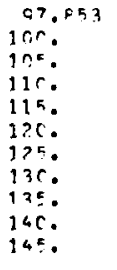 & $\begin{array}{l}81.564 ? \\
H 1.2192 \\
46.0548 \\
79.4914 \\
7 F .0 ? 00 \\
79.1446 \\
77.3415 \\
74.5709 \\
75.7721 \\
74.7644 \\
74.1470\end{array}$ & $\begin{array}{l}.11770 \\
.11940 \\
.11354 \\
.11084 \\
.10294 \\
.10594 \\
.10347 \\
.10116 \\
.06099 \\
.09456 \\
.00445\end{array}$ & $\begin{array}{l}324.13 \\
317.62 \\
297.53 \\
277.54 \\
259.49 \\
249.28 \\
223.21 \\
207.23 \\
192.64 \\
179.09 \\
166.43\end{array}$ & $\begin{array}{l}.00343 \\
.00340 \\
.00354 \\
.00349 \\
.00344 \\
.00340 \\
.00335 \\
.00331 \\
.00327 \\
.00322 \\
.00318\end{array}$ & $\begin{array}{l}97.850 \\
100 . \\
105 . \\
110 . \\
115 . \\
129 . \\
125 . \\
130 . \\
135 . \\
140 . \\
145 .\end{array}$ & $\begin{array}{l}41.5656 \\
91.2207 \\
80.4594 \\
79.5941 \\
79.9238 \\
78.18 .75 \\
77.3644 \\
75.5742 \\
75.7756 \\
74.9381 \\
74.1500\end{array}$ & $\begin{array}{l}.11770 \\
.11641 \\
.11355 \\
.11085 \\
.10830 \\
.10585 \\
.10348 \\
.10117 \\
.09891 \\
.09667 \\
.09446\end{array}$ & $\begin{array}{l}326.20 \\
317.71 \\
297.62 \\
277.74 \\
258.56 \\
240.35 \\
323.28 \\
207.40 \\
192.70 \\
179.15 \\
169.69\end{array}$ & $\begin{array}{l}.00363 \\
.00360 \\
.00354 \\
.00349 \\
.00344 \\
.00340 \\
.00335 \\
.00331 \\
.00327 \\
.00322 \\
.00318\end{array}$ \\
\hline $\begin{array}{l}145 .+20 \\
145.928 \\
150 . \\
1550 \\
160 . \\
1450 \\
170 \\
1750 \\
1950 \\
1950 \\
169 . \\
1550\end{array}$ & $\begin{array}{r}74.0104 \\
.1027 \\
.1009 \\
.0075 \\
.0243 \\
.0713 \\
.0924 \\
.0090 \\
.0935 \\
.0919 \\
.0790 \\
.0770\end{array}$ & $\begin{array}{l}.09408 \\
.00415 \\
.00428 \\
.00443 \\
.00458 \\
.00474 \\
.00499 \\
.00505 \\
.005 ? 1 \\
.00527 \\
.0055 ? \\
.00540\end{array}$ & $\begin{array}{r}164.47 \\
4.00 \\
4.12 \\
4.27 \\
4.41 \\
4.56 \\
4.71 \\
4.85 \\
5.00 \\
5.14 \\
5.29 \\
5.43\end{array}$ & $\begin{array}{l}.00317 \\
.17700 \\
.18960 \\
.20294 \\
.21778 \\
.23314 \\
.24901 \\
.26540 \\
.28231 \\
.20973 \\
.31766 \\
.33610\end{array}$ & $\begin{array}{l}150 . \\
155 . \\
+155.999 \\
+155.908 \\
150 . \\
165 . \\
170 . \\
175 . \\
189 . \\
185 . \\
190 . \\
195 .\end{array}$ & $\begin{array}{r}73.3230 \\
72.4834 \\
72.3143 \\
.1933 \\
.1910 \\
.1848 \\
.1790 \\
.1736 \\
.1685 \\
.1637 \\
.1592 \\
.1549\end{array}$ & $\begin{array}{l}.09225 \\
.09005 \\
.08961 \\
.00449 \\
.00461 \\
.00477 \\
.00492 \\
.00508 \\
.00523 \\
.00539 \\
.00555 \\
.00571\end{array}$ & $\begin{array}{r}155.23 \\
144.72 \\
142.72 \\
4.31 \\
4.42 \\
4.57 \\
4.71 \\
4.86 \\
5.00 \\
5.15 \\
5.29 \\
5.44\end{array}$ & $\begin{array}{l}.00313 \\
.00308 \\
.00304 \\
.09927 \\
.10540 \\
.11327 \\
.12138 \\
.12973 \\
.13833 \\
.14717 \\
.15626 \\
.16559\end{array}$ \\
\hline $\begin{array}{l}200 . \\
205 \\
210 \\
2150 \\
220 \\
? 250 \\
230 \\
2350 \\
240 \\
745\end{array}$ & $\begin{array}{l}.0750 \\
.0721 \\
.0714 \\
.0597 \\
.0481 \\
.0645 \\
.0451 \\
.0437 \\
.0523 \\
.0411\end{array}$ & $\begin{array}{l}. \cos 55 \\
. \cos 11 \\
.00517 \\
.00534 \\
.00550 \\
.0 \cosh \\
.006 R 2 \\
.00598 \\
.00713 \\
.00739\end{array}$ & $\begin{array}{l}5.57 \\
5.71 \\
5.86 \\
6.00 \\
6.14 \\
6.78 \\
6.47 \\
0.53 \\
5.70 \\
6.84\end{array}$ & $\begin{array}{l}.35503 \\
.37445 \\
.39434 \\
.41471 \\
.43554 \\
.45683 \\
.47854 \\
.50073 \\
.52334 \\
.54638\end{array}$ & $\begin{array}{l}200 . \\
205 . \\
210 . \\
215 . \\
220 . \\
225 . \\
230 . \\
235 . \\
240 . \\
245 .\end{array}$ & $\begin{array}{l}.1509 \\
.1471 \\
.1435 \\
.1401 \\
.1368 \\
.1337 \\
.1307 \\
.1279 \\
.1251 \\
.1225\end{array}$ & $\begin{array}{l}.00587 \\
.00603 \\
.00620 \\
.00636 \\
.00652 \\
.00668 \\
.00684 \\
.00699 \\
.00715 \\
.00731\end{array}$ & $\begin{array}{l}5.58 \\
5.72 \\
5.86 \\
6.01 \\
5.15 \\
6.20 \\
6.43 \\
6.57 \\
5.71 \\
6.94\end{array}$ & $\begin{array}{l}.17516 \\
.18496 \\
.19500 \\
.20527 \\
.21576 \\
.22648 \\
.23741 \\
.24857 \\
.25993 \\
.27151\end{array}$ \\
\hline $\begin{array}{l}370 . \\
310 . \\
320 . \\
330 . \\
340 . \\
350 \\
350 \\
370 \\
380 . \\
350 .\end{array}$ & $\begin{array}{l}.0498 \\
.0482 \\
.0467 \\
.0452 \\
.0439 \\
.0426 \\
.0415 \\
.0403 \\
.0393 \\
.0383\end{array}$ & $\begin{array}{l}.00896 \\
.00925 \\
.00953 \\
.00781 \\
.01009 \\
.01035 \\
.01054 \\
.01091 \\
.01117 \\
.01144\end{array}$ & $\begin{array}{r}8.31 \\
8.87 \\
8.83 \\
9.09 \\
9.34 \\
9.59 \\
9.83 \\
10.08 \\
10.32 \\
10.55\end{array}$ & $\begin{array}{r}.82512 \\
.98030 \\
.93694 \\
.99499 \\
1.05442 \\
1.11519 \\
1.17728 \\
1.24069 \\
1.30539 \\
1.37137\end{array}$ & $\begin{array}{l}300 . \\
310 . \\
320 . \\
330 . \\
340 . \\
350 . \\
360 . \\
370 . \\
380 . \\
390 .\end{array}$ & $\begin{array}{l}.0998 \\
.0965 \\
.0935 \\
.0906 \\
.0879 \\
.0854 \\
.0830 \\
.0807 \\
.0784 \\
.0766\end{array}$ & $\begin{array}{l}.00897 \\
.00926 \\
.00954 \\
.00982 \\
.01010 \\
.01038 \\
.01065 \\
.01092 \\
.01118 \\
.01145\end{array}$ & $\begin{array}{r}8.32 \\
8.58 \\
8.84 \\
9.09 \\
9.34 \\
9.59 \\
9.84 \\
10.08 \\
10.32 \\
10.56\end{array}$ & $\begin{array}{l}.41138 \\
.43903 \\
.46742 \\
.49650 \\
.52627 \\
.55672 \\
.58782 \\
.61958 \\
.65198 \\
.68502\end{array}$ \\
\hline $\begin{array}{l}400 . \\
410 . \\
430 . \\
430 \\
440 . \\
450 . \\
450 . \\
470 \\
480 . \\
490 .\end{array}$ & $\begin{array}{l}.0373 \\
.0364 \\
.0355 \\
.0347 \\
.0339 \\
.0321 \\
.0324 \\
.0317 \\
.0311 \\
.0304\end{array}$ & $\begin{array}{l}.01170 \\
.01193 \\
.01222 \\
.01247 \\
.01273 \\
.01298 \\
.01323 \\
.01348 \\
.01373 \\
.01397\end{array}$ & $\begin{array}{l}10.79 \\
11.02 \\
11.25 \\
11.48 \\
11.71 \\
11.93 \\
12.16 \\
12.37 \\
12.59 \\
12.81\end{array}$ & $\begin{array}{l}1.43861 \\
1.50710 \\
1.57683 \\
1.64779 \\
1.71996 \\
1.79332 \\
1.85787 \\
1.94359 \\
2.02046 \\
2.00847\end{array}$ & $\begin{array}{l}400 . \\
410 . \\
420 . \\
430 . \\
440 . \\
450 . \\
450 . \\
470 . \\
490 . \\
490 .\end{array}$ & $\begin{array}{l}.0747 \\
.0728 \\
.0711 \\
.0694 \\
.0678 \\
.0663 \\
.0549 \\
.0635 \\
.0622 \\
.0609\end{array}$ & $\begin{array}{l}.01171 \\
.01197 \\
.01223 \\
.01248 \\
.01274 \\
.01299 \\
.01324 \\
.01349 \\
.011374 \\
.01399\end{array}$ & $\begin{array}{l}10.79 \\
11.03 \\
11.26 \\
11.49 \\
11.71 \\
11.94 \\
12.16 \\
12.38 \\
12.80 \\
12.81\end{array}$ & $\begin{array}{r}.71869 \\
.75298 \\
.78790 \\
.82342 \\
.85955 \\
.89628 \\
.93360 \\
.97150 \\
1.00998 \\
1.04903\end{array}$ \\
\hline $\begin{array}{l}500 . \\
510 . \\
520 . \\
530 . \\
540 .\end{array}$ & $\begin{array}{l}.0298 \\
.0292 \\
.0787 \\
.0781 \\
.0276\end{array}$ & $\begin{array}{l}.01472 \\
.01446 \\
.01471 \\
.01495 \\
.01519\end{array}$ & $\begin{array}{l}13.02 \\
13.23 \\
13.44 \\
13.65 \\
13.86\end{array}$ & $\begin{array}{l}2.17761 \\
2.25784 \\
2.33917 \\
2.42157 \\
2.50501\end{array}$ & $\begin{array}{l}500 . \\
510 . \\
520 . \\
530 . \\
540 .\end{array}$ & $\begin{array}{l}.0597 \\
.0585 \\
.0574 \\
.0563 \\
.0552\end{array}$ & $\begin{array}{l}.01423 \\
.01448 \\
.01472 \\
.01496 \\
.01520\end{array}$ & $\begin{array}{l}13.02 \\
13.24 \\
13.45 \\
13.65 \\
13.86\end{array}$ & $\begin{array}{l}1.08864 \\
1.12880 \\
1.16950 \\
1.21074 \\
1.25250\end{array}$ \\
\hline
\end{tabular}

* Two Phase Boundary 
Table 7. Transport Properties of Dxyoen, Isobars, Engr. Units.

15. DSIa Isobar

Teno. Density Thermal Viscosity prermal
conde

20. psla I sobar

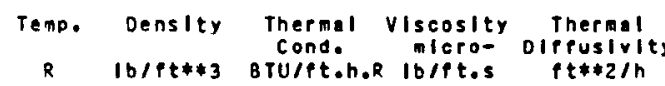

\begin{tabular}{|c|c|c|c|c|c|c|c|c|c|}
\hline $\begin{array}{l}97.8 \in B \\
100 . \\
105 . \\
110 . \\
115 . \\
120 . \\
125 . \\
130 . \\
135 . \\
140 . \\
145 .\end{array}$ & $\begin{array}{l}81.5470 \\
81.2232 \\
60.4620 \\
79.6968 \\
78.9266 \\
78.1505 \\
77.3677 \\
76.5774 \\
75.7791 \\
74.9718 \\
74.1547\end{array}$ & $\begin{array}{l}.11771 \\
.11642 \\
.11356 \\
.11086 \\
.10831 \\
.10586 \\
.10349 \\
.10118 \\
.09892 \\
.09668 \\
.09447\end{array}$ & $\begin{array}{l}326.27 \\
317.81 \\
297.71 \\
277.82 \\
258.63 \\
240.42 \\
223.35 \\
207.46 \\
192.76 \\
179.21 \\
166.74\end{array}$ & $\begin{array}{l}.00363 \\
.00360 \\
.00354 \\
.00349 \\
.00344 \\
.00340 \\
.00335 \\
.00331 \\
.00327 \\
.00323 \\
.00318\end{array}$ & $\begin{array}{l}* 97.875 \\
100 . \\
105 . \\
110 . \\
115 . \\
120 . \\
125 . \\
130 . \\
135 . \\
140 . \\
145 .\end{array}$ & $\begin{array}{l}81.5484 \\
81.2257 \\
80.4646 \\
79.6995 \\
78.9294 \\
78.1534 \\
77.3708 \\
76.5807 \\
75.7825 \\
74.9754 \\
74.1586\end{array}$ & $\begin{array}{l}.11771 \\
.11643 \\
.11357 \\
.11087 \\
.10832 \\
.10587 \\
.10350 \\
.10119 \\
.09893 \\
.09670 \\
.09448\end{array}$ & $\begin{array}{l}326.34 \\
317.91 \\
297.80 \\
277.90 \\
258.71 \\
240.49 \\
223.41 \\
207.52 \\
192.82 \\
179.26 \\
166.79\end{array}$ & $\begin{array}{l}.00363 \\
.00360 \\
.00354 \\
.00349 \\
.00344 \\
.00340 \\
.00335 \\
.00331 \\
.00327 \\
.00323 \\
.00318\end{array}$ \\
\hline $\begin{array}{l}150 . \\
155 . \\
160 . \\
162.698 \\
162.698 \\
162.6 \\
165 . \\
170 . \\
175 . \\
180 . \\
185 . \\
190 . \\
195 .\end{array}$ & $\begin{array}{r}73.3271 \\
72.4878 \\
71.6356 \\
71.1700 \\
.2848 \\
.2804 \\
.2713 \\
.2628 \\
.2549 \\
.2475 \\
.2406 \\
.2340\end{array}$ & $\begin{array}{l}.09227 \\
.09007 \\
.08786 \\
.08667 \\
.00472 \\
.00479 \\
.00495 \\
.00510 \\
.00526 \\
.00542 \\
.00557 \\
.00573\end{array}$ & $\begin{array}{r}155.29 \\
144.76 \\
135.09 \\
130.20 \\
4.51 \\
4.58 \\
4.72 \\
4.87 \\
5.01 \\
5.16 \\
5.30 \\
5.44\end{array}$ & $\begin{array}{l}.00313 \\
.00309 \\
.00303 \\
.00301 \\
.07073 \\
.07322 \\
.07875 \\
.08442 \\
.09026 \\
.09624 \\
.10239 \\
.10868\end{array}$ & $\begin{array}{l}150 . \\
155 . \\
160 . \\
165 . \\
167.840 \\
+167.840 \\
170 . \\
175 . \\
180 . \\
185 . \\
190 . \\
195 .\end{array}$ & $\begin{array}{r}73.3312 \\
72.4922 \\
71.6403 \\
70.7741 \\
70.2751 \\
.3711 \\
.3657 \\
.3539 \\
.3430 \\
.3328 \\
.3232 \\
.3142\end{array}$ & $\begin{array}{l}.09228 \\
.09008 \\
.08788 \\
.08566 \\
.08440 \\
.00490 \\
.00497 \\
.00512 \\
.00528 \\
.00544 \\
.00559 \\
.00575\end{array}$ & $\begin{array}{r}155.34 \\
144.81 \\
135.14 \\
126.25 \\
121.51 \\
4.67 \\
4.73 \\
4.88 \\
5.02 \\
5.17 \\
5.31 \\
5.45\end{array}$ & $\begin{array}{l}.00313 \\
.00309 \\
.00304 \\
.00298 \\
.00295 \\
.05554 \\
.05738 \\
.06173 \\
.06618 \\
.07074 \\
.07541 \\
.08019\end{array}$ \\
\hline $\begin{array}{l}200 . \\
205 \\
210 \\
215 . \\
220 . \\
225 . \\
230 \\
235 . \\
240 . \\
245 .\end{array}$ & $\begin{array}{l}.2278 \\
.2220 \\
.2164 \\
.2112 \\
.2062 \\
.2014 \\
.1968 \\
.1925 \\
.1884 \\
.1844\end{array}$ & $\begin{array}{l}.00589 \\
.00605 \\
.00621 \\
.00637 \\
.00653 \\
.00669 \\
.00685 \\
.00701 \\
.00717 \\
.00733\end{array}$ & $\begin{array}{l}5.59 \\
5.73 \\
5.87 \\
6.01 \\
6.15 \\
6.29 \\
6.43 \\
6.57 \\
6.71 \\
6.85\end{array}$ & $\begin{array}{l}.11513 \\
.12174 \\
.12849 \\
.13539 \\
.14244 \\
.14964 \\
.15697 \\
.16445 \\
.17207 \\
.17903\end{array}$ & $\begin{array}{l}200 . \\
205 . \\
210 . \\
215 . \\
220 . \\
225 . \\
230 . \\
235 . \\
240 . \\
245 .\end{array}$ & $\begin{array}{l}.3057 \\
.2977 \\
.2901 \\
.2830 \\
.2762 \\
.2697 \\
.2636 \\
.2577 \\
.2521 \\
.2467\end{array}$ & $\begin{array}{l}.00591 \\
.00607 \\
.00623 \\
.00639 \\
.00655 \\
.00671 \\
.00687 \\
.00703 \\
.00718 \\
.00734\end{array}$ & $\begin{array}{l}5.59 \\
5.74 \\
5.88 \\
6.02 \\
6.16 \\
6.30 \\
6.44 \\
6.58 \\
6.72 \\
6.86\end{array}$ & $\begin{array}{l}.08509 \\
.09009 \\
.09520 \\
.10043 \\
.10575 \\
.11119 \\
.11673 \\
.12237 \\
.12812 \\
.13397\end{array}$ \\
\hline $\begin{array}{l}250 . \\
255 . \\
260 . \\
265 . \\
270 . \\
275 . \\
280 . \\
2850 \\
290 . \\
295 .\end{array}$ & $\begin{array}{l}.1806 \\
.1770 \\
.1735 \\
.1701 \\
.1669 \\
.1638 \\
.1608 \\
.1580 \\
.1552 \\
.1525\end{array}$ & $\begin{array}{l}.00748 \\
.00764 \\
.00779 \\
.00795 \\
.00810 \\
.00826 \\
.00841 \\
.00856 \\
.00870 \\
.00884\end{array}$ & $\begin{array}{l}6.99 \\
7.12 \\
7.26 \\
7.40 \\
7.53 \\
7.66 \\
7.80 \\
7.93 \\
8.06 \\
6.19\end{array}$ & $\begin{array}{l}.18773 \\
.19577 \\
.20395 \\
.21228 \\
.22076 \\
.22942 \\
.23812 \\
.24670 \\
.25546 \\
.26437\end{array}$ & $\begin{array}{l}250 . \\
255 . \\
260 . \\
265 . \\
270 . \\
275 . \\
280 . \\
285 . \\
290 . \\
295 .\end{array}$ & $\begin{array}{l}.2416 \\
.2367 \\
.2320 \\
.2275 \\
.2231 \\
.2190 \\
.2149 \\
.2111 \\
.2073 \\
.2037\end{array}$ & $\begin{array}{l}.00750 \\
.00765 \\
.00781 \\
.00796 \\
.00812 \\
.00828 \\
.00843 \\
.00857 \\
.00871 \\
.00886\end{array}$ & $\begin{array}{l}6.99 \\
7.13 \\
7.27 \\
7.40 \\
7.54 \\
7.67 \\
7.80 \\
7.94 \\
8.07 \\
8.20\end{array}$ & $\begin{array}{r}13993 \\
.14599 \\
.15215 \\
.15842 \\
.16481 \\
.17133 \\
.17788 \\
.18433 \\
.19092 \\
.19762\end{array}$ \\
\hline $\begin{array}{l}300 . \\
310 . \\
320 . \\
330 . \\
340 . \\
350 . \\
360 . \\
370 . \\
380 . \\
390 .\end{array}$ & $\begin{array}{l}.1499 \\
.1450 \\
.1404 \\
.1361 \\
.1320 \\
.1282 \\
.1246 \\
.1212 \\
.1180 \\
.1150\end{array}$ & $\begin{array}{l}.00899 \\
.00927 \\
.00956 \\
.00984 \\
.01012 \\
.01039 \\
.01066 \\
.011093 \\
.01120 \\
.01146\end{array}$ & $\begin{array}{r}8.32 \\
8.58 \\
8.84 \\
9.09 \\
9.35 \\
9.59 \\
9.84 \\
10.08 \\
10.32 \\
10.56\end{array}$ & $\begin{array}{l}.27342 \\
.29190 \\
.31087 \\
.33030 \\
.35019 \\
.37052 \\
.39129 \\
.41250 \\
.43413 \\
.45619\end{array}$ & $\begin{array}{l}300 . \\
310 . \\
320 . \\
330 . \\
340 . \\
350 . \\
360 . \\
370 . \\
380 . \\
390 .\end{array}$ & $\begin{array}{l}.2003 \\
.1937 \\
.1875 \\
.1817 \\
.1763 \\
.1712 \\
.1664 \\
.1618 \\
.1575 \\
.1534\end{array}$ & $\begin{array}{l}.00900 \\
.00929 \\
.00957 \\
.00985 \\
.01013 \\
.01040 \\
.01067 \\
.01094 \\
.01121 \\
.01147\end{array}$ & $\begin{array}{r}8.33 \\
8.59 \\
8.85 \\
9.10 \\
9.35 \\
9.60 \\
9.84 \\
10.09 \\
10.33 \\
10.57\end{array}$ & $\begin{array}{l}.20442 \\
.21832 \\
.23258 \\
.24710 \\
.26213 \\
.27740 \\
.29301 \\
.30894 \\
.32520 \\
.34177\end{array}$ \\
\hline $\begin{array}{l}400 . \\
410 . \\
420 . \\
430 . \\
440 . \\
450 . \\
460 . \\
470 . \\
480 . \\
490 .\end{array}$ & $\begin{array}{l}.1121 \\
.1093 \\
.1067 \\
.1042 \\
.1018 \\
.0995 \\
.0974 \\
.0953 \\
.0933 \\
.0914\end{array}$ & $\begin{array}{l}.01172 \\
.01198 \\
.01224 \\
.011250 \\
.011275 \\
.011300 \\
.01325 \\
.011350 \\
.011375 \\
.01400\end{array}$ & $\begin{array}{l}10.80 \\
11.03 \\
11.26 \\
11.49 \\
11.72 \\
11.94 \\
12.16 \\
12.38 \\
12.60 \\
12.81\end{array}$ & $\begin{array}{l}.47867 \\
.50157 \\
.52488 \\
.54859 \\
.57271 \\
.59722 \\
.62213 \\
.64743 \\
.67311 \\
.69917\end{array}$ & $\begin{array}{l}400 . \\
410 . \\
420 . \\
430 . \\
440 . \\
450 . \\
460 . \\
470 . \\
480 . \\
490 .\end{array}$ & $\begin{array}{l}.1495 \\
.1458 \\
.1423 \\
.1390 \\
.1358 \\
.1328 \\
.1299 \\
.1271 \\
.1244 \\
.1219\end{array}$ & $\begin{array}{l}.01173 \\
.01199 \\
.01225 \\
.01251 \\
.01276 \\
.01301 \\
.01326 \\
.01351 \\
.01376 \\
.01401\end{array}$ & $\begin{array}{l}10.80 \\
11.03 \\
11.26 \\
11.49 \\
11.72 \\
11.94 \\
12.16 \\
12.38 \\
12.60 \\
12.82\end{array}$ & $\begin{array}{l}.35865 \\
.37585 \\
.39335 \\
.41116 \\
.42927 \\
.44767 \\
.46638 \\
.48537 \\
.50465 \\
.52422\end{array}$ \\
\hline $\begin{array}{l}500 . \\
510 . \\
520 . \\
530 . \\
540 .\end{array}$ & $\begin{array}{l}.0895 \\
.0878 \\
.0861 \\
.0845 \\
.0829\end{array}$ & $\begin{array}{l}.01424 \\
.01449 \\
.01473 \\
.01497 \\
.01522\end{array}$ & $\begin{array}{l}13.03 \\
13.24 \\
13.45 \\
13.66 \\
13.86\end{array}$ & $\begin{array}{r}.72560 \\
.75240 \\
.77956 \\
.80708 \\
.83494\end{array}$ & $\begin{array}{l}500 . \\
510 . \\
520 . \\
530 . \\
540 .\end{array}$ & $\begin{array}{l}.1194 \\
.1171 \\
.1148 \\
.1126 \\
.1105\end{array}$ & $\begin{array}{l}.01425 \\
.01450 \\
.01474 \\
.01498 \\
.01523\end{array}$ & $\begin{array}{l}13.03 \\
13.24 \\
13.45 \\
13.66 \\
13.86\end{array}$ & $\begin{array}{l}.54406 \\
.56418 \\
.58457 \\
.60523 \\
.62614\end{array}$ \\
\hline
\end{tabular}


Table 7. Transoart Properties oe Dxyoen. Isotars, Engr. Units.

25. Dsia Isobar

30. osia Isobat

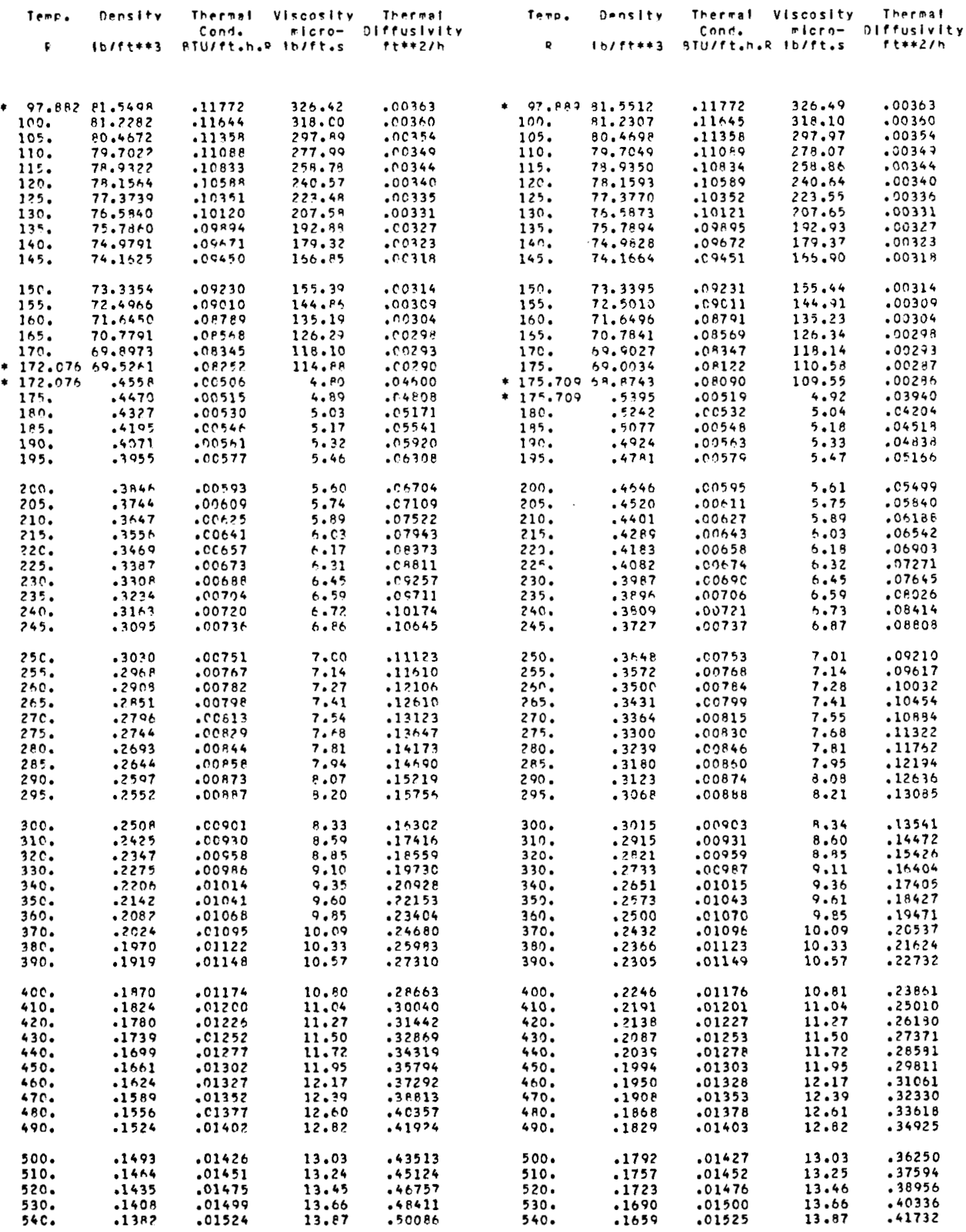

* Two phase Boundary 
Table 7. Transport Prodertles of Oxygen. Isobars, Engr. Units.

35. pst. Isober

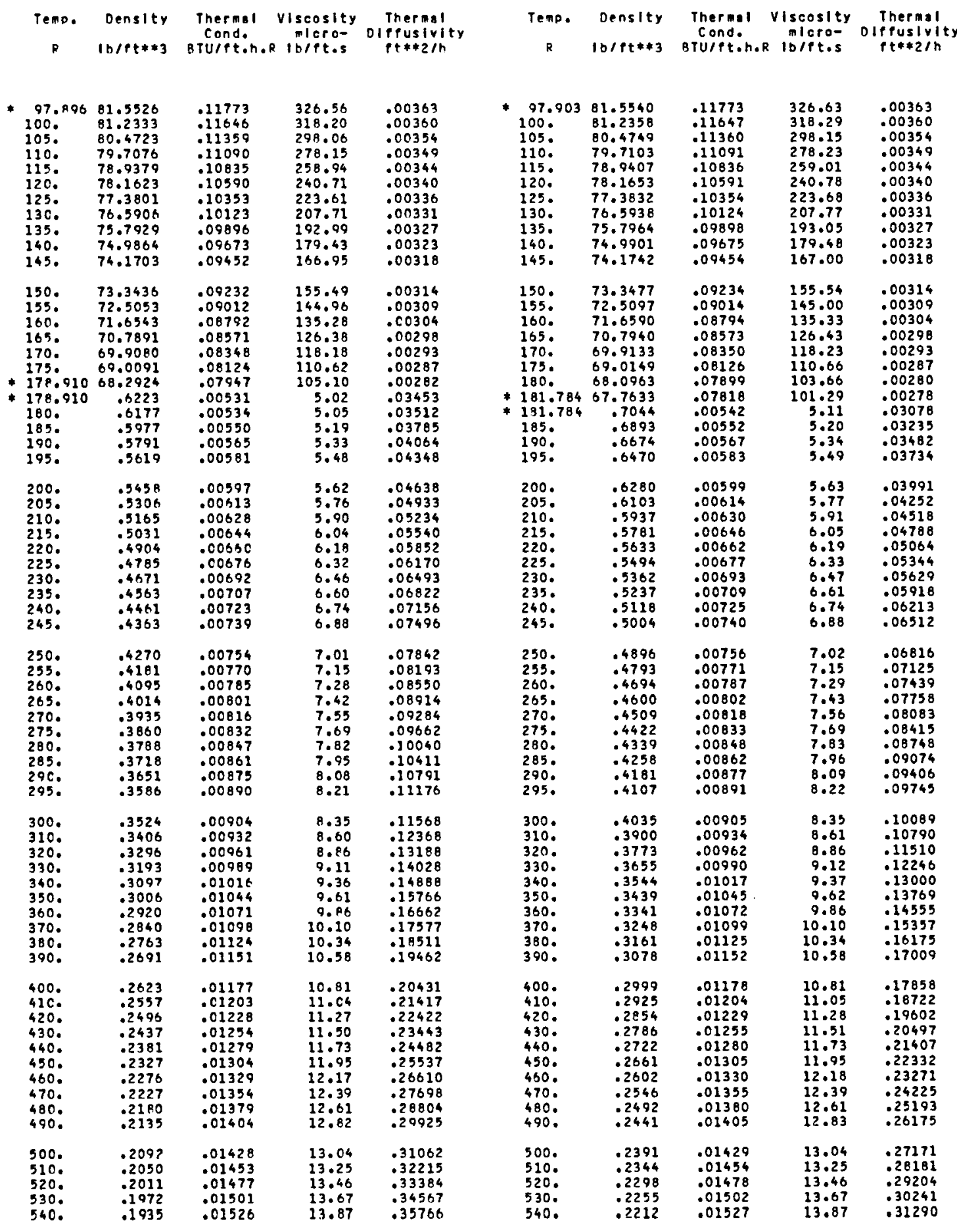


Table 7. Transport Propertles of Oxygen, Isobars, Engr. Unlts.

45. pslo Isober

50. Dsi. Isobar

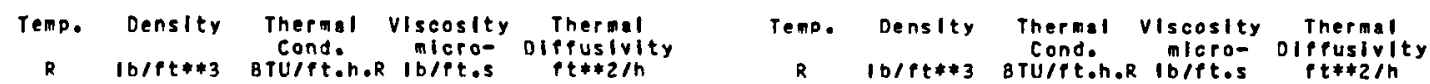
$t \neq \notin 2, h$

\begin{tabular}{|c|c|c|c|c|}
\hline $\begin{array}{l}91.918 \\
100 . \\
105 . \\
110 . \\
115 . \\
120 . \\
125 . \\
1300^{\circ} \\
135 . \\
140 . \\
145 .\end{array}$ & $\begin{array}{l}81.5567 \\
81.2408 \\
80.4801 \\
79.7156 \\
78.9463 \\
78.1712 \\
77.3894 \\
76.6004 \\
75.8033 \\
74.9974 \\
74.1819\end{array}$ & $\begin{array}{l}.11774 \\
.11649 \\
.11362 \\
.11093 \\
.10838 \\
.10593 \\
.10356 \\
.10126 \\
.09900 \\
.09677 \\
.09456\end{array}$ & $\begin{array}{l}326.78 \\
318.49 \\
298.33 \\
278.40 \\
259.16 \\
240.92 \\
223.81 \\
207.90 \\
193.17 \\
179.60 \\
167.11\end{array}$ & $\begin{array}{l}.00363 \\
.00360 \\
.00354 \\
.00349 \\
.00344 \\
.00340 \\
.00336 \\
.00331 \\
.00327 \\
.00323 \\
.00318\end{array}$ \\
\hline $\begin{array}{l}150 . \\
155 . \\
160 . \\
165 . \\
170 . \\
175 . \\
180 . \\
185 . \\
186.808 \\
185.808 \\
190 . \\
195 .\end{array}$ & $\begin{array}{r}73.3559 \\
72.5185 \\
71.6683 \\
70.8040 \\
69.9240 \\
69.0263 \\
68.1087 \\
67.1683 \\
66.8221 \\
.8674 \\
.8488 \\
.8216\end{array}$ & $\begin{array}{l}.09236 \\
.09017 \\
.08797 \\
.08576 \\
.08353 \\
.08129 \\
.07903 \\
.07674 \\
.07591 \\
.00562 \\
.00571 \\
.00587\end{array}$ & $\begin{array}{r}155.64 \\
145.10 \\
135.42 \\
126.51 \\
118.31 \\
110.74 \\
103.74 \\
97.25 \\
95.02 \\
5.27 \\
5.36 \\
5.50\end{array}$ & $\begin{array}{l}.00314 \\
.00309 \\
.00304 \\
.00299 \\
.00293 \\
.00287 \\
.00281 \\
.00274 \\
.00271 \\
.02534 \\
.02665 \\
.02872\end{array}$ \\
\hline $\begin{array}{l}200 . \\
205 . \\
210 . \\
215 . \\
220 . \\
225 . \\
230 . \\
235 . \\
240 . \\
245 .\end{array}$ & $\begin{array}{l}.7964 \\
.7730 \\
.7511 \\
.7306 \\
.7114 \\
.6933 \\
.6762 \\
.6600 \\
.6446 \\
.6300\end{array}$ & $\begin{array}{l}.00602 \\
.00618 \\
.00634 \\
.00649 \\
.00665 \\
.00681 \\
.00698 \\
.00712 \\
.00728 \\
.00743\end{array}$ & $\begin{array}{l}5.64 \\
5.79 \\
5.93 \\
6.07 \\
6.21 \\
6.34 \\
6.48 \\
6.62 \\
6.76 \\
6.90\end{array}$ & $\begin{array}{l}.03083 \\
.03296 \\
.03513 \\
.03734 \\
.03958 \\
.04186 \\
.04417 \\
.04652 \\
.04890 \\
.05132\end{array}$ \\
\hline $\begin{array}{l}250 . \\
255 . \\
260 . \\
265 . \\
270 . \\
275 . \\
280 . \\
285 . \\
290 . \\
295 .\end{array}$ & $\begin{array}{l}.6162 \\
.6029 \\
.5903 \\
.5782 \\
.5666 \\
.5555 \\
.5449 \\
.5347 \\
.5249 \\
.5154\end{array}$ & $\begin{array}{l}.00759 \\
.00774 \\
.00790 \\
.00805 \\
.00820 \\
.00838 \\
.00851 \\
.00865 \\
.00879 \\
.00893\end{array}$ & $\begin{array}{l}7.03 \\
7.17 \\
7.30 \\
7.44 \\
7.57 \\
7.70 \\
7.84 \\
7.97 \\
8.10 \\
6.23\end{array}$ & $\begin{array}{l}.05370 \\
.05628 \\
.05882 \\
.06140 \\
.06402 \\
.06670 \\
.06939 \\
.07200 \\
.07468 \\
.07740\end{array}$ \\
\hline $\begin{array}{l}300 . \\
310 . \\
320 . \\
330 . \\
340 . \\
350 . \\
360 . \\
370 . \\
380 . \\
390 .\end{array}$ & $\begin{array}{l}.5063 \\
.4891 \\
.4731 \\
.4581 \\
.4441 \\
.4309 \\
.4185 \\
.4068 \\
.3958 \\
.3854\end{array}$ & $\begin{array}{l}.00908 \\
.00936 \\
.00964 \\
.00992 \\
.01020 \\
.01047 \\
.01074 \\
.011101 \\
.01127 \\
.01154\end{array}$ & $\begin{array}{r}8.36 \\
8.62 \\
8.87 \\
9.13 \\
9.38 \\
9.62 \\
9.87 \\
10.11 \\
10.35 \\
10.59\end{array}$ & $\begin{array}{l}.08017 \\
.08381 \\
.09159 \\
.09751 \\
.10356 \\
.10974 \\
.11605 \\
.12249 \\
.12905 \\
.13574\end{array}$ \\
\hline $\begin{array}{l}400 . \\
410 . \\
420 . \\
430 . \\
440 . \\
450 . \\
460 . \\
470 . \\
480 . \\
490 .\end{array}$ & $\begin{array}{l}.3755 \\
.3661 \\
.3572 \\
.3487 \\
.3406 \\
.3329 \\
.3255 \\
.3185 \\
.3117 \\
.3053\end{array}$ & $\begin{array}{l}.01180 \\
.01206 \\
.01231 \\
.01257 \\
.01282 \\
.01307 \\
.01332 \\
.01357 \\
.01382 \\
.01407\end{array}$ & $\begin{array}{l}10.82 \\
11.05 \\
11.28 \\
11.51 \\
11.74 \\
11.96 \\
12.18 \\
12.40 \\
12.62 \\
12.83\end{array}$ & $\begin{array}{r}.14256 \\
.14949 \\
.15655 \\
.16372 \\
.17102 \\
.17844 \\
.18597 \\
.19361 \\
.20138 \\
.20925\end{array}$ \\
\hline $\begin{array}{l}500 . \\
510 . \\
520 . \\
530 . \\
540 .\end{array}$ & $\begin{array}{l}.2991 \\
.2931 \\
.2874 \\
.2819 \\
.2767\end{array}$ & $\begin{array}{l}.01431 \\
.01456 \\
.01480 \\
.01504 \\
.01529\end{array}$ & $\begin{array}{l}13.05 \\
13.26 \\
13.47 \\
13.67 \\
13.88\end{array}$ & $\begin{array}{l}.21723 \\
.22533 \\
.23353 \\
.24183 \\
.25024\end{array}$ \\
\hline
\end{tabular}


Toble 7. Tronsoopt Propeftles of Oxyoen, Isobars, Engr. Units.

100. Psia Isobar

150. DSi. Isobar

\begin{tabular}{|c|c|c|c|c|c|c|c|c|c|}
\hline Temp. & $\begin{array}{l}\text { Density } \\
10 / f t * * 3\end{array}$ & $\begin{array}{l}\text { Thermal } \\
\text { Cond. } \\
\text { BTUlftehor }\end{array}$ & $\begin{array}{c}\text { viscosity } \\
\text { mlero- } \\
\text { Ib/ftos }\end{array}$ & $\begin{array}{c}\text { Thermal } \\
\text { Diffusivity } \\
\text { ft**2/h }\end{array}$ & $\begin{array}{c}\text { Teno. } \\
\text { R }\end{array}$ & $\begin{array}{l}\text { Denslty } \\
I b / f t \neq \neq 3\end{array}$ & $\begin{array}{l}\text { Thernal } \\
\text { Cond. } \\
\text { B TUlftin.R }\end{array}$ & $\begin{array}{l}\text { Viscosity } \\
\text { micro- } \\
\text { Ib/ftes }\end{array}$ & $\begin{array}{l}\text { Thermil } \\
\text { oIffusivit } \\
\text { ft } * 2 / h\end{array}$ \\
\hline $\begin{array}{l}97.989 \\
100 . \\
1050 \\
110 . \\
1150 \\
120 . \\
1250 \\
130 . \\
1350 \\
140 . \\
145 .\end{array}$ & $\begin{array}{l}81.3707 \\
81.2658 \\
80.5060 \\
79.7425 \\
78.9744 \\
78.2006 \\
77.4204 \\
76.6331 \\
75.8378 \\
75.0339 \\
74.2206\end{array}$ & $\begin{array}{l}.11779 \\
.11658 \\
.11372 \\
.11103 \\
.10848 \\
.10603 \\
.10367 \\
.10137 \\
.09912 \\
.09689 \\
.09469\end{array}$ & $\begin{array}{l}327.50 \\
319.45 \\
299.22 \\
279.22 \\
259.93 \\
241.63 \\
224.48 \\
208.32 \\
193.76 \\
180.15 \\
167.64\end{array}$ & $\begin{array}{l}.00363 \\
.00361 \\
.00355 \\
.00349 \\
.00345 \\
.00340 \\
.00336 \\
.00332 \\
.00328 \\
.00323 \\
.00319\end{array}$ & $\begin{array}{l}\text { * } 98.060 \\
100 . \\
105 . \\
110 . \\
115 . \\
120 . \\
125 . \\
130 . \\
135 . \\
140 . \\
145 .\end{array}$ & $\begin{array}{l}81.5846 \\
81.2908 \\
80.5318 \\
79.7694 \\
79.0025 \\
78.2301 \\
77.6514 \\
76.6557 \\
75.8722 \\
75.0703 \\
74.2591\end{array}$ & $\begin{array}{l}.11784 \\
.11667 \\
.11381 \\
.11112 \\
.10858 \\
.10614 \\
.10378 \\
.10148 \\
.09923 \\
.09702 \\
.09482\end{array}$ & $\begin{array}{l}328.22 \\
320.42 \\
300.11 \\
280.04 \\
260.69 \\
242.34 \\
225.14 \\
209.14 \\
194.35 \\
180.71 \\
168.17\end{array}$ & $\begin{array}{l}.00363 \\
.00361 \\
.00355 \\
.00350 \\
.00345 \\
.00341 \\
.00336 \\
.00332 \\
.00328 \\
.00324 \\
.00319\end{array}$ \\
\hline $\begin{array}{l}150 . \\
155 . \\
160 . \\
165 . \\
170 . \\
175 . \\
180 . \\
185 . \\
190 . \\
195 .\end{array}$ & $\begin{array}{l}73.3970 \\
72.5621 \\
71.7147 \\
70.8536 \\
69.9770 \\
69.0832 \\
68.1699 \\
67.2345 \\
66.2739 \\
65.2642\end{array}$ & $\begin{array}{l}.09250 \\
.09031 \\
.08811 \\
.08591 \\
.08370 \\
.08146 \\
.07921 \\
.07693 \\
.07462 \\
.07229\end{array}$ & $\begin{array}{r}156.14 \\
145.58 \\
135.88 \\
126.96 \\
118.75 \\
111.17 \\
104.16 \\
97.66 \\
91.62 \\
85.99\end{array}$ & $\begin{array}{l}.00314 \\
.00310 \\
.00304 \\
.00299 \\
.00294 \\
.00288 \\
.00281 \\
.00275 \\
.00268 \\
.00260\end{array}$ & $\begin{array}{l}150 . \\
155 . \\
160 . \\
165 . \\
170 . \\
275 . \\
180 . \\
185 . \\
190 . \\
195 .\end{array}$ & $\begin{array}{l}73.4379 \\
72.6055 \\
71.7610 \\
70.9029 \\
70.0298 \\
69.1398 \\
68.2308 \\
67.3002 \\
66.3451 \\
65.3618\end{array}$ & $\begin{array}{l}.09263 \\
.09045 \\
.08826 \\
.08606 \\
.08386 \\
.08163 \\
.07939 \\
.07712 \\
.07482 \\
.07250\end{array}$ & $\begin{array}{l}156.64 \\
146.06 \\
136.35 \\
127.41 \\
119.18 \\
111.59 \\
104.57 \\
98.07 \\
92.02 \\
86.39\end{array}$ & $\begin{array}{l}.00315 \\
.00310 \\
.00305 \\
.00300 \\
.00294 \\
.00288 \\
.00282 \\
.00276 \\
.00269 \\
.00261\end{array}$ \\
\hline $\begin{array}{l}200 . \\
204.420 \\
204.420 \\
205 . \\
210 . \\
215 . \\
220 . \\
225 . \\
230 . \\
235 . \\
240 . \\
245 .\end{array}$ & $\begin{array}{l}64.2609 \\
63.3238 \\
1.6730 \\
1.6659 \\
1.6075 \\
1.5543 \\
1.5055 \\
1.4605 \\
1.4187 \\
1.3798 \\
1.3434 \\
1.3093\end{array}$ & $\begin{array}{l}.06991 \\
.06778 \\
.00636 \\
.00638 \\
.00652 \\
.00667 \\
.00683 \\
.00698 \\
.00713 \\
.00728 \\
.00743 \\
.00759\end{array}$ & $\begin{array}{l}80.71 \\
76.31 \\
5.87 \\
5.88 \\
6.02 \\
6.16 \\
6.29 \\
6.43 \\
6.57 \\
6.70 \\
6.84 \\
6.97\end{array}$ & $\begin{array}{l}.00252 \\
.00244 \\
.01351 \\
.01365 \\
.01467 \\
.01609 \\
.01732 \\
.01857 \\
.01982 \\
.02108 \\
.02236 \\
.02365\end{array}$ & $\begin{array}{l}200 . \\
205 . \\
210 . \\
215 . \\
+216.364 \\
+216.364 \\
220 . \\
225 . \\
230 . \\
235 . \\
240 . \\
245 .\end{array}$ & $\begin{array}{l}64.3459 \\
63.2921 \\
62.1936 \\
61.0419 \\
60.7172 \\
2.4860 \\
2.4174 \\
2.3293 \\
2.2499 \\
2.1775 \\
2.1111 \\
2.0498\end{array}$ & $\begin{array}{l}.07014 \\
.06774 \\
.06529 \\
.06279 \\
.06209 \\
.00694 \\
.00704 \\
.00718 \\
.00732 \\
.00747 \\
.00762 \\
.00776\end{array}$ & $\begin{array}{l}81.11 \\
76.16 \\
71.48 \\
67.04 \\
65.87 \\
6.31 \\
6.41 \\
6.54 \\
6.67 \\
6.80 \\
6.93 \\
7.07\end{array}$ & $\begin{array}{l}.00253 \\
.00244 \\
.00235 \\
.00225 \\
.00222 \\
.00905 \\
.00973 \\
.01065 \\
.01156 \\
.01246 \\
.01340 \\
.01433\end{array}$ \\
\hline $\begin{array}{l}300 . \\
310 . \\
320 . \\
330 . \\
340 . \\
350 . \\
360 . \\
370 . \\
380 . \\
390 .\end{array}$ & $\begin{array}{r}1.0326 \\
.9955 \\
.9612 \\
.9294 \\
.8997 \\
.8720 \\
.8460 \\
.8216 \\
.7986 \\
.7770\end{array}$ & $\begin{array}{l}.00921 \\
.00948 \\
.00976 \\
.01004 \\
.01031 \\
.01058 \\
.01085 \\
.01112 \\
.01138 \\
.01164\end{array}$ & $\begin{array}{r}8.42 \\
0.68 \\
8.93 \\
9.18 \\
9.43 \\
9.67 \\
9.92 \\
10.16 \\
10.39 \\
10.63\end{array}$ & $\begin{array}{l}.03869 \\
.04158 \\
.04455 \\
.04757 \\
.05056 \\
.05382 \\
.05703 \\
.06030 \\
.06364 \\
.06703\end{array}$ & $\begin{array}{l}300 . \\
310 . \\
320 . \\
330 . \\
340 . \\
350 . \\
360 . \\
370 . \\
380 . \\
390 .\end{array}$ & $\begin{array}{l}1.5808 \\
1.5207 \\
1.4656 \\
1.4147 \\
1.3675 \\
1.3237 \\
1.2828 \\
1.2446 \\
1.2087 \\
1.1750\end{array}$ & $\begin{array}{l}.00935 \\
.00962 \\
.00989 \\
.01016 \\
.01043 \\
.01070 \\
.01096 \\
.01123 \\
.011149 \\
.01175\end{array}$ & $\begin{array}{r}8.49 \\
8.74 \\
8.99 \\
9.24 \\
9.48 \\
9.73 \\
9.97 \\
10.21 \\
10.44 \\
10.68\end{array}$ & $\begin{array}{l}.02484 \\
.02682 \\
.02884 \\
.03091 \\
.03301 \\
.03516 \\
.03734 \\
.03956 \\
.04182 \\
.04412\end{array}$ \\
\hline $\begin{array}{l}400 . \\
410 . \\
420 . \\
430 . \\
440 . \\
450 . \\
460 . \\
470 . \\
480 . \\
490 .\end{array}$ & $\begin{array}{l}.7565 \\
.7371 \\
.7188 \\
.7013 \\
.6847 \\
.6689 \\
.6339 \\
.6395 \\
.6257 \\
.6126\end{array}$ & $\begin{array}{l}.01190 \\
.01216 \\
.01242 \\
.01267 \\
.01292 \\
.01317 \\
.01342 \\
.01367 \\
.01392 \\
.01417\end{array}$ & $\begin{array}{l}10.86 \\
11.09 \\
11.32 \\
11.53 \\
11.77 \\
11.99 \\
12.21 \\
12.43 \\
12.65 \\
12.86\end{array}$ & $\begin{array}{l}.07049 \\
.07401 \\
.07758 \\
.08121 \\
.08491 \\
.08666 \\
.09246 \\
.09633 \\
.10025 \\
.10422\end{array}$ & $\begin{array}{l}400 . \\
410 . \\
420 . \\
430 . \\
440 . \\
450 . \\
460 . \\
470 . \\
480 . \\
490 .\end{array}$ & $\begin{array}{r}1.1432 \\
1.1132 \\
1.0849 \\
1.0580 \\
1.0324 \\
1.0082 \\
.9850 \\
.9630 \\
.9420 \\
.9219\end{array}$ & $\begin{array}{l}.01201 \\
.01226 \\
.01152 \\
.01277 \\
.01302 \\
.01327 \\
.01352 \\
.011377 \\
.01402 \\
.01426\end{array}$ & $\begin{array}{l}10.91 \\
11.14 \\
11.36 \\
11.59 \\
11.81 \\
12.03 \\
12.25 \\
12.47 \\
12.68 \\
12.90\end{array}$ & $\begin{array}{l}.04646 \\
.04884 \\
.05125 \\
.05370 \\
.05629 \\
.05872 \\
.08129 \\
.05389 \\
.06659 \\
.06921\end{array}$ \\
\hline $\begin{array}{l}500 . \\
510 . \\
520 . \\
530 . \\
540 .\end{array}$ & $\begin{array}{l}.6000 \\
.5879 \\
.5763 \\
.5652 \\
.5545\end{array}$ & $\begin{array}{l}.01441 \\
.01465 \\
.01490 \\
.01514 \\
.01538\end{array}$ & $\begin{array}{l}13.07 \\
13.28 \\
13.49 \\
13.70 \\
13.90\end{array}$ & $\begin{array}{r}.10825 \\
.11234 \\
.11647 \\
.12066 \\
.12490\end{array}$ & $\begin{array}{l}500 . \\
510 . \\
520 . \\
530 . \\
540 .\end{array}$ & $\begin{array}{l}.9027 \\
.8842 \\
.8666 \\
.8496 \\
.8334\end{array}$ & $\begin{array}{l}.01451 \\
.01475 \\
.01499 \\
.01523 \\
.01547\end{array}$ & $\begin{array}{l}13.11 \\
13.32 \\
13.52 \\
13.73 \\
13.93\end{array}$ & $\begin{array}{l}.07192 \\
.07467 \\
.07745 \\
.08026 \\
.08311\end{array}$ \\
\hline
\end{tabular}


Table 7. Transoort Propertles of Aryoen, isobars, Enor. Unlts.

200. nsi. Isotigr

250. Dsla Isobar

\begin{tabular}{|c|c|c|c|c|c|c|c|c|c|}
\hline $\begin{array}{c}\text { Teno. } \\
p\end{array}$ & $\begin{array}{l}\text { Pensity } \\
\ln /+t * 3\end{array}$ & $\begin{array}{l}\text { Thermat } \\
\text { Cond. } \\
\text { BTU/ft.h.R }\end{array}$ & $\begin{array}{c}\text { viscosity } \\
\text { micro- } \\
\text { inftios }\end{array}$ & $\begin{array}{c}\text { Thertal } \\
\text { Diffusivite } \\
\text { ft**2at }\end{array}$ & Teno. & $\begin{array}{l}\text { Density } \\
\text { In/ft**3 }\end{array}$ & $\begin{array}{l}\text { Thertal } \\
\text { Cond. } \\
\text { RTu/ftan.e }\end{array}$ & $\begin{array}{c}v i s \cos i t y \\
\text { rlero- } \\
\text { Ib/ftes }\end{array}$ & $\begin{array}{c}\text { Thefinal } \\
\text { Diffusivity } \\
\text { ft*zidh }\end{array}$ \\
\hline $\begin{array}{l}95.131 \\
100 . \\
105 . \\
110 . \\
115 . \\
120 . \\
175 . \\
130 . \\
1350 \\
140 . \\
145 .\end{array}$ & $\begin{array}{l}P 1.5965 \\
81.3157 \\
80.5575 \\
79.7942 \\
79.0364 \\
79.2594 \\
77.4972 \\
76.0992 \\
75.9045 \\
75.1065 \\
74.9975\end{array}$ & $\begin{array}{l}.11708 \\
.11674 \\
.11390 \\
.11192 \\
.118858 \\
.10674 \\
.10399 \\
.10140 \\
.09935 \\
.09714 \\
.09494\end{array}$ & $\begin{array}{l}329.94 \\
321.39 \\
301.00 \\
380.86 \\
261.45 \\
243.05 \\
225.90 \\
207.77 \\
194.93 \\
181.26 \\
169.00\end{array}$ & $\begin{array}{l}.00344 \\
.00331 \\
.00355 \\
.00350 \\
.00345 \\
.00341 \\
.00337 \\
.00333 \\
.00328 \\
.00324 \\
.00320\end{array}$ & $\begin{array}{l}\text { * } 99.20 ? \\
100 . \\
105 . \\
110 . \\
115 . \\
1>0 . \\
125 . \\
139 . \\
135 . \\
140 . \\
145 .\end{array}$ & $\begin{array}{l}81.6124 \\
91.3405 \\
80.5832 \\
79.8220 \\
70.0583 \\
79.2984 \\
77.5130 \\
75.7305 \\
75.9407 \\
75.1427 \\
74.3357\end{array}$ & $\begin{array}{l}.11793 \\
.11685 \\
.11400 \\
.11132 \\
.10878 \\
.10634 \\
.10399 \\
.10171 \\
.00947 \\
.09726 \\
.09507\end{array}$ & $\begin{array}{l}329.55 \\
322.36 \\
301.89 \\
281.68 \\
262.22 \\
243.76 \\
226.47 \\
210.39 \\
195.52 \\
181.82 \\
169.22\end{array}$ & $\begin{array}{l}.00334 \\
.00331 \\
.00355 \\
.00350 \\
.00345 \\
.00341 \\
.00337 \\
.00333 \\
.06329 \\
.00325 \\
.00320\end{array}$ \\
\hline $\begin{array}{l}150 . \\
155 . \\
160 . \\
165 . \\
170 \\
175 . \\
180 . \\
185 . \\
190 . \\
195 .\end{array}$ & $\begin{array}{l}73.4785 \\
72.6487 \\
71.8070 \\
70.9519 \\
70.0827 \\
69.1960 \\
68.2912 \\
67.3654 \\
64.4157 \\
65.4386\end{array}$ & $\begin{array}{l}.09276 \\
.09059 \\
.08941 \\
.08522 \\
.04402 \\
.08190 \\
.07957 \\
.07731 \\
.07502 \\
.07271\end{array}$ & $\begin{array}{l}157.15 \\
146.55 \\
135.81 \\
127.05 \\
119.01 \\
112.01 \\
104.92 \\
99.47 \\
92.42 \\
85.78\end{array}$ & $\begin{array}{l}.00315 \\
.00311 \\
.06308 \\
.00301 \\
.00295 \\
.00299 \\
.00283 \\
.00277 \\
.00270 \\
.00252\end{array}$ & $\begin{array}{l}150 . \\
155 . \\
160 . \\
155 . \\
170 . \\
175 . \\
180 . \\
185 . \\
190 . \\
195 .\end{array}$ & $\begin{array}{l}73.5191 \\
72.6018 \\
71.0527 \\
71.0007 \\
70.1362 \\
59.2518 \\
68.3511 \\
67.4300 \\
66.4856 \\
65.5147\end{array}$ & $\begin{array}{l}.09290 \\
.09072 \\
.08855 \\
.08637 \\
.08418 \\
.08197 \\
.07974 \\
.07749 \\
.07522 \\
.07292\end{array}$ & $\begin{array}{r}157.05 \\
167.03 \\
137.27 \\
128.30 \\
120.04 \\
112.43 \\
105.39 \\
94.87 \\
92.82 \\
87.19\end{array}$ & $\begin{array}{l}.00316 \\
.00311 \\
.00304 \\
.00301 \\
.00275 \\
.00270 \\
.00214 \\
.00277 \\
.00271 \\
.00263\end{array}$ \\
\hline $\begin{array}{l}200 . \\
205 . \\
210 . \\
215 . \\
220 . \\
225 . \\
225.697 \\
225.097 \\
230 . \\
2350 \\
260 . \\
245 .\end{array}$ & $\begin{array}{l}64.4300 \\
63.3847 \\
62.2953 \\
61.1570 \\
59.9563 \\
58.3805 \\
58.4959 \\
3.3293 \\
3.2074 \\
3.0819 \\
2.9701 \\
2.8594\end{array}$ & $\begin{array}{l}.07034 \\
.06797 \\
.06554 \\
.06306 \\
.05052 \\
.05791 \\
.05754 \\
.00746 \\
.00757 \\
.00770 \\
.00784 \\
.00798\end{array}$ & $\begin{array}{l}81.51 \\
76.55 \\
71.88 \\
67.45 \\
63.23 \\
59.17 \\
58.42 \\
6.69 \\
6.80 \\
6.92 \\
7.05 \\
7.18\end{array}$ & $\begin{array}{l}.00254 \\
.00245 \\
.00237 \\
.00227 \\
.00215 \\
.00205 \\
.00203 \\
.00644 \\
.00731 \\
.00807 \\
.00883 \\
.00959\end{array}$ & $\begin{array}{l}200 . \\
205 . \\
210 . \\
215 . \\
230 . \\
225 . \\
230 . \\
+233.478 \\
233.479 \\
235 . \\
240 . \\
245 .\end{array}$ & $\begin{array}{l}36.5131 \\
63.4761 \\
52.3976 \\
51.2702 \\
60.084 ? \\
58.8260 \\
57.4811 \\
53.4800 \\
4.2086 \\
4.1454 \\
3.9584 \\
3.7962\end{array}$ & $\begin{array}{l}.07058 \\
.06321 \\
.06580 \\
.06334 \\
.06082 \\
.05824 \\
.05557 \\
.05366 \\
.00797 \\
.00800 \\
.00812 \\
.00824\end{array}$ & $\begin{array}{l}91.00 \\
75.95 \\
72.29 \\
57.87 \\
63.60 \\
59.52 \\
55.71 \\
53.05 \\
7.05 \\
7.08 \\
7.20 \\
7.31\end{array}$ & $\begin{array}{l}.00255 \\
.00247 \\
.06238 \\
.00229 \\
.00218 \\
.00207 \\
.00195 \\
.00185 \\
.00510 \\
.00531 \\
.00600 \\
.00667\end{array}$ \\
\hline $\begin{array}{l}250 . \\
255 . \\
250 \\
265 . \\
270 \\
275 \\
280 . \\
285 . \\
290 . \\
295 .\end{array}$ & $\begin{array}{l}2.7778 \\
2.6939 \\
2.6165 \\
2.5448 \\
2.4780 \\
2.4155 \\
2.3569 \\
2.3016 \\
2.2495 \\
2.2002\end{array}$ & $\begin{array}{l}.00812 \\
.00824 \\
.00841 \\
.00856 \\
.00872 \\
.00888 \\
.00903 \\
.00913 \\
.00925 \\
.00937\end{array}$ & $\begin{array}{l}7.30 \\
7.43 \\
7.56 \\
7.69 \\
7.81 \\
7.04 \\
8.07 \\
8.19 \\
8.32 \\
8.44\end{array}$ & $\begin{array}{l}.01035 \\
.01110 \\
.01195 \\
.01263 \\
.01341 \\
.01620 \\
.01497 \\
.01568 \\
.01640 \\
.01714\end{array}$ & $\begin{array}{l}250 . \\
255 . \\
260 . \\
265 . \\
270 . \\
275 . \\
280 . \\
285 . \\
290 . \\
295 .\end{array}$ & $\begin{array}{l}3.6532 \\
3.5253 \\
3.4098 \\
3.3044 \\
3.2076 \\
3.1182 \\
3.0352 \\
2.9577 \\
2.8952 \\
3.8171\end{array}$ & $\begin{array}{l}.00837 \\
.00851 \\
.00865 \\
.00879 \\
.00895 \\
.00912 \\
.00926 \\
.00934 \\
.00944 \\
.00955\end{array}$ & $\begin{array}{l}7.43 \\
7.55 \\
7.58 \\
7.80 \\
7.92 \\
8.04 \\
8.17 \\
8.29 \\
8.41 \\
8.53\end{array}$ & $\begin{array}{l}.00734 \\
.00799 \\
.00855 \\
.00931 \\
.00998 \\
.01036 \\
.01132 \\
.01189 \\
.01240 \\
.011310\end{array}$ \\
\hline $\begin{array}{l}300 . \\
310 . \\
320 . \\
330 . \\
340 . \\
350 . \\
350 . \\
370 . \\
380 . \\
390 .\end{array}$ & $\begin{array}{l}2.1533 \\
2.0665 \\
1.9974 \\
1.9150 \\
1.8483 \\
1.7867 \\
1.7295 \\
1.6747 \\
1.6264 \\
1.5797\end{array}$ & $\begin{array}{l}.0 n 95 C \\
.00975 \\
.01002 \\
.01029 \\
.011055 \\
.01092 \\
.01108 \\
.01134 \\
.0116 C \\
.011186\end{array}$ & $\begin{array}{r}8.57 \\
8.82 \\
9.04 \\
9.31 \\
9.55 \\
9.79 \\
10.03 \\
10.26 \\
10.50 \\
10.73\end{array}$ & $\begin{array}{l}.01789 \\
.01942 \\
.02008 \\
.02257 \\
.02418 \\
.02582 \\
.02749 \\
.02919 \\
.03091 \\
.03268\end{array}$ & $\begin{array}{l}300 . \\
310 . \\
320 . \\
330 . \\
340 . \\
350 . \\
360 . \\
370 . \\
380 . \\
390 .\end{array}$ & $\begin{array}{l}2.7529 \\
2.6346 \\
2.5281 \\
2.4313 \\
2.3428 \\
2.2515 \\
2.1863 \\
2.1166 \\
2.0518 \\
1.9912\end{array}$ & $\begin{array}{l}.00967 \\
.00992 \\
.01017 \\
.01043 \\
.01089 \\
.01095 \\
.011121 \\
.011146 \\
.011172 \\
.011197\end{array}$ & $\begin{array}{r}8.56 \\
8.90 \\
9.14 \\
9.38 \\
9.62 \\
9.85 \\
10.09 \\
10.32 \\
10.56 \\
10.78\end{array}$ & $\begin{array}{l}.01372 \\
.01493 \\
.01628 \\
.01758 \\
.01889 \\
.02022 \\
.02158 \\
.02298 \\
.02436 \\
.02579\end{array}$ \\
\hline $\begin{array}{l}400 . \\
410 \\
420 \\
430 \\
440 \\
450 \\
460 \\
470 \\
480 \\
490 .\end{array}$ & $\begin{array}{l}1.5358 \\
1.4945 \\
1.4555 \\
1.4187 \\
1.3837 \\
1.3508 \\
1.3191 \\
1.2891 \\
1.2605 \\
1.2332\end{array}$ & $\begin{array}{l}.01212 \\
.01237 \\
.01262 \\
.01288 \\
.01313 \\
.01338 \\
.01362 \\
.01387 \\
.01412 \\
.01436\end{array}$ & $\begin{array}{l}10.96 \\
11.19 \\
11.41 \\
11.63 \\
11.86 \\
12.57 \\
12.29 \\
12.51 \\
12.72 \\
12.93\end{array}$ & $\begin{array}{l}.03444 \\
.03625 \\
.03808 \\
.03995 \\
.04184 \\
.04376 \\
.04570 \\
.04767 \\
.04967 \\
.05170\end{array}$ & $\begin{array}{l}400 . \\
410 . \\
420 . \\
430 . \\
440 . \\
450 . \\
460 . \\
470 . \\
430 . \\
490 .\end{array}$ & $\begin{array}{l}1.9344 \\
1.8811 \\
1.8309 \\
1.7835 \\
1.7387 \\
1.6963 \\
1.6560 \\
1.6177 \\
1.5813 \\
1.5465\end{array}$ & $\begin{array}{l}.01223 \\
.01248 \\
.01273 \\
.01298 \\
.011323 \\
.01348 \\
.01373 \\
.01397 \\
.01422 \\
.01446\end{array}$ & $\begin{array}{l}11.01 \\
11.24 \\
11.46 \\
11.68 \\
11.90 \\
12.12 \\
12.34 \\
12.55 \\
12.76 \\
12.97\end{array}$ & $\begin{array}{l}.02723 \\
.02870 \\
.03029 \\
.03169 \\
.03322 \\
.03478 \\
.03635 \\
.03794 \\
.03956 \\
.04120\end{array}$ \\
\hline $\begin{array}{l}500 . \\
510 . \\
520 . \\
530 \\
540 .\end{array}$ & $\begin{array}{l}1.2071 \\
1.1822 \\
1.1583 \\
1.1354 \\
1.1134\end{array}$ & $\begin{array}{l}.01460 \\
.01495 \\
.01509 \\
.01533 \\
.01557\end{array}$ & $\begin{array}{l}13.14 \\
13.35 \\
13.56 \\
13.75 \\
13.97\end{array}$ & $\begin{array}{l}.05375 \\
.05583 \\
.05793 \\
.05006 \\
.06222\end{array}$ & $\begin{array}{l}500 . \\
510 . \\
520 . \\
530 . \\
540 .\end{array}$ & $\begin{array}{l}1.5134 \\
1.4817 \\
1.4514 \\
1.4224 \\
1.3945\end{array}$ & $\begin{array}{l}.01470 \\
.01494 \\
.01518 \\
.01542 \\
.01566\end{array}$ & $\begin{array}{l}13.18 \\
13.39 \\
13.59 \\
13.80 \\
14.00\end{array}$ & $\begin{array}{l}.04285 \\
.04453 \\
.04623 \\
.04794 \\
.04968\end{array}$ \\
\hline
\end{tabular}

* Two Phase poundary 
Table 7. Transport Propertles of Oxvgen, Isobars, Engr. Units.

300. Psla Isohar

350. psla I sobar

\begin{tabular}{|c|c|c|c|c|c|c|c|c|c|}
\hline $\begin{array}{c}\text { Tenp. } \\
8\end{array}$ & $\begin{array}{l}\text { Denslty } \\
10 / f+* * 3\end{array}$ & $\begin{array}{l}\text { Thermal } \\
\text { Cond. } \\
\text { Butert.h.R }\end{array}$ & $\begin{array}{c}\text { viscosity } \\
\text { micro- } \\
\text { irlet.s }\end{array}$ & $\begin{array}{c}\text { Thermal } \\
\text { Diffusivity } \\
\text { ft**2/h }\end{array}$ & Temo. & $\begin{array}{l}\text { Density } \\
\text { Ib/tt**3 }\end{array}$ & $\begin{array}{l}\text { Thermal } \\
\text { Cond. } \\
\text { B TUlft.h.R }\end{array}$ & $\begin{array}{c}\text { viscosity } \\
\text { micro- } \\
\text { lofftes }\end{array}$ & $\begin{array}{c}\text { Inermal } \\
\text { olffusivit } \\
\text { ft* } * 2 \text { in }\end{array}$ \\
\hline $\begin{array}{l}98.274 \\
100 . \\
105 . \\
110 . \\
115 . \\
120 . \\
125 . \\
130 . \\
135 . \\
140 . \\
145 .\end{array}$ & $\begin{array}{l}81.6263 \\
81.3654 \\
80.6089 \\
79.8495 \\
79.0862 \\
78.3178 \\
77.5436 \\
76.7628 \\
75.9747 \\
75.1786 \\
74.3738\end{array}$ & $\begin{array}{l}.11798 \\
.11695 \\
.11409 \\
.11141 \\
.10897 \\
.10645 \\
.10410 \\
.10182 \\
.09958 \\
.09738 \\
.09520\end{array}$ & $\begin{array}{l}330.37 \\
323.33 \\
302.78 \\
282.51 \\
262.98 \\
244.47 \\
227.13 \\
211.01 \\
196.11 \\
192.38 \\
169.75\end{array}$ & $\begin{array}{l}.00364 \\
.00362 \\
.00356 \\
.00351 \\
.00346 \\
.00342 \\
.00337 \\
.00333 \\
.00329 \\
.00325 \\
.00327\end{array}$ & $\begin{array}{l}* 98.345 \\
100 . \\
105 . \\
110 . \\
115 . \\
120 . \\
125 . \\
130 . \\
135 . \\
140 . \\
145 .\end{array}$ & $\begin{array}{l}81.6401 \\
81.3902 \\
90.5345 \\
79.8761 \\
70.1140 \\
78.3469 \\
77.5742 \\
76.7950 \\
76.0087 \\
75.2145 \\
74.4118\end{array}$ & $\begin{array}{l}.11803 \\
.11704 \\
.11418 \\
.11151 \\
.10897 \\
.10655 \\
.10421 \\
.10193 \\
.09970 \\
.09750 \\
.09532\end{array}$ & $\begin{array}{l}331.09 \\
324.31 \\
303.68 \\
283.33 \\
263.74 \\
245.19 \\
227.90 \\
211.64 \\
195.70 \\
192.93 \\
170.29\end{array}$ & $\begin{array}{l}.00364 \\
.00362 \\
.00356 \\
.00351 \\
.00346 \\
.00342 \\
.00338 \\
.00334 \\
.00330 \\
.00325 \\
.00371\end{array}$ \\
\hline $\begin{array}{l}150 . \\
155 . \\
160 . \\
165 . \\
170 . \\
175 . \\
180 . \\
185 . \\
190 . \\
195 .\end{array}$ & $\begin{array}{l}73.5504 \\
72.7346 \\
71.8983 \\
71.0493 \\
70.1841 \\
69.3072 \\
68.4107 \\
67.4941 \\
66.5550 \\
65.5900\end{array}$ & $\begin{array}{l}.09303 \\
.09086 \\
.08870 \\
.08552 \\
.08434 \\
.08214 \\
.07992 \\
.07768 \\
.07541 \\
.07312 .\end{array}$ & $\begin{array}{l}159.15 \\
147.51 \\
137.73 \\
17.8 .74 \\
120.47 \\
112.84 \\
105.80 \\
99.27 \\
93.21 \\
87.57\end{array}$ & $\begin{array}{l}.00316 \\
.00312 \\
.00307 \\
.00302 \\
.00296 \\
.00291 \\
.00285 \\
.00278 \\
.00271 \\
.00264\end{array}$ & $\begin{array}{l}150 . \\
155 . \\
150 . \\
155 . \\
170 . \\
175 . \\
180 . \\
185 . \\
190 . \\
195 .\end{array}$ & $\begin{array}{l}73.5994 \\
72.7773 \\
71.9436 \\
71.0975 \\
70.2376 \\
69.3624 \\
68.4690 \\
67.5578 \\
56.6237 \\
65.6645\end{array}$ & $\begin{array}{l}.09316 \\
.09100 \\
.08984 \\
.08667 \\
.08450 \\
.08230 \\
.03009 \\
.07786 \\
.07561 \\
.07333\end{array}$ & $\begin{array}{r}159.65 \\
147.99 \\
138.19 \\
129.19 \\
120.90 \\
113.25 \\
109.20 \\
99.67 \\
93.61 \\
87.96\end{array}$ & $\begin{array}{l}.00317 \\
.00312 \\
.00327 \\
.00302 \\
.00277 \\
.00271 \\
.00296 \\
.00279 \\
.00277 \\
.00265\end{array}$ \\
\hline $\begin{array}{l}200 . \\
2050 \\
210 . \\
2150 \\
220 . \\
225 . \\
230 . \\
235 . \\
240 . \\
240.211 \\
240.211 \\
245 .\end{array}$ & $\begin{array}{l}64.5053 \\
63.5643 \\
62.4974 \\
61.3915 \\
60.2095 \\
58.9509 \\
57.6468 \\
56.7178 \\
54.6405 \\
54.5797 \\
5.1368 \\
4.8799\end{array}$ & $\begin{array}{l}.07080 \\
.06845 \\
.06605 \\
.06361 \\
.06111 \\
.05858 \\
.05593 \\
.05320 \\
.05036 \\
.05024 \\
.00851 \\
.00859\end{array}$ & $\begin{array}{l}82.29 \\
77.35 \\
72.18 \\
68.27 \\
34.08 \\
30.03 \\
56.18 \\
52.39 \\
48.65 \\
48.50 \\
7.39 \\
7.49\end{array}$ & $\begin{array}{l}.00257 \\
.00248 \\
.00240 \\
.00230 \\
.00220 \\
.00200 \\
.00197 \\
.00184 \\
.00160 \\
.00169 \\
.00402 \\
.00453\end{array}$ & $\begin{array}{l}200 . \\
205 . \\
210 . \\
215 . \\
220 . \\
225 . \\
230 . \\
275 . \\
240 . \\
245 . \\
* 246.180 \\
* 246.180\end{array}$ & $\begin{array}{l}34.6766 \\
53.6555 \\
62.5958 \\
51.4910 \\
60.3326 \\
59.1098 \\
57.9081 \\
53.4075 \\
54.8724 \\
53.1743 \\
52.7390 \\
3.1261\end{array}$ & $\begin{array}{l}.07102 \\
.06868 \\
.05630 \\
.06388 \\
.06140 \\
.05887 \\
.05627 \\
.05359 \\
.05081 \\
.04788 \\
.04717 \\
.00911\end{array}$ & $\begin{array}{l}92.68 \\
77.74 \\
73.08 \\
69.68 \\
64.49 \\
60.49 \\
56.63 \\
52.88 \\
49.10 \\
45.50 \\
44.62 \\
7.74\end{array}$ & $\begin{array}{l}.0025 A \\
.00250 \\
.00241 \\
.00232 \\
.00222 \\
.00211 \\
.00200 \\
.00187 \\
.00173 \\
.00157 \\
.00153 \\
.00321\end{array}$ \\
\hline $\begin{array}{l}250 . \\
255 . \\
260 . \\
2650 \\
270 . \\
275 . \\
280 . \\
285 . \\
290 . \\
295 .\end{array}$ & $\begin{array}{l}4.6541 \\
4.4598 \\
4.2891 \\
4.1371 \\
4.0001 \\
3.8756 \\
3.7614 \\
3.6561 \\
3.5585 \\
3.4676\end{array}$ & $\begin{array}{l}.00870 \\
.00901 \\
.00994 \\
.00908 \\
.00993 \\
.00941 \\
.00554 \\
.00959 \\
.00966 \\
.00976\end{array}$ & $\begin{array}{l}7.59 \\
7.70 \\
7.82 \\
7.93 \\
9.05 \\
8.16 \\
8.78 \\
8.40 \\
8.52 \\
8.64\end{array}$ & $\begin{array}{l}.00526 \\
.00587 \\
.00647 \\
.00707 \\
.00767 \\
.00870 \\
.00887 \\
.01936 \\
.00987 \\
.01039\end{array}$ & $\begin{array}{l}250 . \\
255 . \\
250 . \\
265 . \\
270 . \\
275 . \\
280 . \\
285 . \\
290 . \\
275 .\end{array}$ & $\begin{array}{l}5.8451 \\
5.5400 \\
5.2845 \\
5.0645 \\
4.8715 \\
4.6997 \\
4.5446 \\
4.4042 \\
4.2753 \\
4.1564\end{array}$ & $\begin{array}{l}.00914 \\
.00922 \\
.00932 \\
.00745 \\
.00959 \\
.00977 \\
.00989 \\
.00989 \\
.00993 \\
.01000\end{array}$ & $\begin{array}{l}7.80 \\
7.89 \\
7.99 \\
9.09 \\
9.30 \\
9.30 \\
4.41 \\
3.52 \\
8.54 \\
9.75\end{array}$ & $\begin{array}{l}.00354 \\
.00428 \\
.00434 \\
.00543 \\
.00574 \\
.00557 \\
.00711 \\
.00754 \\
.00779 \\
.00345\end{array}$ \\
\hline $\begin{array}{l}300 . \\
310 . \\
320 . \\
330 . \\
340 . \\
350 . \\
360 . \\
370 . \\
380 . \\
390 .\end{array}$ & $\begin{array}{l}3.3825 \\
3.2275 \\
3.0892 \\
3.9548 \\
2.8518 \\
2.7487 \\
2.6538 \\
2.5643 \\
2.4051 \\
7.4006\end{array}$ & $\begin{array}{l}.00086 \\
.01009 \\
.01033 \\
.01958 \\
.01083 \\
.01108 \\
.01134 \\
.01159 \\
.01134 \\
.01209\end{array}$ & $\begin{array}{r}8.75 \\
8.99 \\
9.23 \\
9.46 \\
9.70 \\
9.93 \\
10.16 \\
10.39 \\
10.42 \\
10.85\end{array}$ & $\begin{array}{l}.01097 \\
.01200 \\
.01310 \\
.01421 \\
.01534 \\
.01648 \\
.01764 \\
.01881 \\
.02000 \\
.02121\end{array}$ & $\begin{array}{l}300 . \\
310 . \\
320 . \\
330 . \\
340 . \\
350 . \\
350 . \\
370 . \\
330 . \\
390 .\end{array}$ & $\begin{array}{l}4.0462 \\
3.8476 \\
3.5726 \\
3.5157 \\
3.3763 \\
3.2480 \\
3.1325 \\
3.0255 \\
2.9258 \\
2.0352\end{array}$ & $\begin{array}{l}.01008 \\
.01029 \\
.01051 \\
.011074 \\
.01098 \\
.01123 \\
.01147 \\
.011172 \\
.01197 \\
.011222\end{array}$ & $\begin{array}{r}8.86 \\
9.37 \\
9.37 \\
9.55 \\
9.78 \\
10.01 \\
10.74 \\
10.45 \\
10.37 \\
10.41\end{array}$ & $\begin{array}{l}.00872 \\
.00948 \\
.01085 \\
.01183 \\
.01231 \\
.01391 \\
.01483 \\
.01545 \\
.01589 \\
.01793\end{array}$ \\
\hline $\begin{array}{l}400 . \\
410 . \\
420 . \\
430 . \\
440 . \\
450 . \\
440 . \\
470 . \\
480 . \\
490 .\end{array}$ & $\begin{array}{l}2.3390 \\
2.3730 \\
2.7109 \\
? .1525 \\
? .397 ? \\
2.0457 \\
1.9959 \\
1.9489 \\
1.0043 \\
1.9319\end{array}$ & $\begin{array}{l}.01235 \\
.01250 \\
.01244 \\
.01309 \\
.01334 \\
.01359 \\
.01393 \\
.01407 \\
.01432 \\
.01456\end{array}$ & $\begin{array}{l}11.07 \\
11.29 \\
11.51 \\
11.73 \\
11.95 \\
12.17 \\
12.38 \\
12.59 \\
12.91 \\
13.01\end{array}$ & $\begin{array}{l}.02243 \\
.02367 \\
.02492 \\
.02619 \\
.02748 \\
.02979 \\
.03017 \\
.03146 \\
.03782 \\
.03414\end{array}$ & $\begin{array}{l}400 . \\
410 . \\
420 . \\
430 . \\
440 . \\
450 . \\
460 . \\
470 . \\
480 . \\
490 .\end{array}$ & $\begin{array}{l}2.7499 \\
? .5703 \\
2.5957 \\
? .5255 \\
? .4594 \\
2.3973 \\
? .3384 \\
? .2326 \\
2.2296 \\
2.1792\end{array}$ & $\begin{array}{l}.01247 \\
.01271 \\
.01296 \\
.01321 \\
.01345 \\
.01369 \\
.011394 \\
.01418 \\
.01462 \\
.01466\end{array}$ & $\begin{array}{l}11.13 \\
11.35 \\
11.57 \\
11.79 \\
13.01 \\
12.22 \\
12.43 \\
1 ? .64 \\
12.85 \\
13.06\end{array}$ & $\begin{array}{l}.01900 \\
.02007 \\
.02115 \\
.02237 \\
.02339 \\
.02452 \\
.02507 \\
.02683 \\
.02301 \\
.02920\end{array}$ \\
\hline $\begin{array}{l}500 . \\
510 . \\
520 . \\
530 . \\
540 .\end{array}$ & $\begin{array}{l}1.8714 \\
1.7878 \\
1.7459 \\
1.7106 \\
1.3767\end{array}$ & $\begin{array}{r}.01490 \\
.01504 \\
.01528 \\
.01552 \\
.01578\end{array}$ & $\begin{array}{l}13.22 \\
13.43 \\
13.03 \\
13.83 \\
14.04\end{array}$ & $\begin{array}{l}.03550 \\
.03700 \\
.03942 \\
.03996 \\
.04132\end{array}$ & $\begin{array}{l}503 . \\
510 . \\
520 . \\
530 . \\
540 .\end{array}$ & $\begin{array}{l}? .1312 \\
? .0854 \\
? .0417 \\
? .0000 \\
1.0300\end{array}$ & $\begin{array}{l}.01490 \\
.01514 \\
.01538 \\
.01562 \\
.01595\end{array}$ & $\begin{array}{l}13.27 \\
13.47 \\
13.67 \\
13.87 \\
14.07\end{array}$ & $\begin{array}{l}.03040 \\
.0316 ? \\
.03295 \\
.03410 \\
.03535\end{array}$ \\
\hline
\end{tabular}


Table 7. Transoort Properties of Oxygen. Isobars, Engr. Units.

400. psis Isotar

450. Os l. Isobar

\begin{tabular}{|c|c|c|c|c|c|c|c|c|c|}
\hline Temp. & $\begin{array}{l}\text { Density } \\
\text { Ib/ }+* * * 3\end{array}$ & $\begin{array}{l}\text { Thermal } \\
\text { Cond. } \\
\text { BTUlft.h.R }\end{array}$ & $\begin{array}{c}\text { VIscosity } \\
\text { mlero- } \\
\text { Ib/ft.s }\end{array}$ & 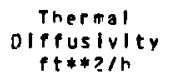 & $\begin{array}{c}\text { Teno. } \\
R\end{array}$ & 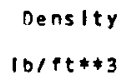 & $\begin{array}{l}\text { Thermal } \\
\text { Cond. } \\
\text { BTU/ft, }\end{array}$ & $\begin{array}{l}\text { viscosity } \\
\text { micro- } \\
\text { lo/ftos }\end{array}$ & $\begin{array}{r}\text { Thermsi } \\
01 f f u s i v i \\
\text { ft*21h }\end{array}$ \\
\hline $\begin{array}{l}98.416 \\
100 . \\
105 . \\
110 . \\
1150 \\
120 . \\
125 \\
130 . \\
1350 \\
140 \\
145 .\end{array}$ & $\begin{array}{l}81.5540 \\
81.4150 \\
80.6400 \\
79.9027 \\
79.1417 \\
78.3740 \\
77.4047 \\
74.8271 \\
75.0475 \\
75.250 ? \\
74.4495\end{array}$ & $\begin{array}{l}.11808 \\
.11713 \\
.11428 \\
.11161 \\
.10907 \\
.10645 \\
.10439 \\
.10204 \\
.09981 \\
.09742 \\
.00545\end{array}$ & $\begin{array}{l}331.80 \\
325.28 \\
304.57 \\
284.16 \\
254.51 \\
245.90 \\
228.46 \\
212.26 \\
197.28 \\
193.49 \\
170.80\end{array}$ & $\begin{array}{l}.00364 \\
.00362 \\
.00356 \\
.00351 \\
.00347 \\
.00342 \\
.00338 \\
.00334 \\
.00330 \\
.00326 \\
.00322\end{array}$ & $\begin{array}{l}98.487 \\
100 . \\
105 . \\
110 . \\
115 . \\
120 . \\
125 . \\
130 . \\
135 . \\
140 . \\
145 .\end{array}$ & $\begin{array}{l}81.6678 \\
81.4397 \\
80.6855 \\
79.9292 \\
79.1693 \\
78.4049 \\
77.6351 \\
75.8591 \\
76.0763 \\
75.2859 \\
74.4972\end{array}$ & $\begin{array}{l}.11812 \\
.11722 \\
.11437 \\
.11170 \\
.10917 \\
.10675 \\
.10442 \\
.10215 \\
.09993 \\
.09774 \\
.09558\end{array}$ & $\begin{array}{l}332.51 \\
326.26 \\
305.47 \\
284.98 \\
265.27 \\
246.61 \\
229.13 \\
212.89 \\
197.87 \\
184.04 \\
171.33\end{array}$ & $\begin{array}{l}.00364 \\
.00362 \\
.00357 \\
.00351 \\
.00347 \\
.00343 \\
.00339 \\
.00335 \\
.00330 \\
.00326 \\
.00322\end{array}$ \\
\hline $\begin{array}{l}150 . \\
155 . \\
160 . \\
135 . \\
170 . \\
175 .\end{array}$ & $\begin{array}{l}73.6397 \\
72.9197 \\
71.9897 \\
71.1455 \\
70.7899 \\
69.4171\end{array}$ & $\begin{array}{r}.09329 \\
.09114 \\
.08898 \\
.08682 \\
.08465 \\
.06 ? 47\end{array}$ & $\begin{array}{l}159.16 \\
148.46 \\
138.45 \\
129.63 \\
121.33 \\
113.58\end{array}$ & $\begin{array}{l}.00317 \\
.00313 \\
.00308 \\
.00303 \\
.00298 \\
.0029 ?\end{array}$ & $\begin{array}{l}150 . \\
155 . \\
160 . \\
155 . \\
170 . \\
175 .\end{array}$ & $\begin{array}{l}73.6795 \\
72.8620 \\
72.0336 \\
71.1933 \\
70.3398 \\
39.4715\end{array}$ & $\begin{array}{l}.09342 \\
.09128 \\
.08913 \\
.08697 \\
.08481 \\
.08263\end{array}$ & $\begin{array}{l}159.66 \\
146.94 \\
139.11 \\
130.07 \\
121.76 \\
114.09\end{array}$ & $\begin{array}{l}.00318 \\
.00313 \\
.00309 \\
.00304 \\
.00298 \\
.00293\end{array}$ \\
\hline $\begin{array}{l}190 . \\
185 \\
196 . \\
195 .\end{array}$ & $\begin{array}{l}68.5285 \\
67.6209 \\
66.6919 \\
65.7384\end{array}$ & $\begin{array}{l}.08027 \\
.07805 \\
.07580 \\
.07353\end{array}$ & $\begin{array}{r}106.51 \\
100.07 \\
94.00 \\
88.35\end{array}$ & $\begin{array}{l}.00286 \\
.00280 \\
.00273 \\
.00266\end{array}$ & $\begin{array}{l}180 . \\
185 . \\
190 . \\
195 .\end{array}$ & $\begin{array}{l}63.5868 \\
67.6936 \\
56.7594 \\
55.8116\end{array}$ & $\begin{array}{l}.08044 \\
.07823 \\
.07599 \\
.07374\end{array}$ & $\begin{array}{r}107.02 \\
100.47 \\
94.39 \\
88.73\end{array}$ & $\begin{array}{l}.00287 \\
.00281 \\
.00274 \\
.00267\end{array}$ \\
\hline $\begin{array}{l}200 . \\
205 . \\
210 . \\
215 . \\
220 . \\
225 . \\
230 . \\
235 . \\
240 . \\
245 .\end{array}$ & $\begin{array}{l}64.7570 \\
63.7435 \\
62.5979 \\
31.5988 \\
60.4534 \\
59.2465 \\
57.955 ? \\
56.5711 \\
55.0982 \\
53.4464\end{array}$ & $\begin{array}{l}.07126 \\
.04391 \\
.06654 \\
.06414 \\
.06169 \\
.05918 \\
.05652 \\
.05397 \\
.05123 \\
.04838\end{array}$ & $\begin{array}{l}83.07 \\
78.13 \\
73.47 \\
69.09 \\
64.90 \\
60.91 \\
57.08 \\
53.36 \\
49.71 \\
46.08\end{array}$ & $\begin{array}{l}.00259 \\
.00251 \\
.00242 \\
.00233 \\
.00224 \\
.00213 \\
.00202 \\
.00190 \\
.00176 \\
.00161\end{array}$ & $\begin{array}{l}200 . \\
205 . \\
211 . \\
215 . \\
220 . \\
225 . \\
230 . \\
235 . \\
240 . \\
245 .\end{array}$ & $\begin{array}{l}64.7366 \\
63.8306 \\
62.7886 \\
61.7049 \\
60.5720 \\
59.3805 \\
58.1184 \\
56.7692 \\
55.3009 \\
53.7052\end{array}$ & $\begin{array}{l}.07145 \\
.06914 \\
.06679 \\
.06440 \\
.06197 \\
.05949 \\
.05695 \\
.05434 \\
.05165 \\
.04885\end{array}$ & $\begin{array}{l}83.46 \\
78.51 \\
73.86 \\
69.47 \\
65.31 \\
61.33 \\
57.52 \\
53.82 \\
50.21 \\
46.64\end{array}$ & $\begin{array}{l}.00260 \\
.00252 \\
.00244 \\
.00235 \\
.00225 \\
.00215 \\
.00204 \\
.00192 \\
.00179 \\
.00165\end{array}$ \\
\hline $\begin{array}{l}250 . \\
251.551 \\
251.561 \\
255 . \\
260 . \\
265 . \\
270 . \\
275 . \\
280 . \\
285 . \\
290 . \\
295 .\end{array}$ & $\begin{array}{l}51.5673 \\
50.9160 \\
7.1021 \\
6.8402 \\
6.4474 \\
6.1709 \\
5.8456 \\
5.0077 \\
5.3984 \\
5.2115 \\
5.0428 \\
4.8803\end{array}$ & $\begin{array}{l}.04537 \\
.04439 \\
.00590 \\
.00979 \\
.00984 \\
.00593 \\
.01005 \\
.01023 \\
.01033 \\
.01025 \\
.01024 \\
.01028\end{array}$ & $\begin{array}{r}42.38 \\
41.19 \\
9.10 \\
8.14 \\
8.20 \\
8.29 \\
8.37 \\
8.47 \\
8.57 \\
8.47 \\
8.77 \\
8.88\end{array}$ & $\begin{array}{l}.00143 \\
.00137 \\
.00258 \\
.00301 \\
.00359 \\
.00416 \\
.00471 \\
.00527 \\
.00578 \\
.00616 \\
.00657 \\
.00699\end{array}$ & $\begin{array}{l}250 . \\
255 . \\
\text { * } 256.473 \\
* 256.473 \\
250 . \\
255 . \\
270 . \\
275 . \\
280 . \\
285 . \\
290 . \\
295 .\end{array}$ & $\begin{array}{l}51.8986 \\
49.7834 \\
49.0725 \\
8.3557 \\
7.8824 \\
7.3665 \\
6.9605 \\
6.6252 \\
6.3396 \\
6.0909 \\
5.8708 \\
5.6735\end{array}$ & $\begin{array}{l}.04592 \\
.04282 \\
.04186 \\
.01064 \\
.01058 \\
.01058 \\
.01067 \\
.01083 \\
.01089 \\
.01070 \\
.01063 \\
.01061\end{array}$ & $\begin{array}{r}43.02 \\
39.26 \\
38.09 \\
8.49 \\
8.49 \\
9.53 \\
8.59 \\
8.67 \\
8.75 \\
8.84 \\
8.93 \\
9.03\end{array}$ & $\begin{array}{l}.00148 \\
.00128 \\
.00121 \\
.00207 \\
.00252 \\
.00311 \\
.00367 \\
.00423 \\
.00472 \\
.00508 \\
.00546 \\
.00585\end{array}$ \\
\hline $\begin{array}{l}300 . \\
310 . \\
320 . \\
330 . \\
340 . \\
350 . \\
360 . \\
370 . \\
390 . \\
390 .\end{array}$ & $\begin{array}{l}4.7485 \\
4.4979 \\
4.2802 \\
4.0883 \\
3.9170 \\
3.7628 \\
3.6227 \\
3.4946 \\
3.3768 \\
3.2681\end{array}$ & $\begin{array}{l}.01034 \\
.01051 \\
.01071 \\
.01092 \\
.01115 \\
.01138 \\
.01162 \\
.01186 \\
.01211 \\
.01235\end{array}$ & $\begin{array}{r}8.99 \\
9.21 \\
9.43 \\
9.65 \\
9.87 \\
10.10 \\
10.32 \\
10.84 \\
10.76 \\
10.98\end{array}$ & $\begin{array}{l}.00742 \\
.00828 \\
.00915 \\
.01003 \\
.01092 \\
.01181 \\
.01272 \\
.01363 \\
.01455 \\
.01548\end{array}$ & $\begin{array}{l}300 . \\
310 . \\
320 . \\
330 . \\
340 . \\
350 . \\
360 . \\
370 . \\
380 . \\
390 .\end{array}$ & $\begin{array}{l}5.4948 \\
5.1817 \\
4.9142 \\
4.6811 \\
4.4751 \\
4.2910 \\
4.1249 \\
3.9738 \\
3.8356 \\
3.7084\end{array}$ & $\begin{array}{l}.01064 \\
.01076 \\
.01092 \\
.01112 \\
.01133 \\
.011155 \\
.01178 \\
.01201 \\
.01225 \\
.01248\end{array}$ & $\begin{array}{r}9.13 \\
9.34 \\
9.55 \\
9.76 \\
9.97 \\
10.19 \\
10.41 \\
10.63 \\
10.84 \\
11.06\end{array}$ & $\begin{array}{l}.00624 \\
.00703 \\
.00783 \\
.00864 \\
.00945 \\
.01026 \\
.01108 \\
.01190 \\
.01274 \\
.01358\end{array}$ \\
\hline $\begin{array}{l}400 . \\
410 . \\
420 . \\
430 . \\
440 . \\
450 . \\
460 . \\
470 . \\
480 . \\
490 .\end{array}$ & $\begin{array}{l}3.1671 \\
3.0732 \\
2.9853 \\
2.9030 \\
2.8756 \\
2.7528 \\
2.6839 \\
2.6189 \\
2.5571 \\
2.4985\end{array}$ & $\begin{array}{l}.01259 \\
.01294 \\
.01308 \\
.01332 \\
.01356 \\
.01381 \\
.01405 \\
.01479 \\
.01453 \\
.01477\end{array}$ & $\begin{array}{l}11.20 \\
11.42 \\
11.63 \\
11.85 \\
12.06 \\
12.27 \\
12.48 \\
12.89 \\
12.90 \\
13.11\end{array}$ & $\begin{array}{l}.01643 \\
.01738 \\
.01835 \\
.01933 \\
.02032 \\
.02132 \\
.02233 \\
.02336 \\
.02440 \\
.02545\end{array}$ & $\begin{array}{l}400 . \\
420 . \\
420 . \\
430 . \\
440 . \\
450 . \\
460 . \\
470 . \\
480 . \\
490 .\end{array}$ & $\begin{array}{l}3.5908 \\
3.4816 \\
3.3798 \\
3.2646 \\
3.1954 \\
3.1114 \\
3.0323 \\
2.9576 \\
2.8868 \\
2.8197\end{array}$ & $\begin{array}{l}.01272 \\
.01296 \\
.01320 \\
.01344 \\
.01368 \\
.01392 \\
.01416 \\
.01440 \\
.01463 \\
.01487\end{array}$ & $\begin{array}{l}11.27 \\
11.49 \\
11.70 \\
11.91 \\
12.12 \\
12.33 \\
12.54 \\
12.75 \\
12.95 \\
13.16\end{array}$ & $\begin{array}{l}.01443 \\
.01529 \\
.01616 \\
.01704 \\
.01793 \\
.01883 \\
.01974 \\
.02066 \\
.02159 \\
.02254\end{array}$ \\
\hline $\begin{array}{l}500 . \\
510 . \\
520 . \\
530 . \\
540 .\end{array}$ & $\begin{array}{l}2.4427 \\
2.3895 \\
2.3389 \\
2.2905 \\
2.2443\end{array}$ & $\begin{array}{l}.01500 \\
.01524 \\
.01548 \\
.01571 \\
.01595\end{array}$ & $\begin{array}{l}13.31 \\
13.51 \\
13.72 \\
13.92 \\
14.11\end{array}$ & $\begin{array}{l}.02651 \\
.02759 \\
.02867 \\
.02977 \\
.03088\end{array}$ & $\begin{array}{l}500 . \\
510 . \\
520 . \\
530 . \\
540 .\end{array}$ & $\begin{array}{l}2.7560 \\
2.6953 \\
2.6375 \\
2.3823 \\
2.5296\end{array}$ & $\begin{array}{l}.01511 \\
.01534 \\
.01558 \\
.01581 \\
.01605\end{array}$ & $\begin{array}{l}13.36 \\
23.56 \\
13.76 \\
13.96 \\
14.16\end{array}$ & $\begin{array}{l}.02349 \\
.02445 \\
.02543 \\
.02641 \\
.02740\end{array}$ \\
\hline
\end{tabular}

* Two Phase Boundary 
Table 7. Transoort Propertles of Oxygen, Isobars, Engr. Units.

500 . osia Isobar

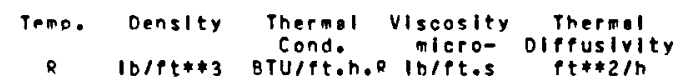

\begin{tabular}{|c|c|c|c|c|c|c|c|c|c|}
\hline $\begin{array}{l}98.558 \\
100 . \\
105 . \\
110 . \\
115 . \\
120 . \\
125 . \\
130 . \\
135 . \\
140 . \\
145 .\end{array}$ & $\begin{array}{l}P 1.6816 \\
81.4644 \\
80.7110 \\
79.9554 \\
79.1969 \\
78.4338 \\
77.6554 \\
74.8910 \\
75.1099 \\
75.3216 \\
74.5247\end{array}$ & $\begin{array}{l}.11617 \\
.11731 \\
.11446 \\
.11180 \\
.10927 \\
.10586 \\
.10453 \\
.10226 \\
.10005 \\
.09786 \\
.09570\end{array}$ & $\begin{array}{l}333.23 \\
327.24 \\
306.36 \\
285.81 \\
256.04 \\
247.32 \\
229.80 \\
213.51 \\
178.46 \\
184.80 \\
171.85\end{array}$ & $\begin{array}{l}.00364 \\
.00363 \\
.00357 \\
.00352 \\
.00347 \\
.00343 \\
.00339 \\
.00335 \\
.00331 \\
.00327 \\
.00323\end{array}$ & $\begin{array}{l}\text { * } 98.700 \\
100 . \\
105 . \\
110 . \\
115 . \\
120 . \\
125 . \\
130 . \\
135 . \\
140 . \\
145 .\end{array}$ & $\begin{array}{l}81.7091 \\
81.5136 \\
80.7617 \\
80.0083 \\
79.2519 \\
78.4913 \\
77.7258 \\
76.9546 \\
76.1768 \\
75.3920 \\
74.5993\end{array}$ & $\begin{array}{l}.11827 \\
.11749 \\
.11465 \\
.11199 \\
.10947 \\
.10706 \\
.10474 \\
.10248 \\
.10028 \\
.09810 \\
.09595\end{array}$ & $\begin{array}{l}334.65 \\
329.20 \\
308.16 \\
287.47 \\
267.57 \\
248.75 \\
231.13 \\
214.76 \\
199.63 \\
185.71 \\
172.91\end{array}$ & $\begin{array}{l}.00365 \\
.00363 \\
.00357 \\
.00352 \\
.00348 \\
.00344 \\
.00340 \\
.00336 \\
.00332 \\
.00328 \\
.00324\end{array}$ \\
\hline $\begin{array}{l}150 . \\
155 . \\
160 . \\
165 . \\
170 . \\
175 . \\
180 . \\
185 . \\
190 . \\
195 .\end{array}$ & $\begin{array}{l}73.7192 \\
72.9041 \\
72.0783 \\
71.2408 \\
70.3904 \\
69.5256 \\
69.6447 \\
67.7458 \\
66.8265 \\
65.8841\end{array}$ & $\begin{array}{l}.00355 \\
.09141 \\
.08027 \\
.08712 \\
.08497 \\
.08280 \\
.08751 \\
.07841 \\
.07519 \\
.07394\end{array}$ & $\begin{array}{l}160.16 \\
149.42 \\
139.57 \\
130.51 \\
122.18 \\
114.51 \\
107.42 \\
100.86 \\
94.78 \\
89.12\end{array}$ & $\begin{array}{l}.00318 \\
.00314 \\
.00309 \\
.00304 \\
.00299 \\
.00294 \\
.00288 \\
.00282 \\
.00275 \\
.00268\end{array}$ & $\begin{array}{l}150 . \\
155 . \\
160 . \\
165 . \\
170 . \\
175 . \\
180 . \\
185 . \\
190 . \\
195 .\end{array}$ & $\begin{array}{l}73.7982 \\
72.9877 \\
72.1670 \\
71.3351 \\
70.4909 \\
69.6328 \\
58.7594 \\
67.8688 \\
66.9589 \\
66.0271\end{array}$ & $\begin{array}{l}.09382 \\
.09169 \\
.08956 \\
.08742 \\
.08528 \\
.08312 \\
.08095 \\
.07877 \\
.07656 \\
.07434\end{array}$ & $\begin{array}{r}161.16 \\
150.38 \\
140.48 \\
131.39 \\
123.03 \\
115.33 \\
108.22 \\
101.65 \\
95.55 \\
89.88\end{array}$ & $\begin{array}{l}.00319 \\
.00315 \\
.00310 \\
.00306 \\
.00300 \\
.00295 \\
.00289 \\
.00283 \\
.00277 \\
.00270\end{array}$ \\
\hline $\begin{array}{l}200 . \\
205 \\
210 . \\
215 . \\
220 . \\
225 . \\
230 . \\
235 . \\
240 . \\
245 .\end{array}$ & $\begin{array}{l}64.9154 \\
63.9166 \\
62.8831 \\
61.8094 \\
60.6885 \\
59.5118 \\
58.2680 \\
56.942 ? \\
55.5138 \\
53.9523\end{array}$ & $\begin{array}{l}.07166 \\
.06936 \\
.06703 \\
.05466 \\
.06 ? 25 \\
.05979 \\
.05728 \\
.05470 \\
.05205 \\
.04931\end{array}$ & $\begin{array}{l}83.84 \\
78.89 \\
74.25 \\
59.86 \\
35.71 \\
61.75 \\
57.85 \\
54.28 \\
50.70 \\
47.18\end{array}$ & $\begin{array}{l}.00261 \\
.00753 \\
.00245 \\
.00236 \\
.00227 \\
.00217 \\
.00206 \\
.00195 \\
.00182 \\
.00168\end{array}$ & $\begin{array}{l}200 . \\
205 . \\
210 . \\
215 . \\
220 . \\
225 . \\
230 . \\
235 . \\
240 . \\
245 .\end{array}$ & $\begin{array}{l}65.0706 \\
64.0857 \\
63.0685 \\
62.0139 \\
60.9158 \\
59.7666 \\
58.5539 \\
57.2741 \\
55.9016 \\
54.4157\end{array}$ & $\begin{array}{l}.07209 \\
.05981 \\
.06750 \\
.06517 \\
.06279 \\
.06038 \\
.05792 \\
.05540 \\
.05283 \\
.05018\end{array}$ & $\begin{array}{l}84.60 \\
79.65 \\
75.01 \\
70.64 \\
66.50 \\
62.56 \\
58.79 \\
55.17 \\
51.65 \\
48.21\end{array}$ & $\begin{array}{l}.00263 \\
.00256 \\
.00248 \\
.00239 \\
.00230 \\
.00221 \\
.00210 \\
.00199 \\
.00188 \\
.00175\end{array}$ \\
\hline $\begin{array}{l}250 . \\
255 . \\
260 . \\
+260.997 \\
+260.907 \\
265 . \\
270 . \\
275 . \\
280 . \\
295 . \\
290 . \\
295 .\end{array}$ & $\begin{array}{l}52.2097 \\
50.2098 \\
47.7433 \\
47.1654 \\
9.4481 \\
8.9781 \\
8.2842 \\
7.7931 \\
7.3951 \\
7.0604 \\
9.7718 \\
6.518 ?\end{array}$ & $\begin{array}{l}.04645 \\
.04345 \\
.04027 \\
.03460 \\
.01171 \\
.01154 \\
.01151 \\
.01153 \\
.01161 \\
.01120 \\
.01109 \\
.01101\end{array}$ & $\begin{array}{l}43.64 \\
40.00 \\
36.08 \\
3.50 \\
8.92 \\
8.87 \\
8.88 \\
8.91 \\
8.97 \\
9.04 \\
9.12 \\
9.20\end{array}$ & $\begin{array}{l}.00152 \\
.00134 \\
.00111 \\
.00105 \\
.00165 \\
.00218 \\
.00278 \\
.00336 \\
.00386 \\
.00420 \\
.00456 \\
.00492\end{array}$ & $\begin{array}{l}250 . \\
255 . \\
260 . \\
265 . \\
+269.110 \\
+269.110 \\
270 . \\
275 . \\
280 . \\
285 . \\
290 . \\
295 .\end{array}$ & $\begin{array}{l}52.7814 \\
50.9415 \\
48.7905 \\
46.0857 \\
42.8802 \\
12.8674 \\
12.4697 \\
10.9554 \\
10.0494 \\
9.3905 \\
8.8712 \\
8.4425\end{array}$ & $\begin{array}{l}.04745 \\
.04462 \\
.04169 \\
.03865 \\
.03611 \\
.01516 \\
.01491 \\
.01435 \\
.01386 \\
.01291 \\
.01238 \\
.01208\end{array}$ & $\begin{array}{l}44.79 \\
41.36 \\
37.76 \\
33.76 \\
29.67 \\
10.02 \\
9.93 \\
9.67 \\
9.59 \\
9.57 \\
9.59 \\
9.63\end{array}$ & $\begin{array}{l}.00160 \\
.00144 \\
.00125 \\
.00101 \\
.00071 \\
.00093 \\
.00110 \\
.00186 \\
.00244 \\
.00281 \\
.00316 \\
.00351\end{array}$ \\
\hline $\begin{array}{l}300 . \\
310 . \\
320 . \\
330 . \\
340 . \\
350 . \\
360 . \\
370 . \\
380 . \\
390 .\end{array}$ & $\begin{array}{l}6.2922 \\
5.0033 \\
5.5771 \\
5.2947 \\
5.0516 \\
4.8344 \\
4.6377 \\
4.4536 \\
4.3033 \\
4.1564\end{array}$ & $\begin{array}{l}.01099 \\
.01104 \\
.01117 \\
.01134 \\
.01153 \\
.011173 \\
.01195 \\
.011217 \\
.01240 \\
.01263\end{array}$ & $\begin{array}{r}9.29 \\
9.48 \\
9.67 \\
9.88 \\
10.08 \\
10.29 \\
10.50 \\
10.72 \\
10.93 \\
11.14\end{array}$ & $\begin{array}{l}.00529 \\
.00604 \\
.00678 \\
.00752 \\
.00827 \\
.00902 \\
.00977 \\
.01052 \\
.01129 \\
.01206\end{array}$ & $\begin{array}{l}300 . \\
310 . \\
320 . \\
330 . \\
340 . \\
350 . \\
360 . \\
370 . \\
380 . \\
390 .\end{array}$ & $\begin{array}{l}8.0777 \\
7.4798 \\
7.0014 \\
3.6037 \\
6.2644 \\
5.0692 \\
5.7087 \\
5.4760 \\
5.2662 \\
5.0756\end{array}$ & $\begin{array}{l}.01190 \\
.01175 \\
.01176 \\
.011184 \\
.011197 \\
.01214 \\
.01232 \\
.01252 \\
.01272 \\
.01294\end{array}$ & $\begin{array}{r}9.68 \\
9.81 \\
9.97 \\
10.15 \\
10.33 \\
10.52 \\
10.72 \\
10.91 \\
11.11 \\
11.31\end{array}$ & $\begin{array}{l}.00385 \\
.00453 \\
.00519 \\
.00595 \\
.00650 \\
.00716 \\
.00781 \\
.00846 \\
.00912 \\
.00978\end{array}$ \\
\hline $\begin{array}{l}400 . \\
410 . \\
420 . \\
430 . \\
440 . \\
450 . \\
460 . \\
470 . \\
480 . \\
490 .\end{array}$ & $\begin{array}{l}4.0210 \\
3.8956 \\
3.7797 \\
3.3705 \\
3.5688 \\
3.4734 \\
3.3835 \\
3.2998 \\
3.2187 \\
3.1429\end{array}$ & $\begin{array}{l}.01286 \\
.01309 \\
.01333 \\
.01357 \\
.01390 \\
.01404 \\
.01147 \\
.01451 \\
.01474 \\
.01498\end{array}$ & $\begin{array}{l}11.35 \\
11.56 \\
11.77 \\
11.98 \\
12.19 \\
12.30 \\
12.60 \\
12.80 \\
13.61 \\
13.21\end{array}$ & $\begin{array}{l}.01283 \\
.01362 \\
.01441 \\
.01521 \\
.01602 \\
.01684 \\
.01767 \\
.01851 \\
.01935 \\
.02021\end{array}$ & $\begin{array}{l}400 . \\
410 . \\
420 . \\
430 . \\
440 . \\
450 . \\
400 . \\
470 . \\
480 . \\
490 .\end{array}$ & $\begin{array}{l}4.9012 \\
4.7409 \\
4.5026 \\
4.4550 \\
4.3268 \\
4.2069 \\
4.0944 \\
3.9987 \\
3.8890 \\
3.7948\end{array}$ & $\begin{array}{l}.01315 \\
.01337 \\
.01360 \\
.01382 \\
.01405 \\
.01428 \\
.01451 \\
.01474 \\
.01497 \\
.01520\end{array}$ & $\begin{array}{l}11.52 \\
11.72 \\
11.92 \\
12.12 \\
12.32 \\
12.52 \\
12.73 \\
12.92 \\
13.12 \\
13.32\end{array}$ & $\begin{array}{r}.01045 \\
.01112 \\
.01180 \\
.01248 \\
.01317 \\
.01387 \\
.01457 \\
.01528 \\
.01600 \\
.01672\end{array}$ \\
\hline $\begin{array}{l}500 . \\
510 . \\
520 . \\
530 . \\
540 .\end{array}$ & $\begin{array}{l}3.0709 \\
3.0024 \\
2.0373 \\
2.8752 \\
2.8159\end{array}$ & $\begin{array}{l}.01521 \\
.01545 \\
.01558 \\
.01591 \\
.01614\end{array}$ & $\begin{array}{l}13.41 \\
13.61 \\
13.81 \\
14.01 \\
14.20\end{array}$ & $\begin{array}{l}.02107 \\
.02195 \\
.02283 \\
.02372 \\
.02462\end{array}$ & $\begin{array}{l}500 . \\
510 . \\
520 . \\
530 . \\
540 .\end{array}$ & $\begin{array}{l}3.7057 \\
3.6211 \\
3.5407 \\
3.4642 \\
3.3913\end{array}$ & $\begin{array}{l}.01543 \\
.01566 \\
.01589 \\
.01612 \\
.01634\end{array}$ & $\begin{array}{l}13.52 \\
13.71 \\
13.91 \\
14.10 \\
14.30\end{array}$ & $\begin{array}{l}.01746 \\
.01819 \\
.01894 \\
.01969 \\
.02045\end{array}$ \\
\hline
\end{tabular}


Table 7. Transoopt proderties of Exvgen, Isobars, Enor. Units.

B00. OSI8 Isobar

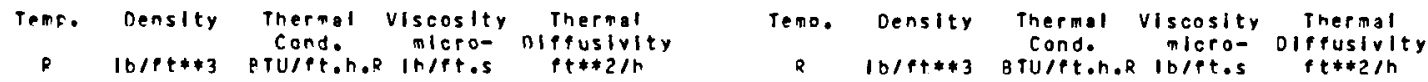

\begin{tabular}{|c|c|c|c|c|c|c|c|c|c|}
\hline $\begin{array}{l}09.841 \\
100 . \\
105 . \\
110 . \\
115 . \\
120 . \\
325 . \\
130 . \\
135 . \\
140 . \\
145 .\end{array}$ & $\begin{array}{l}41.7346 \\
81.5327 \\
80.8123 \\
90.0608 \\
79.3056 \\
78.5486 \\
77.7959 \\
77.0177 \\
74.2423 \\
75.4621 \\
74.6734\end{array}$ & $\begin{array}{l}.11836 \\
.11768 \\
.11484 \\
.11218 \\
.10966 \\
.10726 \\
.10495 \\
.10270 \\
.10050 \\
.09874 \\
.09520\end{array}$ & $\begin{array}{l}336.07 \\
331.17 \\
300.96 \\
289.13 \\
269.11 \\
250.19 \\
232.49 \\
216.01 \\
200.81 \\
186.81 \\
173.96\end{array}$ & $\begin{array}{l}.00365 \\
.00364 \\
.00358 \\
.00353 \\
.00348 \\
.00344 \\
.00340 \\
.00336 \\
.00332 \\
.00320 \\
.00325\end{array}$ & $\begin{array}{l}\text { * } 99.983 \\
100 . \\
105 . \\
110 . \\
115 . \\
120 . \\
125 . \\
130 . \\
135 . \\
140 .\end{array}$ & $\begin{array}{l}81.7640 \\
81.8116 \\
80.8627 \\
80.1130 \\
79.3610 \\
78.6056 \\
77.8456 \\
77.0905 \\
75.3094 \\
75.5317\end{array}$ & $\begin{array}{l}.11846 \\
.11786 \\
.11502 \\
.11237 \\
.10986 \\
.10747 \\
.10516 \\
.10292 \\
.10073 \\
.09858\end{array}$ & $\begin{array}{l}337.48 \\
333.14 \\
311.77 \\
290.79 \\
270.65 \\
251.61 \\
233.80 \\
217.26 \\
201.78 \\
187.92\end{array}$ & $\begin{array}{l}.00365 \\
.00334 \\
.00358 \\
.00353 \\
.00349 \\
.00345 \\
.00341 \\
.00337 \\
.00333 \\
.00329\end{array}$ \\
\hline $\begin{array}{l}150 . \\
155 . \\
160 . \\
165 . \\
170 . \\
175 . \\
180 . \\
185 . \\
190 . \\
190^{\circ}\end{array}$ & $\begin{array}{l}73.9765 \\
73.0706 \\
72.2549 \\
71.4285 \\
70.5902 \\
69.7387 \\
68.8726 \\
67.9901 \\
37.0892 \\
6.6 .1576\end{array}$ & $\begin{array}{l}.09407 \\
.09196 \\
.08984 \\
.08772 \\
.08559 \\
.08345 \\
.08129 \\
.07912 \\
.07694 \\
.07473\end{array}$ & $\begin{array}{l}162.16 \\
151.33 \\
141.39 \\
132.27 \\
123.88 \\
116.15 \\
109.02 \\
102.43 \\
96.32 \\
90.64\end{array}$ & $\begin{array}{l}.00320 \\
.00316 \\
.02321 \\
.00307 \\
.00302 \\
.00297 \\
.00291 \\
.00285 \\
.00270 \\
.00272\end{array}$ & $\begin{array}{l}165 . \\
150 . \\
155 . \\
160 . \\
165 . \\
170 . \\
175 . \\
180 . \\
185 . \\
190 .\end{array}$ & $\begin{array}{l}74.7469 \\
73.9541 \\
73.1528 \\
72.3470 \\
71.5209 \\
70.6884 \\
59.8434 \\
58.9443 \\
39.1096 \\
67.2175\end{array}$ & $\begin{array}{l}.09645 \\
.09433 \\
.09223 \\
.09012 \\
.08801 \\
.08589 \\
.08377 \\
.08183 \\
.07948 \\
.07731\end{array}$ & $\begin{array}{l}175.01 \\
133.15 \\
152.28 \\
142.31 \\
133.15 \\
124.72 \\
116.97 \\
109.82 \\
103.21 \\
97.08\end{array}$ & $\begin{array}{l}.00325 \\
.00321 \\
.00317 \\
.00313 \\
.00308 \\
.00303 \\
.00298 \\
.00292 \\
.00237 \\
.00281\end{array}$ \\
\hline $\begin{array}{l}200 . \\
205 . \\
210 \\
215 \\
220 \\
225 \\
230 \\
235 \\
240 . \\
245 .\end{array}$ & $\begin{array}{l}65.2227 \\
64.2511 \\
63.2493 \\
62.2126 \\
61.1358 \\
60.0121 \\
58.8334 \\
57.5892 \\
56.2658 \\
54.8446\end{array}$ & $\begin{array}{l}.07250 \\
.07025 \\
.05797 \\
.06566 \\
.06332 \\
.06095 \\
.05854 \\
.05608 \\
.05357 \\
.05100\end{array}$ & $\begin{array}{l}95.35 \\
80.41 \\
75.77 \\
71.40 \\
47.27 \\
63.35 \\
57.61 \\
56.83 \\
52.55 \\
49.19\end{array}$ & $\begin{array}{l}.00765 \\
.00258 \\
.00250 \\
.00242 \\
.00233 \\
.00224 \\
.00214 \\
.00204 \\
.00193 \\
.00181\end{array}$ & $\begin{array}{l}195 . \\
200 . \\
205 . \\
210 . \\
215 . \\
220 . \\
225 . \\
230 . \\
235 . \\
240 .\end{array}$ & $\begin{array}{l}66.3057 \\
55.3719 \\
64.4130 \\
63.4258 \\
62.4060 \\
51.3491 \\
50.2490 \\
59.0988 \\
57.8895 \\
56.3097\end{array}$ & $\begin{array}{l}.07512 \\
.07291 \\
.07068 \\
.06843 \\
.06815 \\
.06384 \\
.06151 \\
.05914 \\
.05673 \\
.05428\end{array}$ & $\begin{array}{l}91.39 \\
85.10 \\
81.15 \\
75.51 \\
72.15 \\
58.03 \\
64.13 \\
60.41 \\
55.85 \\
53.44\end{array}$ & $\begin{array}{l}.00274 \\
.00267 \\
.00260 \\
.00253 \\
.00245 \\
.00236 \\
.00228 \\
.00218 \\
.00208 \\
.00198\end{array}$ \\
\hline $\begin{array}{l}250 . \\
255 . \\
260 . \\
285 . \\
27 c . \\
275 . \\
+276.207 \\
+274.207 \\
280 . \\
285 . \\
290 . \\
295 .\end{array}$ & $\begin{array}{l}53.2990 \\
51.5887 \\
49.6466 \\
47.3439 \\
44.3657 \\
39.2588 \\
36.2669 \\
18.6292 \\
14.5266 \\
12.7045 \\
11.6137 \\
10.8277\end{array}$ & $\begin{array}{l}.04837 \\
.04567 \\
.04292 \\
.04014 \\
.03748 \\
.03552 \\
.03538 \\
.02519 \\
.01983 \\
.01587 \\
.014447 \\
.01368\end{array}$ & $\begin{array}{l}45.67 \\
42.55 \\
39.18 \\
35.6 ? \\
31.59 \\
25.24 \\
23.01 \\
12.20 \\
10.87 \\
10.46 \\
10.30 \\
10.23\end{array}$ & $\begin{array}{l}.00168 \\
.00153 \\
.00137 \\
.00118 \\
.00093 \\
.00049 \\
.00023 \\
.00025 \\
.00109 \\
.00165 \\
.00209 \\
.00245\end{array}$ & $\begin{array}{l}265 . \\
250 . \\
255 . \\
260 . \\
265 . \\
270 . \\
275 . \\
280 . \\
285 . \\
290 . \\
295 . \\
300 .\end{array}$ & $\begin{array}{l}55.2445 \\
53.7733 \\
52.1667 \\
50.3789 \\
48.3327 \\
45.8753 \\
42.6214 \\
36.7353 \\
19.7566 \\
15.8101 \\
14.0453 \\
12.9002\end{array}$ & $\begin{array}{l}.05178 \\
.04924 \\
.04665 \\
.04403 \\
.04141 \\
.03895 \\
.03706 \\
.03573 \\
.02479 \\
.01837 \\
.01628 \\
.01514\end{array}$ & $\begin{array}{l}50.12 \\
46.88 \\
43.57 \\
40.45 \\
37.15 \\
33.64 \\
29.58 \\
23.59 \\
12.96 \\
11.62 \\
11.18 \\
10.97\end{array}$ & $\begin{array}{l}.00185 \\
.00174 \\
.00161 \\
.00147 \\
.00130 \\
.00112 \\
.00088 \\
.00044 \\
.00052 \\
.00112 \\
.00157 \\
.00195\end{array}$ \\
\hline $\begin{array}{l}300 . \\
310 . \\
320 . \\
330 . \\
340 . \\
350 . \\
360 . \\
370 . \\
380 . \\
390 .\end{array}$ & $\begin{array}{r}10.2126 \\
9.2795 \\
8.5913 \\
8.0254 \\
7.5651 \\
7.1734 \\
6.8336 \\
6.5341 \\
6.2671 \\
6.0256\end{array}$ & $\begin{array}{l}.01320 \\
.01270 \\
.01250 \\
.01245 \\
.01251 \\
.01261 \\
.01275 \\
.01291 \\
.01308 \\
.01327\end{array}$ & $\begin{array}{l}10.21 \\
10.24 \\
10.34 \\
10.47 \\
10.62 \\
10.79 \\
10.94 \\
11.14 \\
11.32 \\
11.51\end{array}$ & $\begin{array}{l}.00279 \\
.00344 \\
.00406 \\
.00465 \\
.00525 \\
.00584 \\
.00642 \\
.00700 \\
.00758 \\
.00817\end{array}$ & $\begin{array}{l}310 . \\
320 . \\
330 . \\
340 . \\
350 . \\
360 . \\
370 . \\
380 . \\
390 . \\
400 .\end{array}$ & $\begin{array}{r}11.3817 \\
10.3546 \\
9.5818 \\
9.9649 \\
9.4535 \\
8.0181 \\
7.6401 \\
7.3071 \\
7.0101 \\
3.7427\end{array}$ & $\begin{array}{l}.01398 \\
.01346 \\
.01323 \\
.01315 \\
.01317 \\
.01324 \\
.01335 \\
.01349 \\
.01365 \\
.01382\end{array}$ & $\begin{array}{l}10.81 \\
10.80 \\
10.86 \\
10.97 \\
11.09 \\
11.24 \\
11.40 \\
11.56 \\
11.73 \\
11.91\end{array}$ & $\begin{array}{l}.00261 \\
.00321 \\
.00377 \\
.00432 \\
.00486 \\
.00539 \\
.00591 \\
.00644 \\
.00697 \\
.00749\end{array}$ \\
\hline $\begin{array}{l}400 . \\
410 . \\
420 . \\
430 . \\
440 . \\
450 . \\
460 . \\
470 . \\
480 . \\
490 .\end{array}$ & $\begin{array}{l}5.1084 \\
5.6090 \\
5.4257 \\
5.2584 \\
5.0993 \\
4.9530 \\
4.8183 \\
4.6881 \\
4.5676 \\
4.4541\end{array}$ & $\begin{array}{l}.01347 \\
.01368 \\
.01389 \\
.01416 \\
.01432 \\
.01454 \\
.01476 \\
.01498 \\
.01520 \\
.01543\end{array}$ & $\begin{array}{l}11.70 \\
11.89 \\
12.09 \\
12.28 \\
12.47 \\
12.67 \\
12.86 \\
13.03 \\
13.25 \\
13.44\end{array}$ & $\begin{array}{l}.00875 \\
.00934 \\
.00994 \\
.01054 \\
.01114 \\
.01175 \\
.01236 \\
.01298 \\
.01361 \\
.011424\end{array}$ & $\begin{array}{l}410 . \\
420 . \\
430 . \\
440 . \\
450 . \\
460 . \\
470 . \\
480 . \\
490 . \\
500 .\end{array}$ & $\begin{array}{l}6.5000 \\
5.2793 \\
6.0744 \\
5.8862 \\
5.7115 \\
5.5488 \\
5.3968 \\
5.2542 \\
5.1202 \\
4.9939\end{array}$ & $\begin{array}{l}.01401 \\
.011420 \\
.01440 \\
.01460 \\
.01481 \\
.01502 \\
.01523 \\
.01545 \\
.01567 \\
.01586\end{array}$ & $\begin{array}{l}12.07 \\
12.27 \\
12.45 \\
12.64 \\
12.83 \\
13.01 \\
13.20 \\
13.39 \\
13.57 \\
13.76\end{array}$ & $\begin{array}{l}.00802 \\
.00855 \\
.00909 \\
.00963 \\
.01017 \\
.01072 \\
.01127 \\
.01183 \\
.01239 \\
.01295\end{array}$ \\
\hline $\begin{array}{l}500 . \\
510 . \\
520 . \\
530 . \\
540 .\end{array}$ & $\begin{array}{l}4.3468 \\
4.2453 \\
4.1490 \\
4.0575 \\
3.9704\end{array}$ & $\begin{array}{l}.01565 \\
.01588 \\
.01610 \\
.01632 \\
.01655\end{array}$ & $\begin{array}{l}13.64 \\
13.83 \\
14.02 \\
14.21 \\
14.40\end{array}$ & $\begin{array}{l}.01488 \\
.01552 \\
.01617 \\
.01682 \\
.01748\end{array}$ & $\begin{array}{l}510 . \\
520 . \\
530 . \\
540 . \\
530 .\end{array}$ & $\begin{array}{l}4.8747 \\
4.7617 \\
4.6546 \\
4.5528 \\
3.4642\end{array}$ & $\begin{array}{l}.01610 \\
.01632 \\
.01654 \\
.01676 \\
.01612\end{array}$ & $\begin{array}{l}13.95 \\
14.13 \\
14.32 \\
14.51 \\
14.10\end{array}$ & $\begin{array}{l}.01352 \\
.01410 \\
.01468 \\
.01526 \\
.01969\end{array}$ \\
\hline
\end{tabular}


Table 7. Transport Prodertles of Oxyoen, Isobars, Engr. Unlts.

900. psi. Isober

1000. Osia Isobar

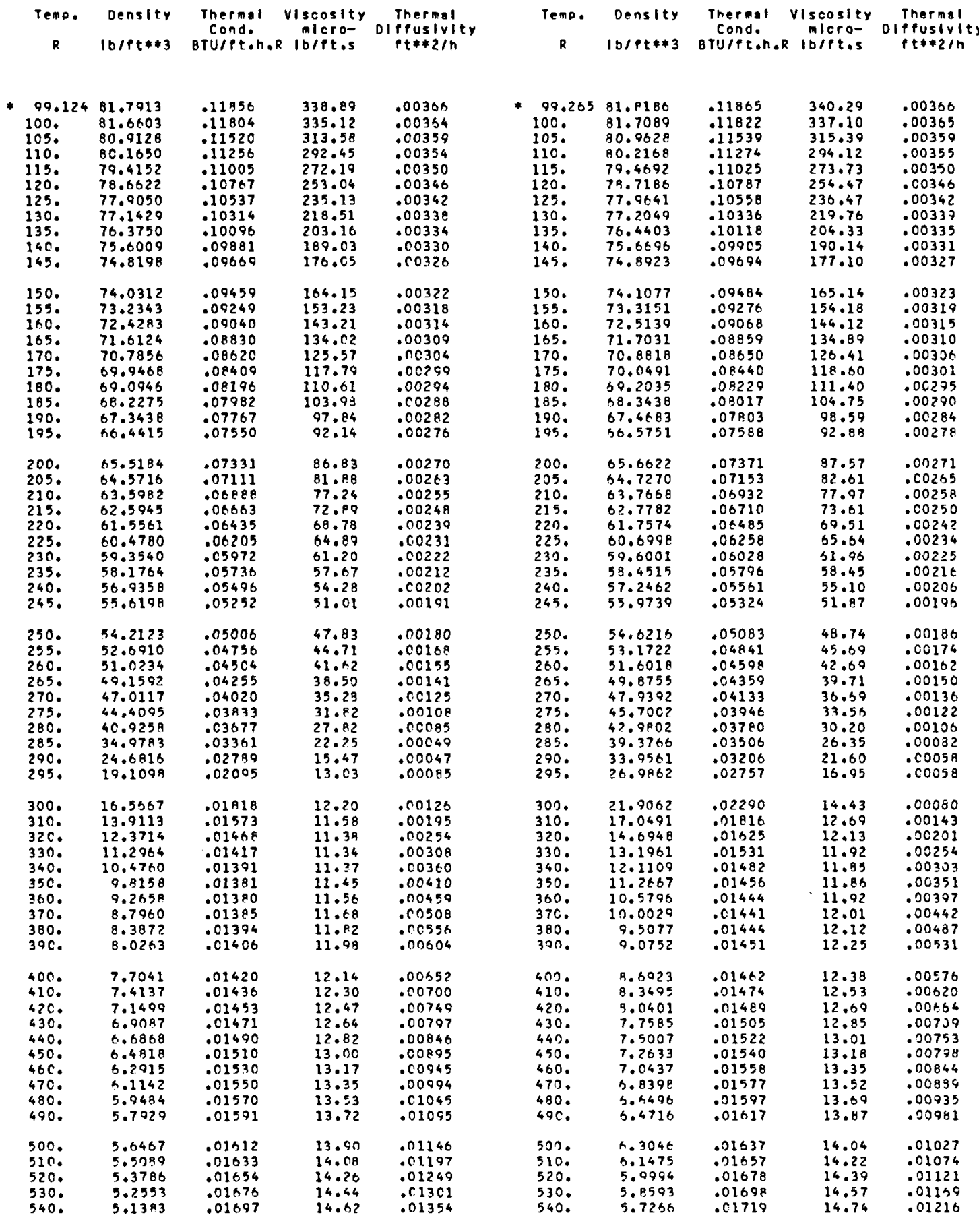


Table 7. Transport Propertles of Rxygen, Isobars, Engr. Unlts.

1100 . Osia isobar

1200. osla Isobar

\begin{tabular}{|c|c|c|c|c|c|c|c|c|c|}
\hline Teno. & $\begin{array}{l}\text { Density } \\
10 / f+* * 3\end{array}$ & $\begin{array}{l}\text { Thermal } \\
\text { Cond. } \\
\text { BTuleteh.R }\end{array}$ & $\begin{array}{l}\text { viscosity } \\
\text { micro- } \\
\text { lo/ftes }\end{array}$ & $\begin{array}{c}\text { Thermal } \\
\text { Diffusivity } \\
\text { ft } * 2 / h\end{array}$ & $\begin{array}{c}\text { Tenp. } \\
R\end{array}$ & $\begin{array}{l}\text { Density } \\
\text { Iotet } * 3\end{array}$ & $\begin{array}{l}\text { Thermal } \\
\text { Cond. } \\
\text { BTUlftoh.R }\end{array}$ & $\begin{array}{c}\text { VIscosity } \\
\text { mlcra- } \\
\text { Io/ftes }\end{array}$ & $\begin{array}{r}\text { Thermal } \\
\text { Diffusiy it } \\
\text { ft* } * 2 / \mathrm{h}\end{array}$ \\
\hline $\begin{array}{l}99.406 \\
100 . \\
165 . \\
110 . \\
115 . \\
126 . \\
125 . \\
130 . \\
135 . \\
140 . \\
145 .\end{array}$ & $\begin{array}{l}81.845 P \\
81.7573 \\
81.0126 \\
80.2684 \\
79.5229 \\
78.7747 \\
78.0229 \\
77.2666 \\
76.5051 \\
75.7379 \\
74.0542\end{array}$ & $\begin{array}{l}.11875 \\
.11840 \\
.11557 \\
.111293 \\
.11044 \\
.10807 \\
.10579 \\
.10357 \\
.10161 \\
.09928 \\
.09718\end{array}$ & $\begin{array}{l}241.09 \\
339.09 \\
317.21 \\
295.79 \\
275.27 \\
255.00 \\
237.80 \\
221.01 \\
205.51 \\
191.24 \\
178.15\end{array}$ & $\begin{array}{l}.00366 \\
.00365 \\
.00360 \\
.00355 \\
.00351 \\
.00347 \\
.00343 \\
.00339 \\
.00336 \\
.00332 \\
.00328\end{array}$ & $\begin{array}{l}* 99.547 \\
100 . \\
105 . \\
110 . \\
115 . \\
120 . \\
125 . \\
130 . \\
135 . \\
140 . \\
145 .\end{array}$ & $\begin{array}{l}81.8729 \\
81.8055 \\
81.0622 \\
80.3198 \\
79.5763 \\
78.8305 \\
78.0814 \\
77.3279 \\
76.5696 \\
75.8057 \\
75.0357\end{array}$ & $\begin{array}{l}.11885 \\
.11858 \\
.11575 \\
.11312 \\
.11064 \\
.10827 \\
.10599 \\
.10379 \\
.10163 \\
.09951 \\
.09742\end{array}$ & $\begin{array}{l}343.09 \\
341.09 \\
319.03 \\
297.47 \\
276.82 \\
257.34 \\
239.14 \\
222.26 \\
206.68 \\
192.35 \\
179.19\end{array}$ & $\begin{array}{l}.00366 \\
.00366 \\
.00360 \\
.00356 \\
.00351 \\
.00347 \\
.00344 \\
.00340 \\
.00336 \\
.00333 \\
.00329\end{array}$ \\
\hline $\begin{array}{l}150 . \\
1550 \\
160 . \\
1650 \\
170 . \\
1750 \\
1700 \\
1850 \\
190 . \\
195 .\end{array}$ & $\begin{array}{l}74.1836 \\
73.3953 \\
72.5986 \\
71.7928 \\
70.9770 \\
70.1502 \\
69.3111 \\
68.4585 \\
67.5909 \\
66.7066\end{array}$ & $\begin{array}{l}.09510 \\
.09302 \\
.09095 \\
.08888 \\
.08680 \\
.08471 \\
.08262 \\
.08051 \\
.07830 \\
.07626\end{array}$ & $\begin{array}{l}166.14 \\
155.13 \\
145.03 \\
135.76 \\
127.24 \\
119.41 \\
112.18 \\
105.51 \\
99.34 \\
93.62\end{array}$ & $\begin{array}{l}.00324 \\
.00320 \\
.00316 \\
.00311 \\
.00307 \\
.00302 \\
.00297 \\
.00291 \\
.00286 \\
.00280\end{array}$ & $\begin{array}{l}150 . \\
155^{\circ} \\
160 . \\
165 . \\
170 . \\
175 . \\
180 . \\
185 . \\
190 . \\
195 .\end{array}$ & $\begin{array}{l}74.2589 \\
73.4748 \\
77.6827 \\
71.8818 \\
71.0713 \\
70.2502 \\
69.4174 \\
68.5718 \\
67.7118 \\
66.8360\end{array}$ & $\begin{array}{l}.09535 \\
.09329 \\
.09122 \\
.08916 \\
.08710 \\
.08503 \\
.08294 \\
.08085 \\
.07875 \\
.07663\end{array}$ & $\begin{array}{l}167.13 \\
156.07 \\
145.93 \\
136.62 \\
128.08 \\
120.21 \\
112.96 \\
106.27 \\
100.09 \\
94.35\end{array}$ & $\begin{array}{l}.00325 \\
.00321 \\
.00317 \\
.00313 \\
.00308 \\
.00303 \\
.00298 \\
.00293 \\
.00287 \\
.00281\end{array}$ \\
\hline $\begin{array}{l}200 . \\
2050 \\
2100 \\
2150 \\
220 . \\
2250 \\
2300 \\
2350 \\
240 . \\
265 .\end{array}$ & $\begin{array}{l}65.8035 \\
64.8794 \\
63.931 \% \\
62.9575 \\
61.9533 \\
60.9149 \\
59.8377 \\
58.7159 \\
57.5426 \\
56.3094\end{array}$ & $\begin{array}{l}.07411 \\
.07194 \\
.05676 \\
.08756 \\
.06534 \\
.06310 \\
.06084 \\
.05855 \\
.05695 \\
.05392\end{array}$ & $\begin{array}{l}88.29 \\
83.33 \\
78.69 \\
74.33 \\
70.24 \\
66.37 \\
62.70 \\
59.22 \\
55.89 \\
52.70\end{array}$ & $\begin{array}{l}.00273 \\
.00267 \\
.00260 \\
.00253 \\
.00245 \\
.00237 \\
.00228 \\
.00220 \\
.00210 \\
.00201\end{array}$ & $\begin{array}{l}200 . \\
205 . \\
210 . \\
215 . \\
220 . \\
225 . \\
230 . \\
235 . \\
240 . \\
245 .\end{array}$ & $\begin{array}{l}65.9424 \\
65.0289 \\
64.0933 \\
63.1327 \\
62.1441 \\
61.1238 \\
60.0675 \\
58.9704 \\
57.8265 \\
53.6286\end{array}$ & $\begin{array}{l}.07450 \\
.07235 \\
.07019 \\
.06801 \\
.06582 \\
.06360 \\
.06138 \\
.05913 \\
.05686 \\
.05458\end{array}$ & $\begin{array}{l}89.01 \\
64.04 \\
79.39 \\
75.04 \\
70.95 \\
67.09 \\
63.44 \\
59.97 \\
56.66 \\
53.50\end{array}$ & $\begin{array}{l}.00275 \\
.00269 \\
.00262 \\
.00255 \\
.00248 \\
.00240 \\
.00232 \\
.00223 \\
.00214 \\
.00205\end{array}$ \\
\hline $\begin{array}{l}250 . \\
2550 \\
260 . \\
2650 \\
270 . \\
2750 \\
280 . \\
2850 \\
290 . \\
295\end{array}$ & $\begin{array}{l}55.0058 \\
53.6181 \\
52.1282 \\
50.5113 \\
48.7310 \\
46.7313 \\
44.4195 \\
41.0287 \\
38.0518 \\
33.3314\end{array}$ & $\begin{array}{l}.05158 \\
.04922 \\
.04696 \\
.04455 \\
.04234 \\
.04050 \\
.03879 \\
.03623 \\
.03370 \\
.03094\end{array}$ & $\begin{array}{l}49.61 \\
46.62 \\
43.70 \\
40.81 \\
37.94 \\
35.02 \\
32.01 \\
28.79 \\
25.22 \\
21.29\end{array}$ & $\begin{array}{l}.00191 \\
.00180 \\
.00169 \\
.00157 \\
.00145 \\
.00133 \\
.00120 \\
.00102 \\
.00083 \\
.00069\end{array}$ & $\begin{array}{l}250 . \\
255 . \\
260 . \\
265 . \\
270 . \\
275 . \\
280 . \\
285 . \\
290 . \\
295 .\end{array}$ & $\begin{array}{l}55.3682 \\
54.0341 \\
52.6126 \\
51.0851 \\
49.4261 \\
47.5994 \\
45.5501 \\
43.1929 \\
40.3929 \\
36.9726\end{array}$ & $\begin{array}{l}.03229 \\
.04999 \\
.04769 \\
.04544 \\
.04331 \\
.04146 \\
.03973 \\
.03731 \\
.03495 \\
.03260\end{array}$ & $\begin{array}{l}50.45 \\
47.51 \\
44.64 \\
41.84 \\
39.07 \\
36.31 \\
33.51 \\
30.63 \\
27.60 \\
24.38\end{array}$ & $\begin{array}{l}.00195 \\
.00185 \\
.00175 \\
.00164 \\
.00153 \\
.00143 \\
.00132 \\
.00117 \\
.00101 \\
.00087\end{array}$ \\
\hline $\begin{array}{l}300 . \\
310 . \\
320 . \\
330 . \\
340 . \\
350 . \\
360 . \\
370 . \\
380 . \\
390 .\end{array}$ & $\begin{array}{l}28.1152 \\
20.9261 \\
17.3850 \\
15.3058 \\
13.8505 \\
12.8108 \\
11.9515 \\
11.2614 \\
10.6584 \\
10.1560\end{array}$ & $\begin{array}{l}.02754 \\
.02131 \\
.01822 \\
.01670 \\
.01588 \\
.01542 \\
.01516 \\
.01503 \\
.01499 \\
.01501\end{array}$ & $\begin{array}{l}17.82 \\
14.30 \\
13.12 \\
12.63 \\
12.41 \\
12.33 \\
12.33 \\
12.37 \\
12.44 \\
12.54\end{array}$ & $\begin{array}{l}.00070 \\
.00108 \\
.00160 \\
.00211 \\
.00258 \\
.00303 \\
.00347 \\
.00389 \\
.00431 \\
.00472\end{array}$ & $\begin{array}{l}300 . \\
310 . \\
320 . \\
330 . \\
340 . \\
350 . \\
360 . \\
370 . \\
380 . \\
390 .\end{array}$ & $\begin{array}{l}32.9116 \\
25.2151 \\
20.4363 \\
17.6349 \\
15.7898 \\
14.4500 \\
13.4116 \\
12.5708 \\
11.8683 \\
11.2677\end{array}$ & $\begin{array}{l}.03013 \\
.02462 \\
.02055 \\
.01834 \\
.01712 \\
.01639 \\
.01597 \\
.01572 \\
.01559 \\
.01554\end{array}$ & $\begin{array}{l}21.15 \\
16.46 \\
14.40 \\
13.51 \\
13.09 \\
12.88 \\
12.79 \\
12.77 \\
12.80 \\
12.87\end{array}$ & $\begin{array}{l}.00079 \\
.00094 \\
.00132 \\
.00177 \\
.00222 \\
.00265 \\
.00306 \\
.00346 \\
.00385 \\
.00424\end{array}$ \\
\hline $\begin{array}{l}400 . \\
410 . \\
420 . \\
430 . \\
440 . \\
450 . \\
460 . \\
470 . \\
480 . \\
400 .\end{array}$ & $\begin{array}{l}9.7063 \\
9.3056 \\
0.9478 \\
8.6230 \\
8.3269 \\
8.0553 \\
7.8049 \\
7.5729 \\
7.3572 \\
7.1559\end{array}$ & $\begin{array}{l}.01507 \\
.01516 \\
.01577 \\
.01541 \\
.01556 \\
.01572 \\
.01589 \\
.01606 \\
.01624 \\
.01643\end{array}$ & $\begin{array}{l}12.66 \\
12.78 \\
12.92 \\
13.07 \\
13.22 \\
13.37 \\
13.53 \\
13.69 \\
13.86 \\
14.02\end{array}$ & $\begin{array}{l}.00514 \\
.00555 \\
.00596 \\
.00637 \\
.00679 \\
.00720 \\
.00762 \\
.00804 \\
.00846 \\
.00888\end{array}$ & $\begin{array}{l}400 . \\
410 . \\
420 . \\
430 . \\
440 . \\
450 . \\
460 . \\
470 . \\
480 . \\
490 .\end{array}$ & $\begin{array}{r}10.7450 \\
10.2837 \\
9.8721 \\
9.5013 \\
9.1646 \\
8.8569 \\
8.5742 \\
8.3129 \\
8.0706 \\
7.8440\end{array}$ & $\begin{array}{l}.01555 \\
.01560 \\
.01568 \\
.01579 \\
.01591 \\
.01605 \\
.01620 \\
.01636 \\
.01653 \\
.01670\end{array}$ & $\begin{array}{l}12.95 \\
13.06 \\
13.17 \\
13.30 \\
13.44 \\
13.58 \\
13.73 \\
13.88 \\
14.04 \\
14.19\end{array}$ & $\begin{array}{l}.00463 \\
.00502 \\
.00540 \\
.00578 \\
.00617 \\
.00655 \\
.00694 \\
.00733 \\
.00772 \\
.00811\end{array}$ \\
\hline $\begin{array}{l}500 . \\
510 . \\
520 . \\
530 . \\
540 .\end{array}$ & $\begin{array}{l}6.9673 \\
6.7902 \\
6.6234 \\
6.4660 \\
6.3171\end{array}$ & $\begin{array}{l}.01662 \\
.01682 \\
.01701 \\
.01721 \\
.01741\end{array}$ & $\begin{array}{l}14.19 \\
14.36 \\
14.53 \\
14.70 \\
14.87\end{array}$ & $\begin{array}{l}.00931 \\
.00974 \\
.01017 \\
.01061 \\
.01104\end{array}$ & $\begin{array}{l}500 . \\
510 . \\
520 . \\
530 . \\
540 .\end{array}$ & $\begin{array}{l}7.0340 \\
7.4363 \\
7.2504 \\
7.0752 \\
6.9097\end{array}$ & $\begin{array}{l}.01688 \\
.01707 \\
.01726 \\
.01745 \\
.01764\end{array}$ & $\begin{array}{l}14.35 \\
14.52 \\
14.68 \\
14.84 \\
15.01\end{array}$ & $\begin{array}{l}.00851 \\
.00891 \\
.00931 \\
.00971 \\
.01012\end{array}$ \\
\hline
\end{tabular}

* Tmo Phase Boundary 
Table 7. Transport Propertles of Oxygen, Isobars, Engr. Units.

1300. DSIE Isobsr

1400. PsIa Isobar

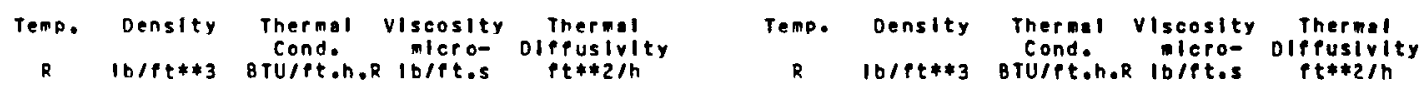

$169.11 \quad .00327$

$157.96 \quad .00323$

$147.73 \quad .00319$

$138.35 \quad .00315$

$129.73 \quad .00320$

$121.81 \quad .00306$

$114.52 \quad .00301$

$107.78 \quad .00296$

$\begin{array}{rr}101.56 & .00290 \\ 95.79 & .00285\end{array}$

$\begin{array}{ll}90.43 & .00279 \\ 05.45 & .00273\end{array}$

$85.45 \quad .00273$

$76.43 \quad .00260$

$72.34 \quad .00253$

$68.49 \quad .00245$

$04.85 \quad .00238$

64.050 .00238

$\begin{array}{ll}61.42 & .00230 \\ 58.15 & .00221\end{array}$

$\begin{array}{ll}58.15 & .00221 \\ 55.03 & .00213\end{array}$

$52.04 \quad .00204$

$49.17 \quad .00195$

$46.40 \quad .00186$

$43.72 \quad .00177$

$41.10 \quad .00167$

$38.54 \quad .00158$

$36.01 \quad .00149$

$33.49 \quad .00138$

$28.42 \quad .00115$

$25.86 \quad .00106$

$\begin{array}{ll}25.86 & .00106\end{array}$

$17.71 \quad .00111$

$15.80 \quad .00139$

$\begin{array}{ll}14.78 & .00173 \\ 14.22 & .00209\end{array}$

$13.90 \quad .00246$

$13.72 \quad .00282$

$\begin{array}{ll}13.63 & .00317 \\ 13.51 & .00352\end{array}$

$13.62 \quad .00386$

$13.74 \quad .00420$

$13.82 \quad .00488$

13.920 .0052

$13.92 \quad .00522$

$14.04 \quad .00556$

$14.16 \quad .00590$

$\begin{array}{ll}14.29 & .00624 \\ 14.42 & .00658\end{array}$

$14.56 \quad .00693$

$\begin{array}{lllll}500 . & 0.3343 & .01715 & 14.52 & .00784 \\ 510 . & 9.0553 & .01733 & 14.68 & .00821 \\ 520 . & 7.0709 & .01750 & 14.83 & .00858 \\ 530 . & 7.5944 & .01769 & 14.99 & .00896 \\ 540 . & 7.5039 & .01797 & 15.15 & .00934\end{array}$

$\begin{array}{ll}480 . & 9.5118 \\ 490 . & 9.2351\end{array}$

.01743

$500 . \quad 8.9775$

$\begin{array}{ll}510 . & 8.7367 \\ 520 . & 8.5111\end{array}$

$530 . \quad 8.2980$

.01759

$14.70 \quad .00727$

$14.85 \quad .00762$

$15.00 \quad .00797$

$\begin{array}{ll}15.15 & .00832 \\ 15.30 & .00867\end{array}$ 
Table 7. Transoort Propertles of Oxygen. Isobars, Engr. Units.

1500. psl. Isobar

1000. psite Isobar

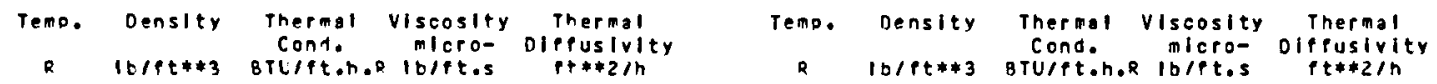

\begin{tabular}{|c|c|c|c|c|c|c|c|c|c|}
\hline $\begin{array}{l}99.969 \\
100 . \\
105 . \\
110 . \\
115 . \\
120 . \\
125 . \\
130 . \\
135 . \\
140 . \\
145 .\end{array}$ & $\begin{array}{l}81.9538 \\
81.9493 \\
81.2099 \\
80.4726 \\
79.7353 \\
78.9964 \\
78.2549 \\
77.5098 \\
76.7605 \\
76.0065 \\
75.2669\end{array}$ & $\begin{array}{l}.11913 \\
.11912 \\
.11630 \\
.11368 \\
.11121 \\
.10896 \\
.10361 \\
.10443 \\
.10230 \\
.10021 \\
.0914\end{array}$ & $\begin{array}{l}347.24 \\
347.10 \\
324.52 \\
302.51 \\
281.47 \\
261.65 \\
243.15 \\
226.01 \\
210.20 \\
195.67 \\
182.33\end{array}$ & $\begin{array}{l}.00367 \\
.00367 \\
.00362 \\
.00357 \\
.00353 \\
.00349 \\
.00344 \\
.00342 \\
.00339 \\
.00335 \\
.00331\end{array}$ & $\begin{array}{l}\text { * } 100.109 \\
105 . \\
110 . \\
115 . \\
120 . \\
125 . \\
130 . \\
135 . \\
140 . \\
145 .\end{array}$ & $\begin{array}{l}31.9806 \\
81.2588 \\
80.5231 \\
79.7877 \\
79.0511 \\
73.3121 \\
77.5698 \\
76.8235 \\
75.0725 \\
75.3164\end{array}$ & $\begin{array}{l}.11923 \\
.11648 \\
.11387 \\
.11140 \\
.10906 \\
.10681 \\
.10464 \\
.10252 \\
.10043 \\
.09838\end{array}$ & $\begin{array}{l}348.32 \\
326.36 \\
304.19 \\
283.03 \\
263.09 \\
244.49 \\
227.26 \\
211.38 \\
196.77 \\
183.37\end{array}$ & $\begin{array}{l}.00367 \\
.00362 \\
.00358 \\
.00354 \\
.00350 \\
.00346 \\
.00343 \\
.00339 \\
.00336 \\
.00332\end{array}$ \\
\hline $\begin{array}{l}150 . \\
155 . \\
160 \\
1650^{\circ} \\
170 \\
175 \circ \\
180 . \\
185 . \\
190^{\circ} \\
195 .\end{array}$ & $\begin{array}{l}74.4815 \\
73.7095 \\
72.9304 \\
72.1436 \\
71.3463 \\
70.5438 \\
69.7290 \\
68.9031 \\
68.0647 \\
67.2127\end{array}$ & $\begin{array}{l}.09610 \\
.09477 \\
.09204 \\
.09001 \\
.08798 \\
.08594 \\
.08390 \\
.08185 \\
.07979 \\
.07772\end{array}$ & $\begin{array}{l}170.10 \\
158.90 \\
148.63 \\
139.21 \\
130.56 \\
122.51 \\
115.29 \\
108.53 \\
102.29 \\
96.51\end{array}$ & $\begin{array}{l}.00328 \\
.00324 \\
.00320 \\
.00316 \\
.00312 \\
.00307 \\
.00302 \\
.00297 \\
.00292 \\
.00287\end{array}$ & $\begin{array}{l}150 . \\
255 . \\
150 . \\
165 . \\
170 . \\
175 . \\
180 . \\
185 . \\
190 . \\
195 .\end{array}$ & $\begin{array}{l}74.5546 \\
73.7865 \\
73.0116 \\
72.2293 \\
71.4389 \\
70.6396 \\
69.8306 \\
69.0108 \\
69.1793 \\
67.3347\end{array}$ & $\begin{array}{l}.09635 \\
.09432 \\
.09230 \\
.09029 \\
.08827 \\
.08624 \\
.08421 \\
.08217 \\
.08013 \\
.07807\end{array}$ & $\begin{array}{r}171.09 \\
159.84 \\
149.52 \\
140.07 \\
131.38 \\
123.40 \\
116.00 \\
109.28 \\
103.02 \\
97.22\end{array}$ & $\begin{array}{l}.00329 \\
.00325 \\
.00321 \\
.00317 \\
.00313 \\
.00309 \\
.00304 \\
.00299 \\
.00294 \\
.00288\end{array}$ \\
\hline $\begin{array}{l}200 . \\
205 \\
210 \\
215 \\
220 \\
225 \\
230 \\
2350 \\
240 . \\
245\end{array}$ & $\begin{array}{l}66.3455 \\
65.4616 \\
64.5590 \\
63.6356 \\
62.6892 \\
61.7171 \\
60.7162 \\
59.6833 \\
58.6143 \\
57.5048\end{array}$ & $\begin{array}{l}.07563 \\
.07354 \\
.07144 \\
.06932 \\
.06720 \\
.06506 \\
.06292 \\
.06077 \\
.05860 \\
.05644\end{array}$ & $\begin{array}{l}91.14 \\
86.14 \\
81.48 \\
77.12 \\
73.03 \\
59.18 \\
65.55 \\
52.12 \\
59.86 \\
55.76\end{array}$ & $\begin{array}{l}.00281 \\
.00275 \\
.00268 \\
.00262 \\
.00255 \\
.00248 \\
.00240 \\
.00233 \\
.00225 \\
.00217\end{array}$ & $\begin{array}{l}200 . \\
205 . \\
210 . \\
2150 \\
220 . \\
2250 \\
230 . \\
2350 \\
240 . \\
245 .\end{array}$ & $\begin{array}{l}63.4757 \\
65.6009 \\
54.7084 \\
63.7964 \\
62.8627 \\
61.9049 \\
60.9204 \\
59.9061 \\
59.8586 \\
57.7740\end{array}$ & $\begin{array}{l}.07500 \\
.07393 \\
.07184 \\
.06975 \\
.06765 \\
.05553 \\
.06341 \\
.06129 \\
.05916 \\
.05702\end{array}$ & $\begin{array}{l}91.83 \\
86.83 \\
82.16 \\
77.79 \\
73.70 \\
69.86 \\
66.23 \\
62.81 \\
59.56 \\
56.48\end{array}$ & $\begin{array}{l}.00282 \\
.00277 \\
.00270 \\
.00264 \\
.00257 \\
.00250 \\
.00243 \\
.00236 \\
.00228 \\
.00220\end{array}$ \\
\hline $\begin{array}{l}250 . \\
255 . \\
260 . \\
265 . \\
270 . \\
275 . \\
280 . \\
285 . \\
290 . \\
295 .\end{array}$ & $\begin{array}{l}56.3497 \\
55.1430 \\
53.8773 \\
52.5442 \\
51.1331 \\
40.6310 \\
48.0216 \\
46.2842 \\
44.3932 \\
42.3189\end{array}$ & $\begin{array}{l}.05427 \\
.05211 \\
.04996 \\
.04786 \\
.04586 \\
.04405 \\
.04231 \\
.04014 \\
.03805 \\
.03604\end{array}$ & $\begin{array}{l}52.80 \\
49.96 \\
47.23 \\
44.59 \\
42.03 \\
39.53 \\
37.08 \\
34.67 \\
32.29 \\
29.92\end{array}$ & $\begin{array}{l}.00208 \\
.00200 \\
.00191 \\
.00182 \\
.00173 \\
.00165 \\
.00156 \\
.00146 \\
.00136 \\
.00126\end{array}$ & $\begin{array}{l}250 . \\
250^{\circ} \\
260 . \\
2650^{\circ} \\
270 . \\
2750^{\circ} \\
280 . \\
2850^{\circ} \\
290 . \\
295 .\end{array}$ & $\begin{array}{l}56.6480 \\
55.4754 \\
54.2503 \\
52.9560 \\
51.6141 \\
50.1850 \\
48.6668 \\
47.0452 \\
45.3035 \\
43.4229\end{array}$ & $\begin{array}{l}.05489 \\
.05276 \\
.05066 \\
.04860 \\
.04662 \\
.04483 \\
.04309 \\
.04099 \\
.03896 \\
.03701\end{array}$ & $\begin{array}{l}53.53 \\
50.72 \\
48.02 \\
45.42 \\
42.90 \\
40.46 \\
38.08 \\
35.76 \\
33.47 \\
31.23\end{array}$ & $\begin{array}{l}.00212 \\
.00204 \\
.00195 \\
.00187 \\
.00179 \\
.00171 \\
.00163 \\
.00153 \\
.00144 \\
.00135\end{array}$ \\
\hline $\begin{array}{l}300 . \\
310 . \\
320 . \\
330 . \\
340 . \\
350 . \\
360 . \\
370 . \\
380 . \\
390 .\end{array}$ & $\begin{array}{l}40.0347 \\
34.8761 \\
29.6303 \\
25.3207 \\
22.1526 \\
19.8495 \\
16.1201 \\
16.7684 \\
15.6746 \\
14.7641\end{array}$ & $\begin{array}{l}.03412 \\
.03047 \\
.02701 \\
.02390 \\
.02156 \\
.01996 \\
.01889 \\
.01819 \\
.017772 \\
.01741\end{array}$ & $\begin{array}{l}27.56 \\
23.07 \\
19.48 \\
17.16 \\
15.80 \\
15.01 \\
14.54 \\
14.26 \\
14.10 \\
14.02\end{array}$ & $\begin{array}{l}.00117 \\
.00108 \\
.00111 \\
.00131 \\
.00159 \\
.00191 \\
.00224 \\
.00258 \\
.00291 \\
.00324\end{array}$ & $\begin{array}{l}300 . \\
310 . \\
320 . \\
330 . \\
340 . \\
350 . \\
360 . \\
370 . \\
380 . \\
390 .\end{array}$ & $\begin{array}{l}41.3863 \\
36.8457 \\
32.0332 \\
27.7163 \\
24.03046 \\
21.7272 \\
19.7659 \\
18.2318 \\
16.9950 \\
15.9709\end{array}$ & $\begin{array}{l}.03514 \\
.03166 \\
.02846 \\
.02551 \\
.02307 \\
.02126 \\
.01999 \\
.01912 \\
.01852 \\
.01811\end{array}$ & $\begin{array}{l}29.02 \\
24.78 \\
21.17 \\
18.57 \\
16.91 \\
15.88 \\
15.25 \\
14.86 \\
14.61 \\
14.47\end{array}$ & $\begin{array}{l}.00127 \\
.00115 \\
.00115 \\
.00128 \\
.00150 \\
.00177 \\
.00208 \\
.00239 \\
.00270 \\
.00301\end{array}$ \\
\hline $\begin{array}{l}400 . \\
410 . \\
420 . \\
430 . \\
440 . \\
450 . \\
460 . \\
470 . \\
480 . \\
490 .\end{array}$ & $\begin{array}{r}13.9894 \\
13.3184 \\
12.7289 \\
12.2048 \\
11.7345 \\
11.3089 \\
10.9211 \\
10.5656 \\
10.2381 \\
9.9348\end{array}$ & $\begin{array}{l}.01721 \\
.01710 \\
.01705 \\
.01705 \\
.01708 \\
.01714 \\
.01723 \\
.01733 \\
.01745 \\
.01758\end{array}$ & $\begin{array}{l}13.99 \\
14.01 \\
14.05 \\
14.11 \\
14.19 \\
14.28 \\
14.39 \\
14.50 \\
14.63 \\
14.75\end{array}$ & $\begin{array}{l}.00357 \\
.00389 \\
.00421 \\
.00453 \\
.00485 \\
.00517 \\
.00549 \\
.00581 \\
.00513 \\
.00646\end{array}$ & $\begin{array}{l}400 . \\
410 . \\
420 . \\
430 . \\
440 . \\
450 . \\
480 . \\
470 . \\
480 . \\
490 .\end{array}$ & $\begin{array}{l}15.1039 \\
14.3565 \\
13.7026 \\
13.1235 \\
12.6054 \\
12.1379 \\
11.7130 \\
11.3243 \\
10.9668 \\
10.6365\end{array}$ & $\begin{array}{l}.01783 \\
.01766 \\
.01756 \\
.01751 \\
.01751 \\
.01754 \\
.01760 \\
.01768 \\
.01778 \\
.01789\end{array}$ & $\begin{array}{l}14.39 \\
14.36 \\
14.37 \\
14.41 \\
14.47 \\
14.54 \\
14.63 \\
14.73 \\
14.84 \\
14.96\end{array}$ & $\begin{array}{l}.00332 \\
.00362 \\
.00393 \\
.00423 \\
.00453 \\
.00484 \\
.00514 \\
.00544 \\
.00575 \\
.00605\end{array}$ \\
\hline $\begin{array}{l}500 . \\
510 . \\
520 . \\
530 . \\
540 .\end{array}$ & $\begin{array}{l}9.6529 \\
9.3899 \\
9.1438 \\
8.9128 \\
8.6953\end{array}$ & $\begin{array}{l}.01772 \\
.01787 \\
.01802 \\
.01828 \\
.01835\end{array}$ & $\begin{array}{l}14.89 \\
15.03 \\
15.17 \\
15.31 \\
15.46\end{array}$ & $\begin{array}{l}.00678 \\
.00711 \\
.00744 \\
.00777 \\
.00810\end{array}$ & $\begin{array}{l}500 . \\
510 . \\
520 . \\
530 . \\
540 .\end{array}$ & $\begin{array}{r}10.3299 \\
10.0443 \\
9.7774 \\
9.5271 \\
9.2918\end{array}$ & $\begin{array}{l}.02802 \\
.01815 \\
.01829 \\
.01844 \\
.01859\end{array}$ & $\begin{array}{l}15.08 \\
15.21 \\
15.34 \\
15.48 \\
15.62\end{array}$ & $\begin{array}{l}.00636 \\
.00667 \\
.00698 \\
.00729 \\
.00760\end{array}$ \\
\hline
\end{tabular}

- Two Phase Boundery 
Toble 7. Transport Propertles of Oxygen, Isobars, Enor. Units.

1700. DSI. Isobar

Temp. Density Thermel viscosity thermel
R Ib/ft**3 BTU/ft.h.R ib/ftos Diffusirity

1800. ps Is Isobar

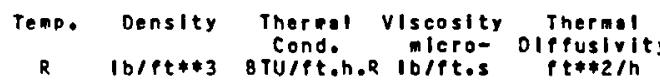

\begin{tabular}{|c|c|c|c|c|c|c|c|c|c|}
\hline $\begin{array}{l}100.250 \\
165 . \\
110 . \\
115 . \\
120 . \\
125 . \\
130 . \\
135 . \\
140 . \\
145 .\end{array}$ & $\begin{array}{l}82.0074 \\
81.3074 \\
80.5734 \\
79.8400 \\
79.1056 \\
78.3690 \\
77.6294 \\
76.8860 \\
76.1382 \\
75.3854\end{array}$ & $\begin{array}{l}.11933 \\
.11666 \\
.11405 \\
.11159 \\
.10926 \\
.10702 \\
.10485 \\
.10274 \\
.10066 \\
.09862\end{array}$ & $\begin{array}{l}349.99 \\
328.20 \\
305.88 \\
284.58 \\
264.53 \\
245.83 \\
228.52 \\
212.55 \\
197.87 \\
184.41\end{array}$ & $\begin{array}{l}.00368 \\
.00363 \\
.00358 \\
.00354 \\
.00350 \\
.00347 \\
.00343 \\
.00340 \\
.00337 \\
.00333\end{array}$ & $\begin{array}{l}100.390 \\
105 . \\
110 . \\
115 . \\
120 . \\
125 . \\
130 . \\
135 . \\
140 . \\
145 .\end{array}$ & $\begin{array}{l}82.0341 \\
61.3539 \\
80.6235 \\
79.8920 \\
79.1598 \\
78.4257 \\
77.6887 \\
76.9481 \\
76.2034 \\
75.4539\end{array}$ & $\begin{array}{l}.11942 \\
.11684 \\
.11423 \\
.11178 \\
.10945 \\
.10722 \\
.10506 \\
.10295 \\
.10089 \\
.09885\end{array}$ & $\begin{array}{l}351.36 \\
330.04 \\
307.57 \\
286.14 \\
265.97 \\
247.18 \\
229.77 \\
213.972 \\
198.98 \\
185.45\end{array}$ & $\begin{array}{l}.00368 \\
.00363 \\
.00359 \\
.00355 \\
.00351 \\
.00348 \\
.00344 \\
.00341 \\
.00337 \\
.00334\end{array}$ \\
\hline 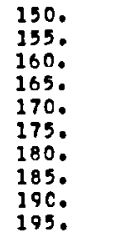 & $\begin{array}{l}74.6272 \\
73.8629 \\
73.0922 \\
72.3143 \\
71.5286 \\
70.7345 \\
69.9310 \\
69.1173 \\
68.2923 \\
67.4550\end{array}$ & $\begin{array}{l}.09659 \\
.09458 \\
.09257 \\
.09056 \\
.08855 \\
.08654 \\
.08452 \\
.08250 \\
.08046 \\
.07842\end{array}$ & $\begin{array}{l}172.08 \\
160.77 \\
150.42 \\
140.92 \\
132.21 \\
124.20 \\
116.82 \\
110.02 \\
103.74 \\
97.92\end{array}$ & $\begin{array}{l}.00330 \\
.00326 \\
.00322 \\
.00318 \\
.00314 \\
.00309 \\
.00305 \\
.00300 \\
.00295 \\
.00290\end{array}$ & $\begin{array}{l}130 . \\
155 . \\
160 . \\
165 . \\
170 . \\
175 . \\
180 . \\
185 . \\
190 . \\
195 .\end{array}$ & $\begin{array}{l}74.6992 \\
73.9388 \\
73.1720 \\
72.3985 \\
71.6179 \\
70.8284 \\
70.0303 \\
69.2225 \\
69.4039 \\
67.5736\end{array}$ & $\begin{array}{l}.09684 \\
.09483 \\
.09283 \\
.09084 \\
.08884 \\
.08684 \\
.08483 \\
.08282 \\
.08080 \\
.07877\end{array}$ & $\begin{array}{r}173.06 \\
161.71 \\
151.31 \\
141.78 \\
133.03 \\
124.98 \\
117.58 \\
110.76 \\
104.46 \\
98.62\end{array}$ & $\begin{array}{l}.00330 \\
.00327 \\
.00323 \\
.00319 \\
.00315 \\
.00311 \\
.00306 \\
.00301 \\
.00296 \\
.00291\end{array}$ \\
\hline $\begin{array}{l}200 . \\
205 . \\
210 . \\
2150 \\
220 \\
2250 \\
230 . \\
2350 \\
240 . \\
245 .\end{array}$ & $\begin{array}{l}66.0040 \\
65.7379 \\
64.8551 \\
63.9539 \\
63.0324 \\
62.0882 \\
61.1192 \\
60.1226 \\
59.0950 \\
58.0335\end{array}$ & $\begin{array}{l}.07637 \\
.07431 \\
.07224 \\
.07017 \\
.06808 \\
.06599 \\
.06390 \\
.06180 \\
.05949 \\
.05759\end{array}$ & $\begin{array}{l}92.53 \\
87.51 \\
92.83 \\
78.46 \\
74.37 \\
70.52 \\
66.90 \\
63.49 \\
60.25 \\
57.18\end{array}$ & $\begin{array}{l}.00284 \\
.00278 \\
.00272 \\
.00266 \\
.00260 \\
.00253 \\
.00246 \\
.00239 \\
.00231 \\
.00224\end{array}$ & $\begin{array}{l}200 . \\
205 . \\
210 . \\
2150^{\circ} \\
220 . \\
2250^{\circ} \\
230 . \\
2350^{\circ} \\
240 . \\
245 .\end{array}$ & $\begin{array}{l}66.7303 \\
65.8726 \\
64.9992 \\
64.1085 \\
63.1985 \\
62.2674 \\
61.3129 \\
60.3327 \\
59.3240 \\
58.2840\end{array}$ & $\begin{array}{l}.07673 \\
.07460 \\
.07264 \\
.07058 \\
.06852 \\
.06645 \\
.06437 \\
.06230 \\
.06022 \\
.05814\end{array}$ & $\begin{array}{l}93.21 \\
98.18 \\
83.50 \\
79.12 \\
75.03 \\
71.18 \\
67.56 \\
64.15 \\
60.92 \\
57.86\end{array}$ & $\begin{array}{l}.00286 \\
.00280 \\
.00274 \\
.00268 \\
.00262 \\
.00255 \\
.00248 \\
.00241 \\
.00234 \\
.00227\end{array}$ \\
\hline $\begin{array}{l}250 . \\
2550 \\
260 . \\
2650 \\
270 \\
2750 \\
280 . \\
2850 \\
290 \\
295 .\end{array}$ & $\begin{array}{l}56.9341 \\
55.7927 \\
54.6041 \\
53.3630 \\
52.0529 \\
50.6962 \\
49.2543 \\
47.7270 \\
46.1028 \\
44.3694\end{array}$ & $\begin{array}{l}.05549 \\
.05340 \\
.05133 \\
.04931 \\
.04736 \\
.04558 \\
.04385 \\
.04180 \\
.03983 \\
.03793\end{array}$ & $\begin{array}{l}34.25 \\
51.46 \\
48.78 \\
46.21 \\
43.74 \\
41.34 \\
39.02 \\
36.76 \\
34.56 \\
32.40\end{array}$ & $\begin{array}{l}.00216 \\
.00208 \\
.00200 \\
.00192 \\
.00184 \\
.00176 \\
.00169 \\
.00160 \\
.00151 \\
.00143\end{array}$ & $\begin{array}{l}250 . \\
255 . \\
250 . \\
2650^{\circ} \\
270^{\circ} \\
2750^{\circ} \\
280 . \\
2850^{\circ} \\
290 . \\
295 .\end{array}$ & $\begin{array}{l}57.2093 \\
56.0963 \\
54.9409 \\
53.7385 \\
52.4841 \\
51.1717 \\
49.7948 \\
48.3460 \\
46.8173 \\
45.2003\end{array}$ & $\begin{array}{l}.05607 \\
.05401 \\
.05198 \\
.04999 \\
.04807 \\
.04630 \\
.04458 \\
.04258 \\
.04065 \\
.03879\end{array}$ & $\begin{array}{l}54.95 \\
52.18 \\
49.52 \\
43.98 \\
44.54 \\
42.18 \\
39.90 \\
37.70 \\
35.56 \\
33.47\end{array}$ & $\begin{array}{l}.00219 \\
.00212 \\
.00204 \\
.00196 \\
.00189 \\
.00181 \\
.00174 \\
.00156 \\
.00158 \\
.00150\end{array}$ \\
\hline $\begin{array}{l}300 . \\
310 . \\
320 . \\
330 . \\
340 . \\
350 . \\
360 . \\
370 . \\
380 . \\
390 .\end{array}$ & $\begin{array}{l}42.5156 \\
38.4395 \\
34.0543 \\
29.8796 \\
26.3604 \\
23.5799 \\
21.4146 \\
19.7067 \\
18.3283 \\
17.1894\end{array}$ & $\begin{array}{l}.03310 \\
.03272 \\
.02967 \\
.02690 \\
.02448 \\
.02355 \\
.02111 \\
.02008 \\
.01935 \\
.01884\end{array}$ & $\begin{array}{l}30.30 \\
26.28 \\
22.72 \\
19.96 \\
18.06 \\
16.81 \\
16.01 \\
15.49 \\
15.18 \\
14.94\end{array}$ & $\begin{array}{l}.00135 \\
.00123 \\
.00120 \\
.00128 \\
.00145 \\
.00168 \\
.00195 \\
.00223 \\
.00252 \\
.00281\end{array}$ & $\begin{array}{l}300 . \\
310 . \\
320 . \\
330 . \\
340 . \\
350 . \\
360 . \\
370 . \\
380 . \\
390 .\end{array}$ & $\begin{array}{l}43.4976 \\
39.7679 \\
35.7569 \\
31.7947 \\
28.2751 \\
25.3690 \\
23.0407 \\
21.1749 \\
19.6640 \\
18.4127\end{array}$ & $\begin{array}{l}.03701 \\
.03370 \\
.03074 \\
.02810 \\
.02575 \\
.02377 \\
.02222 \\
.02106 \\
.02021 \\
.01959\end{array}$ & $\begin{array}{l}31.45 \\
27.50 \\
24.14 \\
21.31 \\
19.22 \\
17.78 \\
16.82 \\
16.17 \\
15.74 \\
15.45\end{array}$ & $\begin{array}{l}.00143 \\
.00131 \\
.00126 \\
.00130 \\
.00143 \\
.00162 \\
.00186 \\
.00211 \\
.00238 \\
.00265\end{array}$ \\
\hline $\begin{array}{l}400 . \\
410 . \\
420 . \\
430 . \\
440 . \\
450 . \\
460 . \\
470 . \\
480 . \\
490 .\end{array}$ & $\begin{array}{l}16.2284 \\
15.4029 \\
14.6832 \\
14.0477 \\
13.4808 \\
12.9705 \\
12.5077 \\
12.0852 \\
11.6973 \\
11.3395\end{array}$ & $\begin{array}{l}.01848 \\
.01824 \\
.01808 \\
.01799 \\
.01795 \\
.01795 \\
.01798 \\
.01803 \\
.01811 \\
.01821\end{array}$ & $\begin{array}{l}14.81 \\
14.75 \\
14.72 \\
14.73 \\
14.76 \\
14.82 \\
14.89 \\
14.97 \\
15.07 \\
15.17\end{array}$ & $\begin{array}{l}.00310 \\
.00339 \\
.00368 \\
.00397 \\
.00426 \\
.00455 \\
.00484 \\
.00512 \\
.00541 \\
.00570\end{array}$ & $\begin{array}{l}400 . \\
410 . \\
420 . \\
430 . \\
440 . \\
450 . \\
460 . \\
470 . \\
480 . \\
490 .\end{array}$ & $\begin{array}{l}17.3580 \\
16.4540 \\
15.6677 \\
14.9752 \\
14.3587 \\
13.8050 \\
13.3039 \\
12.8472 \\
12.4285 \\
12.0428\end{array}$ & $\begin{array}{l}.01915 \\
.01884 \\
.01862 \\
.01849 \\
.01840 \\
.01837 \\
.01837 \\
.01840 \\
.01846 \\
.01853\end{array}$ & $\begin{array}{l}15.26 \\
15.15 \\
15.08 \\
15.06 \\
15.07 \\
15.10 \\
15.15 \\
15.22 \\
15.30 \\
15.39\end{array}$ & $\begin{array}{l}.00293 \\
.00320 \\
.00348 \\
.00375 \\
.00402 \\
.00430 \\
.00457 \\
.00484 \\
.00512 \\
.00539\end{array}$ \\
\hline $\begin{array}{l}500 . \\
510 . \\
520 . \\
530 . \\
540 .\end{array}$ & $\begin{array}{r}11.0078 \\
10.6993 \\
10.4112 \\
10.1414 \\
9.8881\end{array}$ & $\begin{array}{l}.01831 \\
.01843 \\
.01856 \\
.01870 \\
.01894\end{array}$ & $\begin{array}{l}15.28 \\
15.40 \\
15.53 \\
15.65 \\
15.79\end{array}$ & $\begin{array}{l}.00599 \\
.00629 \\
.00658 \\
.00687 \\
.00717\end{array}$ & $\begin{array}{l}500 . \\
510 . \\
520 . \\
530 . \\
540 .\end{array}$ & $\begin{array}{l}11.6859 \\
11.3542 \\
11.0448 \\
10.7554 \\
10.4838\end{array}$ & $\begin{array}{l}.01862 \\
.01872 \\
.01884 \\
.01896 \\
.01909\end{array}$ & $\begin{array}{l}15.49 \\
15.80 \\
15.72 \\
15.83 \\
15.96\end{array}$ & $\begin{array}{l}.00567 \\
.00595 \\
.00623 \\
.00651 \\
.00679\end{array}$ \\
\hline
\end{tabular}


Table 7. Transport Properties of Oxygen, Isobars, Engr. Units.

1900. DsIa Isobar

2000. PSIa Isobar

Temo. Density Thermal Viscosity Thermal
Cond. micro- olffusivity

Temp. Density Thermal Viscosity Thermal

Cond. micro- Diffusivity

\begin{tabular}{|c|c|c|c|c|c|c|c|c|c|}
\hline $\begin{array}{l}100.529 \\
105 . \\
110 . \\
115 . \\
120 . \\
125 . \\
130 . \\
135 . \\
140 . \\
145 .\end{array}$ & $\begin{array}{l}82.0607 \\
81.4042 \\
80.6734 \\
79.9438 \\
79.2137 \\
78.4820 \\
77.7476 \\
77.0099 \\
76.2682 \\
75.5220\end{array}$ & $\begin{array}{l}.11952 \\
.11702 \\
.11442 \\
.11197 \\
.10945 \\
.10742 \\
.10527 \\
.10317 \\
.10111 \\
.09909\end{array}$ & $\begin{array}{l}352.72 \\
331.89 \\
309.26 \\
287.70 \\
267.42 \\
248.52 \\
231.02 \\
214.90 \\
200.08 \\
186.49\end{array}$ & $\begin{array}{l}.00368 \\
.00364 \\
.00359 \\
.00355 \\
.00352 \\
.00348 \\
.00345 \\
.00341 \\
.00338 \\
.00335\end{array}$ & $\begin{array}{l}+100.669 \\
105 . \\
110 . \\
115 . \\
120 . \\
125 . \\
130 . \\
135 . \\
140 . \\
145 .\end{array}$ & $\begin{array}{l}82.0872 \\
81.4524 \\
80.7231 \\
79.9953 \\
79.2674 \\
78.5360 \\
77.8063 \\
77.0713 \\
76.3326 \\
75.5896\end{array}$ & $\begin{array}{l}.11962 \\
.11720 \\
.11460 \\
.11216 \\
.10984 \\
.10762 \\
10548 \\
.10339 \\
.10134 \\
.09932\end{array}$ & $\begin{array}{l}354.08 \\
333.74 \\
310.96 \\
289.26 \\
268.86 \\
249.86 \\
232.27 \\
216.07 \\
201.18 \\
187.53\end{array}$ & $\begin{array}{l}.00368 \\
.00364 \\
.00360 \\
.00356 \\
.00352 \\
.00349 \\
.00345 \\
.00342 \\
.00339 \\
.00336\end{array}$ \\
\hline $\begin{array}{l}150 . \\
155 . \\
160 . \\
165 . \\
170 . \\
175 . \\
180 . \\
1850 \\
190 . \\
195 .\end{array}$ & $\begin{array}{l}74.7708 \\
74.0140 \\
73.2513 \\
72.4820 \\
71.7055 \\
70.9213 \\
70.1286 \\
69.3265 \\
68.5142 \\
67.4906\end{array}$ & $\begin{array}{l}.09708 \\
.09508 \\
.08310 \\
.09111 \\
.08912 \\
.08713 \\
.08514 \\
.08314 \\
.08113 \\
.07911\end{array}$ & $\begin{array}{l}174.05 \\
162.65 \\
152.20 \\
142.63 \\
133.84 \\
125.77 \\
118.34 \\
111.50 \\
105.17 \\
99.32\end{array}$ & $\begin{array}{l}.00331 \\
.00328 \\
.00324 \\
.00320 \\
.00316 \\
.00312 \\
.00307 \\
.00303 \\
.00298 \\
.00293\end{array}$ & $\begin{array}{l}150 . \\
155 . \\
160 . \\
165 . \\
170 . \\
175 . \\
180 . \\
185 . \\
190 . \\
195 .\end{array}$ & $\begin{array}{l}74.8418 \\
74.0887 \\
73.3299 \\
72.5647 \\
71.7928 \\
71.0134 \\
70.2258 \\
69.4293 \\
68.6231 \\
67.8062\end{array}$ & $\begin{array}{l}.09732 \\
.09534 \\
.09336 \\
.09138 \\
.08941 \\
.08743 \\
.08544 \\
.08345 \\
.08146 \\
.07946\end{array}$ & $\begin{array}{l}175.03 \\
163.58 \\
153.09 \\
143.48 \\
134.06 \\
126.55 \\
119.10 \\
112.23 \\
105.88 \\
100.02\end{array}$ & $\begin{array}{l}.00332 \\
.00329 \\
.00325 \\
.00321 \\
.00317 \\
.00313 \\
.00309 \\
.00304 \\
.00299 \\
.00294\end{array}$ \\
\hline $\begin{array}{l}200 . \\
205 \\
210 \\
2150 \\
220 \\
225 \\
230 \\
2350 \\
240 . \\
2450\end{array}$ & $\begin{array}{l}66.8548 \\
66.0053 \\
65.1409 \\
64.2601 \\
63.3612 \\
62.4425 \\
61.5019 \\
60.5373 \\
59.5463 \\
58.5263\end{array}$ & $\begin{array}{l}.07709 \\
.07506 \\
.07303 \\
.07099 \\
.06894 \\
.06689 \\
.06484 \\
.08279 \\
.06073 \\
.05868\end{array}$ & $\begin{array}{l}93.90 \\
88.86 \\
84.16 \\
79.78 \\
75.88 \\
71.83 \\
68.22 \\
64.81 \\
61.59 \\
58.53\end{array}$ & $\begin{array}{l}.00288 \\
.00282 \\
.00276 \\
.00270 \\
.00264 \\
.00258 \\
.00251 \\
.00244 \\
.00237 \\
.00230\end{array}$ & $\begin{array}{l}200 . \\
205 . \\
210 \\
215 \\
220 \\
225 \\
230 \\
235 \\
240 . \\
245 .\end{array}$ & $\begin{array}{l}66.9775 \\
66.1359 \\
65.2802 \\
64.4090 \\
63.5208 \\
62.6138 \\
61.6864 \\
60.7360 \\
59.7622 \\
58.7610\end{array}$ & $\begin{array}{l}.07745 \\
.07543 \\
.07341 \\
.07139 \\
.06936 \\
.06733 \\
.06530 \\
.06327 \\
.06124 \\
.05921\end{array}$ & $\begin{array}{l}94.58 \\
89.52 \\
84.82 \\
80.43 \\
76.32 \\
72.48 \\
68.86 \\
65.45 \\
62.24 \\
59.19\end{array}$ & $\begin{array}{l}.00289 \\
.00284 \\
.00278 \\
.00272 \\
.00266 \\
.00260 \\
.00253 \\
.00247 \\
.00240 \\
.00233\end{array}$ \\
\hline $\begin{array}{l}250 . \\
255 . \\
260 . \\
265 . \\
270 . \\
275 . \\
260 . \\
285 . \\
290 . \\
295 .\end{array}$ & $\begin{array}{l}57.4745 \\
56.3876 \\
55.2624 \\
54.0050 \\
52.9813 \\
51.8166 \\
50.2960 \\
48.9140 \\
47.4648 \\
45.9427\end{array}$ & $\begin{array}{l}.05684 \\
.05461 \\
.05261 \\
.05064 \\
.04875 \\
.04700 \\
.04529 \\
.04333 \\
.04144 \\
.03962\end{array}$ & $\begin{array}{l}55.64 \\
52.88 \\
50.24 \\
47.72 \\
45.30 \\
42.98 \\
40.74 \\
38.58 \\
36.49 \\
34.47\end{array}$ & $\begin{array}{l}.00223 \\
.00215 \\
.00208 \\
.00200 \\
.00193 \\
.00186 \\
.00179 \\
.00172 \\
.00164 \\
.00157\end{array}$ & $\begin{array}{l}250 . \\
255 . \\
260 . \\
265 . \\
270 . \\
275 . \\
280 . \\
285 . \\
290 . \\
295 .\end{array}$ & $\begin{array}{l}57.7304 \\
56.6678 \\
55.5703 \\
54.4346 \\
53.2575 \\
52.0353 \\
50.7641 \\
49.4397 \\
48.0581 \\
46.6151\end{array}$ & $\begin{array}{l}.05719 \\
.05519 \\
.05322 \\
.05128 \\
.04941 \\
.04767 \\
.04598 \\
.04405 \\
.04220 \\
.04041\end{array}$ & $\begin{array}{l}56.30 \\
53.56 \\
50.94 \\
48.44 \\
46.05 \\
43.75 \\
41.54 \\
39.42 \\
37.37 \\
35.39\end{array}$ & $\begin{array}{l}.00226 \\
.00219 \\
.00212 \\
.00204 \\
.00197 \\
.00191 \\
.00184 \\
.00177 \\
.00170 \\
.00163\end{array}$ \\
\hline $\begin{array}{l}300 . \\
310 . \\
320 . \\
330 . \\
340 . \\
350 . \\
360 . \\
370 . \\
380 . \\
390 .\end{array}$ & $\begin{array}{l}46.3427 \\
40.9037 \\
37.2070 \\
33.4765 \\
30.0281 \\
27.0657 \\
24.6204 \\
27.6240 \\
20.0911 \\
10.6331\end{array}$ & $\begin{array}{l}.03787 \\
.03462 \\
.03172 \\
.02916 \\
.02688 \\
.02491 \\
.02330 \\
.02203 \\
.02108 \\
.02037\end{array}$ & $\begin{array}{l}32.51 \\
28.79 \\
25.43 \\
22.58 \\
20.37 \\
18.77 \\
17.65 \\
16.88 \\
16.35 \\
15.98\end{array}$ & $\begin{array}{l}.00150 \\
.00139 \\
.00132 \\
.00134 \\
.00143 \\
.00159 \\
.00179 \\
.00202 \\
.00226 \\
.00252\end{array}$ & $\begin{array}{l}300 . \\
310 . \\
320 . \\
330 . \\
340 . \\
350 . \\
360 . \\
370 . \\
380 . \\
390 .\end{array}$ & $\begin{array}{l}45.1074 \\
41.8931 \\
38.4600 \\
34.9531 \\
31.6170 \\
28.6529 \\
26.1349 \\
24.0387 \\
22.2983 \\
20.8427\end{array}$ & $\begin{array}{l}.03870 \\
.03549 \\
.03264 \\
.03012 \\
.02790 \\
.02590 \\
.02432 \\
.02299 \\
.02194 \\
.02115\end{array}$ & $\begin{array}{l}33.48 \\
29.88 \\
26.60 \\
23.77 \\
21.48 \\
19.76 \\
18.50 \\
17.61 \\
16.98 \\
16.54\end{array}$ & $\begin{array}{l}.00156 \\
.00145 \\
.00138 \\
.00138 \\
.00145 \\
.00157 \\
.00175 \\
.00195 \\
.00217 \\
.00241\end{array}$ \\
\hline $\begin{array}{l}400 . \\
410 . \\
420 . \\
430 . \\
440 . \\
450 . \\
460 . \\
470 . \\
480 . \\
490 .\end{array}$ & $\begin{array}{l}18.6973 \\
17.5058 \\
16.6532 \\
15.9035 \\
15.2374 \\
14.6401 \\
14.1003 \\
13.6091 \\
13.1595 \\
12.7458\end{array}$ & $\begin{array}{l}.01984 \\
.01948 \\
.01919 \\
.01900 \\
.01887 \\
.01890 \\
.01877 \\
.01878 \\
.01891 \\
.01886\end{array}$ & $\begin{array}{l}15.73 \\
15.57 \\
15.47 \\
15.41 \\
15.39 \\
15.40 \\
15.43 \\
15.48 \\
15.55 \\
15.62\end{array}$ & $\begin{array}{l}.00278 \\
.00304 \\
.00330 \\
.00356 \\
.00382 \\
.00408 \\
.00434 \\
.00460 \\
.00496 \\
.00512\end{array}$ & $\begin{array}{l}400 . \\
410 . \\
420 . \\
430 . \\
440 . \\
450 . \\
460 . \\
470 . \\
480 . \\
490 .\end{array}$ & $\begin{array}{l}19.6106 \\
18.5541 \\
17.6364 \\
16.8303 \\
16.1147 \\
15.4739 \\
14.8955 \\
14.3699 \\
13.8893 \\
13.4475\end{array}$ & $\begin{array}{l}.02054 \\
.02009 \\
.01976 \\
.01952 \\
.01936 \\
.01925 \\
.01919 \\
.01916 \\
.01917 \\
.01920\end{array}$ & $\begin{array}{l}16.22 \\
16.01 \\
15.86 \\
15.77 \\
15.72 \\
15.71 \\
13.72 \\
15.75 \\
15.80 \\
15.86\end{array}$ & $\begin{array}{l}.00265 \\
.00290 \\
.00314 \\
.00339 \\
.00364 \\
.00389 \\
.00413 \\
.00438 \\
.00463 \\
.00488\end{array}$ \\
\hline $\begin{array}{l}500 . \\
510 . \\
520 . \\
530 . \\
540 .\end{array}$ & $\begin{array}{l}12.3633 \\
12.0083 \\
11.6776 \\
11.3684 \\
11.0784\end{array}$ & $\begin{array}{l}.01994 \\
.01902 \\
.01912 \\
.01923 \\
.01935\end{array}$ & $\begin{array}{l}15.71 \\
15.81 \\
15.91 \\
16.02 \\
16.14\end{array}$ & $\begin{array}{l}.00539 \\
.00565 \\
.00592 \\
.00618 \\
.00645\end{array}$ & $\begin{array}{l}500 . \\
510 . \\
520 . \\
530 . \\
540 .\end{array}$ & $\begin{array}{l}13.0395 \\
12.6611 \\
12.3089 \\
11.9799 \\
11.6716\end{array}$ & $\begin{array}{l}.01926 \\
.01933 \\
.01941 \\
.01951 \\
.01961\end{array}$ & $\begin{array}{l}15.93 \\
16.02 \\
16.11 \\
16.21 \\
16.32\end{array}$ & $\begin{array}{l}.00513 \\
.00539 \\
.00564 \\
.00589 \\
.00615\end{array}$ \\
\hline
\end{tabular}

* Tro phase Boundary 
Table 7. Transoort Propertles of Oxygen, Isobars, Engr. Units.

2200. D51. Isotar

2400. DSIA Isober

\begin{tabular}{|c|c|c|c|c|c|c|c|c|c|}
\hline $\begin{array}{c}\text { TeTr. } \\
\text { R }\end{array}$ & $\begin{array}{l}\text { Density } \\
16 / f t * * 3\end{array}$ & $\begin{array}{l}\text { Thermal } \\
\text { Cond. } \\
\text { BTulftoh,R }\end{array}$ & $\begin{array}{c}\text { VIscosity } \\
\text { micro- } \\
\text { Ibift.s }\end{array}$ & $\begin{array}{c}\text { Thermal } \\
\text { Diffusivity } \\
\text { ft } * 2 / h\end{array}$ & $\begin{array}{c}\text { Temp. } \\
R\end{array}$ & $\begin{array}{l}\text { Density } \\
10 /+t * * 3\end{array}$ & $\begin{array}{l}\text { Thermal } \\
\text { Cond. } \\
\text { B TUlft.h.R }\end{array}$ & $\begin{array}{c}\text { Viscosity } \\
\text { micro- } \\
\text { lo/ftos }\end{array}$ & $\begin{array}{c}\text { Thermel } \\
\text { oiffusivit } \\
\text { ft } \neq 2 / h\end{array}$ \\
\hline $\begin{array}{l}100.948 \\
105 . \\
110 . \\
115 . \\
120 . \\
125 . \\
130 . \\
135 . \\
140 . \\
143 .\end{array}$ & $\begin{array}{l}87.1400 \\
81.5481 \\
A 0.8219 \\
80.0978 \\
79.3740 \\
78.6493 \\
77.9226 \\
77.1931 \\
76.4602 \\
75.7235\end{array}$ & $\begin{array}{l}.11981 \\
.11756 \\
.11497 \\
.11253 \\
.11023 \\
.10902 \\
.10589 \\
.10392 \\
.10178 \\
.09978\end{array}$ & $\begin{array}{l}356.77 \\
337.46 \\
314.36 \\
292.39 \\
771.76 \\
252.55 \\
234.78 \\
218.42 \\
203.39 \\
189.61\end{array}$ & $\begin{array}{l}.00369 \\
.00365 \\
.00361 \\
.00357 \\
.00353 \\
.00350 \\
.00347 \\
.00344 \\
.00340 \\
.00337\end{array}$ & $\begin{array}{l}+101.226 \\
105 . \\
110 . \\
115 . \\
120 . \\
125 . \\
130 . \\
135 . \\
140 . \\
145 .\end{array}$ & $\begin{array}{l}82.1925 \\
81.6431 \\
80.9198 \\
80.1993 \\
79.4796 \\
78.7594 \\
78.0376 \\
77.3135 \\
76.5863 \\
75.8557\end{array}$ & $\begin{array}{l}.12000 \\
.11791 \\
.11533 \\
.11291 \\
.11061 \\
.10842 \\
.10630 \\
.10424 \\
.10223 \\
.10024\end{array}$ & $\begin{array}{l}359.45 \\
341.20 \\
317.78 \\
295.53 \\
274.66 \\
255.24 \\
237.29 \\
220.76 \\
205.59 \\
191.68\end{array}$ & $\begin{array}{l}.00369 \\
.00366 \\
.00362 \\
.00358 \\
.00354 \\
.00351 \\
.00348 \\
.00345 \\
.00342 \\
.00339\end{array}$ \\
\hline $\begin{array}{l}150 . \\
135 . \\
160 . \\
165 . \\
170 . \\
175 . \\
180 . \\
185 . \\
190 . \\
195 .\end{array}$ & $\begin{array}{l}74.9824 \\
74.2364 \\
73.4852 \\
72.7282 \\
71.9649 \\
71.1942 \\
70.4173 \\
69.6316 \\
68.8371 \\
68.0328\end{array}$ & $\begin{array}{l}.09780 \\
.09584 \\
.09388 \\
.09192 \\
.08996 \\
.08801 \\
.08605 \\
.08408 \\
.08211 \\
.08013\end{array}$ & $\begin{array}{l}176.99 \\
165.44 \\
154.86 \\
145.17 \\
136.28 \\
128.11 \\
120.60 \\
113.68 \\
107.30 \\
101.39\end{array}$ & $\begin{array}{l}.00334 \\
.00330 \\
.00327 \\
.00323 \\
.00319 \\
.00315 \\
.00311 \\
.00307 \\
.00302 \\
.00297\end{array}$ & $\begin{array}{l}150 . \\
155 . \\
160 . \\
165 . \\
170 . \\
175 . \\
180 . \\
185 . \\
190 . \\
195 .\end{array}$ & $\begin{array}{l}75.1210 \\
74.3820 \\
73.6381 \\
72.8890 \\
72.1340 \\
71.3729 \\
70.6049 \\
69.8296 \\
69.0461 \\
68.2538\end{array}$ & $\begin{array}{l}.09828 \\
.09633 \\
.09439 \\
.09245 \\
.09052 \\
.09858 \\
.08664 \\
.08469 \\
.08275 \\
.08079\end{array}$ & $\begin{array}{l}178.95 \\
167.30 \\
156.63 \\
146.86 \\
137.89 \\
129.66 \\
122.10 \\
115.13 \\
108.70 \\
102.76\end{array}$ & $\begin{array}{l}.00336 \\
.00332 \\
.00329 \\
.00325 \\
.00321 \\
.00318 \\
.00313 \\
.00309 \\
.00305 \\
.00300\end{array}$ \\
\hline $\begin{array}{l}200 . \\
205 . \\
210 . \\
215 . \\
220 . \\
225 . \\
230 . \\
235 \\
240 . \\
245 .\end{array}$ & $\begin{array}{l}67.2179 \\
63.3914 \\
65.5522 \\
64.6991 \\
63.8308 \\
62.9460 \\
62.0431 \\
61.1203 \\
60.1767 \\
59.2097\end{array}$ & $\begin{array}{l}.07815 \\
.07616 \\
.07417 \\
.07218 \\
.07018 \\
.06819 \\
.06619 \\
.06420 \\
.062 ? 1 \\
.06023\end{array}$ & $\begin{array}{l}95.92 \\
90.84 \\
86.12 \\
81.71 \\
77.59 \\
73.74 \\
70.12 \\
66.71 \\
63.50 \\
60.47\end{array}$ & $\begin{array}{l}.00292 \\
.00287 \\
.00282 \\
.00276 \\
.00270 \\
.00264 \\
.00258 \\
.00252 \\
.00245 \\
.00239\end{array}$ & $\begin{array}{l}200 . \\
205 \\
210 . \\
2150 \\
220 . \\
2250 \\
230 . \\
2350 \\
240 . \\
245 .\end{array}$ & $\begin{array}{l}67.4519 \\
66.6396 \\
65.8157 \\
64.9795 \\
64.1297 \\
63.2651 \\
62.3846 \\
61.4868 \\
60.5704 \\
59.6338\end{array}$ & $\begin{array}{l}.07884 \\
.07688 \\
.07491 \\
.07295 \\
.07099 \\
.06902 \\
.06706 \\
.06511 \\
.06316 \\
.06122\end{array}$ & $\begin{array}{l}97.25 \\
92.14 \\
87.40 \\
82.97 \\
78.84 \\
74.97 \\
71.35 \\
67.94 \\
64.74 \\
61.71\end{array}$ & $\begin{array}{l}.00295 \\
.00290 \\
.00285 \\
.00280 \\
.00274 \\
.00268 \\
.00262 \\
.00256 \\
.00250 \\
.00244\end{array}$ \\
\hline $\begin{array}{l}250 . \\
255 . \\
260 . \\
265 . \\
270 . \\
275 . \\
280 . \\
2850 \\
290 . \\
295 .\end{array}$ & $\begin{array}{l}58.2176 \\
57.1983 \\
56.1499 \\
55.0700 \\
53.9563 \\
52.8065 \\
51.6181 \\
50.3889 \\
49.1164 \\
47.7989\end{array}$ & $\begin{array}{l}.05827 \\
.05631 \\
.05430 \\
.05250 \\
.05068 \\
.04896 \\
.04729 \\
.04543 \\
.04364 \\
.04191\end{array}$ & $\begin{array}{l}57.60 \\
54.87 \\
52.28 \\
49.81 \\
47.46 \\
45.20 \\
43.05 \\
40.98 \\
38.99 \\
37.09\end{array}$ & $\begin{array}{l}.00232 \\
.00225 \\
.00219 \\
.00212 \\
.00205 \\
.00199 \\
.00193 \\
.00186 \\
.00180 \\
.00174\end{array}$ & $\begin{array}{l}250 . \\
255 . \\
260 . \\
265 . \\
270 . \\
275 . \\
280 . \\
295 . \\
290 . \\
295 .\end{array}$ & $\begin{array}{l}58.6756 \\
57.6943 \\
56.6881 \\
55.6557 \\
54.5952 \\
53.5052 \\
52.3841 \\
51.2306 \\
50.0435 \\
48.8217\end{array}$ & $\begin{array}{l}.05929 \\
.05738 \\
.05550 \\
.05366 \\
.05187 \\
.05018 \\
.04853 \\
.04673 \\
.04499 \\
.04331\end{array}$ & $\begin{array}{l}58.85 \\
56.14 \\
53.56 \\
51.12 \\
48.79 \\
46.57 \\
44.45 \\
42.42 \\
40.48 \\
38.62\end{array}$ & $\begin{array}{l}.00238 \\
.00231 \\
.00225 \\
.00219 \\
.00213 \\
.00207 \\
.00201 \\
.00195 \\
.00189 \\
.00183\end{array}$ \\
\hline $\begin{array}{l}300 . \\
310 . \\
320 . \\
330 . \\
340 . \\
350 . \\
360 . \\
370 . \\
380 . \\
390 .\end{array}$ & $\begin{array}{l}46.4347 \\
43.5667 \\
40.5343 \\
37.4139 \\
34.3443 \\
31.4797 \\
28.9239 \\
26.7086 \\
24.8145 \\
23.1995\end{array}$ & $\begin{array}{l}.04025 \\
.03713 \\
.03433 \\
.03187 \\
.02971 \\
.02782 \\
.02618 \\
.02478 \\
.02363 \\
.02269\end{array}$ & $\begin{array}{l}35.26 \\
31.82 \\
28.68 \\
25.91 \\
23.56 \\
21.67 \\
20.21 \\
19.11 \\
18.30 \\
17.70\end{array}$ & $\begin{array}{l}.00168 \\
.00157 \\
.00150 \\
.00147 \\
.00150 \\
.00158 \\
.00171 \\
.00187 \\
.00205 \\
.00225\end{array}$ & $\begin{array}{l}300 . \\
310 . \\
320 . \\
330 . \\
340 . \\
350 . \\
360 . \\
370 . \\
380 . \\
390 .\end{array}$ & $\begin{array}{l}47.5649 \\
44.9476 \\
42.2074 \\
39.3892 \\
36.5754 \\
33.8715 \\
31.3709 \\
29.1275 \\
27.1531 \\
25.4317\end{array}$ & $\begin{array}{l}.04169 \\
.03864 \\
.03589 \\
.03345 \\
.03131 \\
.02945 \\
.02782 \\
.02640 \\
.02520 \\
.02418\end{array}$ & $\begin{array}{l}36.85 \\
33.52 \\
30.50 \\
27.79 \\
25.44 \\
23.47 \\
21.87 \\
20.62 \\
19.63 \\
18.90\end{array}$ & $\begin{array}{l}.00178 \\
.00168 \\
.00161 \\
.00157 \\
.00157 \\
.00162 \\
.00171 \\
.00183 \\
.00198 \\
.00215\end{array}$ \\
\hline $\begin{array}{l}400 . \\
410 . \\
420 . \\
430 . \\
440 . \\
450 . \\
460 . \\
470 . \\
480 . \\
490 .\end{array}$ & $\begin{array}{l}21.8164 \\
20.6228 \\
19.5830 \\
18.6587 \\
17.8573 \\
17.1314 \\
16.4771 \\
15.8833 \\
15.3412 \\
14.8437\end{array}$ & $\begin{array}{l}.02196 \\
.02138 \\
.02094 \\
.02060 \\
.02035 \\
.02017 \\
.02004 \\
.011996 \\
.01992 \\
.01990\end{array}$ & $\begin{array}{l}17.26 \\
16.93 \\
16.70 \\
16.53 \\
16.42 \\
16.35 \\
16.32 \\
16.31 \\
16.32 \\
16.36\end{array}$ & $\begin{array}{l}.00246 \\
.00267 \\
.00290 \\
.00312 \\
.00334 \\
.00357 \\
.00380 \\
.00402 \\
.00425 \\
.00448\end{array}$ & $\begin{array}{l}400 . \\
410 . \\
420 . \\
430 . \\
440 . \\
450 . \\
460 . \\
470 . \\
480 . \\
490 .\end{array}$ & $\begin{array}{l}23.9341 \\
22.6276 \\
21.4818 \\
20.4700 \\
19.5701 \\
18.7642 \\
18.0375 \\
17.3781 \\
16.7765 \\
16.2248\end{array}$ & $\begin{array}{l}.02335 \\
.02267 \\
.02213 \\
.02170 \\
.02137 \\
.02111 \\
.02092 \\
.02078 \\
.02069 \\
.02063\end{array}$ & $\begin{array}{l}18.34 \\
17.92 \\
17.58 \\
17.34 \\
17.16 \\
17.04 \\
16.95 \\
16.90 \\
16.88 \\
16.88\end{array}$ & $\begin{array}{l}.00233 \\
.00252 \\
.00272 \\
.00292 \\
.00312 \\
.00333 \\
.00353 \\
.00374 \\
.00395 \\
.00416\end{array}$ \\
\hline $\begin{array}{l}500 . \\
510 . \\
520 . \\
530 . \\
540 .\end{array}$ & $\begin{array}{l}14.3849 \\
13.9501 \\
13.5651 \\
13.1964 \\
12.8518\end{array}$ & $\begin{array}{l}.01992 \\
.01995 \\
.02000 \\
.02007 \\
.02015\end{array}$ & $\begin{array}{l}16.40 \\
16.46 \\
16.53 \\
16.61 \\
16.70\end{array}$ & $\begin{array}{l}.00471 \\
.00494 \\
.00517 \\
.00540 \\
.00564\end{array}$ & $\begin{array}{l}500 . \\
510 . \\
520 . \\
530 . \\
540 .\end{array}$ & $\begin{array}{l}15.7164 \\
15.2460 \\
14.8090 \\
14.4017 \\
14.0208\end{array}$ & $\begin{array}{l}.02060 \\
.02059 \\
.02061 \\
.02065 \\
.02070\end{array}$ & $\begin{array}{l}16.90 \\
16.93 \\
16.98 \\
17.04 \\
17.11\end{array}$ & $\begin{array}{l}.00437 \\
.00458 \\
.00430 \\
.00501 \\
.00522\end{array}$ \\
\hline
\end{tabular}


Table 7. Transoort Properties of Oxygen. Isobars. Engr. Unlts.

?500. Psla Isobar

2800. DSia IsODar

\begin{tabular}{|c|c|c|c|c|c|c|c|c|c|}
\hline Temp. & $\begin{array}{l}\text { Density } \\
1 b / C t * * 3\end{array}$ & $\begin{array}{l}\text { Thermal } \\
\text { Cond. } \\
\text { Brulft.n.R }\end{array}$ & $\begin{array}{c}\text { Viscosity } \\
\text { ricro- } \\
\text { tbift.s }\end{array}$ & $\begin{array}{c}\text { Thermal } \\
\text { DIffusivity } \\
\text { ft*\#2/n }\end{array}$ & Teno. & $\begin{array}{l}\text { Jensity } \\
\text { Indet**3 }\end{array}$ & $\begin{array}{l}\text { Thermal } \\
\text { Cond. } \\
\text { arulftin.R }\end{array}$ & $\begin{array}{c}\text { viscosity } \\
\text { micro- } \\
\text { itites }\end{array}$ & $\begin{array}{c}\text { Thernal } \\
n 1 f \text { fusivity } \\
\text { ft*\#2In }\end{array}$ \\
\hline $\begin{array}{l}101.504 \\
105 . \\
110 . \\
115 . \\
120 . \\
125 . \\
130 . \\
135 . \\
140 . \\
145 .\end{array}$ & $\begin{array}{l}92.2447 \\
81.7374 \\
81.0170 \\
80.2990 \\
79.5842 \\
78.8584 \\
78.1514 \\
77.4325 \\
76.7109 \\
75.9842\end{array}$ & $\begin{array}{l}.12010 \\
.11827 \\
.11569 \\
.11328 \\
.11100 \\
.10891 \\
.10471 \\
.10467 \\
.10287 \\
.10070\end{array}$ & $\begin{array}{l}362.11 \\
344.95 \\
321.20 \\
290.58 \\
277.56 \\
257.96 \\
230.50 \\
223.11 \\
207.79 \\
193.76\end{array}$ & $\begin{array}{l}.00370 \\
.00367 \\
.00362 \\
.00350 \\
.00354 \\
.00352 \\
.00349 \\
.00346 \\
.00343 \\
.00340\end{array}$ & $\begin{array}{l}+101.731 \\
105 . \\
110 . \\
115 . \\
120 . \\
125 . \\
130 . \\
135 . \\
140 . \\
145 .\end{array}$ & $\begin{array}{l}92.2965 \\
81.8311 \\
91.1134 \\
80.3997 \\
79.4878 \\
78.9763 \\
79.2540 \\
77.5501 \\
74.8340 \\
76.1150\end{array}$ & $\begin{array}{l}.12039 \\
.111862 \\
.111305 \\
.11365 \\
.11138 \\
.10721 \\
.10712 \\
.10509 \\
.10312 \\
.10115\end{array}$ & $\begin{array}{l}344.75 \\
348.71 \\
324.63 \\
301.83 \\
280.47 \\
250.63 \\
242.31 \\
225.45 \\
220.99 \\
195.83\end{array}$ & $\begin{array}{l}.00370 \\
.00367 \\
00343 \\
.00330 \\
.00357 \\
.00354 \\
.00351 \\
.00348 \\
.00345 \\
.00347\end{array}$ \\
\hline $\begin{array}{l}150 . \\
155 . \\
160 . \\
165 . \\
170 . \\
175 . \\
180 . \\
185 . \\
190 . \\
195 .\end{array}$ & $\begin{array}{l}75.2578 \\
74.5255 \\
73.7887 \\
73.0471 \\
72.3003 \\
71.5477 \\
70.7889 \\
70.0234 \\
69.2505 \\
68.4596\end{array}$ & $\begin{array}{l}.09975 \\
.09582 \\
.09490 \\
.09298 \\
.09104 \\
.08914 \\
.08732 \\
.08530 \\
.08337 \\
.08144\end{array}$ & $\begin{array}{l}180.91 \\
169.16 \\
159.39 \\
148.54 \\
139.50 \\
131.21 \\
123.58 \\
113.56 \\
110.09 \\
104.10\end{array}$ & $\begin{array}{l}.00337 \\
.00334 \\
000271 \\
.00327 \\
.00324 \\
.00320 \\
.00314 \\
.00312 \\
.00307 \\
.00303\end{array}$ & $\begin{array}{l}150 . \\
155 . \\
160 . \\
155 . \\
170 . \\
175 . \\
180 . \\
185 . \\
190 . \\
195 .\end{array}$ & $\begin{array}{l}75.3728 \\
74.6659 \\
73.9371 \\
73.2028 \\
72.4637 \\
71.7194 \\
70.9595 \\
70.2133 \\
59.4505 \\
69.6905\end{array}$ & $\begin{array}{l}.07922 \\
.09730 \\
.09540 \\
.09350 \\
.07160 \\
.08970 \\
.08780 \\
.08590 \\
.08399 \\
.09208\end{array}$ & $\begin{array}{l}132.86 \\
171.20 \\
150.15 \\
153.71 \\
141.10 \\
132.74 \\
125.06 \\
117.98 \\
111.45 \\
105.44\end{array}$ & $\begin{array}{l}.00339 \\
.00333 \\
.00332 \\
.00329 \\
.00326 \\
.00322 \\
.00318 \\
.00314 \\
.00310 \\
.00303\end{array}$ \\
\hline $\begin{array}{l}200 . \\
2050^{\circ} \\
210 . \\
2150^{\circ} \\
220 . \\
225 . \\
230 . \\
2350^{\circ} \\
240 . \\
245 .\end{array}$ & $\begin{array}{l}67.5800 \\
66.5809 \\
66.0715 \\
65.2510 \\
64.4293 \\
63.5725 \\
62.7125 \\
61.8373 \\
60.9456 \\
60.3364\end{array}$ & $\begin{array}{l}.07951 \\
.07758 \\
.07564 \\
.07370 \\
.07177 \\
.06993 \\
.07790 \\
.06508 \\
.06407 \\
.01216\end{array}$ & $\begin{array}{l}98.57 \\
93.43 \\
88.64 \\
84.21 \\
80.04 \\
73.19 \\
72.55 \\
59.14 \\
65.73 \\
62.91\end{array}$ & $\begin{array}{l}.00209 \\
.00793 \\
.00289 \\
.00283 \\
.00778 \\
.00272 \\
.00267 \\
.00261 \\
.00255 \\
.00249\end{array}$ & $\begin{array}{l}200 . \\
205 . \\
210 . \\
215 . \\
220 . \\
225 . \\
230 . \\
235 . \\
240 . \\
245 .\end{array}$ & $\begin{array}{l}67.9025 \\
67.1160 \\
66.3201 \\
55.5143 \\
64.5975 \\
63.8691 \\
63.0280 \\
62.1735 \\
61.3044 \\
60.4200\end{array}$ & $\begin{array}{l}.08017 \\
.07826 \\
.07635 \\
.07444 \\
.07253 \\
.07062 \\
.06873 \\
.06683 \\
.06495 \\
.06308\end{array}$ & $\begin{array}{l}77.97 \\
94.70 \\
89.70 \\
95.43 \\
81.27 \\
77.38 \\
73.73 \\
70.31 \\
57.10 \\
54.08\end{array}$ & $\begin{array}{l}.00301 \\
.00275 \\
.00292 \\
.00297 \\
.00281 \\
.00274 \\
.00271 \\
.00255 \\
.00260 \\
.00254\end{array}$ \\
\hline $\begin{array}{l}250 . \\
255 . \\
260 . \\
265 . \\
270 . \\
275 . \\
280 . \\
285 . \\
790 . \\
295 .\end{array}$ & $\begin{array}{l}59.1085 \\
58.1405 \\
57.1914 \\
54.1090 \\
55.1950 \\
54.1455 \\
53.0805 \\
51.9802 \\
50.8710 \\
49.7255\end{array}$ & $\begin{array}{l}.060 ? 8 \\
.05841 \\
.05457 \\
.05476 \\
.05301 \\
.05134 \\
.04971 \\
.04796 \\
.04626 \\
.04462\end{array}$ & $\begin{array}{l}60.06 \\
57.35 \\
54.78 \\
52.36 \\
50.06 \\
47.86 \\
45.76 \\
43.77 \\
41.86 \\
40.04\end{array}$ & $\begin{array}{l}.00243 \\
.00237 \\
.00231 \\
.00225 \\
.00219 \\
.00214 \\
.00208 \\
.00203 \\
.00197 \\
.00191\end{array}$ & $\begin{array}{l}250 . \\
255 . \\
260 . \\
265 . \\
270 . \\
275 . \\
280 . \\
285 . \\
290 . \\
295 .\end{array}$ & $\begin{array}{l}59.5191 \\
58.6010 \\
57.6646 \\
56.7091 \\
55.7336 \\
54.7376 \\
53.7203 \\
52.5812 \\
51.6203 \\
50.5373\end{array}$ & $\begin{array}{l}.06123 \\
.05939 \\
.05759 \\
.05581 \\
.05409 \\
.05245 \\
.05083 \\
.04913 \\
.04747 \\
.04587\end{array}$ & $\begin{array}{l}61.22 \\
58.53 \\
55.98 \\
53.56 \\
51.27 \\
49.08 \\
47.01 \\
45.03 \\
43.15 \\
41.36\end{array}$ & $\begin{array}{l}.00248 \\
.00242 \\
.00237 \\
.00231 \\
.00226 \\
.00220 \\
.00215 \\
.00210 \\
.00254 \\
.00109\end{array}$ \\
\hline $\begin{array}{l}300 . \\
310 . \\
320 . \\
330 . \\
340 . \\
350 . \\
360 . \\
370 . \\
380 . \\
390 .\end{array}$ & $\begin{array}{l}48.5527 \\
43.1277 \\
43.5088 \\
41.0260 \\
38.4318 \\
35.2975 \\
33.4953 \\
31.2835 \\
29.2664 \\
27.5075\end{array}$ & $\begin{array}{l}.04304 \\
.04006 \\
.03734 \\
.03492 \\
.03278 \\
.03092 \\
.02529 \\
.02787 \\
.02364 \\
.02558\end{array}$ & $\begin{array}{l}38.30 \\
35.05 \\
32.11 \\
29.46 \\
27.13 \\
25.13 \\
23.45 \\
22.08 \\
20.99 \\
20.12\end{array}$ & $\begin{array}{l}.00186 \\
.00177 \\
.00170 \\
.00166 \\
.00165 \\
.00167 \\
.00173 \\
.00183 \\
.00195 \\
.00209\end{array}$ & $\begin{array}{l}300 . \\
310 . \\
320 . \\
330 . \\
340 . \\
350 . \\
360 . \\
370 . \\
380 . \\
390 .\end{array}$ & $\begin{array}{l}49.4325 \\
47.1607 \\
44.8157 \\
42.4196 \\
40.0083 \\
37.5313 \\
35.3435 \\
33.1932 \\
31.2125 \\
29.4144\end{array}$ & $\begin{array}{l}.04432 \\
.04139 \\
.03871 \\
.03630 \\
.03416 \\
.03229 \\
.03065 \\
.02922 \\
.02797 \\
.02688\end{array}$ & $\begin{array}{l}39.65 \\
36.46 \\
33.57 \\
30.98 \\
28.67 \\
26.53 \\
24.92 \\
23.48 \\
22.29 \\
21.32\end{array}$ & $\begin{array}{l}.00194 \\
.00186 \\
.00179 \\
.00174 \\
.00177 \\
.00173 \\
.00177 \\
.00184 \\
.00194 \\
.00206\end{array}$ \\
\hline $\begin{array}{l}400 . \\
410 . \\
420 . \\
430 . \\
440 . \\
450 . \\
460 . \\
470 . \\
480 . \\
490 .\end{array}$ & $\begin{array}{l}25.9331 \\
24.5420 \\
23.3104 \\
22.2159 \\
21.2380 \\
20.3597 \\
19.5663 \\
19.9457 \\
18.1879 \\
17.5843\end{array}$ & $\begin{array}{l}.02468 \\
.02393 \\
.02331 \\
.02280 \\
.02240 \\
.02207 \\
.02187 \\
.02152 \\
.02147 \\
.02137\end{array}$ & $\begin{array}{l}19.44 \\
18.91 \\
18.50 \\
18.18 \\
17.94 \\
17.75 \\
17.69 \\
17.52 \\
17.46 \\
17.42\end{array}$ & $\begin{array}{l}.00224 \\
.00241 \\
.00258 \\
.00277 \\
.00295 \\
.00314 \\
.00333 \\
.00352 \\
.00371 \\
.00390\end{array}$ & $\begin{array}{l}400 . \\
410 . \\
420 . \\
430 . \\
440 . \\
450 . \\
460 . \\
470 . \\
480 . \\
490 .\end{array}$ & $\begin{array}{l}27.7969 \\
26.3484 \\
25.0525 \\
23.8915 \\
22.8482 \\
21.9070 \\
21.0542 \\
20.2779 \\
19.5681 \\
18.9165\end{array}$ & $\begin{array}{l}.02594 \\
.02514 \\
.02246 \\
.02389 \\
.02342 \\
.02303 \\
.02272 \\
.02247 \\
.02227 \\
.02212\end{array}$ & $\begin{array}{l}20.55 \\
19.93 \\
19.43 \\
19.04 \\
18.73 \\
18.49 \\
18.30 \\
18.16 \\
18.06 \\
17.99\end{array}$ & $\begin{array}{l}.00219 \\
.00234 \\
.00249 \\
.00265 \\
.00282 \\
.00299 \\
.00316 \\
.00334 \\
.00352 \\
.00370\end{array}$ \\
\hline $\begin{array}{l}500 . \\
510 . \\
520 . \\
530 . \\
540 .\end{array}$ & $\begin{array}{l}17.0283 \\
16.5140 \\
16.0363 \\
15.5913 \\
15.1754\end{array}$ & $\begin{array}{l}.02129 \\
.02125 \\
.02123 \\
.02124 \\
.02126\end{array}$ & $\begin{array}{l}17.41 \\
17.42 \\
17.44 \\
17.48 \\
17.52\end{array}$ & $\begin{array}{l}.00410 \\
.00429 \\
.00449 \\
.00459 \\
.00499\end{array}$ & $\begin{array}{l}500 . \\
510 . \\
520 . \\
530 . \\
540 .\end{array}$ & $\begin{array}{l}16.3156 \\
17.7596 \\
17.2432 \\
16.7621 \\
16.3124\end{array}$ & $\begin{array}{l}.02200 \\
.02192 \\
.02187 \\
.02184 \\
.02183\end{array}$ & $\begin{array}{l}17.94 \\
17.92 \\
17.92 \\
17.93 \\
17.96\end{array}$ & $\begin{array}{l}.00388 \\
.00406 \\
.00424 \\
.00442 \\
.00460\end{array}$ \\
\hline
\end{tabular}

* Two Phase Roundary 
Table 7. Transport Propertles of Cxygen. Isobars, Engr. Unlts.

3000. Dsla Isobar

Temp. Density Thermel viscosity Thermal

R Infet*3 BTulfting iblftes olfusivit

3200. DSla lsobar

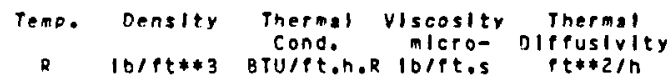

\begin{tabular}{|c|c|c|c|c|c|c|c|c|c|}
\hline $\begin{array}{l}102.058 \\
105 . \\
110 . \\
115 . \\
120 . \\
125 . \\
130 . \\
235 . \\
140 . \\
145 .\end{array}$ & $\begin{array}{l}82.3481 \\
81.9240 \\
81.2090 \\
80.4986 \\
79.7905 \\
79.0832 \\
78.3755 \\
77.5365 \\
76.9556 \\
76.2423\end{array}$ & $\begin{array}{l}.12058 \\
.11897 \\
.11641 \\
.11401 \\
.11175 \\
.10960 \\
.10752 \\
.10550 \\
.10353 \\
.10159\end{array}$ & $\begin{array}{l}367.36 \\
352.49 \\
328.08 \\
304.90 \\
283.39 \\
263.33 \\
244.82 \\
227.90 \\
212.19 \\
197.99\end{array}$ & $\begin{array}{l}.00371 \\
.00368 \\
.00364 \\
.00361 \\
.00358 \\
.00355 \\
.00352 \\
.00349 \\
.00346 \\
.00343\end{array}$ & $\begin{array}{l}+102.334 \\
1050^{\circ} \\
110 . \\
115 . \\
120 . \\
125 . \\
130 . \\
135 . \\
140 . \\
145 .\end{array}$ & $\begin{array}{l}82.3094 \\
82.0163 \\
81.3039 \\
80.5966 \\
79.8923 \\
79.1890 \\
78.4858 \\
77.7816 \\
77.0759 \\
76.3680\end{array}$ & $\begin{array}{l}.12077 \\
.11932 \\
.11676 \\
.11438 \\
.11213 \\
.10998 \\
.10792 \\
.10592 \\
.10396 \\
.10204\end{array}$ & $\begin{array}{l}369.96 \\
355.29 \\
331.54 \\
308.16 \\
286.31 \\
265.04 \\
247.33 \\
239.14 \\
214.38 \\
199.96\end{array}$ & $\begin{array}{l}.00371 \\
.00369 \\
.00365 \\
.00362 \\
.00359 \\
.00356 \\
.00353 \\
.00350 \\
.00347 \\
.00345\end{array}$ \\
\hline $\begin{array}{l}150 . \\
155 . \\
160 . \\
165 . \\
170 \\
175 . \\
180 . \\
185 . \\
190 . \\
195 .\end{array}$ & $\begin{array}{l}75.5260 \\
74.8065 \\
74.0833 \\
73.3561 \\
72.6245 \\
71.8881 \\
71.1466 \\
70.3095 \\
69.5464 \\
68.8866\end{array}$ & $\begin{array}{l}.09968 \\
.09778 \\
.09589 \\
.09401 \\
.09213 \\
.00025 \\
.09877 \\
.08649 \\
.08460 \\
.08272\end{array}$ & $\begin{array}{l}184.81 \\
172.85 \\
161.80 \\
151.89 \\
142.70 \\
134.27 \\
126.52 \\
119.40 \\
112.83 \\
106.77\end{array}$ & $\begin{array}{l}.00340 \\
.00337 \\
.00334 \\
.00331 \\
.00328 \\
.00324 \\
.00320 \\
.00316 \\
.00312 \\
.00308\end{array}$ & $\begin{array}{l}150 . \\
155 . \\
160 . \\
155 . \\
170 . \\
175 . \\
180 . \\
185 . \\
170 . \\
195 .\end{array}$ & $\begin{array}{l}75.6575 \\
74.9441 \\
74.2274 \\
73.5070 \\
72.7827 \\
72.0540 \\
71.3207 \\
70.5822 \\
69.8382 \\
69.0883\end{array}$ & $\begin{array}{l}.10014 \\
.09826 \\
.09639 \\
.09452 \\
.09266 \\
.09080 \\
.08893 \\
.08707 \\
.08520 \\
.08334\end{array}$ & $\begin{array}{l}136.76 \\
174.59 \\
163.65 \\
153.54 \\
144.28 \\
135.79 \\
127.98 \\
120.80 \\
114.19 \\
108.08\end{array}$ & $\begin{array}{l}.00342 \\
.00339 \\
.00336 \\
.00333 \\
.00329 \\
.00326 \\
.00322 \\
.00314 \\
.00315 \\
.00311\end{array}$ \\
\hline $\begin{array}{l}200 . \\
205 . \\
210 . \\
2150 \\
220 . \\
2250 \\
230 . \\
235 . \\
240 . \\
2450\end{array}$ & $\begin{array}{l}68.1197 \\
67.3450 \\
66.5320 \\
65.7699 \\
64.9581 \\
64.1558 \\
63.3322 \\
62.4967 \\
61.6494 \\
60.7864\end{array}$ & $\begin{array}{l}.08 C 93 \\
.07894 \\
.07705 \\
.07516 \\
.07328 \\
.07140 \\
.06952 \\
.06766 \\
.06581 \\
.06797\end{array}$ & $\begin{array}{r}101.16 \\
95.96 \\
91.13 \\
86.64 \\
82.45 \\
78.55 \\
74.89 \\
71.46 \\
68.24 \\
65.21\end{array}$ & $\begin{array}{l}.00304 \\
.00299 \\
.00295 \\
.00290 \\
.00285 \\
.00280 \\
.00275 \\
.00269 \\
.00264 \\
.00259\end{array}$ & $\begin{array}{l}200 . \\
205 . \\
210 . \\
215 . \\
220 . \\
225 . \\
230 . \\
235 . \\
240 . \\
245 .\end{array}$ & $\begin{array}{l}58.3319 \\
67.5685 \\
66.7975 \\
66.0184 \\
65.2306 \\
64.4334 \\
33.6261 \\
62.8082 \\
61.9789 \\
61.1376\end{array}$ & $\begin{array}{l}.08147 \\
.07960 \\
.07773 \\
.07587 \\
.07401 \\
.07215 \\
.07031 \\
.06847 \\
.06564 \\
.08483\end{array}$ & $\begin{array}{r}102.43 \\
97.20 \\
92.34 \\
87.83 \\
83.62 \\
79.70 \\
76.02 \\
72.59 \\
69.35 \\
66.32\end{array}$ & $\begin{array}{l}.00307 \\
.00302 \\
.00208 \\
.00293 \\
.00286 \\
.00283 \\
.00278 \\
.00273 \\
.00268 \\
.00263\end{array}$ \\
\hline $\begin{array}{l}250 . \\
255 . \\
260 . \\
265 . \\
270 . \\
275 . \\
280 . \\
285 . \\
290 . \\
295 .\end{array}$ & $\begin{array}{l}59.9102 \\
59.0188 \\
58.1116 \\
57.1880 \\
56.2473 \\
55.2890 \\
54.3129 \\
53.3196 \\
52.3062 \\
51.2757\end{array}$ & $\begin{array}{l}.06215 \\
.06034 \\
.05457 \\
.05683 \\
.05513 \\
.05351 \\
.05191 \\
.05024 \\
.04842 \\
.04705\end{array}$ & $\begin{array}{l}62.35 \\
59.67 \\
57.12 \\
54.71 \\
52.43 \\
50.26 \\
48.20 \\
44.24 \\
44.37 \\
42.60\end{array}$ & $\begin{array}{l}.00253 \\
.00248 \\
.00242 \\
.00237 \\
.00231 \\
.00226 \\
.00221 \\
.00216 \\
.00211 \\
.00206\end{array}$ & $\begin{array}{l}250 . \\
255 . \\
260 . \\
265 . \\
270 . \\
275 . \\
280 . \\
285 . \\
270 . \\
205 .\end{array}$ & $\begin{array}{l}50.2837 \\
59.4166 \\
58.5357 \\
57.6405 \\
56.7307 \\
55.8058 \\
54.8657 \\
53.9103 \\
52.9397 \\
51.9541\end{array}$ & $\begin{array}{l}.05304 \\
.06126 \\
.05951 \\
.05780 \\
.05613 \\
.05453 \\
.05295 \\
.05131 \\
.04972 \\
.04818\end{array}$ & $\begin{array}{l}53.47 \\
60.77 \\
58.23 \\
55.82 \\
53.54 \\
51.38 \\
49.33 \\
47.39 \\
45.53 \\
43.77\end{array}$ & $\begin{array}{l}.0025 B \\
.00252 \\
.00247 \\
.00242 \\
.00237 \\
.00232 \\
.00227 \\
.00222 \\
.00217 \\
.00217\end{array}$ \\
\hline $\begin{array}{l}300 . \\
310 . \\
370 . \\
330 . \\
340 . \\
350 . \\
360 . \\
370 . \\
380 . \\
390 .\end{array}$ & $\begin{array}{l}5 C .2276 \\
48.0814 \\
45.8772 \\
43.5323 \\
41.3731 \\
39.1750 \\
36.9503 \\
34.9956 \\
32.9447 \\
31.1545\end{array}$ & $\begin{array}{l}.04553 \\
.04265 \\
.04000 \\
.03760 \\
.03547 \\
.03358 \\
.03193 \\
.03048 \\
.02921 \\
.02810\end{array}$ & $\begin{array}{l}40.91 \\
37.77 \\
34.92 \\
32.36 \\
30.07 \\
28.05 \\
26.30 \\
24.00 \\
23.54 \\
27.50\end{array}$ & $\begin{array}{l}.00202 \\
.00194 \\
.00187 \\
.00182 \\
.00179 \\
.00179 \\
.00182 \\
.00187 \\
.00195 \\
.00205\end{array}$ & $\begin{array}{l}300 . \\
310 . \\
320 . \\
330 . \\
340 . \\
350 . \\
360 . \\
370 . \\
380 . \\
390 .\end{array}$ & $\begin{array}{l}50.9540 \\
48.9132 \\
46.8200 \\
44.7063 \\
42.5745 \\
40.4572 \\
39.3958 \\
35.3921 \\
34.5033 \\
32.7394\end{array}$ & $\begin{array}{l}.04669 \\
.04385 \\
.04123 \\
.03885 \\
.03671 \\
.03481 \\
.03314 \\
.03167 \\
.03038 \\
.02925\end{array}$ & $\begin{array}{l}42.10 \\
38.77 \\
35.18 \\
33.64 \\
31.37 \\
29.35 \\
27.59 \\
25.05 \\
24.74 \\
23.54\end{array}$ & $\begin{array}{l}.00200 \\
.00231 \\
.00184 \\
.00189 \\
.00184 \\
.00125 \\
.00187 \\
.00171 \\
.00177 \\
.00205\end{array}$ \\
\hline $\begin{array}{l}400 . \\
410 . \\
420 .\end{array}$ & $\begin{array}{l}29.5203 \\
28.0381 \\
26.6980\end{array}$ & $\begin{array}{l}.02713 \\
.02629 \\
.02557\end{array}$ & $\begin{array}{l}21.64 \\
20.94 \\
20.37\end{array}$ & $\begin{array}{l}.00216 \\
.00229 \\
.00243\end{array}$ & $\begin{array}{l}400 . \\
410 \\
420\end{array}$ & $\begin{array}{l}31.1070 \\
29.6101 \\
28.2429\end{array}$ & $\begin{array}{l}.02 \pi 26 \\
.02739 \\
.02663\end{array}$ & $\begin{array}{l}22.71 \\
21.94 \\
21.30\end{array}$ & $\begin{array}{l}.00215 \\
.00225 \\
.02234\end{array}$ \\
\hline $\begin{array}{l}430 . \\
440 . \\
450 . \\
460 . \\
470 . \\
480 . \\
490 .\end{array}$ & $\begin{array}{l}25.4870 \\
24.3913 \\
23.3974 \\
22.4935 \\
21.6681 \\
20.9117 \\
20.2160\end{array}$ & $\begin{array}{l}.02495 \\
.02442 \\
.02398 \\
.02362 \\
.02332 \\
.02307 \\
.02288\end{array}$ & $\begin{array}{l}19.91 \\
19.54 \\
19.24 \\
19.01 \\
18.92 \\
18.58 \\
18.57\end{array}$ & $\begin{array}{l}.00257 \\
.00272 \\
.00288 \\
.00304 \\
.00320 \\
.00335 \\
.00353\end{array}$ & $\begin{array}{l}430 . \\
440 . \\
450 . \\
450 . \\
470 . \\
480 . \\
490 .\end{array}$ & $\begin{array}{l}26.9967 \\
25.8612 \\
24.8254 \\
23.8787 \\
23.0111 \\
23.2138 \\
21.4788\end{array}$ & $\begin{array}{l}.02597 \\
.02540 \\
.02492 \\
.02451 \\
.02417 \\
.02388 \\
.02344\end{array}$ & $\begin{array}{l}20.78 \\
20.35 \\
20.00 \\
19.72 \\
19.49 \\
19.31 \\
19.16\end{array}$ & $\begin{array}{l}.00252 \\
.00235 \\
.03279 \\
.00294 \\
.00309 \\
.00324 \\
.00334\end{array}$ \\
\hline $\begin{array}{l}5 c c . \\
510 . \\
520 . \\
530 \\
540 .\end{array}$ & $\begin{array}{l}10.5738 \\
18.9790 \\
18.4267 \\
17.9109 \\
17.4291\end{array}$ & $\begin{array}{l}.02272 \\
.02260 \\
.02252 \\
.02245 \\
.02242\end{array}$ & $\begin{array}{l}18.49 \\
18.44 \\
18.41 \\
18.40 \\
18.40\end{array}$ & $\begin{array}{l}.00349 \\
.00380 \\
.00403 \\
.00420 \\
.00437\end{array}$ & $\begin{array}{l}500 . \\
510 . \\
520 . \\
530 . \\
540 .\end{array}$ & $\begin{array}{l}20.7991 \\
20.1587 \\
17.5822 \\
10.0350 \\
18.5231\end{array}$ & $\begin{array}{l}.02345 \\
.02329 \\
.02317 \\
.02307 \\
.02301\end{array}$ & $\begin{array}{l}19.05 \\
18.77 \\
18.91 \\
18.88 \\
18.85\end{array}$ & $\begin{array}{l}.00354 \\
.00370 \\
.00334 \\
.00431 \\
.00417\end{array}$ \\
\hline
\end{tabular}


Tohle 7. Transport Propertles of Oxyen, Isobers, Engr. Unlts.

3400. PsI. Isober

3600. Dsia Isobar

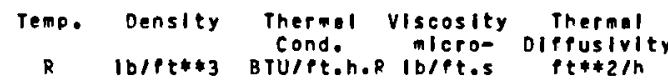

\begin{tabular}{|c|c|c|c|c|c|c|c|c|c|}
\hline $\begin{array}{l}102.610 \\
105 . \\
110 . \\
115 . \\
120 . \\
125 . \\
130 . \\
135 . \\
140 . \\
145 .\end{array}$ & $\begin{array}{l}82.4503 \\
62.1079 \\
81.3980 \\
80.6939 \\
79.0931 \\
79.2938 \\
78.5950 \\
77.8955 \\
77.1947 \\
76.4922\end{array}$ & $\begin{array}{l}.12096 \\
.11967 \\
.11712 \\
.11474 \\
.111250 \\
.11037 \\
.10831 \\
.10633 \\
.10438 \\
.10248\end{array}$ & $\begin{array}{l}372.53 \\
360.10 \\
335.01 \\
311.34 \\
289.23 \\
268.74 \\
249.85 \\
232.49 \\
216.58 \\
202.02\end{array}$ & $\begin{array}{l}.00372 \\
.00370 \\
.00366 \\
.00363 \\
.00360 \\
.00357 \\
.00354 \\
.00352 \\
.00349 \\
.00346\end{array}$ & $\begin{array}{l}* 102.884 \\
105 . \\
110 . \\
115 . \\
120 . \\
125 . \\
130 . \\
135 . \\
140 . \\
145 .\end{array}$ & $\begin{array}{l}82.5010 \\
82.1988 \\
81.4914 \\
80.7903 \\
80.0930 \\
79.3977 \\
78.7030 \\
78.0081 \\
77.3123 \\
76.6149\end{array}$ & $\begin{array}{l}.12115 \\
.12001 \\
.11747 \\
.11510 \\
.11287 \\
.11075 \\
.10871 \\
.10673 \\
.10480 \\
.10291\end{array}$ & $\begin{array}{l}375.08 \\
363.93 \\
330.48 \\
314.52 \\
292.16 \\
271.45 \\
252.37 \\
234.83 \\
218.77 \\
204.08\end{array}$ & $\begin{array}{l}.00372 \\
.00370 \\
.00367 \\
.00364 \\
.00361 \\
.00358 \\
.00355 \\
.00353 \\
.00350 \\
.00348\end{array}$ \\
\hline $\begin{array}{l}150 . \\
155 . \\
160 . \\
165 . \\
170 . \\
175 . \\
180 . \\
185 . \\
190 . \\
195 .\end{array}$ & $\begin{array}{l}75.7873 \\
75.0799 \\
74.3694 \\
73.6557 \\
72.9384 \\
72.2171 \\
71.4916 \\
70.7615 \\
70.0263 \\
69.2858\end{array}$ & $\begin{array}{l}.10054 \\
.09873 \\
.09687 \\
.09502 \\
.09318 \\
.09133 \\
.08949 \\
.08764 \\
.08580 \\
.08395\end{array}$ & $\begin{array}{l}188.70 \\
176.53 \\
165.39 \\
155.20 \\
145.86 \\
137.30 \\
129.43 \\
122.20 \\
115.53 \\
109.38\end{array}$ & $\begin{array}{l}.00343 \\
.00341 \\
.00338 \\
.00335 \\
.00331 \\
.00328 \\
.00325 \\
.00321 \\
.00317 \\
.00313\end{array}$ & $\begin{array}{l}150 . \\
155 . \\
160 . \\
165 . \\
170 . \\
175 . \\
180 . \\
185 . \\
190 . \\
195 .\end{array}$ & $\begin{array}{l}75.9155 \\
75.2139 \\
74.5095 \\
73.8022 \\
73.0917 \\
72.3776 \\
71.6596 \\
70.9375 \\
70.2108 \\
69.4793\end{array}$ & $\begin{array}{l}.10104 \\
.09919 \\
.09736 \\
.09552 \\
.09369 \\
.09187 \\
.09004 \\
.08821 \\
.08638 \\
.08455\end{array}$ & $\begin{array}{l}190.64 \\
178.36 \\
167.13 \\
136.85 \\
167.44 \\
138.80 \\
130.88 \\
123.59 \\
116.87 \\
110.67\end{array}$ & $\begin{array}{l}.00345 \\
.00342 \\
.00339 \\
.00336 \\
.00333 \\
.00330 \\
.00327 \\
.00323 \\
.00319 \\
.00316\end{array}$ \\
\hline $\begin{array}{l}200 . \\
205 . \\
210 . \\
215 . \\
220 . \\
225 . \\
230 . \\
235 . \\
240 . \\
245 .\end{array}$ & $\begin{array}{l}68.5394 \\
67.7867 \\
67.0272 \\
66.2603 \\
65.4856 \\
64.7025 \\
63.9104 \\
63.1088 \\
62.2972 \\
61.4749\end{array}$ & $\begin{array}{l}.08210 \\
.08025 \\
.07841 \\
.07656 \\
.07472 \\
.07289 \\
.07107 \\
.06926 \\
.06745 \\
.06567\end{array}$ & $\begin{array}{r}103.70 \\
98.43 \\
93.54 \\
89.00 \\
84.77 \\
80.83 \\
77.14 \\
73.69 \\
70.45 \\
67.41\end{array}$ & $\begin{array}{l}.00309 \\
.00305 \\
.00301 \\
.00296 \\
.00291 \\
.00287 \\
.00282 \\
.00277 \\
.00272 \\
.00267\end{array}$ & $\begin{array}{l}200 . \\
205 \\
210 \\
215 \\
220 \\
225 \\
230 \\
235 \\
240 \\
245\end{array}$ & $\begin{array}{l}68.7424 \\
67.9999 \\
67.2512 \\
66.4960 \\
55.7336 \\
64.9638 \\
64.1859 \\
63.3996 \\
62.6043 \\
61.7996\end{array}$ & $\begin{array}{l}.08272 \\
.08089 \\
.07907 \\
.07725 \\
.07543 \\
.07362 \\
.07182 \\
.07002 \\
.06825 \\
.06648\end{array}$ & $\begin{array}{r}104.95 \\
99.65 \\
94.73 \\
90.16 \\
85.91 \\
81.94 \\
78.24 \\
74.77 \\
71.52 \\
68.47\end{array}$ & $\begin{array}{l}.00312 \\
.00308 \\
.00303 \\
.00299 \\
.00295 \\
.00290 \\
.00285 \\
.00281 \\
.00276 \\
.00271\end{array}$ \\
\hline $\begin{array}{l}250 . \\
255 . \\
260 . \\
265 . \\
270 . \\
275 . \\
280 . \\
285 . \\
290 . \\
295 .\end{array}$ & $\begin{array}{l}60.0415 \\
59.7965 \\
58.9394 \\
58.0699 \\
37.1877 \\
56.2925 \\
35.3844 \\
54.4632 \\
53.5291 \\
52.5825\end{array}$ & $\begin{array}{l}.06390 \\
.06213 \\
.06043 \\
.05874 \\
.05710 \\
.05551 \\
.03393 \\
.05234 \\
.05078 \\
.04926\end{array}$ & $\begin{array}{l}64.55 \\
61.85 \\
39.31 \\
56.90 \\
54.63 \\
52.47 \\
50.43 \\
48.49 \\
46.64 \\
44.89\end{array}$ & $\begin{array}{l}.00262 \\
.00257 \\
.00252 \\
.00247 \\
.00242 \\
.00237 \\
.00233 \\
.00228 \\
.00223 \\
.00219\end{array}$ & $\begin{array}{l}250 . \\
255 . \\
260 . \\
265 . \\
270 . \\
275 . \\
280 . \\
285 . \\
290 . \\
295 .\end{array}$ & $\begin{array}{l}60.9850 \\
60.1602 \\
59.3249 \\
58.4787 \\
57.6214 \\
56.7530 \\
55.8733 \\
54.9825 \\
54.0808 \\
53.1684\end{array}$ & $\begin{array}{l}.06474 \\
.06302 \\
.06132 \\
.05965 \\
.05803 \\
.05646 \\
.05492 \\
.05334 \\
.05180 \\
.05031\end{array}$ & $\begin{array}{l}65.60 \\
62.90 \\
60.35 \\
57.95 \\
55.67 \\
53.52 \\
51.48 \\
49.54 \\
47.71 \\
45.96\end{array}$ & $\begin{array}{l}.00266 \\
.00261 \\
.00256 \\
.00252 \\
.00247 \\
.00242 \\
.00238 \\
.00233 \\
.00229 \\
.00225\end{array}$ \\
\hline $\begin{array}{l}300 . \\
310 . \\
320 . \\
330 . \\
340 . \\
350 . \\
360 . \\
370 . \\
360 . \\
390 .\end{array}$ & $\begin{array}{l}51.6238 \\
49.6731 \\
47.6848 \\
45.6706 \\
43.6469 \\
41.6344 \\
39.6575 \\
37.7416 \\
35.9100 \\
34.1809\end{array}$ & $\begin{array}{l}.04779 \\
.04500 \\
.04241 \\
.04004 \\
.03790 \\
.03590 \\
.03430 \\
.03281 \\
.03150 \\
.03035\end{array}$ & $\begin{array}{l}43.23 \\
40.14 \\
37.35 \\
34.84 \\
32.58 \\
30.57 \\
28.79 \\
27.23 \\
25.88 \\
24.73\end{array}$ & $\begin{array}{l}.00215 \\
.00207 \\
.00201 \\
.00196 \\
.00193 \\
.00192 \\
.00192 \\
.00195 \\
.00200 \\
.00207\end{array}$ & $\begin{array}{l}300 . \\
310 . \\
320 . \\
330 . \\
340 . \\
350 . \\
360 . \\
370 . \\
380 . \\
390^{\circ} .\end{array}$ & $\begin{array}{l}52.2460 \\
50.3734 \\
48.4701 \\
46.5464 \\
44.6155 \\
42.6942 \\
40.8019 \\
38.9591 \\
37.1856 \\
35.4976\end{array}$ & $\begin{array}{l}.04886 \\
.04610 \\
.04353 \\
.04118 \\
.03904 \\
.03713 \\
.03542 \\
.03391 \\
.03258 \\
.03140\end{array}$ & $\begin{array}{l}44.31 \\
41.24 \\
38.47 \\
35.97 \\
33.72 \\
31.71 \\
29.92 \\
28.03 \\
26.97 \\
25.78\end{array}$ & $\begin{array}{l}.00221 \\
.00214 \\
.00207 \\
.00202 \\
.00199 \\
.00197 \\
.00198 \\
.00200 \\
.00204 \\
.00209\end{array}$ \\
\hline $\begin{array}{l}400 . \\
410 . \\
420 . \\
430 . \\
440 . \\
450 . \\
460 . \\
470 . \\
480 . \\
490 .\end{array}$ & $\begin{array}{l}32.5654 \\
31.0681 \\
29.6877 \\
28.4193 \\
27.2553 \\
26.11872 \\
25.2061 \\
24.3034 \\
23.4710 \\
22.7016\end{array}$ & $\begin{array}{l}.02933 \\
.02844 \\
.02765 \\
.02696 \\
.02636 \\
.02584 \\
.02539 \\
.02500 \\
.02468 \\
.02440\end{array}$ & $\begin{array}{l}23.75 \\
22.92 \\
22.23 \\
21.65 \\
21.16 \\
20.76 \\
20.43 \\
20.16 \\
19.94 \\
19.76\end{array}$ & $\begin{array}{l}.00215 \\
.00225 \\
.00236 \\
.00248 \\
.00260 \\
.00273 \\
.00286 \\
.00300 \\
.00314 \\
.00328\end{array}$ & $\begin{array}{l}400 . \\
410 . \\
420 . \\
430 . \\
440 . \\
450 . \\
460 . \\
470 . \\
480 . \\
490 .\end{array}$ & $\begin{array}{l}33.9067 \\
32.4191 \\
31.0364 \\
29.7561 \\
28.5734 \\
27.4817 \\
26.4739 \\
25.5427 \\
24.6810 \\
23.8821\end{array}$ & $\begin{array}{l}.03036 \\
.02944 \\
.02863 \\
.02791 \\
.02728 \\
.02673 \\
.02625 \\
.02583 \\
.02547 \\
.02513\end{array}$ & $\begin{array}{l}24.75 \\
23.87 \\
23.13 \\
22.50 \\
21.97 \\
21.52 \\
21.15 \\
20.84 \\
20.58 \\
20.37\end{array}$ & $\begin{array}{l}.00216 \\
.00225 \\
.00235 \\
.00245 \\
.00256 \\
.00268 \\
.00280 \\
.00293 \\
.00306 \\
.00319\end{array}$ \\
\hline $\begin{array}{l}500 . \\
510 . \\
520 . \\
330 . \\
540 .\end{array}$ & $\begin{array}{l}21.9886 \\
21.3260 \\
20.7088 \\
20.1322 \\
19.5924\end{array}$ & $\begin{array}{l}.02417 \\
.02398 \\
.02372 \\
.02370 \\
.02380\end{array}$ & $\begin{array}{l}19.62 \\
19.51 \\
19.43 \\
19.37 \\
19.33\end{array}$ & $\begin{array}{l}.00342 \\
.00357 \\
.00371 \\
.00386 \\
.00401\end{array}$ & $\begin{array}{l}500 . \\
510 . \\
520 . \\
530 . \\
540 .\end{array}$ & $\begin{array}{l}23.1399 \\
22.4489 \\
21.0040 \\
21.2008 \\
20.6353\end{array}$ & $\begin{array}{l}.02489 \\
.02467 \\
.02448 \\
.02432 \\
.02420\end{array}$ & $\begin{array}{l}20.20 \\
20.06 \\
19.95 \\
19.06 \\
19.80\end{array}$ & $\begin{array}{l}.00332 \\
.00346 \\
.00359 \\
.00373 \\
.00387\end{array}$ \\
\hline
\end{tabular}

* Two Phase Boundary 
Table 7. Tronsport Propertles of Oxygen, Isobars, Engr. Units.

3800. Dsle Isobar

4000. DS1E Is ODE

Temp. Density Thermal Viscosity Thermal
Cond.

Temp. Density Thermat viscosity Thermal

\begin{tabular}{|c|c|c|c|c|c|c|c|c|c|}
\hline $\begin{array}{l}103.159 \\
105 . \\
110 . \\
115 . \\
120 . \\
125 . \\
130 . \\
135 . \\
140 . \\
145 .\end{array}$ & $\begin{array}{l}82.5513 \\
82.2992 \\
81.5841 \\
80.8859 \\
80.1920 \\
79.5005 \\
78.8100 \\
78.1196 \\
77.4285 \\
76.7362\end{array}$ & $\begin{array}{l}.12135 \\
.12036 \\
.11782 \\
.11546 \\
.11324 \\
.11113 \\
.10910 \\
.10714 \\
.10522 \\
.10334\end{array}$ & $\begin{array}{l}377.61 \\
367.77 \\
341.97 \\
317.71 \\
295.10 \\
274.17 \\
254.86 \\
237.18 \\
220.97 \\
206.14\end{array}$ & $\begin{array}{l}.00373 \\
.00371 \\
.00368 \\
.00364 \\
.00362 \\
.00359 \\
.00356 \\
.00354 \\
.00351 \\
.00349\end{array}$ & $\begin{array}{l}\text { * } 103.433 \\
105 . \\
110 . \\
115 . \\
120 . \\
125 . \\
130 . \\
135 . \\
140 . \\
145 .\end{array}$ & $\begin{array}{l}82.6013 \\
82.3788 \\
81.6760 \\
80.9807 \\
80.2902 \\
79.6024 \\
78.9159 \\
78.2299 \\
77.5435 \\
76.8561\end{array}$ & $\begin{array}{l}.12154 \\
.12070 \\
.11816 \\
.11581 \\
.11360 \\
.11150 \\
.10949 \\
.10754 \\
.10564 \\
.10377\end{array}$ & $\begin{array}{l}380.12 \\
371.63 \\
345.47 \\
320.91 \\
298.04 \\
276.88 \\
257.40 \\
239.53 \\
223.16 \\
208.19\end{array}$ & $\begin{array}{l}.00373 \\
.00372 \\
.00368 \\
.00365 \\
.00363 \\
.00360 \\
.00358 \\
.00355 \\
.00353 \\
.00350\end{array}$ \\
\hline $\begin{array}{l}150 . \\
155 . \\
160 . \\
165 . \\
170 . \\
175 . \\
180 . \\
185 . \\
190 . \\
195 .\end{array}$ & $\begin{array}{l}76.0422 \\
75.3461 \\
74.6477 \\
73.9467 \\
73.2427 \\
72.5355 \\
71.8248 \\
71.1104 \\
70.3919 \\
69.6690\end{array}$ & $\begin{array}{l}.10149 \\
.09966 \\
.09793 \\
.09602 \\
.09420 \\
.09239 \\
.09058 \\
.08877 \\
.08696 \\
.08515\end{array}$ & $\begin{array}{l}192.58 \\
180.19 \\
168.86 \\
158.50 \\
149.00 \\
140.30 \\
132.31 \\
124.96 \\
118.20 \\
111.96\end{array}$ & $\begin{array}{l}.00346 \\
.00344 \\
.00341 \\
.00338 \\
.00335 \\
.00332 \\
.00329 \\
.00325 \\
.00322 \\
.00318\end{array}$ & $\begin{array}{l}150 . \\
155 . \\
160 . \\
165 . \\
170 . \\
175 . \\
180 . \\
185 . \\
190 . \\
195 .\end{array}$ & $\begin{array}{l}76.1673 \\
75.4767 \\
74.7841 \\
74.0891 \\
73.3915 \\
72.6910 \\
71.9874 \\
71.2804 \\
70.5697 \\
69.8550\end{array}$ & $\begin{array}{l}.10193 \\
.10011 \\
.09831 \\
.09651 \\
.09471 \\
.09291 \\
.09112 \\
.08933 \\
.08753 \\
.08574\end{array}$ & $\begin{array}{l}194.51 \\
182.01 \\
170.59 \\
160.14 \\
150.57 \\
141.79 \\
133.74 \\
126.34 \\
119.52 \\
113.23\end{array}$ & $\begin{array}{l}.00348 \\
.00345 \\
.00343 \\
.00340 \\
.00337 \\
.00334 \\
.00331 \\
.00327 \\
.00324 \\
.00320\end{array}$ \\
\hline $\begin{array}{l}200 . \\
205 . \\
220 . \\
215 \\
220 . \\
225 . \\
230 . \\
2350 \\
240 . \\
245 .\end{array}$ & $\begin{array}{l}68.9413 \\
68.2084 \\
67.4700 \\
66.7258 \\
65.9752 \\
65.2178 \\
64.4533 \\
63.6812 \\
62.9011 \\
62.1127\end{array}$ & $\begin{array}{l}.08334 \\
.08133 \\
.07972 \\
.07792 \\
.07612 \\
.07433 \\
.07255 \\
.07078 \\
.06902 \\
.06728\end{array}$ & $\begin{array}{r}106.19 \\
100.85 \\
95.91 \\
91.31 \\
87.03 \\
63.04 \\
79.32 \\
75.84 \\
72.58 \\
69.51\end{array}$ & $\begin{array}{l}.00314 \\
.00310 \\
.00306 \\
.00302 \\
.00298 \\
.00293 \\
.00289 \\
.00284 \\
.00279 \\
.00275\end{array}$ & $\begin{array}{l}200 . \\
205 \\
210 \\
215 \\
220 \\
225 \\
230 \\
235 \\
240 \\
245 .\end{array}$ & $\begin{array}{l}69.1360 \\
68.4125 \\
67.6839 \\
66.9501 \\
66.2105 \\
65.4650 \\
64.7130 \\
63.9543 \\
63.1885 \\
62.4153\end{array}$ & $\begin{array}{l}.08394 \\
.08215 \\
.08036 \\
.07858 \\
.07680 \\
.07503 \\
.07326 \\
.07151 \\
.06978 \\
.06806\end{array}$ & $\begin{array}{r}107.43 \\
102.05 \\
97.07 \\
92.44 \\
88.14 \\
84.13 \\
80.39 \\
76.89 \\
73.61 \\
70.54\end{array}$ & $\begin{array}{l}.00317 \\
.00313 \\
.00309 \\
.00305 \\
.00301 \\
.00296 \\
.00292 \\
.00288 \\
.00283 \\
.00278\end{array}$ \\
\hline $\begin{array}{l}250 . \\
255 . \\
260 . \\
265 . \\
270 \\
275 . \\
280 . \\
285 . \\
290 \\
295 .\end{array}$ & $\begin{array}{l}61.3156 \\
60.5095 \\
59.6940 \\
58.8691 \\
58.0345 \\
57.1902 \\
56.3362 \\
55.4726 \\
54.5997 \\
53.7178\end{array}$ & $\begin{array}{l}.06556 \\
.06386 \\
.06218 \\
.06054 \\
.05893 \\
.05738 \\
.05586 \\
.05430 \\
.05278 \\
.05131\end{array}$ & $\begin{array}{l}66.64 \\
63.93 \\
61.38 \\
58.97 \\
56.69 \\
54.54 \\
52.50 \\
50.57 \\
48.73 \\
46.99\end{array}$ & $\begin{array}{l}.00270 \\
.00265 \\
.00261 \\
.00256 \\
.00252 \\
.00247 \\
.00243 \\
.00239 \\
.00234 \\
.00230\end{array}$ & $\begin{array}{l}250 . \\
255 . \\
260 . \\
265 . \\
270 . \\
2750^{\circ} \\
280 . \\
285 . \\
290 . \\
295 .\end{array}$ & $\begin{array}{l}61.6343 \\
60.8454 \\
60.0483 \\
59.2429 \\
58.4291 \\
57.6068 \\
56.7760 \\
55.9371 \\
55.0901 \\
54.2354\end{array}$ & $\begin{array}{l}.06636 \\
.06468 \\
.06302 \\
.06140 \\
.05981 \\
.05827 \\
.05676 \\
.05523 \\
.05373 \\
.05228\end{array}$ & $\begin{array}{l}67.65 \\
64.93 \\
62.38 \\
59.96 \\
57.68 \\
55.53 \\
53.49 \\
51.56 \\
49.72 \\
47.99\end{array}$ & $\begin{array}{l}.00274 \\
.00269 \\
.00265 \\
.00260 \\
.00256 \\
.00252 \\
.00248 \\
.00244 \\
.00239 \\
.00236\end{array}$ \\
\hline $\begin{array}{l}300 . \\
310 . \\
320 . \\
330 . \\
340 . \\
350 . \\
360 . \\
370 . \\
380 . \\
390 .\end{array}$ & $\begin{array}{l}52.8274 \\
51.0234 \\
49.1944 \\
47.3491 \\
43.4988 \\
43.6575 \\
41.8408 \\
40.0656 \\
38.3484 \\
36.7036\end{array}$ & $\begin{array}{l}.04988 \\
.04715 \\
.04462 \\
.04228 \\
.04015 \\
.03822 \\
.03650 \\
.03497 \\
.03362 \\
.03242\end{array}$ & $\begin{array}{l}45.34 \\
42.28 \\
39.52 \\
37.04 \\
34.79 \\
32.79 \\
30.99 \\
29.40 \\
28.00 \\
26.78\end{array}$ & $\begin{array}{l}.00226 \\
.00219 \\
.00213 \\
.00209 \\
.00205 \\
.00203 \\
.00203 \\
.00204 \\
.00207 \\
.00212\end{array}$ & $\begin{array}{l}300 . \\
310 . \\
320 . \\
330 . \\
340 . \\
350 . \\
360 . \\
370 . \\
380 . \\
390 .\end{array}$ & $\begin{array}{l}53.3736 \\
51.6305 \\
49.8669 \\
48.0905 \\
46.3112 \\
44.5404 \\
42.7913 \\
41.0780 \\
39.4143 \\
37.8128\end{array}$ & $\begin{array}{l}.05087 \\
.04817 \\
.04566 \\
.04333 \\
.04221 \\
.03928 \\
.03755 \\
.03600 \\
.03462 \\
.03340\end{array}$ & $\begin{array}{l}46.34 \\
43.29 \\
40.53 \\
38.05 \\
35.82 \\
33.81 \\
32.01 \\
30.41 \\
28.99 \\
27.74\end{array}$ & $\begin{array}{l}.00232 \\
.00225 \\
.00219 \\
.00214 \\
.00211 \\
.00209 \\
.00208 \\
.00209 \\
.00211 \\
.00215\end{array}$ \\
\hline $\begin{array}{l}400 . \\
410 . \\
420 . \\
430 . \\
440 . \\
450 . \\
460 . \\
470 . \\
480 . \\
490 .\end{array}$ & $\begin{array}{l}35.1423 \\
33.6716 \\
32.2946 \\
31.0107 \\
29.8173 \\
28.7096 \\
27.6820 \\
26.7284 \\
23.8428 \\
25.0191\end{array}$ & $\begin{array}{l}.03135 \\
.03041 \\
.02958 \\
.02883 \\
.02818 \\
.02760 \\
.02709 \\
.02664 \\
.02624 \\
.02590\end{array}$ & $\begin{array}{l}25.71 \\
24.80 \\
24.01 \\
23.33 \\
22.76 \\
22.27 \\
21.86 \\
21.51 \\
21.22 \\
20.98\end{array}$ & $\begin{array}{l}.00218 \\
.00226 \\
.00234 \\
.00244 \\
.00254 \\
.00265 \\
.00276 \\
.00287 \\
.00299 \\
.00312\end{array}$ & $\begin{array}{l}400 . \\
410 . \\
420 . \\
430 . \\
440 . \\
450 . \\
460 . \\
470 . \\
480 . \\
490 .\end{array}$ & $\begin{array}{l}36.2838 \\
34.8345 \\
33.4688 \\
32.1879 \\
30.9902 \\
29.8728 \\
28.8313 \\
27.8608 \\
26.9562 \\
26.1121\end{array}$ & $\begin{array}{l}.03232 \\
.03135 \\
.03049 \\
.02973 \\
.02905 \\
.02844 \\
.02791 \\
.02743 \\
.02701 \\
.02664\end{array}$ & $\begin{array}{l}26.64 \\
25.69 \\
24.86 \\
24.15 \\
23.54 \\
23.01 \\
22.57 \\
22.18 \\
21.86 \\
21.58\end{array}$ & $\begin{array}{l}.00220 \\
.00227 \\
.00234 \\
.00243 \\
.00252 \\
.00262 \\
.00272 \\
.00283 \\
.00294 \\
.00306\end{array}$ \\
\hline $\begin{array}{l}500 . \\
510 . \\
520 . \\
530 . \\
540 .\end{array}$ & $\begin{array}{l}24.2519 \\
23.5359 \\
22.8664 \\
22.2393 \\
21.6506\end{array}$ & $\begin{array}{l}.02561 \\
.02535 \\
.02314 \\
.02495 \\
.02480\end{array}$ & $\begin{array}{l}20.77 \\
20.61 \\
20.47 \\
20.36 \\
20.28\end{array}$ & $\begin{array}{l}.00324 \\
.00337 \\
.00349 \\
.00362 \\
.00375\end{array}$ & $\begin{array}{l}500 . \\
510 . \\
520 . \\
530 . \\
540 .\end{array}$ & $\begin{array}{l}25.3238 \\
24.5863 \\
23.8954 \\
23.2470 \\
22.6374\end{array}$ & $\begin{array}{l}.02632 \\
.02604 \\
.02579 \\
.02558 \\
.02540\end{array}$ & $\begin{array}{l}21.35 \\
21.16 \\
21.00 \\
20.87 \\
20.76\end{array}$ & $\begin{array}{l}.00317 \\
.00329 \\
.00341 \\
.00353 \\
.00365\end{array}$ \\
\hline
\end{tabular}

* Tro phose Boundarr 
Table 7. Transport Prodertles of Oxygen. Isobars, Engr. Units.

4500. Dsla Isobar

5000. Dsla Isobar

\begin{tabular}{|c|c|c|c|c|c|c|c|c|c|}
\hline $\begin{array}{c}\text { Teno. } \\
e\end{array}$ & $\begin{array}{l}\text { Density } \\
1 \mathrm{~b} / f+t * 3\end{array}$ & $\begin{array}{l}\text { Thermal } \\
\text { Cond. } \\
\text { erulft.h.R }\end{array}$ & $\begin{array}{c}\text { Viscosity } \\
\text { mIcro- } \\
\text { Iblftes }\end{array}$ & 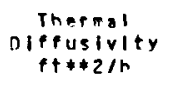 & Temp. & $\begin{array}{l}\text { Density } \\
\text { Ib/et+*3 }\end{array}$ & $\begin{array}{l}\text { Thermal } \\
\text { Cond. } \\
\text { BTulften.R }\end{array}$ & $\begin{array}{c}\text { viscosity } \\
\text { micro- } \\
\text { lb/ft.s }\end{array}$ & $\begin{array}{c}\text { Thermal } \\
\text { Dipfusivit } \\
\text { ft*2 } 2 / \mathrm{h}\end{array}$ \\
\hline $\begin{array}{l}106.115 \\
105 . \\
110 . \\
115 . \\
120 . \\
125 . \\
130 . \\
135 . \\
140 . \\
149 .\end{array}$ & $\begin{array}{l}82.7251 \\
82.6003 \\
81.9029 \\
81.2144 \\
80.5318 \\
79.9530 \\
79.1763 \\
78.5207 \\
77.8255 \\
77.1499\end{array}$ & $\begin{array}{l}.12202 \\
.12155 \\
.11933 \\
.11139 \\
.11451 \\
.11243 \\
.11045 \\
.10853 \\
.10354 \\
.10483\end{array}$ & $\begin{array}{l}186.30 \\
381.34 \\
354.27 \\
329.94 \\
305.41 \\
253.38 \\
263.70 \\
245.39 \\
228.63 \\
213.3 ?\end{array}$ & $\begin{array}{l}.00374 \\
.00373 \\
.00370 \\
.00367 \\
.00365 \\
.00363 \\
.00360 \\
.00358 \\
.00356 \\
.00354\end{array}$ & $\begin{array}{l}104.793 \\
105 . \\
110 . \\
115 . \\
120 . \\
125 . \\
130 . \\
135 . \\
140 . \\
145 .\end{array}$ & $\begin{array}{l}92.8470 \\
82.8181 \\
32.1256 \\
81.4435 \\
90.7684 \\
80.0979 \\
79.4305 \\
78.7648 \\
79.1001 \\
77.4357\end{array}$ & $\begin{array}{l}.12249 \\
.12239 \\
.11988 \\
.11756 \\
.11540 \\
.11335 \\
.11139 \\
.10950 \\
.10766 \\
.10587\end{array}$ & $\begin{array}{l}392.34 \\
391.14 \\
333.14 \\
337.02 \\
312.91 \\
270.50 \\
270.02 \\
251.25 \\
734.10 \\
318.64\end{array}$ & $\begin{array}{l}.00375 \\
.00375 \\
.00372 \\
.00359 \\
.00357 \\
.00365 \\
.00363 \\
.00361 \\
.00359 \\
.00357\end{array}$ \\
\hline $\begin{array}{l}150 . \\
155 . \\
160 . \\
165 . \\
170 \\
175 . \\
180 . \\
185 . \\
190 . \\
195 .\end{array}$ & $\begin{array}{l}76.4735 \\
75.7061 \\
75.117 ? \\
74.4365 \\
73.7541 \\
73.0605 \\
72.3925 \\
71.6929 \\
71.0006 \\
70.3052\end{array}$ & $\begin{array}{l}.10303 \\
.10124 \\
.09947 \\
.09771 \\
.04595 \\
.09419 \\
.09244 \\
.09048 \\
.08993 \\
.08717\end{array}$ & $\begin{array}{l}190.34 \\
186.56 \\
174.99 \\
164.72 \\
154.45 \\
145.50 \\
137.28 \\
129.73 \\
122.79 \\
115.39\end{array}$ & $\begin{array}{l}.00351 \\
.00340 \\
.00346 \\
.00344 \\
.00341 \\
.00338 \\
.00335 \\
.00332 \\
.00320 \\
.00324\end{array}$ & $\begin{array}{l}150 . \\
155 . \\
160 . \\
165 . \\
170 . \\
175 . \\
180 . \\
185 . \\
190 . \\
195 .\end{array}$ & $\begin{array}{l}75.7711 \\
75.1059 \\
75.4399 \\
74.7728 \\
74.1043 \\
73.4344 \\
72.7627 \\
72.0892 \\
71.4137 \\
70.7359\end{array}$ & $\begin{array}{l}.10410 \\
.10235 \\
.10061 \\
.09888 \\
.09716 \\
.09544 \\
.09372 \\
.09200 \\
.00028 \\
.09857\end{array}$ & $\begin{array}{l}204.14 \\
181.09 \\
179.17 \\
169.27 \\
158.30 \\
149.16 \\
140.79 \\
133.09 \\
126.01 \\
117.48\end{array}$ & $\begin{array}{l}.00355 \\
.00352 \\
.00350 \\
.00348 \\
.00345 \\
.00343 \\
.00340 \\
.00337 \\
.00334 \\
.00331\end{array}$ \\
\hline $\begin{array}{l}200 . \\
2050 \\
210 \\
2150 \\
220 \\
2250 \\
230 \\
2350 \\
240 . \\
2450\end{array}$ & $\begin{array}{l}50.6066 \\
18.9065 \\
68.1985 \\
67.4887 \\
68.7745 \\
66.0558 \\
35.3323 \\
64.6037 \\
63.8398 \\
33.1304\end{array}$ & $\begin{array}{l}.08542 \\
.08367 \\
.08192 \\
.08018 \\
.07844 \\
.07672 \\
.07500 \\
.07330 \\
.07150 \\
.06993\end{array}$ & $\begin{array}{r}110.47 \\
105.00 \\
99.93 \\
95.73 \\
90.86 \\
86.79 \\
82.99 \\
79.45 \\
76.13 \\
73.02\end{array}$ & $\begin{array}{l}.00322 \\
.00319 \\
.00315 \\
.00311 \\
.00308 \\
.00304 \\
.00300 \\
.00295 \\
.00291 \\
.00287\end{array}$ & $\begin{array}{l}200 . \\
205 . \\
210 . \\
215 . \\
220 . \\
225 . \\
230 . \\
235 . \\
240 . \\
245 .\end{array}$ & $\begin{array}{l}70.0558 \\
69.3730 \\
68.6875 \\
67.9989 \\
67.3073 \\
65.6122 \\
35.9136 \\
65.2112 \\
64.5050 \\
63.7947\end{array}$ & $\begin{array}{l}.08555 \\
.08514 \\
.08343 \\
.08173 \\
.08003 \\
.07834 \\
.07666 \\
.07500 \\
.07335 \\
.07171\end{array}$ & $\begin{array}{r}-113.46 \\
107.89 \\
102.74 \\
97.95 \\
93.51 \\
89.38 \\
85.52 \\
91.73 \\
78.56 \\
75.61\end{array}$ & $\begin{array}{l}.00328 \\
.00325 \\
.00321 \\
.00318 \\
.00314 \\
.00310 \\
.00307 \\
.00303 \\
.00299 \\
.00295\end{array}$ \\
\hline $\begin{array}{l}250 . \\
255 . \\
260 . \\
265 . \\
270 . \\
275 . \\
280 . \\
285 . \\
290 . \\
295 .\end{array}$ & $\begin{array}{l}62.3954 \\
61.6345 \\
60.8776 \\
60.1148 \\
59.3458 \\
58.5709 \\
57.7901 \\
57.0035 \\
56.2115 \\
55.4142\end{array}$ & $\begin{array}{l}.06828 \\
.06464 \\
.06503 \\
.06345 \\
.06191 \\
.06040 \\
.05893 \\
.05744 \\
.05599 \\
.05458\end{array}$ & $\begin{array}{l}70.10 \\
67.36 \\
54.78 \\
6 ? .35 \\
60.06 \\
57.89 \\
55.84 \\
53.01 \\
52.07 \\
50.33\end{array}$ & $\begin{array}{l}.00283 \\
.00279 \\
.00275 \\
.00270 \\
.00256 \\
.00263 \\
.00259 \\
.00255 \\
.00251 \\
.00247\end{array}$ & $\begin{array}{l}250 . \\
255 . \\
260 . \\
255 . \\
270 . \\
275 . \\
280 . \\
285 . \\
290 . \\
295 .\end{array}$ & $\begin{array}{l}63.0803 \\
62.3617 \\
61.6387 \\
60.9114 \\
60.1798 \\
59.4440 \\
58.7040 \\
57.9601 \\
57.2124 \\
56.4613\end{array}$ & $\begin{array}{l}.07010 \\
.06850 \\
.06693 \\
.06539 \\
.06388 \\
.06241 \\
.05096 \\
.05951 \\
.05810 \\
.05672\end{array}$ & $\begin{array}{l}72.63 \\
69.68 \\
67.08 \\
64.62 \\
62.31 \\
50.12 \\
58.06 \\
56.11 \\
54.27 \\
52.52\end{array}$ & $\begin{array}{l}.00291 \\
.00287 \\
.00283 \\
.00280 \\
.00276 \\
.00272 \\
.00269 \\
.00255 \\
.00252 \\
.00258\end{array}$ \\
\hline $\begin{array}{l}300 . \\
310 . \\
320 . \\
330 . \\
340 . \\
350 . \\
360 . \\
370 . \\
380 . \\
390 .\end{array}$ & $\begin{array}{l}54.6122 \\
52.9958 \\
51.3670 \\
49.7318 \\
48.0973 \\
46.4715 \\
44.8334 \\
43.2822 \\
41.7375 \\
40.2381\end{array}$ & $\begin{array}{l}.05370 \\
.05058 \\
.04811 \\
.04582 \\
.04371 \\
.04178 \\
.04003 \\
.03844 \\
.03702 \\
.03575\end{array}$ & $\begin{array}{l}48.68 \\
45.04 \\
42.89 \\
40.41 \\
38.17 \\
34.16 \\
34.35 \\
32.72 \\
31.26 \\
29.97\end{array}$ & $\begin{array}{l}.00244 \\
.00238 \\
.00232 \\
.00227 \\
.00224 \\
.00221 \\
.00220 \\
.00220 \\
.00221 \\
.00224\end{array}$ & $\begin{array}{l}300 . \\
310 . \\
320 . \\
330 . \\
340 . \\
350 . \\
360 . \\
370 . \\
390 . \\
390 .\end{array}$ & $\begin{array}{l}55.7070 \\
54.1909 \\
52.6677 \\
51.1423 \\
49.6200 \\
48.1069 \\
46.6095 \\
45.1345 \\
43.6886 \\
42.2784\end{array}$ & $\begin{array}{l}.05538 \\
.05281 \\
.05039 \\
.04813 \\
.04604 \\
.04411 \\
.04234 \\
.04073 \\
.03928 \\
.03796\end{array}$ & $\begin{array}{l}50.86 \\
47.80 \\
45.04 \\
42.56 \\
40.31 \\
35.28 \\
36.46 \\
34.81 \\
33.32 \\
31.99\end{array}$ & $\begin{array}{l}.00255 \\
.00249 \\
.00244 \\
.00239 \\
.00236 \\
.00233 \\
.00231 \\
.00231 \\
.00231 \\
.00232\end{array}$ \\
\hline $\begin{array}{l}400 . \\
410 . \\
420 . \\
430 . \\
440 . \\
450 . \\
460 . \\
470 . \\
480 . \\
490 .\end{array}$ & $\begin{array}{l}38.7719 \\
37.4053 \\
36.0827 \\
34.8269 \\
33.6386 \\
32.5173 \\
31.4513 \\
30.4678 \\
29.5336 \\
28.6553\end{array}$ & $\begin{array}{l}.03461 \\
.03359 \\
.03248 \\
.03186 \\
.03113 \\
.03047 \\
.02988 \\
.02935 \\
.02897 \\
.02844\end{array}$ & $\begin{array}{l}29.81 \\
27.79 \\
26.90 \\
26.11 \\
25.41 \\
24.82 \\
24.28 \\
23.82 \\
23.43 \\
23.08\end{array}$ & $\begin{array}{l}.00227 \\
.00232 \\
.00238 \\
.00244 \\
.00252 \\
.00259 \\
.00268 \\
.00277 \\
.00286 \\
.00293\end{array}$ & $\begin{array}{l}400 . \\
410 . \\
420 . \\
430 . \\
440 . \\
450 . \\
460 . \\
470 . \\
480 . \\
490 .\end{array}$ & $\begin{array}{l}40.9098 \\
39.5877 \\
38.3164 \\
37.0987 \\
35.9362 \\
34.8296 \\
33.7784 \\
32.7816 \\
31.8372 \\
30.9430\end{array}$ & $\begin{array}{l}.03678 \\
.03571 \\
.03475 \\
.03388 \\
.03310 \\
.03240 \\
.03176 \\
.03118 \\
.03065 \\
.03018\end{array}$ & $\begin{array}{l}30.80 \\
29.73 \\
28.77 \\
27.93 \\
27.17 \\
26.51 \\
25.92 \\
25.40 \\
24.94 \\
24.54\end{array}$ & $\begin{array}{l}.00235 \\
.00238 \\
.00243 \\
.00248 \\
.00254 \\
.00260 \\
.00267 \\
.00274 \\
.00282 \\
.00290\end{array}$ \\
\hline $\begin{array}{l}500 . \\
520 . \\
520 . \\
530 . \\
540 .\end{array}$ & $\begin{array}{l}27.8293 \\
27.0518 \\
26.3195 \\
25.6290 \\
24.9771\end{array}$ & $\begin{array}{l}.02806 \\
.02772 \\
.02741 \\
.02714 \\
.02690\end{array}$ & $\begin{array}{l}22.78 \\
22.53 \\
22.31 \\
22.12 \\
21.97\end{array}$ & $\begin{array}{l}.00306 \\
.00316 \\
.00326 \\
.00336 \\
.00346\end{array}$ & $\begin{array}{l}500 . \\
510 . \\
520 . \\
530 . \\
540 .\end{array}$ & $\begin{array}{l}30.0967 \\
29.2956 \\
28.5369 \\
27.8182 \\
27.1367\end{array}$ & $\begin{array}{l}.02974 \\
.02935 \\
.02899 \\
.02867 \\
.02838\end{array}$ & $\begin{array}{l}24.18 \\
23.87 \\
23.60 \\
23.37 \\
23.17\end{array}$ & $\begin{array}{l}.00299 \\
.00307 \\
.00316 \\
.00325 \\
.00334\end{array}$ \\
\hline
\end{tabular}

* Two phase goundary 
Table 7. Transoort Properzles of Dxygen, Isobars, Engr. Units.

b000. Fsla Isobar

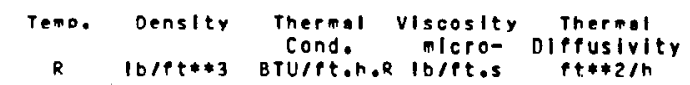

$+106.14083 .0854$

110.

$115.02 .35 \mathrm{~A}$

120. 61.2270

$12 \%$. 80.5720

$\begin{array}{ll}130 . & 79.9215 \\ 135 & 79.2741\end{array}$

$\begin{array}{ll}1350 & 79.7741 \\ 140 . & 78.5289 \\ 1450 & 77.9850\end{array}$

150. $\quad 77.3419$

$\begin{array}{ll}\text { 150. } & 77.3419 \\ 155 . & 73.5993\end{array}$

$\begin{array}{ll}155 . & 75.5993 \\ 16 \mathrm{C} . & 76.0547 \\ 165 . & 75.4141\end{array}$

165.
170. 74.47111

$\begin{array}{ll}170 . & 74.7711 \\ 175 . & 74.1276\end{array}$

$180 . \quad 73.4835$

100.72 .1029

$200 . \quad 70.8944$

$\begin{array}{ll}20 n . & 70.8984 \\ 205 . & 70.7494 \\ 210 . & 79.3090\end{array}$

$\begin{array}{ll}210 . & 09.3990 \\ 215 . & 0.9472\end{array}$

$\begin{array}{ll}215 . & 68.9472 \\ 220 . & 68.2939\end{array}$

275.67 .6389

230. 66.9821

$\begin{array}{ll}235 . & 66.3235 \\ 240 . & 65.6631\end{array}$

?.45. 65.0005

.12345

.12155

.11926

.11714

.11514

.11140

.10942
.10788

.10618

.10449

.10282

.10116

.09795

.09620

.09454

.09289

.08950

. 08795

.08631

08467

.08304
.08142

.07981

$.079 ? 1$

.07663
.07506

lofetes

7000. osla Isobar

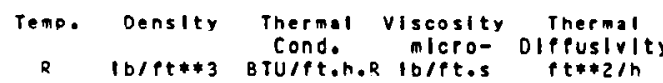

R Ib/ft**3 Biulftin, ib/ftes olf fusivity

$t+2 / \mathrm{h}$

$\begin{array}{lll}* 107.474 & 83.3167 \\ 110 . & 82.9774 \\ 115 . & 82.3168 & \\ 120 . & 81.6676 & \\ 125 . & 81.0265 & \\ 130 . & 80.3913 & \\ 135 . & 79.7604 & \\ 140 . & 79.1326 & \\ 145 . & 78.5072\end{array}$

12430

12439
.12318

.12092

.11883

.11687

11323

.11151

7 B. 5072

150. $77.8834 \quad .10818$

155. $77.2610 \quad .10655$

160. $76.6394 \quad .10494$

$\begin{array}{lll}165 . & 75.0195 & .10334 \\ 170 . & 75.3980 & .10175\end{array}$

$\begin{array}{lll}175 . & 74.7778 & .10015\end{array}$

$180 . \quad 74.1578 \quad .09856$

$185 . \quad 73.5379 \quad .09697$

$\begin{array}{lll}170 . & 72.9179 & .09538 \\ 175 . & 72.2970 & .09370\end{array}$

200. $\quad 71.6777 \quad .09220$

205. 71.0572

210.70 .4364

21500.6364

220.069 .1937

225. 69.5716

230.69 .9490

235. 67.3258

240. 65.7020

245. 35.0775

.09220
.09061

.08903

.08745

.08588

.08431

.09275

.08121

.07968
.07816

$250 . \quad 55.4523 \quad .07667$

$260.04 .2001 \quad 07519$

$265.03 .5730 \quad .07373$

$265 . \quad 63.5730 \quad .07229$

$270 . \quad 62.9453 \quad .07088$

275. 62.3171 .06950

$\begin{array}{lll}280 . & 61.0986 & .03814 \\ 285 . & 31.0597 & .05680\end{array}$

$\begin{array}{lll}295 . & 31.0597 & .05680 \\ 290 . & 60.4306 & .06549\end{array}$

$\begin{array}{lll}205 . & 59.4015 & .06549\end{array}$

300. $50.1725 \quad .06295$

$310 . \quad 57.9159$

$320 . \quad 56.6625$

$330 . \quad 55.4144$

340.54 .1741

340.52 .1741

360.52 .9460

$360.51 .726 \mathrm{R}$

$\begin{array}{ll}370 . & 50.5251 \\ 390 . & 49.3416\end{array}$

$300 . \quad 49.1798$

.06053

.05824

.05607

.05404

.05214

04871

.04871

.04719

$415.12 \quad .00379$

$399.26 \quad .00378$

$369.78 \quad .00376$

$362.72 \quad .00375$

$317.97 \quad .00374$

$295.36 \quad .00372$

$274.73 \quad .00371$

$255.94 \quad .00370$

238.03 .00358

$223.24 \quad .00367$

$209.03 \quad .00355$

$195.07 \quad .00364$

$184.24 \quad .00362$

$173.44 \quad .00360$

$153.54 \quad .00358$

$154.48 \quad .00356$

$146.17 \quad .00354$

$133.52 \quad .00352$

$125.00 \quad .00347$

$119.01 \quad .00345$

$113.48 \quad .00342$

$138.35 \quad .00340$

$103.57 \quad .00337$

$89.15 \quad .00334$

$95.05 \quad .00331$

$91.21 \quad .00328$

$\begin{array}{ll}87.63 & .00325 \\ 84.29 & .00322\end{array}$

$81.15 \quad .00319$

$78.22 \quad .00315$

$75.46 \quad .00313$

$72.97 \quad .00310$

$70.44 \quad .00307$

$68.15 \quad .00304$

$65.98 \quad .00321$

$63.94 \quad .00278$

$\begin{array}{ll}62.01 & .00295 \\ 50.19 & .00293\end{array}$

58.45 .00290

$55.28 \quad .00285$

32.42 .00281

$49.93 \quad .00277$

$47.49 \quad .00273$

$45.38 \quad .00271$

$43.46 \quad .00258$

$41.71 \quad .00267$

$40.13 \quad .00256$

$\begin{array}{lllll}400 . & 47.0390 & .04447 & 37.37 & .00240 \\ 410 . & 45.9745 & .04327 & 36.18 & .00267\end{array}$

$420.044 .9370 \quad .04216 \quad 35.09 \quad .00268$

$430 . \quad 43.7783 \quad .04115 \quad 34.10 \quad .07270$

$440.4 \begin{array}{llll}42.7496 & .04021 & 33.20 & .00273\end{array}$

450. $41.7519 \quad .03935 \quad 32.38 \quad .00270$

$450.40 .7861 \quad 03856 \quad 31.040 .00270$

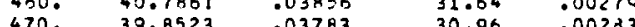

480. $39.9508 \quad .03783 \quad 30.95 \quad .002+3$

$\begin{array}{lllll}480 . & 38.9508 & .03715 & 30.34 & .0027\end{array}$

500. $\quad 37.2437 \quad 0.03594 \quad 29.28 \quad .00278$

$510 . \quad 36.4372 \quad .03540 \quad 28.81 \quad .00321$

520 . $35.6610 \quad .03450 \quad 28.40 \quad$. CC3O

$\begin{array}{lllll}330 . & 34.9143 & .03443 & 28.02 & .00311 \\ 540 . & 36.1952 & .03399 & 27.57 & .03317\end{array}$

* Two Phase Boundary 
Table 7. Transoopt Propertles of Dxygen, Isobars, Engr. Unlts.

8000 . osta Isobor

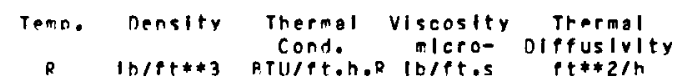

9000.0510 Isobar

Temp. Density Thermal Viscosity Thermal

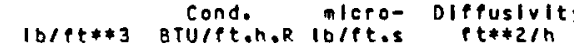

\begin{tabular}{|c|c|c|c|c|c|c|c|c|c|}
\hline $\begin{array}{l}109.790 \\
110 . \\
125 . \\
120 . \\
125 . \\
130 . \\
135 . \\
140 . \\
145 .\end{array}$ & $\begin{array}{l}83.5415 \\
93.3920 \\
87.7307 \\
07.0917 \\
81.4331 \\
90.4416 \\
90.7254 \\
79.6137 \\
79.0050\end{array}$ & $\begin{array}{l}.12534 \\
.12477 \\
.12254 \\
.12048 \\
.11856 \\
.11675 \\
.11501 \\
.11334 \\
.111171\end{array}$ & $\begin{array}{l}425.69 \\
417.72 \\
386.43 \\
357.87 \\
331.82 \\
308.09 \\
296.49 \\
256.85 \\
248.98\end{array}$ & $\begin{array}{l}.00380 \\
.00380 \\
.00370 \\
.00378 \\
.00378 \\
.00377 \\
.00376 \\
.00375 \\
.00374\end{array}$ & $\begin{array}{l}* 110.105 \\
115 . \\
120 . \\
125 . \\
130 . \\
135 . \\
140 . \\
145 .\end{array}$ & $\begin{array}{l}83.7501 \\
93.1295 \\
82.5007 \\
81.8833 \\
81.2743 \\
80.6718 \\
80.0743 \\
79.4808\end{array}$ & $\begin{array}{l}.12628 \\
.12412 \\
.12209 \\
.12021 \\
.11643 \\
.11674 \\
.11512 \\
.11354\end{array}$ & $\begin{array}{l}435.73 \\
403.28 \\
373.14 \\
345.75 \\
320.87 \\
298.27 \\
277.75 \\
259.11\end{array}$ & $\begin{array}{l}.00382 \\
.00382 \\
.00382 \\
.00381 \\
.00381 \\
.00380 \\
.00379 \\
.00379\end{array}$ \\
\hline $\begin{array}{l}150 . \\
155 . \\
160 . \\
165 . \\
170 . \\
175 . \\
180 . \\
185 . \\
190 . \\
195 .\end{array}$ & $\begin{array}{l}78.3987 \\
77.7945 \\
77.1919 \\
76.5903 \\
75.9900 \\
75.3905 \\
74.7920 \\
74.1940 \\
73.5067 \\
73.0000\end{array}$ & $\begin{array}{l}.11012 \\
.10855 \\
.10699 \\
.10545 \\
.10391 \\
.10237 \\
.10083 \\
.09630 \\
.09776 \\
.0962 ?\end{array}$ & $\begin{array}{l}232.72 \\
217.91 \\
204.42 \\
192.11 \\
180.87 \\
170.59 \\
161.17 \\
152.53 \\
144.40 \\
137.30\end{array}$ & $\begin{array}{l}.00373 \\
.00371 \\
.00370 \\
.00369 \\
.00367 \\
.00366 \\
.00364 \\
.00362 \\
.00360 \\
.00358\end{array}$ & $\begin{array}{l}150 . \\
155 . \\
160 . \\
165 . \\
170 . \\
175 . \\
180 . \\
185 . \\
190 . \\
195 .\end{array}$ & $\begin{array}{l}78.8905 \\
78.3027 \\
77.7171 \\
77.1333 \\
76.5512 \\
75.9704 \\
75.3910 \\
74.8127 \\
74.2355 \\
73.6594\end{array}$ & $\begin{array}{l}.11200 \\
.11047 \\
.10897 \\
.10748 \\
.10599 \\
.10450 \\
.10302 \\
.10153 \\
.10005 \\
.09856\end{array}$ & $\begin{array}{l}242.17 \\
226.75 \\
212.71 \\
199.91 \\
188.23 \\
177.55 \\
167.77 \\
158.81 \\
150.58 \\
143.01\end{array}$ & $\begin{array}{l}.00378 \\
.00377 \\
.00376 \\
.00375 \\
.00373 \\
.00372 \\
.00371 \\
.00369 \\
.00368 \\
.00366\end{array}$ \\
\hline $\begin{array}{l}200 . \\
205 . \\
210 . \\
215 . \\
220 . \\
225 . \\
230 . \\
235 . \\
240 . \\
245 .\end{array}$ & $\begin{array}{l}77.4037 \\
71.8080 \\
71.2126 \\
70.6176 \\
70.0230 \\
69.4286 \\
48.8340 \\
68.2409 \\
67.6474 \\
67.0541\end{array}$ & $\begin{array}{l}.09469 \\
.09315 \\
.09142 \\
.09009 \\
.08956 \\
.09705 \\
.08554 \\
.09404 \\
.08255 \\
.08108\end{array}$ & $\begin{array}{r}130.57 \\
124.36 \\
118.62 \\
113.31 \\
108.38 \\
103.80 \\
99.54 \\
95.57 \\
91.87 \\
88.42\end{array}$ & $\begin{array}{l}.00354 \\
.00354 \\
.00351 \\
.00349 \\
.00347 \\
.00344 \\
.00341 \\
.00339 \\
.00336 \\
.00333\end{array}$ & $\begin{array}{l}200 . \\
205 \\
210 \\
215 \\
220 \\
2250 \\
230 \\
2350 \\
240 . \\
245 .\end{array}$ & $\begin{array}{l}73.0843 \\
72.5102 \\
71.9371 \\
71.3648 \\
70.7935 \\
70.2231 \\
69.6535 \\
69.0849 \\
68.5170 \\
67.9501\end{array}$ & $\begin{array}{l}.09707 \\
.09558 \\
.09409 \\
.09261 \\
.09113 \\
.08965 \\
.08819 \\
.08673 \\
.08528 \\
.08385\end{array}$ & $\begin{array}{r}136.03 \\
129.60 \\
123.65 \\
118.14 \\
113.04 \\
108.30 \\
103.90 \\
99.80 \\
95.97 \\
92.40\end{array}$ & $\begin{array}{l}.00364 \\
.00362 \\
.00360 \\
.00358 \\
.00356 \\
.00353 \\
.00351 \\
.00349 \\
.00346 \\
.00344\end{array}$ \\
\hline $\begin{array}{l}250 . \\
255 . \\
260 . \\
265 . \\
270 . \\
275 . \\
280 . \\
285 . \\
290 . \\
295 .\end{array}$ & $\begin{array}{l}63.4511 \\
65.8682 \\
65.2759 \\
64.6837 \\
64.0019 \\
63.5005 \\
62.9095 \\
62.3192 \\
61.7295 \\
61.1405\end{array}$ & $\begin{array}{l}.07963 \\
.07819 \\
.07677 \\
.07537 \\
.07400 \\
.07265 \\
.07133 \\
.07002 \\
.06874 \\
.06748\end{array}$ & $\begin{array}{l}85.18 \\
82.15 \\
79.31 \\
76.64 \\
74.13 \\
71.77 \\
69.55 \\
67.45 \\
65.46 \\
33.59\end{array}$ & $\begin{array}{l}.00331 \\
.00328 \\
.00325 \\
.00323 \\
.00320 \\
.00317 \\
.00315 \\
.00312 \\
.00309 \\
.00307\end{array}$ & $\begin{array}{l}250 . \\
255 . \\
260 . \\
265 . \\
270 . \\
275 . \\
280 . \\
285 . \\
290 . \\
295 .\end{array}$ & $\begin{array}{l}67.3841 \\
66.8189 \\
66.2547 \\
65.6914 \\
65.1290 \\
64.5677 \\
64.0075 \\
63.4485 \\
62.8906 \\
62.3341\end{array}$ & $\begin{array}{l}.08243 \\
.08103 \\
.07964 \\
.07828 \\
.07693 \\
.07561 \\
.07431 \\
.07303 \\
.07178 \\
.07055\end{array}$ & $\begin{array}{l}89.06 \\
85.93 \\
83.00 \\
80.24 \\
77.65 \\
75.22 \\
72.93 \\
70.76 \\
68.72 \\
66.79\end{array}$ & $\begin{array}{l}.00341 \\
.00339 \\
.00336 \\
.00334 \\
.00331 \\
.00329 \\
.00327 \\
.00324 \\
.00322 \\
.00320\end{array}$ \\
\hline $\begin{array}{l}300 . \\
310 . \\
320 . \\
330 . \\
340 . \\
350 . \\
360 . \\
370 . \\
380 . \\
390 .\end{array}$ & $\begin{array}{l}60.5525 \\
59.3797 \\
58.2124 \\
57.0522 \\
55.9005 \\
54.7593 \\
53.5304 \\
52.5157 \\
51.4169 \\
50.3359\end{array}$ & $\begin{array}{l}.06626 \\
.06389 \\
.06164 \\
.05951 \\
.05750 \\
.05561 \\
.05384 \\
.05218 \\
.05064 \\
.04920\end{array}$ & $\begin{array}{l}61.82 \\
58.55 \\
55.61 \\
52.95 \\
50.55 \\
48.38 \\
46.40 \\
44.61 \\
42.97 \\
41.48\end{array}$ & $\begin{array}{l}.00305 \\
.00300 \\
.00296 \\
.00292 \\
.00289 \\
.00286 \\
.00284 \\
.00282 \\
.00281 \\
.00280\end{array}$ & $\begin{array}{l}300 . \\
310 . \\
320 . \\
330 . \\
340 . \\
350 . \\
360 . \\
370 . \\
380 . \\
390 .\end{array}$ & $\begin{array}{l}61.7789 \\
60.6732 \\
59.5745 \\
58.4838 \\
57.4024 \\
56.3315 \\
55.2725 \\
54.2266 \\
53.1954 \\
32.1799\end{array}$ & $\begin{array}{l}.06934 \\
.06702 \\
.06480 \\
.06270 \\
.06071 \\
.05883 \\
.05706 \\
.05540 \\
.05384 \\
.05238\end{array}$ & $\begin{array}{l}64.97 \\
61.60 \\
38.58 \\
55.85 \\
53.38 \\
51.14 \\
49.11 \\
47.26 \\
45.57 \\
44.03\end{array}$ & $\begin{array}{l}.00317 \\
.00313 \\
.00309 \\
.00306 \\
.00302 \\
.00300 \\
.00297 \\
.00295 \\
.00294 \\
.00293\end{array}$ \\
\hline $\begin{array}{l}400 . \\
410 . \\
420 . \\
430 . \\
440 . \\
450 . \\
460 . \\
470 . \\
480 . \\
490 .\end{array}$ & $\begin{array}{l}49.2744 \\
48.2338 \\
47.2155 \\
46.2207 \\
45.2505 \\
44.3055 \\
43.3844 \\
42.4937 \\
41.6274 \\
40.7877\end{array}$ & $\begin{array}{l}.04786 \\
.04661 \\
.04546 \\
.04439 \\
.04340 \\
.04248 \\
.04163 \\
.04084 \\
.04010 \\
.03941\end{array}$ & $\begin{array}{l}40.12 \\
38.88 \\
37.74 \\
36.70 \\
35.75 \\
34.88 \\
34.08 \\
33.35 \\
32.08 \\
32.07\end{array}$ & $\begin{array}{l}.00280 \\
.00280 \\
.00281 \\
.00282 \\
.00284 \\
.00286 \\
.00288 \\
.00291 \\
.00294 \\
.00297\end{array}$ & $\begin{array}{l}400 . \\
410 . \\
420 . \\
430 . \\
440 . \\
450 . \\
460 . \\
470 . \\
490 . \\
490 .\end{array}$ & $\begin{array}{l}51.1815 \\
50.2013 \\
49.2403 \\
48.2994 \\
47.3794 \\
46.4807 \\
45.6041 \\
44.7497 \\
43.9178 \\
43.1085\end{array}$ & $\begin{array}{l}.05101 \\
.04974 \\
.04855 \\
.04743 \\
.04640 \\
.04543 \\
.04452 \\
.04368 \\
.04288 \\
.04214\end{array}$ & $\begin{array}{l}42.62 \\
41.33 \\
40.15 \\
39.06 \\
38.07 \\
37.15 \\
36.31 \\
35.54 \\
34.83 \\
34.17\end{array}$ & $\begin{array}{l}.00292 \\
.00292 \\
.00293 \\
.00293 \\
.00294 \\
.00296 \\
.00297 \\
.00299 \\
.00301 \\
.00304\end{array}$ \\
\hline $\begin{array}{l}500 . \\
510 . \\
520 . \\
530 . \\
540 .\end{array}$ & $\begin{array}{l}39.9744 \\
39.1873 \\
38.4259 \\
37.6898 \\
36.9783\end{array}$ & $\begin{array}{l}.03877 \\
.03817 \\
.03760 \\
.03707 \\
.03658\end{array}$ & $\begin{array}{l}31.51 \\
31.00 \\
30.53 \\
30.10 \\
29.71\end{array}$ & $\begin{array}{l}.00301 \\
.00304 \\
.00308 \\
.00312 \\
.00317\end{array}$ & $\begin{array}{l}500 . \\
510 . \\
520 . \\
530 . \\
540 .\end{array}$ & $\begin{array}{l}42.3218 \\
41.5576 \\
40.8155 \\
40.0954 \\
39.3967\end{array}$ & $\begin{array}{l}.04144 \\
.04078 \\
.04016 \\
.03958 \\
.03903\end{array}$ & $\begin{array}{l}33.57 \\
33.02 \\
32.51 \\
32.04 \\
31.61\end{array}$ & $\begin{array}{l}.00307 \\
.00309 \\
.00313 \\
.00316 \\
.00319\end{array}$ \\
\hline
\end{tabular}


Table 7. Transoort Prodertles of Oxygen, Isobars, Engr. Units.

10000. psta Isobar

11000. psite Isobar

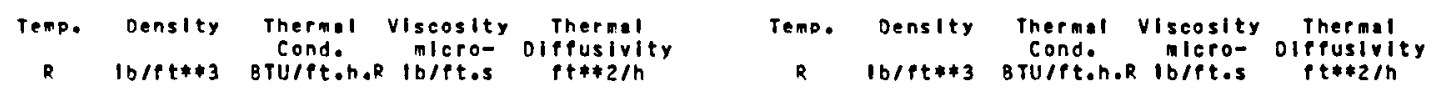

.08510
.08372

.08372
.08237

.08237

.08103

.07842

.07714

.07588

.07465

$92.80 \quad .00351$

$\begin{array}{ll}89.57 & .00349 \\ 86.54 & .00347\end{array}$

$\begin{array}{ll}86.54 & .00347 \\ 83.70 & .00344\end{array}$

$91.03 \quad .00342$

$78.52 \quad .00340$

$76.15 \quad .00337$

$73.93 \quad .00335$

$69.83 \quad .00331$

$295 . \quad 63.4140$

.07225

3c0. 62.8861

.06996

.06759

.06569
.06371

.06184

.06007

.05840

.05683

67.95

.00329

$64.49 \quad .00325$

$61.37 \quad .00321$

$58.57 \quad .0031$

$\begin{array}{ll}56.03 & .00315 \\ 53.72 & .00312\end{array}$

$\begin{array}{ll}53.72 & .00312 \\ 51.63 & .00310\end{array}$

$\begin{array}{ll}51.63 & .00310 \\ 49.72 & .00300\end{array}$

$47.98 \quad .00306$

$46.39 \quad .00305$

$390 . \quad 53.7931$

.05307

$44.94 \quad .00304$

$42.37 \quad .00304$

$41.25 \quad 0.00304$

$4.25 \quad .00304$

$40.21 \quad .00304$

$39.25 \quad .00305$

$\begin{array}{ll}38.37 & .00306 \\ 37.56 & .00308\end{array}$

$\begin{array}{ll}37.56 & .00308 \\ 36.81 & .00309\end{array}$

$36.12 \quad .00311$

$\begin{array}{lll}* 112.688 & 84.1803 & .12815 \\ 115 . & 83.8907 & .12718 \\ 120 . & 83.2779 & .12520 \\ 125 . & 82.6796 & .12338 \\ 130 . & 82.0923 & .12168 \\ 135 . & 81.5134 & .12007 \\ 140 . & 80.9412 & .11853 \\ 145 . & 80.3743 & .11704\end{array}$

$454.28 \quad .00385$

$\begin{array}{ll}437.57 & .00386 \\ 404.08 & .00387\end{array}$

$\begin{array}{ll}404.08 & .00387 \\ 373.87 & .00387\end{array}$

$346.58 \quad .00387$

$321.91 \quad .00388$

$\begin{array}{ll}299.57 & .00388 \\ 279.33 & .00397\end{array}$

145

150. 79.8118

.11704

$260.98 \quad .00387$

$\begin{array}{lllll}155 . & 79.2530 & .11416 & 244.31 & .00387\end{array}$

165. 78.1444 $\quad .11135 \quad 215.35 \quad .00386$

$\begin{array}{lllll}170 . & 77.5938 & .10996 & 202.77 & .00385\end{array}$

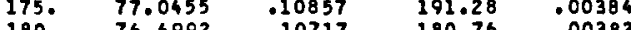

$\begin{array}{lllll}180 . & 76.4992 & .10717 & 180.76 & .00383\end{array}$

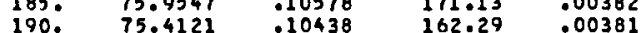

$\begin{array}{lllll}190 . & 75.4121 & .10438 & 162.29 & .00381 \\ 195 . & 74.8712 & .10298 & 154.17 & .00380\end{array}$

$\begin{array}{lllll}200 . & 74.3321 & .10158 & 146.69 & .00378 \\ 205 . & 73.7946 & .10017 & 139.79 & .00377 \\ 210 . & 73.2587 & .09877 & 133.42 & .00375 \\ 215 . & 72.7245 & .09736 & 127.52 & .00374 \\ 220 . & 72.1919 & .09595 & 122.06 & .00372 \\ 225 . & 71.6609 & .09455 & 116.99 & .00370 \\ 230 . & 71.1316 & .09315 & 112.28 & .00368 \\ 235 . & 70.6038 & .09176 & 107.90 & .00366 \\ 240 . & 70.0777 & .09038 & 103.81 & .00364 \\ 245 . & 69.5532 & .08901 & 100.00 & .00362\end{array}$

230. 69.0304

255. 68.5092

260.67 .9898

.08765

.08631

.08497
.08366

$\begin{array}{ll}66.9559 & .08236 \\ 66.4416 & .08109\end{array}$

275. $66.4416 \quad .08109$

$\begin{array}{lll}280 . & 65.9291 & .07983 \\ 285 . & 65.4184 & .07859\end{array}$

290. $64.9095 \quad .07738$

$96.44 \quad .00360$

$93.10 \quad .00358$

$\begin{array}{ll}89.98 & .00356 \\ 87.04 & .00354\end{array}$

$84.29 \quad .00352$

$81.70 \quad .00350$

$79.26 \quad .00348$

$76.96 \quad .00345$

$72.75 \quad .00341$

300. $63.6977 \quad .07501$

310.

310.62 .8939

$320 . \quad 61.8988$

$330 . \quad 60.912$

$350 . \quad 59.9368$

350.
360. $\quad 58.9713$

$\begin{array}{ll}360 . & 58.0170 \\ 370 . & 57.0748\end{array}$

$380 . \quad 56.1452$

55.2292

.07275
.07058

.06851

.06851

.06468

.06291

.06124

.05966

$70.81 \quad .00339$

$67.24 \quad .00336$

$64.03 \quad .00332$

$61.14 \quad .00329$

.00326

$\begin{array}{ll}56.16 & .00323 \\ 54.00 & .00321\end{array}$

$\begin{array}{ll}56.00 & .00321 \\ 52.04 & .00319\end{array}$

$50.24 \quad .00317$

400. $\quad 54.3273 \quad .05677$

$410 . \quad 53.4402 \quad .05544$

420. $\quad 52.5684 \quad .05419$

430. $\quad 51.7126 \quad .05301$

$440 . \quad 50.8731 \quad .05190$

50.0503

4.

$470 . \quad 48.456$

$\begin{array}{ll}480 . & 47.6851 \\ 490 . & 46.9315\end{array}$

.04986

.04986
.04892

.04803

.04719

.00316

$47.10 \quad .00315$

$44.45 \quad .00314$

$43.28 \quad .00314$

$42.20 \quad .00314$

$42.21 \quad .00314$

$41.21 \quad .00314$

30.290 .00316

$39.45 \quad .00316$

$37.94 \quad .00338$

$35.4 B \quad .00313$

500. 46.1955

.04639

$37.26 \quad .00320$

$34.35 \quad 000315$

$\begin{array}{lll}.04196 & 33.84 & .00320 \\ .04136 & 33.37 & .00323\end{array}$

510.
320. 45.4770

330. $\quad 44.0919$

.04563

$36.64 \quad .00321$

$35.33 \quad .00325$

- Two phase Boundary

.04357

.00327 
Table 7. Transport Properties of nxygen, Isobars, Engr. Units.

12000. psia Isobar

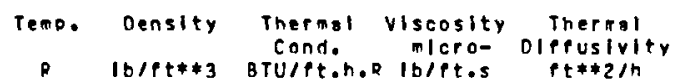

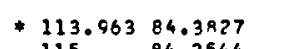

120 . 83.6482

125. 83.0581

$130 . \quad 82.4801$

135. 81.911

$\begin{array}{ll}140 . & 81.3504 \\ 145 . & 80.7953\end{array}$

150. $\quad 80.2451$

$\begin{array}{ll}1550.07906 \\ 160 . & 79.1566 \\ 1650 & 78.5172\end{array}$

155. 78.5172

175. 77.5464

$175 . \quad 77.5464$

$187 . \quad 77.0146$

$\begin{array}{ll}185 . & 76.4749 \\ 190 . & 75.9573\end{array}$

195. 75.4317

200. $\quad 74.9080$

$\begin{array}{ll}205 . & 74.3562 \\ 210 . & 73.8663\end{array}$

$\begin{array}{ll}215 . & 73.3483 \\ 220 . & 72.8321\end{array}$

225.72 .3177

230.71 .8052

235. 71.2946

$240 . \quad 70.785$

$\begin{array}{ll}250 . & 69.7739 \\ 255 & 69.2707 \\ 260 . & 68.7695\end{array}$

$\begin{array}{ll}260 . & 68.7695 \\ 265 . & 68.2702\end{array}$

$\begin{array}{ll}265 . & 68.2702 \\ 270 . & 67.7728\end{array}$

$\begin{array}{ll}270 . & 67.7728 \\ 275 . & 67.2774\end{array}$

280.66 .7839

$\begin{array}{ll}2850 & 66.2926 \\ 290 . & \quad 5.8032\end{array}$

$295 . \quad 65.3160$

300. 64.8308

$\begin{array}{ll}300 . & 64.8308 \\ 3100 & 63.8672 \\ 320 . & 62.9126\end{array}$

$330 . \quad 61.9675$

$340 . \quad 61.0324$

350. $\quad 60.1078$

$\begin{array}{ll}360 . & 59.1942 \\ 370 . & 58.2922\end{array}$

$\begin{array}{ll}370 . & 58.2922 \\ 380 . & 57.4023\end{array}$

390 . 56.5252

400. $\quad 55.6612$

410 . $\quad 54.8109$

$420 . \quad 53.9747$

430.53 .1531

440.52 .3463

$\begin{array}{ll}450 . & 51.5548 \\ 460 . & 50.7786\end{array}$

$\begin{array}{ll}460 . & 50.7786 \\ 470 . & 50.018\end{array}$

$\begin{array}{ll}480 . & 49.2732 \\ 490 . & 48.5442\end{array}$

$5000 \quad 47.8310$

$\begin{array}{ll}5000 & 47.8310 \\ 5100 & 47.1336\end{array}$

520.46 .4519

530.45 .7857

45.1351
.12909

.12866
.12670

.12670

.12324

.12167

.12016

.11731

.11456

.11320

.11050

.11050

. 10730

.10730

.10508

.10372

.10235
.10098

.10098
.09961

.09961

.09687

.09550

.09414

.09279

.09011

.08878

.08748

.08491

- 08365

.08241

- 08119

.07999
.07881

.07765

.07541

.07326

.06924

.06738

.06738

.06361

.06394

.06084

.05942

.05072

.05680

.05559
.05445

.05336

.05233

.05135

.05042

.04870

.04870
.04789

.04713

.04639
.04569
$462.83 \quad .00387$

$\begin{array}{ll}.55 .02 & .00387\end{array}$

$\begin{array}{ll}419.76 & .00389\end{array}$

$359.53 \quad .00390$

$333.77 \quad .00391$

$\begin{array}{ll}310.49 & .00391 \\ 289.43 & .00392\end{array}$

$270.35 \quad .00392$

253.174

237.32

223.00

209.96

.00390

168.04

$159.64 \quad .00388$

$151.90 \quad .00385$

$144.77 \quad .00384$

$138.18 \quad .00382$

$126.44 \quad .00379$

$121.21 \quad .00378$

$116.34 \quad .00376$

$111.81 \quad .00374$

$107.60 \quad .00372$

$99.98 \quad .00369$

$96.54 \quad .00367$

$93.31 \quad .00365$

$90.29 \quad .00363$

$87.45 \quad .00361$

$84.78 \quad .00359$

$82.26 \quad .00357$

$79.89 \quad .00355$

$\begin{array}{ll}77.66 & .00353 \\ 75.55 & .00351\end{array}$

$73.55 \quad .00349$

$69.88 \quad .00346$

$66.58 \quad .00342$

.00336

$58.47 \quad .00336$

$50.47 \quad .00334$

$56.25 \quad .00331$

$\begin{array}{ll}54.22 & .00329 \\ 52.38 & .00328\end{array}$

$30.69 \quad .00326$

$49.13 \quad .00325$

$47.71 \quad .00324$

$46.40 \quad .00324$

$\begin{array}{ll}45.19 & .00323 \\ 44.08 & .00323\end{array}$

$44.08 \quad .00323$

$43.05 \quad .00323$

$41.21 \quad .00324$

$40.40 \quad .00325$

38.94

38.29

37.68

37.12
36.59

.00327

.00328

.0032

.00330
$132.09 \quad .00383$

13000. DsI: Isobar

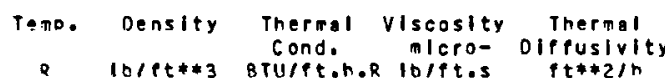

* Two Phese Boundary 
Table 7. Transoort Properties of Dxyoen. Isobars, Engr. Units.

14000. psta isobar

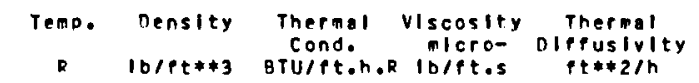

15000 . os 1. Isober

Temp. Density Thermal Viscosity Thermal

R Ib/ft**3 BTu/ftiog ib/ftos olfusivity

\begin{tabular}{|c|c|c|c|c|c|c|c|c|c|}
\hline $\begin{array}{l}116.480 \\
120 . \\
125 . \\
130 . \\
135 . \\
140 . \\
145 .\end{array}$ & $\begin{array}{l}84.7737 \\
84.3548 \\
83.7806 \\
83.2188 \\
82.6894 \\
82.1269 \\
81.5927\end{array}$ & $\begin{array}{l}.13095 \\
.12962 \\
.12797 \\
.12626 \\
.12476 \\
.12333 \\
.12195\end{array}$ & $\begin{array}{l}478.56 \\
451.58 \\
416.78 \\
385.61 \\
357.61 \\
332.39 \\
309.64\end{array}$ & $\begin{array}{l}.00390 \\
.00392 \\
.00394 \\
.00396 \\
.00397 \\
.00398 \\
.00399\end{array}$ & $\begin{array}{l}\text { * } 117.723 \\
120 . \\
125 . \\
130 . \\
135 . \\
140 . \\
145 .\end{array}$ & $\begin{array}{l}84.9630 \\
84.6967 \\
84.1263 \\
83.5716 \\
83.0291 \\
82.4963 \\
81.9714\end{array}$ & $\begin{array}{l}.13188 \\
.13104 \\
.12931 \\
.12773 \\
.12625 \\
.12485 \\
.12351\end{array}$ & $\begin{array}{l}485.78 \\
467.73 \\
431.30 \\
398.76 \\
369.60 \\
343.38 \\
319.75\end{array}$ & $\begin{array}{l}.00392 \\
.00393 \\
.00396 \\
.00398 \\
.00400 \\
.00401 \\
.00402\end{array}$ \\
\hline $\begin{array}{l}150 . \\
1550 \\
160 . \\
165 \circ \\
170 . \\
175 \circ \\
180 . \\
1850 \\
190 . \\
195 .\end{array}$ & $\begin{array}{l}81.0644 \\
80.5412 \\
80.0222 \\
79.5070 \\
78.9951 \\
78.4862 \\
77.9801 \\
77.6766 \\
76.9755 \\
76.4768\end{array}$ & $\begin{array}{l}.12062 \\
.11932 \\
.11803 \\
.11676 \\
.11549 \\
.11422 \\
.11295 \\
.11167 \\
.11039 \\
.10011\end{array}$ & $\begin{array}{l}289.07 \\
270.45 \\
253.55 \\
238.19 \\
224.21 \\
211.46 \\
199.81 \\
139.15 \\
179.38 \\
170.40\end{array}$ & $\begin{array}{l}.00400 \\
.00400 \\
.00400 \\
.00400 \\
.00400 \\
.00400 \\
.00400 \\
.00399 \\
.00399 \\
.00398\end{array}$ & $\begin{array}{l}150 . \\
1550^{\circ} \\
160 . \\
1650^{\circ} \\
170 . \\
1750^{\circ} \\
180^{\circ} \\
1850^{\circ} \\
190 . \\
195 .\end{array}$ & $\begin{array}{l}81.4529 \\
80.9398 \\
80.4314 \\
79.9270 \\
79.4262 \\
78.9287 \\
78.4341 \\
77.9423 \\
77.4531 \\
75.9663\end{array}$ & $\begin{array}{l}.12222 \\
.12095 \\
.11971 \\
.11847 \\
.11724 \\
.11601 \\
.11477 \\
.11353 \\
.11229 \\
.11104\end{array}$ & $\begin{array}{l}298.42 \\
279.12 \\
261.63 \\
245.74 \\
231.28 \\
218.10 \\
206.07 \\
195.06 \\
184.97 \\
175.70\end{array}$ & $\begin{array}{l}.00403 \\
.00404 \\
.00404 \\
.00405 \\
.00405 \\
.00405 \\
.00405 \\
.00405 \\
.00404 \\
.00404\end{array}$ \\
\hline $\begin{array}{l}200 . \\
205 \\
210 \\
2150 \\
220 \\
2250 \\
230 \\
2350 \\
240 . \\
245 .\end{array}$ & $\begin{array}{l}75.9804 \\
75.4863 \\
74.9943 \\
74.5045 \\
74.0168 \\
73.5313 \\
73.0479 \\
72.5667 \\
72.0876 \\
71.0107\end{array}$ & $\begin{array}{l}10791 \\
.10651 \\
.10321 \\
.10390 \\
.10259 \\
.10128 \\
.05997 \\
.09866 \\
.09735 \\
.09606\end{array}$ & $\begin{array}{l}162.14 \\
154.53 \\
147.51 \\
141.01 \\
135.00 \\
129.42 \\
124.24 \\
119.42 \\
114.93 \\
110.74\end{array}$ & $\begin{array}{l}.00397 \\
.00396 \\
.00395 \\
.00394 \\
.00393 \\
.00392 \\
.00390 \\
.00389 \\
.00387 \\
.00386\end{array}$ & $\begin{array}{l}200 . \\
205 . \\
210 . \\
215 . \\
220 . \\
2250 \\
230 . \\
2350 \\
240 . \\
2+5 .\end{array}$ & $\begin{array}{l}76.4820 \\
76.0001 \\
75.5205 \\
75.0431 \\
74.5680 \\
74.0951 \\
73.6245 \\
73.1561 \\
72.6899 \\
72.2260\end{array}$ & $\begin{array}{l}.10977 \\
.10851 \\
.10723 \\
.10595 \\
.10467 \\
.10339 \\
.10210 \\
.10082 \\
.09953 \\
.09826\end{array}$ & $\begin{array}{l}167.18 \\
159.33 \\
152.08 \\
145.39 \\
139.18 \\
133.43 \\
128.10 \\
123.13 \\
118.50 \\
114.19\end{array}$ & $\begin{array}{l}.00403 \\
.00402 \\
.00401 \\
.00400 \\
.00399 \\
.00398 \\
.00397 \\
.00396 \\
.00394 \\
.00393\end{array}$ \\
\hline $\begin{array}{l}250 . \\
2550 \\
2600 \\
2650 \\
270 \\
2750 \\
280 . \\
2850 \\
290 \\
295\end{array}$ & $\begin{array}{l}71.1359 \\
70.6633 \\
70.1929 \\
69.7247 \\
69.2586 \\
68.7948 \\
68.3333 \\
67.8740 \\
67.4169 \\
66.9522\end{array}$ & $\begin{array}{l}.09477 \\
.09349 \\
.09222 \\
.09096 \\
.08972 \\
.08849 \\
.08728 \\
.08608 \\
.08491 \\
.08375\end{array}$ & $\begin{array}{r}106.83 \\
103.17 \\
99.75 \\
96.53 \\
93.52 \\
70.68 \\
88.01 \\
85.50 \\
83.13 \\
80.89\end{array}$ & $\begin{array}{l}.00384 \\
.00382 \\
.00381 \\
.00379 \\
.00377 \\
.00376 \\
.00374 \\
.00372 \\
.00370 \\
.00369\end{array}$ & $\begin{array}{l}250 . \\
255 \circ \\
260 . \\
265 \circ \\
270 . \\
275 \circ \\
280 . \\
2850 \\
290 \\
275\end{array}$ & $\begin{array}{l}71.7643 \\
71.3049 \\
70.8477 \\
70.3928 \\
69.9401 \\
69.4898 \\
69.0418 \\
68.5960 \\
68.1527 \\
67.7116\end{array}$ & $\begin{array}{l}.09699 \\
.09573 \\
.09447 \\
.09323 \\
.09201 \\
.09079 \\
.08959 \\
.08841 \\
.08725 \\
.08610\end{array}$ & $\begin{array}{r}110.16 \\
106.39 \\
102.86 \\
99.55 \\
96.45 \\
93.53 \\
90.78 \\
88.19 \\
85.75 \\
83.45\end{array}$ & $\begin{array}{l}.00391 \\
.00390 \\
.00388 \\
.00387 \\
.00385 \\
.00383 \\
.00382 \\
.00380 \\
.00378 \\
.00377\end{array}$ \\
\hline $\begin{array}{l}300 . \\
310 . \\
320 . \\
330 . \\
340 . \\
350 . \\
360 . \\
370 . \\
380 . \\
300 .\end{array}$ & $\begin{array}{l}66.5098 \\
65.6119 \\
64.7237 \\
63.8452 \\
62.9768 \\
62.1188 \\
61.2714 \\
60.4349 \\
59.6096 \\
58.7959\end{array}$ & $\begin{array}{l}.08262 \\
.08041 \\
.07828 \\
.07625 \\
.07430 \\
.07244 \\
.07067 \\
.06898 \\
.06737 \\
.06584\end{array}$ & $\begin{array}{l}78.77 \\
74.88 \\
71.38 \\
68.23 \\
65.38 \\
62.79 \\
60.44 \\
58.30 \\
56.35 \\
54.35\end{array}$ & $\begin{array}{l}.00367 \\
.00364 \\
.00361 \\
.00358 \\
.00355 \\
.00353 \\
.00350 \\
.00348 \\
.00346 \\
.00345\end{array}$ & $\begin{array}{l}300 . \\
310 . \\
320 . \\
330 . \\
340 . \\
350 . \\
360 . \\
370 . \\
380 . \\
390 .\end{array}$ & $\begin{array}{l}67.2729 \\
66.4027 \\
65.5422 \\
64.6915 \\
63.8508 \\
63.0203 \\
62.2003 \\
61.3910 \\
60.5925 \\
59.8051\end{array}$ & $\begin{array}{l}.08497 \\
.08277 \\
.08066 \\
.07863 \\
.07669 \\
.07483 \\
.07305 \\
.07136 \\
.06974 \\
.05820\end{array}$ & $\begin{array}{l}81.27 \\
77.27 \\
73.67 \\
70.43 \\
67.50 \\
54.84 \\
62.42 \\
60.22 \\
58.21 \\
56.36\end{array}$ & $\begin{array}{l}.00375 \\
.00372 \\
.00369 \\
.00366 \\
.00364 \\
.00361 \\
.00359 \\
.00357 \\
.00355 \\
.00353\end{array}$ \\
\hline $\begin{array}{l}400 . \\
4100 \\
420 . \\
430 . \\
440 . \\
450 . \\
460 . \\
470 . \\
480 . \\
490 .\end{array}$ & $\begin{array}{l}57.9930 \\
57.2041 \\
56.4267 \\
55.6618 \\
54.9098 \\
54.1707 \\
53.4447 \\
52.7319 \\
52.0324 \\
51.3462\end{array}$ & $\begin{array}{l}.06439 \\
.06300 \\
.06168 \\
.06042 \\
.05922 \\
.05807 \\
.05698 \\
.05593 \\
.05492 \\
.05396\end{array}$ & $\begin{array}{l}52.91 \\
51.40 \\
50.00 \\
48.72 \\
47.53 \\
46.43 \\
45.41 \\
44.47 \\
43.59 \\
42.78\end{array}$ & $\begin{array}{l}.00343 \\
.00342 \\
.00341 \\
.00341 \\
.00340 \\
.00340 \\
.00339 \\
.00339 \\
.00339 \\
.00339\end{array}$ & $\begin{array}{l}400 . \\
410 . \\
420 . \\
430 . \\
440 . \\
450 . \\
460 . \\
470 . \\
480 . \\
490 .\end{array}$ & $\begin{array}{l}59.0290 \\
58.2645 \\
57.5116 \\
56.7707 \\
56.0417 \\
55.3249 \\
54.6204 \\
53.9281 \\
53.2483 \\
52.5808\end{array}$ & $\begin{array}{l}.06673 \\
.06532 \\
.06398 \\
.06270 \\
.06147 \\
.06030 \\
.05917 \\
.05809 \\
.05705 \\
.05604\end{array}$ & $\begin{array}{l}54.67 \\
53.12 \\
51.68 \\
50.36 \\
49.14 \\
48.00 \\
46.95 \\
45.98 \\
45.07 \\
44.23\end{array}$ & $\begin{array}{l}.00352 \\
.00331 \\
.00350 \\
.00349 \\
.00348 \\
.00347 \\
.00347 \\
.00346 \\
.00346 \\
.00346\end{array}$ \\
\hline $\begin{array}{l}500 . \\
510 . \\
520 . \\
530 . \\
540 .\end{array}$ & $\begin{array}{l}50.6733 \\
50.0137 \\
49.3674 \\
48.7342 \\
48.1140\end{array}$ & $\begin{array}{l}.05303 \\
.05214 \\
.05139 \\
.05046 \\
.04957\end{array}$ & $\begin{array}{l}42.02 \\
41.31 \\
40.65 \\
40.04 \\
39.47\end{array}$ & $\begin{array}{l}.00340 \\
.00340 \\
.00340 \\
.00341 \\
.00341\end{array}$ & $\begin{array}{l}500 . \\
510 . \\
520 . \\
530 . \\
540 .\end{array}$ & $\begin{array}{l}51.9257 \\
51.2830 \\
50.6526 \\
50.0344 \\
49.4283\end{array}$ & $\begin{array}{l}.05508 \\
.05415 \\
.05325 \\
.05239 \\
.05155\end{array}$ & $\begin{array}{l}43.45 \\
42.71 \\
42.03 \\
41.40 \\
40.80\end{array}$ & $\begin{array}{l}.00346 \\
.00346 \\
.00346 \\
.00346 \\
.00346\end{array}$ \\
\hline
\end{tabular}


The IIstings ore extracted from reference [ 7$]$.

Note I. A call to the coefficlent surroutine, l.e. CALL DATA 02, must precede the use of these routlnes. Note 2. Entry varlables to FUNCTION VISC $(D D, T)$ are denslty in molll ond temoerature in $K$.

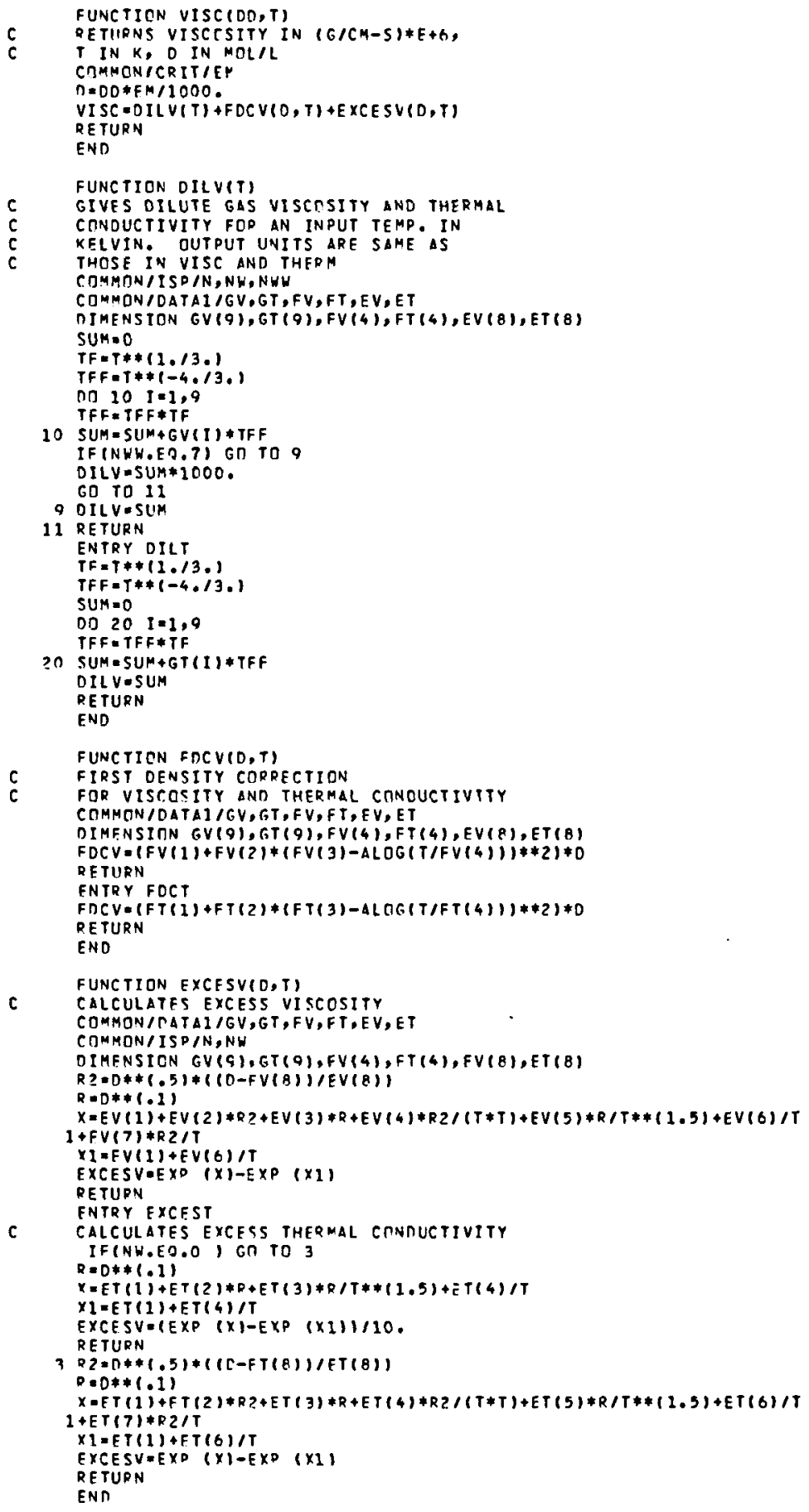


The listings ore extracted from references [7] and [8].

Note 1. A call to the coefflelent subroutine, l.e. CALL OATA 02, must precede the use of these routines. Note 2. Entry varlables to FUNCTION THERM(DD,T) are density in molll and temperature in $K$.

Note 3. The dilute gas thermal conductivity, ENTRY DILT In FUNCTION DILV(T), is ilsted in Appendix A.

FUNCTION THERMIOD,TI

C RETURNS TC IN MWIM-K, T IN K, D IN MOL/L

COMMON IHAN/CR, TCI

COMMON /ISP/N,NH

COMMON/CRITIEM

$\rightarrow \quad$ IF(EM.EO.31.9988) GOTO 4

$D=D D * E M / 1000$.

IF (NH.EO.O) GO TO 3

$C R=0.0$

$T H E R=D I L T(T)+F D C T(D, T) * 100,+E X C E S T(0, T)+C R$

TCI $=$ THER-CR

THERM = THER

RETURN

$3 C R=0.0$

THERM=OILT $(T)+F D C T(D, T)+E X C E S T(D, T)+C R$

TCI $=$ THERM-CR

RETURN

$\rightarrow 4$ CR-CRITCR (DD,T)*1000.

$\rightarrow$ TCI=THERMR $(D D, T) \neq 1000$.

$\rightarrow \quad T H E R M=T C I+C R$

$\rightarrow$ RETURN

END

FUNCTION THERMR(RHO, TEMP)

C 4 TH SURFACE, COEF. FROM TCO21 AND MINIMS, 3 MAR 82 DIMENSION B(10)

DATA B/.298644E-5

$1, .59842 E+00, .11362 E-01,-.19520 E-04$

$2, .47624 E+00,-.64769 E-03, .83223 E-06$

$3,-.278141 E-4, .153705 E-6, .147176 E+1 /$

$T=$ TEMP

DEN $=$ RHO

TCZERO-DILTITI/1000.

$A L=B(1) * T$

$B E=B(2)+B(3) * T+B(4) * T * * 2$

$G A=B(5)+B(6) * T+B(7) * T * * 2$

$D E=B(8)+B(9) * T+B(10) / T * * 2$

THERMR $=T C Z E R C+A L * D E N+D E *(E X P(B E * D E N * * G A)-1.0)$

RETURN

END

FUNCTION CRITCR(RHO, TEMP)

C 4 TH SURFACE, COEF. FROM TCO2I AND MINIMS, 3 MAR BZ

DIMENSION C $(7)$

DATA C 1.2192 COE+0,-145.55,.734512E-02,-.282950E-04

$1,0.71599 E-3,013804 E+0, .12980 E-31$

DATA (TC $=154.581),($ RHOC $=13.63)$

T:TEKP

DEN $=$ RHO

$D E L D=A B S(D E N-R H O C) / R H D C$

IF (T.LT.TC) $T=T C+(T C-T)$

IF (T.LT.307.443) GO TO 4

CR ITCR $=0$.

RETURN

4 CONTINUE

$\triangle M P L=C(1) /(T+C(2))+C(3)+C(4) * T$

DELT-T-TC

RHOCENT $=$ RHOC +C (5) *DELT $* 1.5$

$D E L R H D=D E N-R+O C E N T$

$X 1=C(6) * D E L R H D$

IF (DELRHO.LT.0.) $\times 1=\times 1+C(7) * D E L R H O * * 5$

CRITCR=AM $P L * E X P(-X 1 * * 2)$

IF (T.GT.162.9805) RETURN

IF(DEN.LT.7,5.OR.OEN.GT.18.) RETURN

TESTI-SENGBIIDEN,TI

IF(TEST1.GT.CRITCR) CRITCR -TESTI

RETURN

END 
FUNCTION SENGBI (RHD, TEMP)

C SCALED EOUATION ONLY, VERSION OF 12 FEB 82

C CRITICAL ENHANCEMENT AS IN SENGERS ET AL 1981 U MARYL. REPORT

UNITS, IN MOL/L,K, INTERNAL ALSD ATM, OUT W/M-K, ETA G/CM-S,BK J/K

1.02 REPLACED BY 1.04, PARAMETER VARIATION FOR WEBER OATA

DATA $(T C=154.581),(D C=13.63),(B K=1.38054 E-23),(P C=49.77054)$

$1,(Z Z=5.9783 E-10)$

DATA $(E=0.287),(G=1.190),(B=0.355),(D D=2.36),(X Z=0.183),(D E=4.352)$

$D E N=R H O$

$T=T E M P$

$D E L D=A B S(D E N-D C) / D C$

DELT $\because A B S(T-T C) / T C$

$O F A C T=E X P(-(39.8 * D E L T * 2+5.45 * D E L D * 4))$

RSTAR=DEN/DC

VIS =VISC (DEN,T)*(1,OE-06)

CALL DPOT (DPT, OEN,T)

C IF(DELD.LE.0.25.AND.DELT.LT.0.03) GO TO 8

C CALL DPDD (DPD,DEN,T)

C CHISTAR $=P C * D E N /(D C * \# 2 * D P D)$

GO TO 12

B IF(DELD.EO.0.) GO TO 3

$X=D E L T / D E L D * *(1.0 / B)$

$Y=(X+X Z) / X Z$

$T O P=D E(D * *(-G / B) *((1,+E) /(1,+E * Y * *(2, * B))) * *((G-1) /,(2, * B))$

$D I V=D D *(D E+(Y-1) *,(D E-1, / B+E * Y * *(2, * B)) /(1,+E * Y * *(2, * B)))$ CHISTAR = TOP /DIV

$12 C H I=C H I S T A R * * 0.468067$

UPPER $=1.04 * 8 K / P C *(T * D P T / R S T A R) * 2 * C H T * 0 F A C T * 1.01325 E * 6$

SENGB1 =UPPER $/(2 Z * 6 . * 3.14159 * V I S)$

RETURN

$3 B G A M=X Z * * G / D O *(1,1 .+E) / E) * *(1 G-1,1 /(2 * B))$

$C H I S T A R=B G A M *(D E L T) * *(-G)$

60 TO 12

END 
Temperature

Pressure

Specific Volume

Internal Energy, Enthalpy

Entropy, Specific Heat

Thermal Conductivity

Viscosity

Speed of Sound

Molecular Weight

Surface Tension
$1.8 \mathrm{R}=1 \mathrm{~K}$

$14.695949 \mathrm{psia}=1 \mathrm{~atm}=1.01325 \times 10^{5} \mathrm{~N} / \mathrm{m}^{2}$

$$
\left(1 \mathrm{~N} / \mathrm{m}^{2}=1 \mathrm{~Pa}\right)
$$

$0.0005005957 \mathrm{ft}^{3} / 1 \mathrm{~b}_{\mathrm{m}}=1 \mathrm{~cm}^{3} / \mathrm{g} \mathrm{mol}$

$0.0134446 \mathrm{BTU} / 1 \mathrm{~b}_{\mathrm{m}}=1 \mathrm{~J} / \mathrm{g} \mathrm{mol}$

$0.0074692 \mathrm{BTU} / 1 \mathrm{~b}_{\mathrm{m}} \mathrm{R}=1 \mathrm{~J} / \mathrm{g} \mathrm{mol}-\mathrm{K}$

$0.0578176 \mathrm{BTU} / \mathrm{ft}-\mathrm{hr}-\mathrm{R}=1 \mathrm{~mW} / \mathrm{cm}-\mathrm{K}$

$0.0671968971 \mathrm{~b}_{\mathrm{m}} / \mathrm{ft}-\mathrm{s}=1 \mathrm{~g} / \mathrm{cm}-\mathrm{s}=1 \mathrm{~N} \mathrm{~s} / \mathrm{m}^{2}$

$=1 \mathrm{~Pa} \mathrm{~s}$

$3.2808 \mathrm{ft} / \mathrm{s}=1 \mathrm{~m} / \mathrm{s}$

31.9988

$$
\begin{aligned}
0.5710147 \times 10^{-5} 1 \mathrm{~b}_{\mathrm{f}} / \mathrm{in} & =1 \mathrm{dyn} / \mathrm{cm} \\
(1 \mathrm{dyn} & \left.=10^{-5} \mathrm{~N}\right)
\end{aligned}
$$




\begin{tabular}{|c|c|c|}
\hline $\begin{array}{l}\text { 1. Report No. } \\
\text { NASA RP-1102 }\end{array}$ & 2. Government Accession No. & 3. Recipient's Catalog No. \\
\hline \multirow{2}{*}{\multicolumn{2}{|c|}{$\begin{array}{l}\text { 4. Title and Subtitle } \\
\text { TRANSPORT PROPERTIES OF OXYGEN }\end{array}$}} & $\begin{array}{ll}\text { 5. } & \text { Report Date } \\
\text { April } & 1983\end{array}$ \\
\hline & & 6. Performina Orqanization Code \\
\hline \multirow{2}{*}{\multicolumn{2}{|c|}{$\begin{array}{l}\text { 7. Author(s) } \\
\text { H. M. Roder }\end{array}$}} & $\begin{array}{l}\text { 8. Performing Organization Report No. } \\
\text { NBSIR } 82-1672\end{array}$ \\
\hline & & 10. Work Unit No. \\
\hline \multirow{2}{*}{\multicolumn{2}{|c|}{$\begin{array}{l}\text { 9. Performing Organization Name and Address } \\
\text { National Bureau of Standards } \\
\text { Department of Commerce } \\
\text { Washington, D. C. } 20234\end{array}$}} & $\begin{array}{l}\text { 11. Contract or Grant No. } \\
\mathrm{C}-32369-\mathrm{C}\end{array}$ \\
\hline & & \multirow{2}{*}{$\begin{array}{l}\text { 13. Type of Report and Period Covered } \\
\text { Ref erence Publication }\end{array}$} \\
\hline \multirow{2}{*}{\multicolumn{2}{|c|}{$\begin{array}{l}\text { 12. Sponsoring Agency Name and Address } \\
\text { National Aeronautics and Space Administration } \\
\text { Lewis Research Center } \\
\text { Cleveland, Ohio } 44135\end{array}$}} & \\
\hline & & 14. Sponsoring Agency Code \\
\hline
\end{tabular}

15. Supplementary Notes

16. Abstract

This report presents tables of viscosity, thermal conductivity, and thermal diffusivity of oxygen as a function of temperature and pressure from the triple point to $320 \mathrm{~K}$ and at pressures to $100 \mathrm{MPa}$. Auxiliary tables in engineering units are also given. Viscosity and thermal conductivity are calculated from previously published correlations. Density and specific heat at constant pressure, required to calculate thermal diffusivity, are obtained from an equation of state. The Prandtl number can be obtained quite easily from the values tabulated.

17. Key Words (Suggested by Author(s))

Density; Oxygen; Pressure; Tables;

Temperature; Thermal conductivity; Thermal diffusivity; Viscosity

18. Distribution Statement

STAR Category 34

Unclassified - unlimited
19. Security Classif, (of this report)

Unclassified
20. Security Classif. (of this page)

Unclassified
21. No, of Pages

87
22. Price* A05

* For sale by the National Technical Information Service, Springfield, Virginia 22161 

National Aeronautics and Space Administration

Washington, D.C.

20546

Official Business

Penalty for Private Use, $\$ 300$
Postage and Fees Paid National Aeronautics and Space Administration NASA-451

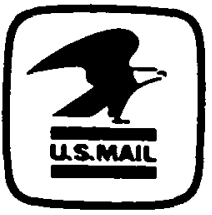

